\title{
Evolução dos Sistemas Hidrotermais na Extensão Sul da Bacia de Vazante: Implicações para Mineralização de Zinco Silicatado
}

ORIENTADORA: GEMA RIBEIRO OLIVO COORIENTADORA: MÁRCIA ABRAHÃO MOURA 
Ficha catalográfica

Universidade de Brasília

Biblioteca do Instituto de Geociências

CARVALHO, IGOR ABU KAMEL DE, 1989-

Evolução do Sistema Hidrotermal na Extensão Sul da Bacia de Vazante: Implicações para Mineralização de Zinco Silicatado / Igor Abu Kamel de Carvalho - Brasília, DF, 2015.

Orientador: Gema Ribeiro Olivo

Coorientador: Márcia Abrahão Moura

Dissertação de mestrado

Data de defesa: 17 de julho de 2015

Palavras chave: alteração hidrotermal, zinco silicatado, willemita, Grupo Vazante

\section{$\underline{\text { Informações para Biblioteca Digital }}$}

Título em outro idioma: Evolution of the Hydrothermal Systems in the Southern Extension of the Vazante Group: Implications to Silicate Zinc Mineralization

Palavras-chave em inglês: Hydrothermal alteration, zinc silicate, willemite, Vazante Group.

Área de concentração: Mineralogia e Petrologia

Titulação: Mestre em Geologia

Banca examinadora:

Gema Ribeiro Olivo (Orientadora e Presidente da banca examinadora)

Lena Virgínia Soares Monteiro

Carlos José Souza de Alvarenga

Claudio de Morisson Valeriano (suplente)

Bernhard Manfred Buhn (suplente)

Programa de Pós-graduação: Geologia 


\section{AGRADECIMENTOS}

Agradeço primeiramente à Votorantim Metais S.A. que permitiu acesso a dados e acesso ao testemunho de sondagem VZVG003 para este estudo. Agradeço fortemente à equipe da Votorantim pelo apoio na descrição do testemunho de sondagem e durante o trabalho de campo, representada por Basílio Botura Neto, Fernando Henrique Baia e, em especial, Gustavo Diniz Oliveira, pelas elucidativas discussões e apoio indispensável. Também agradeço ao Conselho Nacional de Desenvolvimento Científico e Tecnológico (CNPq) pela bolsa de mestrado.

Agradeço à Universidade de Brasília pelo apoio de infraestrutura laboratorial e de trabalho de campo. Agradeço ao Raimundo e Francisca da Laminação, Mancini e Eduardo do laboratório de isótopos estáveis. À professora Lucieth Vieira pelas discussões e à Jaqueline, Iris e Federico do laboratório de microssonda. Agradeço, também, o NSERC (Natural Sciences and Engineering Research Council), do Canadá, pelo apoio a Gema Olivo para a realização dessa pesquisa.

Agradeço fortemente à minha orientadora Gema Ribeiro Olivo (Queen's UniversityCanadá), pelas horas disponibilizadas para discussões, correções, incentivo e paciência, claramente indispensáveis para a realização desse trabalho. Também reforço meus agradecimentos à minha coorientadora Márcia Abrahão Moura, pela ajuda indispensável, incentivo, alertas e amizade. Vocês duas são parte integrante da minha memória profissional e pessoal. Muito obrigado.

Agradeço às pessoas que não podem mais me ouvir, como meu vô Dadinho, mas que determinantemente me influenciaram a seguir este caminho. Fiquem em paz. Agradeço e dedico este trabalho a meus pais, Rogério Teixeira de Carvalho e Ivete Chafik Abu Kamel.

Obrigado a todos,

Igor Abu Kamel de Carvalho 


\section{RESUMO}

Durante a evolução da Extensão Sul do Grupo Vazante da Província Tocantins, Minas Gerais, Brasil, múltiplos estágios de interação fluido-rocha foram reconhecidos. As alterações hidrotermais relacionadas a esses múltiplos estágios foram documentadas em rochas carbonáticas dos membros Morro do Pinheiro Superior e Pamplona Inferior, da Formação Serra do Poço Verde. Essas unidades são as principais hospedeiras dos depósitos hipogênicos, estruturalmente controlados, de zinco silicatado do distrito de Vazante, incluindo a mina de Vazante, considerada o maior depósito willemítico $\left(\mathrm{Zn}_{2} \mathrm{SiO}_{4}\right)$ do mundo, com recursos totais estimados de 40 a $60 \mathrm{Mt}$ a $20 \%$ de $\mathrm{Zn}$. Seis estilos de alteração hidrotermal foram identificados na Extensão Sul do Grupo Vazante. (I) Estágio de alteração precoce que compreende bandas de substituição de dolomita sem enriquecimento apreciável de metais, precipitadas a partir de fluidos de moderadas salinidades, compostos por $\mathrm{H}_{2} \mathrm{O}-\mathrm{NaCl}-\mathrm{CaCl}_{2}$, com temperaturas em torno de $90^{\circ} \mathrm{C}$, interpretados como sendo relacionados à tardi-diagênese. As composições isotópicas de $\mathrm{C}$ e $\mathrm{O}$ dos fluidos são os valores mais leves registrados (-3.62\%o a $-2.60 \%$ PDB e $+4.60 \%$ a $+5.95 \%$ o SMOW, respectivamente, a $90^{\circ} \mathrm{C}$ ). Isso sugere origem meteórica ou conata para o fluido assim como interação com carbono orgânico. (II) $\mathrm{O}$ estágio pré-mineralização é evidenciado por precipitação de dolomita dog-tooth, quartzo e traços de oxi/hidróxidos de $\mathrm{Fe}$ que preenchem cavidades de dissolução. Os fluidos associados a essa alteração são resultantes de mistura de fluidos de composição $\mathrm{H}_{2} \mathrm{O}-\mathrm{NaCl}-\mathrm{CaCl}_{2}$ de altas salinidades e fluidos de composição $\mathrm{H}_{2} \mathrm{O}-\mathrm{NaCl}-\mathrm{MgCl}_{2}$, de salinidades baixas a moderadas, e temperaturas em torno de $100^{\circ}$ a $150^{\circ}$ C. Suas composições isotópicas de C sugerem interação com carbono orgânico ($1.81 \%$ a $-1.04 \%$ o $\mathrm{PDB}$ a $120^{\circ} \mathrm{C}$ ). (III) $\mathrm{O}$ estágio mineralizante engloba quatro fases de precipitação mineral. A primeira é distal e caracterizada por dolomitos avermelhados devido a hematita e dolomita vermelha disseminada e por conteúdos mais elevados de $\mathrm{Al}_{2} \mathrm{O}_{3}, \mathrm{P}_{2} \mathrm{O}_{5}$, e alguns elementos de terras raras (ETR's: Ce, Dy, Er, Eu, Gd, Nd, Pr, Sm, Tb) e Ni. A segunda fase é o estágio principal de mineralização, constituído por dolomita vermelha e hematita maciças e willemita, com valores mais elevados em $\mathrm{SiO}_{2}, \mathrm{Fe}_{2} \mathrm{O}_{3}, \mathrm{P}_{2} \mathrm{O}_{5}, \mathrm{Mn}, \mathrm{As}, \mathrm{Ge}, \mathrm{Hg}$, In, U, $\mathrm{V}, \mathrm{Ag}, \mathrm{Cd}, \mathrm{Cu}, \mathrm{Mo}, \mathrm{Ni}, \mathrm{Pb}, \mathrm{Sb}$, Se e $\mathrm{Zn}$. A terceira fase compreende dolomita branca, hematita e traços de willemita, com valores mais elevados em $\mathrm{Mn}, \mathrm{Ba}, \mathrm{Cr}, \mathrm{Cd}, \mathrm{Ni}, \mathrm{Pb}$ e As. As composições isotópicas de $\mathrm{C}$ e $\mathrm{O}$ para os fluidos em equilíbrio com dolomita para essas duas fases são similares e são tamponadas pela composição isotópica dos dolomitos hospedeiros $\left(\delta^{13} \mathrm{C}=+0,78 \%\right.$ a $+2,88 \%$ PDB e $\delta^{18} \mathrm{O}=+23,79 \%$ a $+29,74 \%$, a $\left.180^{\circ} \mathrm{C}\right)$ onde essas fases são incipientes $\left(\delta^{13} \mathrm{C}=+1,23 \%\right.$ a $+1,61 \%$ o PDB e $\delta^{18} \mathrm{O}=+26,22 \%$ a $+27,55 \%$ a $\left.180^{\circ} \mathrm{C}\right)$. Onde essas três fases são mais pervasivas, a composição isotópica dos fluidos é mais leve $\left(\delta^{13} \mathrm{C}=\right.$ $1,62 \%$ a $+1,41 \%$ o PDB e $\delta^{18} \mathrm{O}=+16,46 \%$ a $+21,51 \%$ a $\left.180^{\circ} \mathrm{C}\right)$. A quarta fase corresponde a $\mathrm{Zn}$-clorita e quartzo precipitados a partir de fluidos misturados: um composto por $\mathrm{H}_{2} \mathrm{O}-\mathrm{NaCl}$, de salinidades moderadas a altas e outro de salinidades baixas a moderadas, com temperaturas em torno de $170^{\circ}$ a $190^{\circ} \mathrm{C}$ e $90^{\circ}$ a $130^{\circ}$, respectivamente. (IV) A alteração sulfetada precoce compreende pirita, esfalerita, dolomita branca, hematita fina e quartzo tardio, com valores mais elevados em $\mathrm{Na}_{2} \mathrm{O}, \mathrm{SiO}_{2}, \mathrm{Sr}, \mathrm{Ba}, \mathrm{Cu}, \mathrm{Ni}, \mathrm{As}$, $\mathrm{Re}$ e $\mathrm{S}$. As composições isotópicas de $\mathrm{C}$ e $\mathrm{O}$ desse estilo são similares às três fases de dolomita do estágio mineralizante, e exibe os mesmos padrões onde é incipiente $\left(\delta^{13} \mathrm{C}=+2,09 \%\right.$ a $+2,92 \%$ PDB e $\delta^{18} \mathrm{O}=+27,22 \%$ a $+30,02 \%$ a $180^{\circ}$ C) e onde é pervasiva $\left(\delta^{13} \mathrm{C}=+0,37 \%\right.$ o $+2,27 \%$ o PDB e $\delta^{18} \mathrm{O}=+19,12 \%$ o $+25,73 \%$ a $\left.180^{\circ} \mathrm{C}\right)$. Inclusões fluidas revelaram mistura de fluidos em quartzo tardio dessa alteração, que são similares aos fluidos mistos reconhecidos no quartzo tardio do estágio de mineralização willemítica. Esses dados sugerem relação cogenética entre esses dois estilos de alteração. (V) A alteração sulfetada tardia é caracterizada por esfalerita, galena, calcocita, greenockita, covelita e 
dolomita branca, que cortam dolomita vermelha e hematita maciças do estágio mineralizante principal. (VI) O estágio tardio é caracterizado por silicificação. Localmente, quartzo dessa alteração reage com esfalerita mais precoce para produzir willemita. A composição isotópica de O mais leve dos fluidos do estágio mineralizante em equilíbrio com dolomita cogenética a willemita em zonas de alteração pervasiva indica o envolvimento de água meteórica evoluída e salmouras metalíferas. A composição isotópica de $\mathrm{C}$ mais leve sugere interação com carbono orgânico e/ou atividade bacteriológica durante a deposição de minério. Dados de inclusões fluidas apontam mistura de fluidos durante as fases tardias associadas ao estágio de mineralização willemítica e à alteração de pirita e esfalerita (alteração sulfetada precoce).

Palavras-chave: alteração hidrotermal, zinco silicatado, willemita, Grupo Vazante 


\section{ABSTRACT}

Many stages of fluid-rock interaction were recognized during the evolution of the Southern Extension of the Vazante Group from the Tocantins Province, Minas Gerais, Brazil. The hydrothermal alterations related to these multiple stages were documented in carbonate rocks of the Upper Morro do Pinheiro and Lower Pamplona members from the Serra do Poço Verde Formation. These units are the main host for the hypogene, structurally-controlled zinc silicate deposits in the Vazante $\mathrm{Zn}$ District, including the Vazante mine, which is considered to be the largest willemitic $\left(\mathrm{Zn}_{2} \mathrm{SiO}_{4}\right)$ deposit in the world, with estimated total resources of 40 to $60 \mathrm{Mt}$ at $20 \%$ of $\mathrm{Zn}$. Six hydrothermal alteration styles were identified in Southern Extension of the Vazante Group. (I) Early stage alteration comprises dolomite substitution bands and nodules, with no appreciable metal enrichment, precipitated from moderate salinities $\mathrm{H}_{2} \mathrm{O}-\mathrm{NaCl}-\mathrm{CaCl}_{2}$ fluids, with temperatures around $90^{\circ} \mathrm{C}$, interpreted as late-diagenetic. The calculated $\mathrm{C}$ and $\mathrm{O}$ isotopic compositions of the fluids yielded the lightest values $(-3.62 \%$ to $-2.60 \%$ PDB and $+4.60 \%$ to $+5.95 \%$ sMOW, respectively), suggesting meteoric and/or connate origin and interaction with organic carbon. (II) Pre-ore stage alteration is evidenced by dog-tooth dolomite and quartz with minor Fe-oxi/hydroxides which fills dissolution voids. The fluids associated to this alteration resulted by mixture of $\mathrm{H}_{2} \mathrm{O}-\mathrm{NaCl}-\mathrm{CaCl}_{2}$ high salinities fluids with $\mathrm{H}_{2} \mathrm{O}-\mathrm{NaCl}$ $\mathrm{MgCl}_{2}$ low to moderate salinities fluids, at temperatures around $100^{\circ}$ to $150^{\circ} \mathrm{C}$. The $\mathrm{C}$ isotopic also suggests interaction with organic carbon (-1.81\%o to -1.04\%). (III) Ore stage encompasses four phases of mineral precipitation. The first is distal and characterized by red stained dolostones due to disseminated hematite and red dolomite and by $\mathrm{Al}_{2} \mathrm{O}_{3}, \mathrm{P}_{2} \mathrm{O}_{5}$, and some rare earth elements (REE: Ce, Dy, Er, Eu, Ga, Gd, Nd, Pr, Sm, Tb) and Ni higher values. The second is the main phase of the ore stage, consisted of massive red dolomite, massive hematite and willemite with higher concentrations of $\mathrm{SiO}_{2}, \mathrm{Fe}_{2} \mathrm{O}_{3}, \mathrm{P}_{2} \mathrm{O}_{5}, \mathrm{Mn}, \mathrm{As}, \mathrm{Ge}, \mathrm{Hg}, \mathrm{In}, \mathrm{U}, \mathrm{V}, \mathrm{Ag}, \mathrm{Cd}$, $\mathrm{Cu}, \mathrm{Mo}, \mathrm{Ni}, \mathrm{Pb}, \mathrm{Sb}, \mathrm{Se}$, and $\mathrm{Zn}$. The third phase comprises white dolomite, hematite and traces of willemite with higher concentrations of $\mathrm{Mn}, \mathrm{Ba}, \mathrm{Cr}, \mathrm{Cd}, \mathrm{Ni}, \mathrm{Pb}$, and $\mathrm{As}$. The $\mathrm{C}$ and $\mathrm{O}$ isotopic compositions of the fluids in equilibrium with dolomite from these three phases are similar, and where they are incipient $\left(\delta^{13} \mathrm{C}=+1,23 \%\right.$ to $+1,61 \%$ PDB and $\delta^{18} \mathrm{O}=+26,22 \%$ to $+27,55 \%$ at $180^{\circ} \mathrm{C}$ ), their composition is buffered by the isotopic composition of the host dolostones $\left(\delta^{13} \mathrm{C}=+0,78 \%\right.$ o to $+2,88 \%$ o PDB and $\delta^{18} \mathrm{O}=+23,79 \%$ o to $+29,74 \%$, at $\left.180^{\circ} \mathrm{C}\right)$. However, they yielded lighter isotopic values where these alterations are more pervasive $\left(\delta^{13} \mathrm{C}=\right.$ $-1,62 \%$ to $+1,41 \%$ PDB and $\delta^{18} \mathrm{O}=+16,46 \%$ o to $+21,51 \%$ at $180^{\circ} \mathrm{C}$ ). The fourth phase corresponds to $\mathrm{Zn}$-chlorite and quartz precipitated from mixed fluids: One of $\mathrm{H}_{2} \mathrm{O}-\mathrm{NaCl}$ composition of moderate to high salinities and other of low to moderate salinities, with temperatures around $170^{\circ}$ to $190^{\circ} \mathrm{C}$ and $90^{\circ}$ to $130^{\circ} \mathrm{C}$, respectively. (IV) Early sulfide alteration composed of pyrite, sphalerite, white dolomite, fine hematite and late quartz, with higher concentrations of $\mathrm{Na}_{2} \mathrm{O}, \mathrm{SiO}_{2}, \mathrm{Sr}, \mathrm{Ba}, \mathrm{Cu}, \mathrm{Ni}, \mathrm{As}, \mathrm{Re}$, and $\mathrm{S}$. The $\mathrm{C}$ and $\mathrm{O}$ isotopic compositions of this style are similar to the three phases of dolomite of the ore stage, exhibiting the same patterns where it is incipient $\left(\delta^{13} \mathrm{C}=+2,09 \%\right.$ to $+2,92 \%$ PDB and $\delta^{18} \mathrm{O}=+27,22 \%$ to $+30,02 \%$ at $\left.180^{\circ} \mathrm{C}\right)$ or pervasive $\left(\delta^{13} \mathrm{C}=+0,37 \%\right.$ o to $+2,27 \%$ PDB and $\delta^{18} \mathrm{O}=+19,12 \%$ o to $+25,73 \%$ at $180^{\circ} \mathrm{C}$ ). Fluid inclusions revealed mixed fluids in late quartz from this alteration, which are similar to the fluids recognized in late quartz from the willemite ore stage. These data suggest co-genetic relationship of these two alteration styles. (V) The late sulfide alteration comprises sphalerite, galena, chalcocite, greenockite, covellite, and white dolomite, that crosscut the massive red dolomite and massive hematite from the main ore stage phase. (VI) The latest stage is characterized by silicification. Locally quartz from this alteration reacts with earlier sphalerite to produce willemite. The lighter $\mathrm{O}$ isotopic composition of the ore stage fluids 
in equilibrium with dolomite co-genetic to willemite in pervasive alteration zones indicates the involvement of evolved meteoric fluids and metalliferous brines. Lighter $\mathrm{C}$ isotopic composition implies interaction with organic carbon and/or bacteriological activity during ore deposition. Fluid inclusion data suggest that fluid mixing also took place during the late phases associated with the willemite ore stage and the pyrite-, sphalerite-bearing (early sulfide) alteration.

Key words: Hydrothermal alteration, zinc silicate, willemite, Vazante Group. 


\section{SUMÁRIO}

Capítulo 1

1

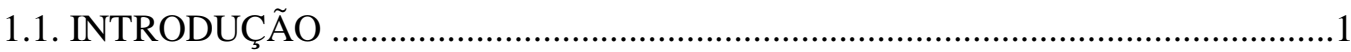

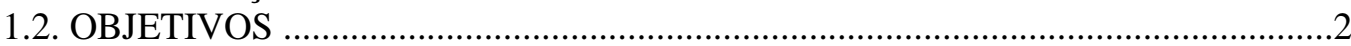

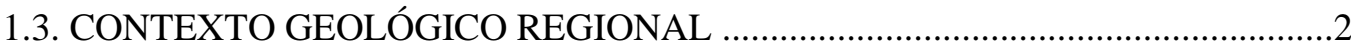

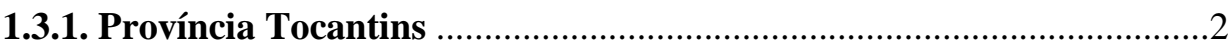

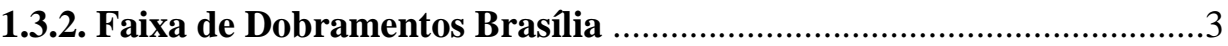

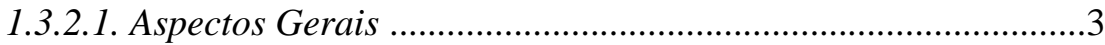

1.3.2.2. Configuração Tectônica da FDB .............................................4

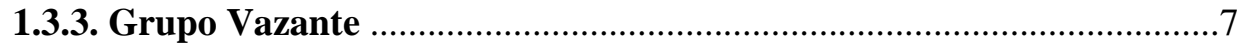

1.4. REVISAO DOS ESTUDOS DE INCLUSÕES FLUIDAS ASSOCIADAS A

DEPÓSITOS DE METAIS BÁSICOS EM BACIAS SEDIMENTARES ......................12

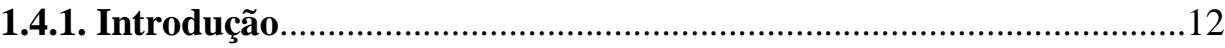

1.4.1.1. Significado Geológico de Inclusões Fluidas .............................12

1.4.1.2. Relações entre Inclusões Fluidas e Formação de Minério......12

1.4.2. Inclusões fluidas em depósitos de $\mathrm{Zn}$-Pb do tipo Mississippi Valley (MVT)

1.4.3. Inclusões fluidas em depósitos tipo Irish ……..................................17

1.4.4. Inclusões fluidas no Grupo Vazante: depósitos de Morro Agudo, Fagundes, Ambrósia e Vazante .................................................................17

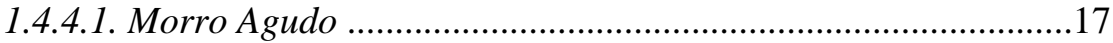

1.4.4.2. Fagundes …..........................................................................19

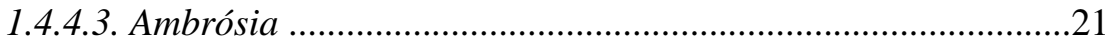

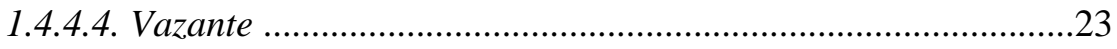

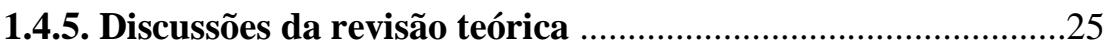

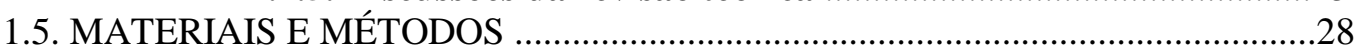

Capítulo 2

(ARTIGO A SER PUBLICADO): "Evolution of the Hydrothermal Systems in the Southern Extension of the Vazante Group: Implications to Silicate Zinc Mineralization"

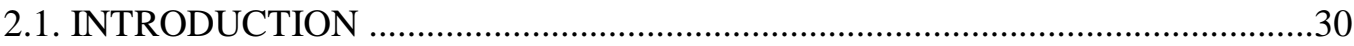

2.2. THE VAZANTE GROUP: GEOLOGICAL SETTING, STRATIGRAPHY AND

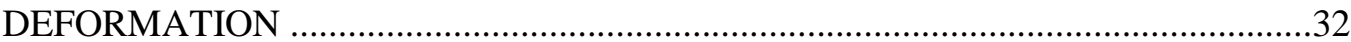

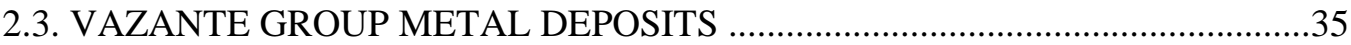

2.3 . PREVIOUS FLUID INCLUSION SUDIES IN THE VAZANTE GROUP............38

2.4. PREVIOUS CARBON AND OXYGEN ISOTOPIC STUDIES IN THE

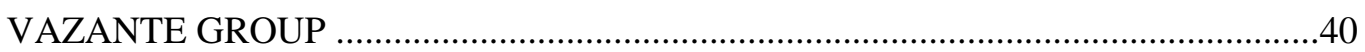

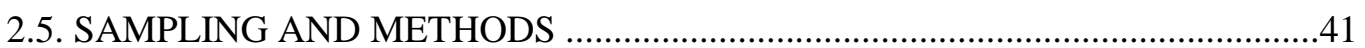

2.6. THE HYDROTHERMAL ALTERATIONS IN THE SERRA DO POÇO VERDE FORMATION IN BARREN AND MINERALIZED ZONES ...................................42

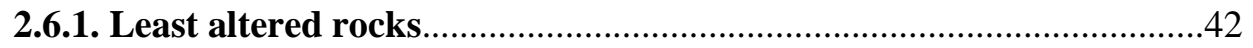

2.6.2. Diagenetic and hydrothermal alterations ...........................................4 44

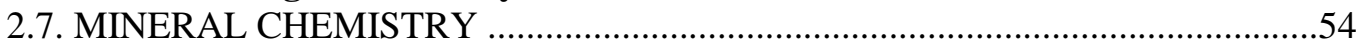

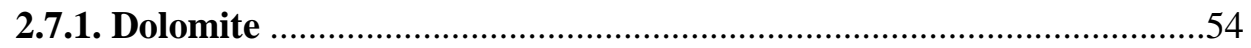

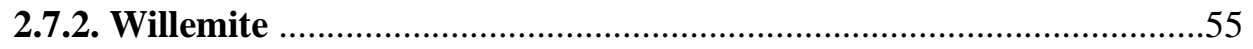

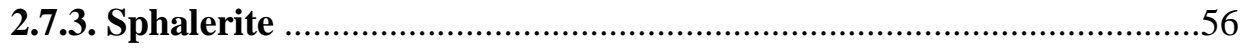

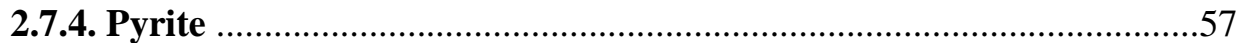

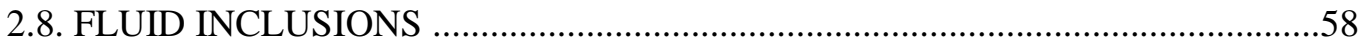

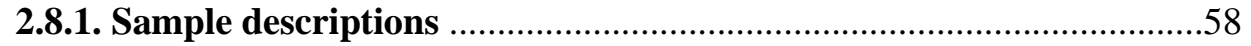

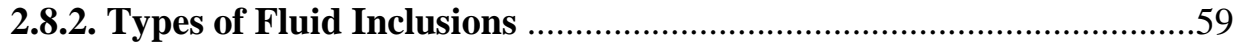

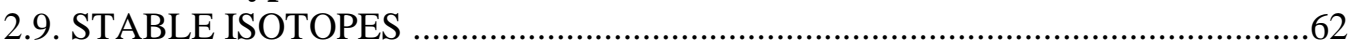




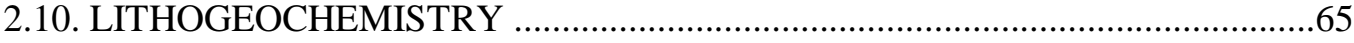

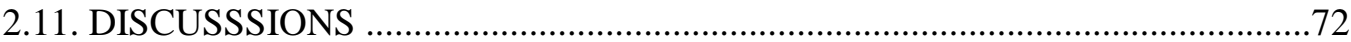

2.11.1. Evolution of the hydrothermal systems in the Southern Extension of

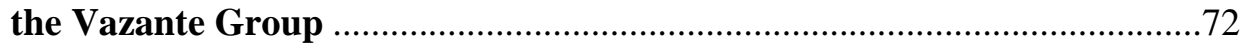

2.11.2. Comparison with other Vazante District Zn deposits .......................75

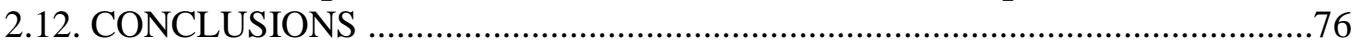

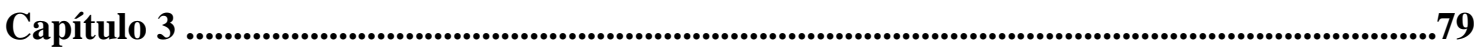

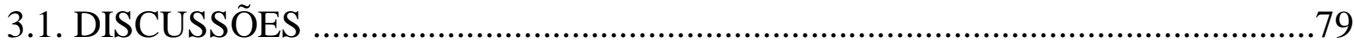

3.1.1. Evolução do sistema hidrotermal na Extensão Sul do Grupo Vazante

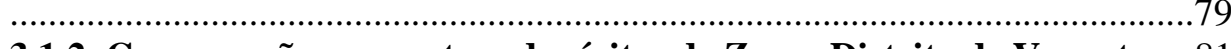

3.1.2. Comparação com outros depósitos de Zn no Distrito de Vazante ....8

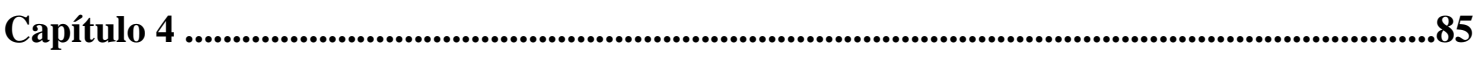

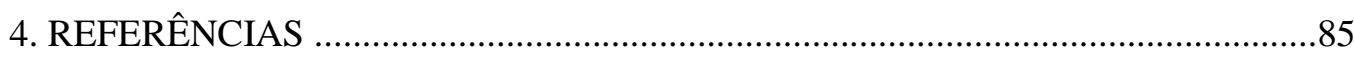

Apêndices......................................................................................................................................................93 


\section{LISTA DE FIGURAS}

Figura 1.1. A Província Tocantins e os elementos tectônicos do Brasil Central...............................................................

Figura 1.2. Unidades tectônicas da Faixa de Dobramentos Brasília...............................................................................6

Figura 1.3. A: Seção geológica esquemática da Faixa Brasília........................................................................................7

Figura 1.4. Mapa geológico do Grupo Vazante e localização dos depósitos de Vazante e Fagundes...............................10

Figura 1.5. Coluna litoestratigráfica esquemática (fora de escala) do Grupo Vazante...................................................11

Figura 1.6. Seção geológica esquemática W-E da região de Vazante............................................................................11

Figura 1.7. Fotomicrografia que exibe galena $(\mathrm{Gn})$ precipitada em zonas de crescimento em quartzo (Qz)...............13

Figura 1.8. Fotomicrografia de uma grande inclusão multifásica que contem alguns minerais filhos............................13

Figura 1.9. Histogramas de temperatura de homogeneização $(A)$ e salinidade $(B)$ de fluidos pré-mineralização para vários depósitos MVT ...................................................................................................................................15

Figura 1.10. Histogramas de temperatura de homogeneização (A) e salinidade (B) de fluidos do estágio mineralizante para vários depósitos MVT ...........................................................................................................16

Figura 1.11. Histogramas de temperatura de homogeneização (A) e salinidade (B) de fluidos pós-mineralização para

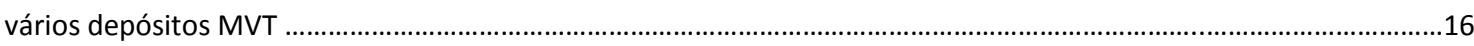

Figura 1.12. Localização dos sítios de amostragem ao longo de seção leste-oeste de Morro Agudo ..........................18

Figura 1.13. A: Histograma das temperaturas de fusão de gelo para inclusões fluidas primárias e pseudo-secundárias em esfaleritas do depósito de Morro Agudo

Figura 1.14. A: Histograma das temperaturas de homogeneização total ( $T_{h}$ ) para inclusões fluidas primárias e pseudo-secundárias em esfaleritas do depósito de Morro Agudo ..............................................................................19 Figura 1.15. Diagrama bivariante mostrando as relações entre as temperaturas de eutético ( $\left.T_{e}\right)$ e de fusão do gelo ( $\mathrm{Tfg}$ ) em diferentes gerações de inclusões fluidas do depósito de Fagundes ................................................................21

Figura 1.16. Diagrama mostrando a variação de $T_{h}$ e $T_{f g}$ em inclusões fluidas em diferentes fases do depósito de

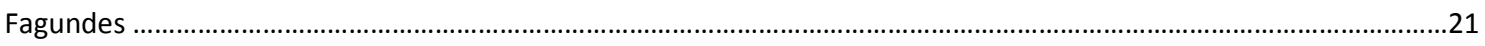

Figura 1.17. Diagrama que mostra a variação de $T_{h}$ e $T_{f g}$ em inclusões fluidas em diferentes fases do depósito de

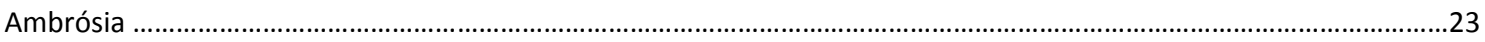

Figura 1.18. Variações das temperaturas de eutético $\left(T_{e}\right)$ e de fusão do gelo $\left(T_{f g}\right)$ em diferentes gerações de inclusões fluidas do Depósito de Vazante ...............................................................................................................24

Figura 1.19. Variações dos valores de The Tfg em inclusões fluidas em quartzo do depósito de Vazante....................25

Figura 1.20. Variação de Te e Tfg em inclusões fluidas dos depósitos de Vazante, Ambrósia e Fagundes ....................26

Figura 1.21. Variações dos valores de $T_{h}$ e $T_{f g}$ em inclusões fluidas dos depósitos de Vazante, Ambrósia e

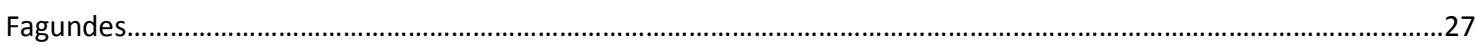

Figura 1.22. Comparação entre os dados de Th e Tfg em inclusões fluidas primárias dos depósitos de Fagundes,

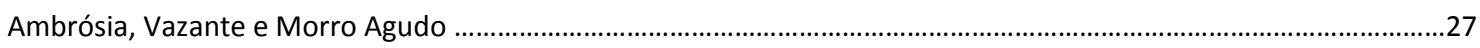

Fig. 2.1. Location map and geotectonic setting of the Vazante Group .....................................................................31

Fig. 2.2. Schematic diagram of the lithostratigraphic framework of the Vazante Group ............................................34

Fig. 2.3. Geological map of the Vazante Belt and the location the drill core studied ...................................................36

Fig. 2.4. Unaltered rocks from the drill core VZVG003 ..............................................................................................43

Fig. 2.5. Photomicrographs of the unaltered rocks from the studied drill core ...........................................................4

Fig. 2.6. Rock units and various styles of alteration documented along the drill core VZVG003 ..................................45

Fig. 2.7. Paragenetic sequence of the hydrothermal alterations identified ................................................................46

Fig. 2.8. Early Stage alteration in the Lower Pamplona Member ...............................................................................48

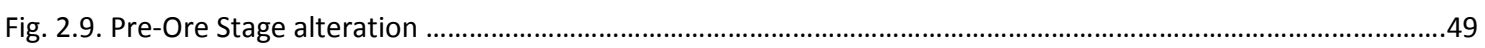

Fig. 2.10. Drill core samples showing the various events related to the ore stage alteration ......................................50

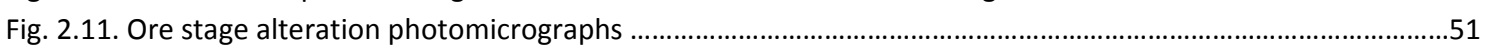

Fig. 2.12. Pyrite-bearing and galena-bearing alterations core samples ....................................................................52

Fig. 2.13. Pyrite-bearing and galena-beraing alterations photomicrographs ...........................................................53

Fig. 2.14. Late silicification photomicrographs and core sample ..............................................................................54

Fig. 2.15. Box and whisker plots showing the variation in composition of different generations of dolomite ...........55

Fig. 2.16. Box and whisker plots showing the variation in composition of different generations of willemite ...........56

Fig. 2.17. Box and whisker plots showing the variation of different generations of sphalerite ...................................57 
Fig. 2.18. $\mathrm{Cd}-\mathrm{Fe}-(\mathrm{Ag}+\mathrm{Ge}+\mathrm{Cu})$ atomic ratios for sphalerite from the sulfide alteration in the Upper Morro do Pinheiro and Lower Pamplona members compared to sphalerite from the Ambrósia, Fagundes and Vazante deposits

.. .57

Fig. 2.19. Box and whisker plots showing the variation of different generations of pyrite .........................................58

Fig. 2.20. Photomicrographs showing the different fluid inclusions types identified in the VZVG003 drill core .......59

Fig. 2.21. Salinity for the different fluid inclusions identified at the various alterations styles ..................................60

Fig. 2.22. Homogenization temperatures for the different fluid inclusions identified at the various alterations styles

. .61

Fig. 2.23. Binary diagrams of the oxygen and carbon isotope composition of (A) different occurrences of dolomite and the (B) calculated isotope compositions of the fluid in equilibrium with them .63

Fig. 2.24. Carbon and oxygen isotope values for the least altered dolostones and the different hydrothermal alteration styles throught the drill hole

Fig. 2.25. Box and whisker plots showing the variation in composition of the major elements for the various alteration styles

Fig. 2.26. Box and whisker plots showing the variation in composition of the elements from $\mathrm{Ba}-\mathrm{Ho}$ for the various alteration styles

Fig. 2.27. Box and whisker plots showing the variation in composition of the elements from La - Th for the various alteration styles

Fig. 2.28. Box and whisker plots showing the variation in composition of the elements from Mo - Se for the various alteration styles

Fig. 2.29. Box and whisker plots showing the variation in composition of the elements from Tm - Li for the various alteration styles

Fig. 2.30. Box and whisker plots showing the variation in composition of the elements $T e, T I, S$, and $C$ for the various alteration styles

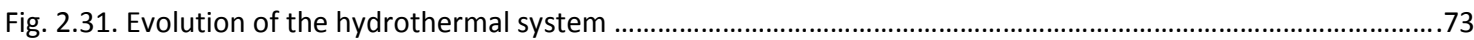

Fig. 2.32. Comparison of homogenization temperatures ( $\mathrm{Th}$ ) and salinities (wt. \% $\mathrm{NaCl}$ equiv.) of fluid inclusions from this study with other studies in different deposits 


\section{LISTA DE TABELAS}

Tabela 1.1. Fontes dos dados de inclusões fluidas de depósitos MVT típicos e atípicos ............................15

Tabela 1.2. Sumário dos dados microtermométricos do depósito de Fagundes...................................20

Tabela 1.3. Sumário dos dados microtermométricos do depósito de Ambrósia...................................20

Tabela 1.4. Sumário dos dados microtermométricos do depósito de Vazante.........................................23

Tabela 1.5. Padrões e limites de detecção para os elementos analisados em microssonda electronica..................28

Table 2.1. Major characteristics of hypogene sulfide lead-zinc and silicate zinc deposits and occurrences in the

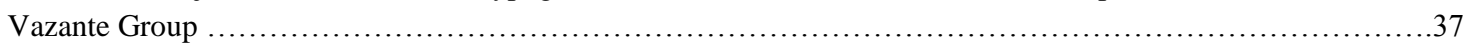

Table 2.2. Fluid inclusion characteristics from the zinc (and lead) deposits of the Vazante Group...................39

Table 2.3. Compilation of the stable isotope data from the zinc (and lead) deposits from the Vazante Group ........41

Table 2.4. Compositional range and average composition in (wt. \%) of different varieties of dolomite from the various alteration styles.

. .55

Table 2.5. Compositional range and average composition (in wt. \%) of different generations of willemite, sphalerite

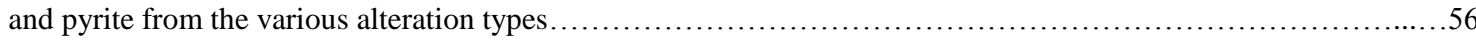
Table 2.6. Fluid inclusion data for the different fluid inclusion types identified in the various alteration styles in samples from the VZVG003 Drill Core. 


\section{Capítulo 1}

\subsection{INTRODUÇÃO}

Zinco é um metal de grande importância para indústria e saúde humana e é o quarto metal mais comum em uso, atrás somente do ferro, alumínio e cobre, com uma produção anual é de aproximadamente 12 milhões de toneladas (Tolcin, 2011). Este elemento é fortemente aplicado em galvanização, ligas metálicas e outras diversas aplicações industriais, como na indústria farmacêutica e química em geral. A principal fonte de zinco é na forma sulfetada (esfalerita - ZnS) e os mais importantes depósitos de zinco (e chumbo) estão hospedados em sequências sedimentares, como os depósitos dos tipos Mississippi Valley (MVT) e sedimentar exalativo (SEDEX). Juntos eles contêm em torno de $48 \%$ e $52 \%$ dos recursos globais de zinco e chumbo, respectivamente (Singer, 1995) e compõem o principal repositório destes metais na Terra, atualmente. Os depósitos do tipo Irish também são depósitos expressivos e exibem características que podem ser consideradas transicionais entre as outras duas classes descritas acima (Wilkinson, 2014).

Durante o século XIX e início do século XX, a principal fonte de zinco era proveniente de depósitos supergênicos não sulfetados, e smithsonita $\left(\mathrm{ZnCO}_{3}\right)$ era o principal mineral minério. Posteriormente, os depósitos sulfetados tornaram-se as principais fontes de zinco devido ao desenvolvimento de processos de flotação diferencial a das habilidades de fundição e refino de concentrados de esfalerita. Isso fez com que muitos depósitos com grande conteúdo de Zn não sulfetados, hospedados em óxidos, silicatos e carbonatos fossem ignorados (Hitzman et al., 2003). No entanto, os altos teores destes depósitos, o recente desenvolvimento metalúrgico de tratamento de minério não sulfetado e o menor impacto ambiental deste tratamento renovou o interesse comercial nestes depósitos de zinco, como uma potencial grande fonte deste metal no século XXI (Brugger et al., 2003; Hitzman et al., 2003; Large, 2001). Apesar do grande potencial dos depósitos de zinco não sulfetado, não existe uma classificação bem definida para

esse grupo de depósitos ${ }_{2}$ e pouco é conhecido a respeito dos processos relacionados à formação de depósitos hipogênicos, em particular.

O Grupo Vazante, inserido na Faixa de Dobramentos Brasília, hospeda os principais depósitos de zinco do Brasil (Morro Agudo, Ambrósia, Fagundes e Vazante) e é o distrito zincífero mais importante do Brasil (Dardenne, 2000). Este grupo hospeda as únicas minas ativas de zinco silicatado (Vazante) e zinco e chumbo sulfetado (Morro Agudo) do país, operadas pela Votorantim Metais S.A. (Oliveira, 2013). Ocorrências zincíferas são conhecidas em um raio de $15 \mathrm{~km}$ entorno da Mina de Vazante, considerada o maior depósito willemítico $\left(\mathrm{Zn}_{2} \mathrm{SiO}_{4}\right)$ do mundo, com recursos totais estimados de 40 a $60 \mathrm{Mt}$ a $20 \%$ de $\mathrm{Zn}$ (Baia, 2013). Apesar do recente interesse commercial nos depósitos de zinco silicatado, esta depósito já vem sendo explorado desde a decáda de 1960. O depósito de Zn sulfetado de Morro Agudo apresenta reservas medidas de 9,7 Mt @ 6,5\% de Zn e 2,8\% de Pb (Viviani et al., 2001). Várias ocorrências de zinco silicatado e sulfetado são relatadas no Grupo Vazante, entretanto, pouco é conhecido a respeito de suas relações genéticas com os grandes depósitos.

Muitos estudos foram desenvolvidos focados principalmente nas características mineralógicas, geoquímicas e estruturais do minério e de alterações hidrotermais relacionadas (Amaral, 1968a; 1968b; Bettencourt et al., 2001; Bez, 1980; Carvalho et al., 1962; Dardenne, 1979; Dardenne \& Freitas-Silva, 1999; Dardenne \& Schobbenhaus, 2001; Dresch, 1987; 
Hitzman et al., 1995; Hitzman 1997; Misi et al., 1996; 2000; Monteiro et al., 1998a; Pinho, 1990, Pinho et al., 1989; Rigobello et al., 1988; Romagna \& Costa, 1988; Slezak, 2012; Slezak et al., 2013) e menos estudos foram realizados valendo-se de inclusões fluidas e de geoquímica de isótopos estáveis nas zonas (Baia, 2013; Cunha, 1999; Cunha et al., 2000; 2001; Misi et al., 1997; 1999; Monteiro, 1997; 2002; Monteiro et al., 1998b; 1999; 2003; 2006; 2007; Olivo et al., 2014). Muito pouco, entretanto, é conhecido a respeito da história de interação fluido-rocha antes e depois do evento principal de mineralização e de suas características em zonas estéreis no Grupo Vazante.

\subsection{OBJETIVOS}

A finalidade desta investigação é documentar os vários estilos de alteração que ocorreram durante a evolução do Grupo Vazante (i. e. da diagênese à tardi-orogênese) no Alvo Varginha, nas rochas carbonáticas estéreis e mineralizadas dos membros Pamplona Inferior e Morro do Pinheiro Superior, da Formação Serra do Poço Verde. Esta unidade é a principal hospedeira dos depósitos de zinco, dessa maneira, esse estudo permitirá a comparação entre assinaturas de zonas mineralizadas e estéreis, trazendo novas perspectivas para o entendimento dos processos relacionados à formação de depósitos de zinco silicatado.

\subsection{CONTEXTO GEOLÓGICO REGIONAL}

\subsubsection{Província Tocantins}

A Província Tocantins, segundo Almeida et al., (1981), representa um orógeno de grandes dimensões que se desenvolveu durante o Neoproterozóico, em função da convergência e colisão de três importantes blocos. Estes blocos são o cráton do Amazonas a noroeste, o do São Francisco-Congo a leste e o suposto cráton do Paranapanema (Brito Neves et al., 1999), sotoposto aos sedimentos fanerozóicos da bacia do Paraná, a sudoeste (Figura 1.1). Este orógeno é formado pelas faixas de dobramentos Paraguai-Araguaia, que bordejam o Cráton do Amazonas, com vergência para o setor sudeste deste cráton, e Brasília, que bordeja o Cráton do São-Francisco, com vergência para o setor oeste deste cráton.

A configuração atual da província é estabelecida por núcleos cratônicos estabilizados durante o Trasamazônico (ca. 2,0 Ga), bordejados por cinturões de cavalgamentos e dobramentos desenvolvidos durante o Ciclo Brasiliano há 900 - 650 Ma (Almeida \& Hasui, 1984; Brito Neves et al., 1990; Schobbenhaus et al., 1981), sendo eles as faixas de dobramento Brasília, Rio Preto, Riacho do Pontal, Sergipana, Araguaia, Alto Rio Grande e Ribeira (Fuck et al., 1993). 


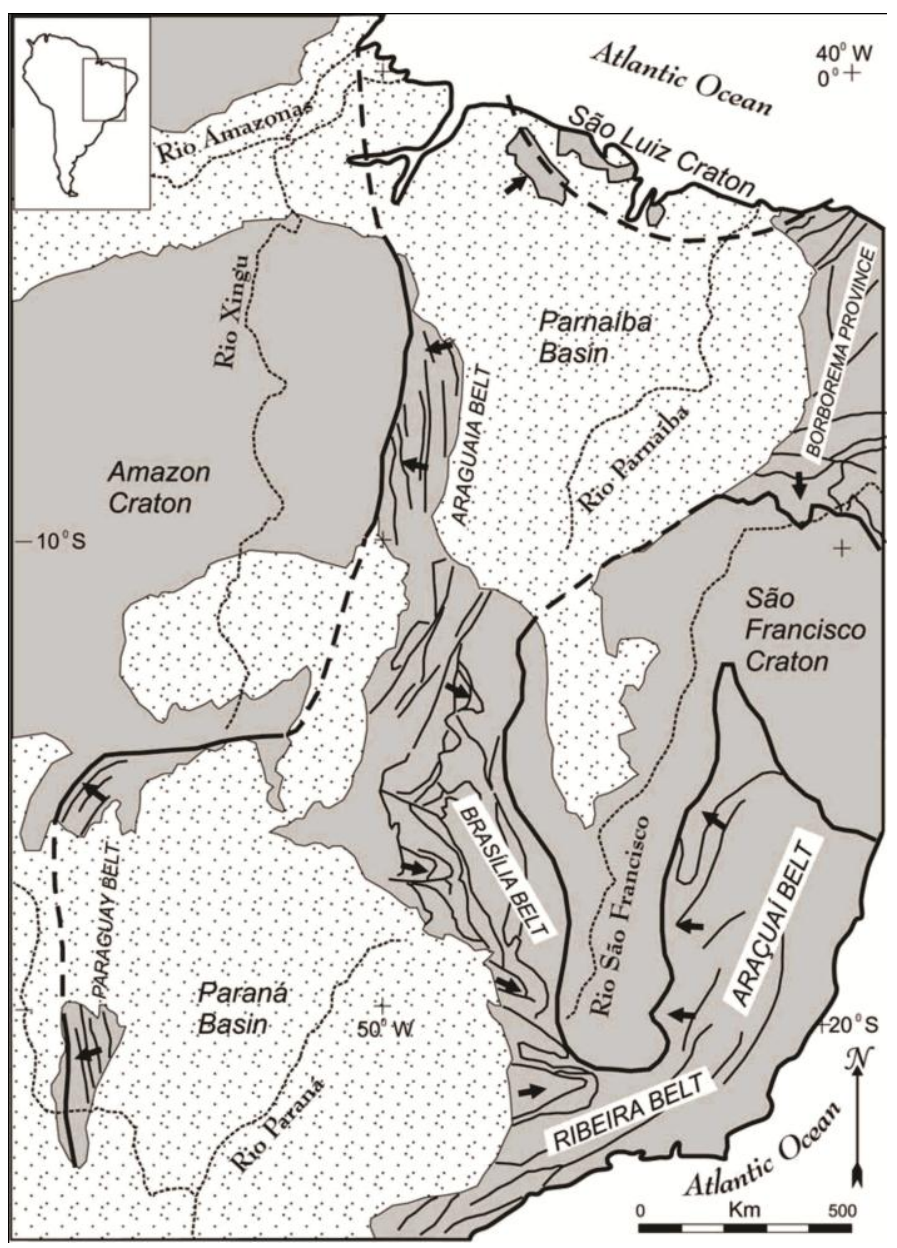

Figura 1.1. A Província Tocantins e os elementos tectônicos do Brasil Central, esboçado a partir de Delgado \& Pedreira (1995) por Valeriano et al., (2008). Linhas densas definem os limites dos blocos cratônicos. Setas indicam as principais direções de transporte tectônico ao longo dos cinturões orogênicos neoproterozóicos. Legenda: embasamento cristalino pré-cambriano em cinza; não metamorfisado em pontilhado.

\subsubsection{Faixa de Dobramentos Brasília}

\subsubsection{Aspectos Gerais}

A Faixa de Dobramentos Brasília (FDB) está situada na porção leste da Província Tocantins, às margens oeste do Cráton do São Francisco (CSF), e se estende por mais de 1000 $\mathrm{km}$ em direção aproximadamente N-S, desde o sul de Minas Gerais até o norte de Tocantins (Dardenne, 2000; Fuck, 1994; Marini et al., 1981). A FDB compreende terrenos e lascas de empurrões que convergem a leste contra a porção oeste da plataforma São Francisco-Congo (Valeriano et al., 2008). Segundo Dardenne (2000), as principais unidades da FDB (Figura 1.2) são:

- (i) Espessas sequências sedimentares e metassedimentares compreendidas pelos grupos Serra da Mesa (Paleo/Mesoproterozóico), Paranoá e Canastra (Meso/Neoproterozóico), Araxá, Ibiá e Vazante (Meso/Neoproterozóico), Bambuí (Neoproterozóico);

- (ii) Intrusões ígneas e sequências vulcano-sedimentares de várias idades, representados pelos complexos máfico-ultramáficos de Niquelândia, Cana Brava e Barro Alto (Paleo/Mesoproterozóico), granitos anorogênicos da sub-província do Rio Paranã e Rio Tocantins (Paleo/Mesoproterozóico), sequências vulcano-sedimentares de Juscelândia, 
Palmeirópolis e Indianópolis (Mesoproterozóico) e granitos pré, sin e pós-orogênicos e magmatismo máfico e ultramáfico (Neoproterozóico).

- (iii) O Arco Magmático de Goiás (Neoproterozóico), formado pelas sequências vulcano-sedimentares de Mara Rosa e Chapada e pelas rochas tonalíticas/granodioríticas de origem juvenil que ocorrem sobre vastas áreas da porção oeste da FDB.

Além dessas unidades descritas acima, a FDB envolve uma unidade mais antiga, conhecida como Maciço de Goiás, o qual inclui os terrenos granito-greenstone de Goiás Velho, Crixás, Guarinos e Pilar de Goiás; o embasamento granito-gnáissico paleoproterozóico de Cavalcante; as sequências vulcano-sedimentares paleoproterozóicas de Santa Terezinha, AlmasDianópolis e São Domingos; e a sequência metassedimentar Ticunzal.

Esse cinturão exibe um formato curvo com dois ramos de diferentes orientações: Faixa Brasília Norte, com trend de direção SW/NE, e a Faixa Brasília Sul, com trend de direção NW/SE. Essa concavidade pronunciada para leste é resultante da acomodação dos terrenos acrescidos em torno da protuberante margem oeste do CSF, o qual atuou com um anteparo de endentação. Dessa maneira, grandes porções do setor oeste do paleocontinente São Francisco estão atualmente cobertas por zonas de cavalgamento e nappes associadas à deformação de pele fina (thin-skinned), especialmente ao longo Faixa Brasília Sul (Valeriano et al., 2008). O Grupo Vazante, em conjunto com os grupos Ibiá e Canastra formam um complexo sistema de nappes que foram empurradas por cima dos sedimentos do Grupo Bambuí a leste durante a Orogênese Brasiliana (Dardenne, 2000).

\subsubsection{Configuração Tectônica da FDB}

Segundo Dardenne (2000), em geral, as principais unidades sedimentares e metassedimentares da FDB mostram deformação tectônica que é progressivamente mais intensa em direção oeste, acompanhada pelo aumento do grau metamórfico. Ocorre uma transição de sedimentos não metamorfisados na área cratônica a rochas em fácies anfibolito e até mesmo granulito na porção oeste da FDB. Essa evolução da deformação associada ao metamorfismo reflete a clara vergência da FDB em relação ao CSF. Essa zonação tectônica, inicialmente proposta por Costa \& Angeiras (1971), foi descrita por Dardenne (1978) e reformulada por Fuck et al., (1994), após a definição e individualização das Zona Interna, a oeste, Zona Externa, na região central, e Zona Cratônica (Zona Foreland), a leste.

Dardenne (2000) aponta a importância da mega-inflexão ou sintaxe dos Pirineus, destacada desde os estudos de Marini et al., (1981, 1984a, b). Ela é um lineamento WNW-ESE situada na mesma latitude do Distrito Federal que permite a subdivisão da FDB em segmentos Norte e Sul, que mostram singular evolução geotectônica, embora apresentem características muito distintas (Araújo Filho, 1999; Fonseca, 1996; Fonseca et al., 1995; Strieder, 1993).

No segmento norte da FDB, a maior parte das unidades sedimentares não foram metamorfisadas ou somente sofreram metamorfismo fácies xisto verde baixo. As suas relações estratigráficas permanecem bem preservadas, o que permite uma litoestratigrafia detalhada e uma reconstrução da paleogeografia e dos sistemas deposicionais. Essa característica é devido ao posicionamento em um nível crustal mais elevado do embasamento granito-gnáissico, que atuou como um bloco rígido a frente do trend compressivo do Ciclo Brasiliano (Dardenne, 2000). Na área situada a norte de Brasília, ocorrem as sequências sedimentares dos grupos Paranoá e Araí. Neste local, os efeitos da compressão são expressos pelo desenvolvimento de grandes falhas transcorrentes dextrais e empurrões que promoveram transporte do embasamento, mas somente afetaram localmente as coberturas sedimentares (Figura 1.3-A). As sequências metassedimentares que exibem grau metamórfico maior que fácies anfibolito 
ocorrem em áreas a oeste da megassutura. Essas rochas incluem os complexos máfico/ultramáficos de Niquelândia - Barro Alto - Cana Brava, metamorfisados em fácies granulito. Geralmente a vergência da deformação observada no segmento norte da FDB indica um trend compressivo principal com direção NW-SE (Araújo Filho, 1999; Fonseca \& Dardenne, 1995; Fonseca et al., 1995). De acordo com Araújo Filho (1999), o segmento norte da FDB foi empurrado sobre o segmento sul ao fim do Ciclo Brasiliano (Roscoe \& Araújo Filho, 1994).

Segundo Dardenne (2000), o segmento sul da FDB mostra distintas feições tectônicas, quando comparado ao segmento norte. A deformação e metamorfismo associado foram muito intensos, os quais obliteraram as relações estratigráficas entre as várias unidades (Figura 3-B). Os grupos Araxá, Canastra, Ibiá e Vazante foram envolvidos em um complexo sistema imbricado de nappes e empurrões que indicam transporte tectônico de grande magnitude, da ordem de dezenas a centenas de quilómetros. Os contatos entre as várias assembleias envolvidas correspondem a zonas de cisalhamento de baixo ângulo e frequentemente exibem a característica forma arqueada de dobras em bainha que desenvolveram rampas de cisalhamento lateral (Araújo Filho, 1999; Schimidt \& Fleischer, 1978; Seer, 1999; Seer et al., 1998; Simões, 1991; Simões, 1995; Simões \& Navarro, 1996; Simões \& Valeriano, 1990; Strieder, 1993, 1994; Teixeira \& Danni, 1978; Valeriano, 1992). Quanto mais próximo do CSF, a deformação e metamorfismo associado diminuem progressivamente, o que dá justificativa para a divisão da FDB em zonas Interna, Externa e Cratônica (Dardenne, 2000), as quais os contatos são marcados por grandes falhas regionais orientadas N-S (Dardenne, 1978). No geral, a vergência da deformação inicial observada no segmento sul da FDB indica um trend compressivo principal orientado de SW para NE. Isso é associado ao transporte predominante para SE, marcado por zonas regionais de cisalhamento transcorrente sinistral que mostram a mesma orientação (Araújo Filho, 1999; Seer, 1999; Valeriano et al., 1997). 


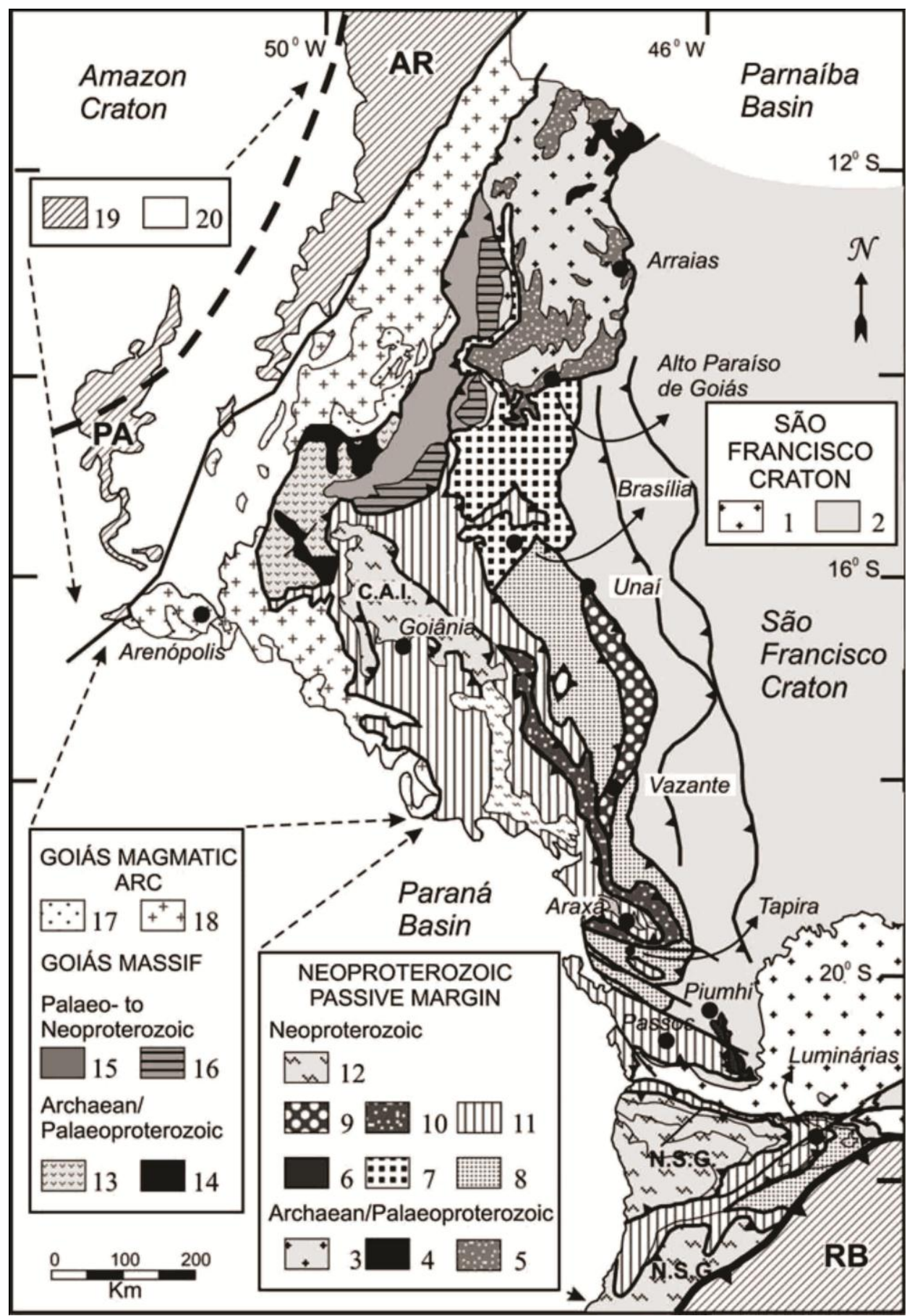

Figura 1.2. Unidades tectônicas da Faixa de Dobramentos Brasília (Dardenne, 2000, modificado por Valeriano et al., 2008). Zona Cratônica (Zona de Foreland): 1, terreno granito-greenstone e gnaisse-migmatítico arqueano/paleoproterozóico; 2, coberturas metassedimentares autóctones/parautóctones (grupos S. João del Rei, Carandaí, Andrelândia e Bambuí). Faixa Brasília (3 - 18): Zona Externa: 3, associação de granito-greenstones e gnaisse-migmatitos arqueano/paleoproterozóico; 4, cinturões greenstone arqueano/paleoproterozóico; 5, sucessões de rifte paleo- a mesoproterozóicas (Grupo Araí); 6, sistema de empurrões Ilicínea - Piumhi. Sucessões de margem passiva neoproterozóicas; 7, Grupo Paranoá; 8, unidades de quartzitos e filitos (grupos Canastra e Andrelândia) e ocasionais afloramentos de embasamento; 9, Grupo Vazante; 10, Grupo Ibiá; Zona Interna: 11, grupos Araxá e Andrelândia e rochas máficas toleíticas associadas, complexos de melanges ofiolíticas, pequenos afloramentos de embasamento e leucogranitos sin-colisionais; 12, nappes granulíticas (C.A.I., Complexo Anápolis-Itauçu; N.S.G., nappe Socorro-Guaxupé; Maciço de Goiás: 13, complexos granito-gnaisse-migmatíticos; 14, cinturões greenstone arqueano/paeloproterozóicos; 15, sucessões volcano-sedimentares mesoproterozóicas (Juscelândia, Palmeirópolis, Serra da Mesa); 16, complexos básico-ultrabásicos acamadados neoproterozóicos; Arco Magmático de Goiás: 17, rochas supracrustais (metavulcânicas/sedimentares) neoproterozóicas; 18, ortognaisses e granitoides neoproterozóicos; 19, cinturões Paraguai (PA), Araguaia (AR) e Ribeira (RB); 20, coberturas fanerozoicas. 

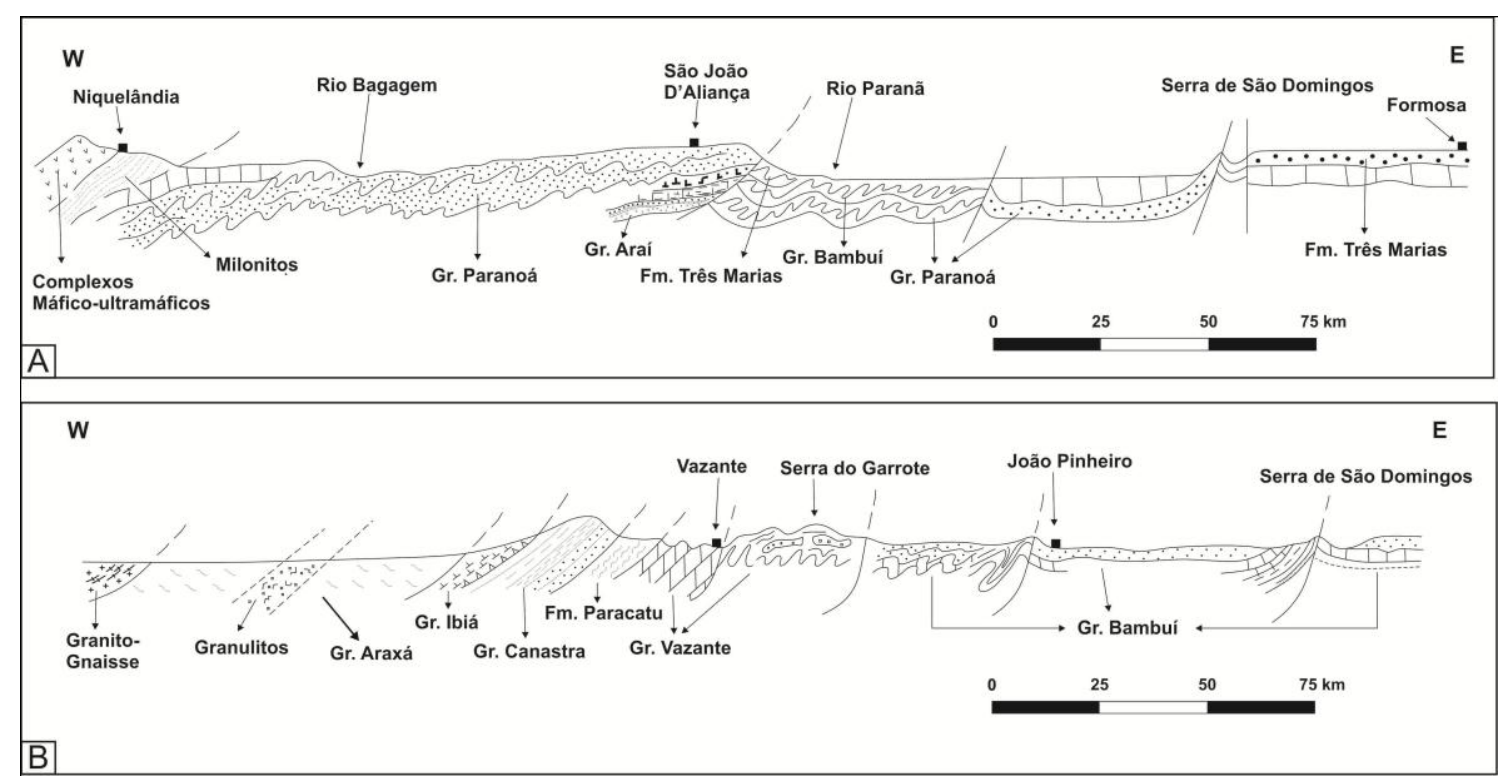

Figura 1.3. A: Seção geológica esquemática da Faixa Brasília entre Niquelândia e Formosa - Goiás, Brasil Central. B: Seção geológica esquemática que mostra as relações entre os grupos Araxá, Ibiá, Canastra, Vazante e Bambuí (Dardenne, 2000).

\subsubsection{Grupo Vazante}

O Grupo Vazante está localizado na parte noroeste do Estado de Minas Gerais, onde os metassedimentos desse grupo cobrem uma área de aproximadamente $250 \mathrm{~km}$ em direção geralmente N-S (Figura 1.4), desde Coromandel até Unaí, com largura média de $25 \mathrm{~km}$ a $30 \mathrm{~km}$ (Oliveira, 2013). A geometria da faixa tem forma de arco com concavidade voltada para W e pode ser dividida em dois setores com aspectos estratigráficos, estruturais e metalogenéticos distintos, separados pela flexão do Rio Escuro. Na porção norte ocorrem os depósitos de Zn e $\mathrm{Pb}$ sulfetados de Morro Agudo, Ambrósia e Fagundes e no setor sul encontra-se a mineralização de $\mathrm{Zn}$ representada predominantemente por minério willemítico (Oliveira, 2013).

Os metassedimentos do Grupo Vazante são compostos por uma espessa sequência marinha pelito-dolomítica que pode ser dividida em sete formações (Figura 1.5), da base para o topo: Formação Santo Antônio do Bonito, Formação Rocinha, Formação Lagamar, Formação Serra do Garrote, Formação Serra do Poço Verde, Formação Morro do Calcário e Formação Serra da Lapa, descritas a seguir (Dardenne, 2000; Dardenne, et al., 1998):

- Formação Santo Antônio do Bonito: também conhecida como Formação Retiro é considerada a formação basal, consiste de camadas métricas de quartzito branco, localmente conglomerático, intercalado com ardósia. Nos rios Santo Antônio do Bonito e Santo Inácio, essa formação é caracterizada pela presença de diamictitos com seixos de quartzito, calcário, dolomito, metassiltito e granitóides, em uma matriz pelítica que é localmente fosfática. Maiores concentrações de fosfato são encontradas nas fácies ardosiana e em camadas de fosfarenito, ricos em intraclastos e pellets (Fosfarenito 1). As camadas de diamictito representam fluxo de detritos depositados em águas relativamente profundas por correntes de gravidade (Dardenne et al., 1998; Souza, 1997);

- Formação Rocinha: na base essa formação consiste de sequências de ritmitos arenosos e pelíticos que gradam da Formação Santo Antônio do Bonito, ascendentemente. Na sua parte superior, ela compreende espessa sequência de camadas de ardósias e metassiltitos regularmente intercaladas com alterações de coloração amarela e vermelha. Essa sequência passa verticalmente de ardósia cinza escura com carbonato e pirita, com laminações 
fosfáticas finas gradualmente para fosfarenitos ricos em intraclastos e pellets (Fosfarenito 2), que formam o depósito de fosfato de Rocinha, o qual apresenta reservas da ordem de $400 \mathrm{Mt}$ a $12 \% \mathrm{P}_{2} \mathrm{O}_{5}$. Na porção superior da formação, sedimentos rítmicos (quartzito e siltito) hospedam o depósito de fosfato de Lagamar (Dardenne, 2000; Dardenne et al., 1997; Nogueira, 1993), compostos essencialmente por fosfarenitos (Fosforito 3). Reservas deste depósito foram estimadas em $5 \mathrm{Mt}$ a $25 \%$ de $\mathrm{P}_{2} \mathrm{O}_{5}$;

- Formação Lagamar: a unidade carbonato-psamo-pelítica de Lagamar (Campos Neto, 1984a, b; Dardenne, 1978a, b, 1979; Dardenne et al., 1976; Dardenne et al., 1997, 1998) é representada na porção basal pela alternância de camadas de conglomerado, quartzito, metassiltito e ardósia. As unidades de conglomerado exibem um arcabouço suportado por clastos de quartzito, metassiltito e calcário cinza escuro e são conhecidas como Membro Arrependido. Essas camadas psamíticas são recobertas por brechas dolomíticas intraformacionais que gradam para unidades de calcários cinza escuro, estratificados com intercalações de brecha lamelar seguidas de dolomitos estromatolíticos, conhecidos como Membro Sumidouro. As camadas estromatolíticas formam biohermas beges a rosadas, compostas por dolomito laminado (esteiras de cianobactérias), dolarenitos oncolíticos e doloruditos, e estromatólitos colunares com laminações convexas e cônicas do tipo Conophyton e Jacutophyton. Lateralmente e verticalmente essas biohermas interdigitam-se com camadas de metassiltitos carbonáticos e ardósia;

- Formação Serra do Garrote: essa formação consiste de uma espessa sequência de ardósia cinza escuro a cinza esverdeado, localmente rítmica, carbonosa e que contem pirita, com intercalações de quartzito (Campos Neto, 1984; Dardenne, 1978; Dardenne et al., 1997; 1998; Madalosso, 1980; Madalosso \& Valle, 1978);

- Formação Serra do Poço Verde: essa formação corresponde a uma sequência predominantemente dolomítica, primeiramente definida por Dardenne (1978a, b, 1979) e subsequentemente atribuída à Formação Vazante por Rigobello et al. (1988). Ela é dividida em quatro membros descritos sucessivamente da base para o topo:

- Membro Morro do Pinheiro Inferior: esse membro compreende dolomito laminado cinza claro a rosa com esteiras de cianobactérias, intercalados com níveis de dolarenito e brecha intraformacional associada com lentes de dolomito com estromatólitos colunares (espessura de aproximadamente $500 \mathrm{~m}$ );

-Membro Morro do Pinheiro Superior: esse membro consiste de dolomito cinza a cinza escuro, laminado, com esteiras de cianobactérias e frequentes feições de bird's eyes, intercalado com algumas camadas de dolarenito, brecha lamelar e folhelho carbonoso (espessura entre $300 \mathrm{~m}$ a $500 \mathrm{~m}$ );

- Membro Pamplona Inferior: constituído por camadas de siltitos cinza, verde e roxo intercalados com dolomito micrítico, rosa, laminado, com esteiras de cianobactérias e pequenas lentes de arenito fino a conglomerático (espessura entre $100 \mathrm{~m}$ a $200 \mathrm{~m}$ );

- Membro Pamplona Médio: esse membro consiste de dolomito cinza claro a rosa, com laminações de esteiras de cianobactérias, intercalado com camadas de dolarenito, brecha lamelar e dolomito com estromatólitos colunares, e com lentes de folhelho (espessura de aproximadamente $400 \mathrm{~m}$ );

- Formação Morro do Calcário: essa formação corresponde ao membro Pamplona Superior de Rigobello et al., (1988). É caracterizada pela presença de dolomito estromatolítico rosa, que forma biostromas e biohermas colunares com laminação convexa, associado a dolarenito oolítico e oncolítico e dolorudito (espessura de aproximadamente $200 \mathrm{~m}$ a 300 m). Na região de Morro Agudo, Paracatu e Unaí, a Formação Morro do Calcário excede $900 \mathrm{~m}$ de espessura, sendo composta essencialmente por dolorudito, que indica 
retrabalhamento de biohermas estromatolíticas parcialmente preservadas e associadas a fácies de dolarenitos oolíticos e oncolíticos. Essa espessura incomum sugere que, nesta região, as formações Morro do Calcário e Serra do Poço Verde representam uma única sequência dolomítica, depositada continuamente, que não permite a subdivisão definida para a região de Vazante;

- Formação Serra da Lapa: essa formação constitui a parte superior do Grupo Vazante, a qual foi descrita por Madalosso \& Valle (1978) e Madalosso (1980) na região de Paracatu, assim como os membros Serra do Velosinho e Serra da Lapa. Estes são representados por filitos carbonosos, metassiltitos com carbonato, lentes de dolomito e camadas de quartzito. As lentes dolomíticas exibem várias fácies como dolomito laminado com esteiras de cianobactérias, dolomito com estromatólitos colunares e dolomito com brechas intraformacionais, interdigitados com a sequência pelítica que cobre regionalmente as formações dominantemente dolomíticas do Morro do Pinheiro e Serra do Poço Verde.

A idade do Grupo Vazante ainda não é conhecida, embora informações obtidas a partir de estromatólitos colunares tipo Conophyton indicam um longo intervalo de idade entre 1,35 Ga e $950 \mathrm{Ma}$ (Cloud \& Dardenne, 1973; Dardenne et al., 1976; Moeri,, 1972), o que sugere uma correlação com o Grupo Paranoá (Dardenne, 2000).

Misi et al., (2014) propuseram que a parte superior da sequência sedimentar do Grupo Vazante é de idade mesoproterozóica e a parte basal é neoproterozóica, baseados em idades ReOs de folhelhos ricos em matéria orgânica (Azmy et al.,2008; Geboy et al., 2013) e em zircões detríticos dispersos ao longo da seção (Azmy et al., 2008; Rodrigues et al., 2012). É sugerido que a seção superior (da Formação Lagamar a Serra da Lapa) foi empurrada sobre a porção inferior (formações Santo Antônio do Bonito e Rocinha; Figura 1.6).

Segundo Oliveira (2013), a interpretação do contexto geotectônico do Grupo Vazante é controversa. Este é considerado como depósito de margem continental passiva (Almeida, 1993; Campos-Neto 1984a, b). Alkimin \& Martins-Neto (2012) consideram que o Grupo Vazante é uma cobertura cratônica de margem passiva neoproterozóica, correlata à sequência deposicional de $1^{\text {a }}$ ordem das bordas do Cráton do São Francisco, denominada Sequência Macaúbas (Oliveira, 2013). 


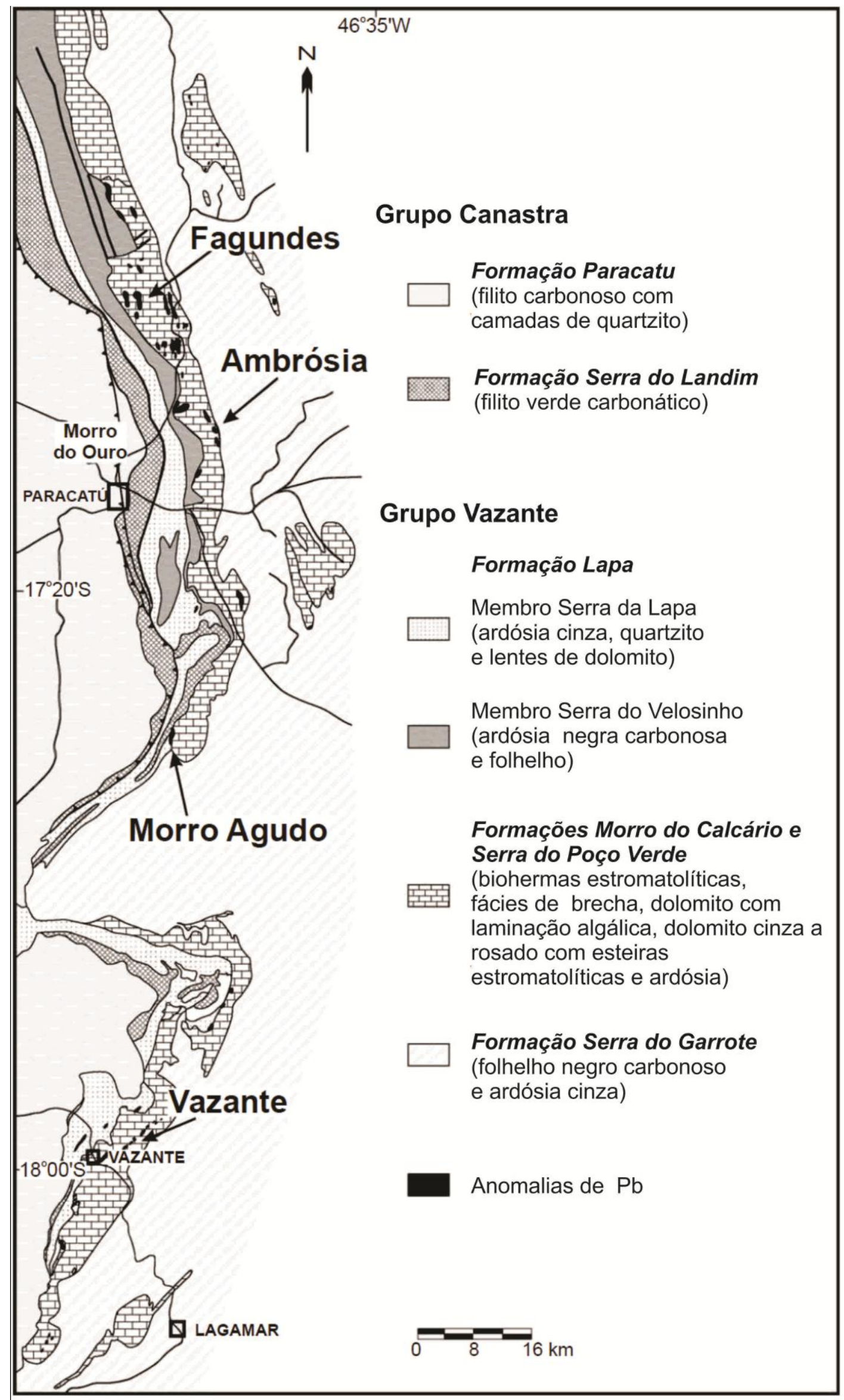

Figura 1.4. Mapa geológico do Grupo Vazante e localização dos depósitos de Vazante e Fagundes (Cia. Mineira de Metais, Votorantim Metais; Monteiro et al., 2006). 


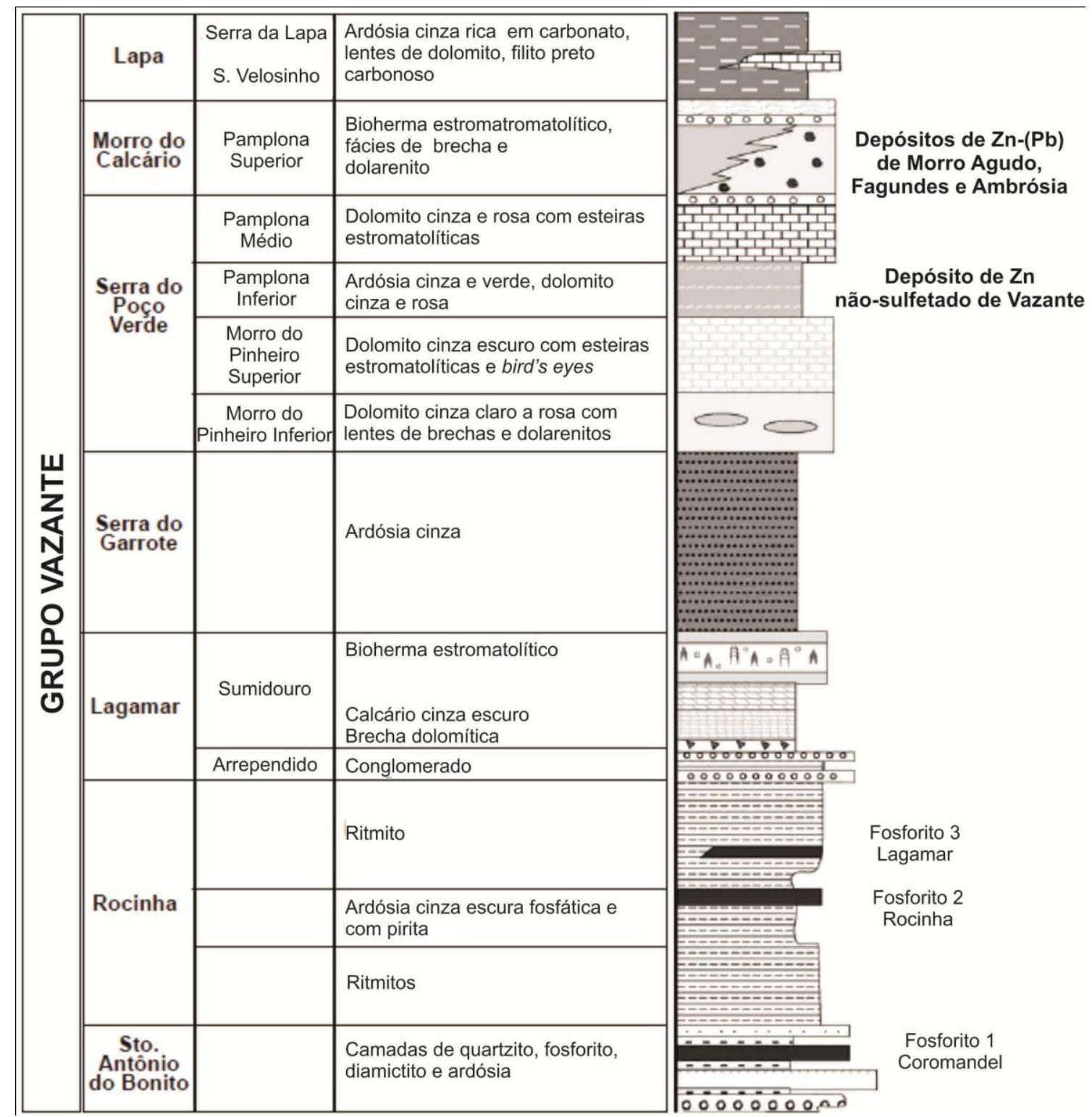

Figura 1.5. Coluna litoestratigráfica esquemática (fora de escala) do Grupo Vazante (Dardenne et al., 1998; Dardenne, 2000; Monteiro et al., 2006).

Vazante Gr. (1.3 to $1.1 \mathrm{Ga})$

Bambui Gr. (0.74 to $0.62 \mathrm{Ga})$

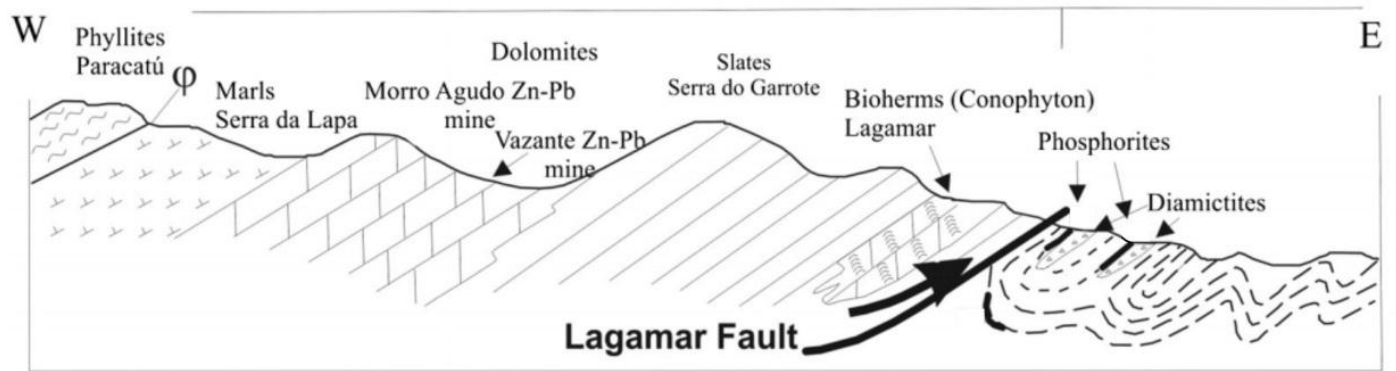

Figura 1.6. Seção geológica esquemática W-E da região de Vazante (extraída de Misi et al., 2014). 


\subsection{REVISAO DOS ESTUDOS DE INCLUSÕES FLUIDAS ASSOCIADAS A DEPÓSITOS DE METAIS BÁSICOS EM BACIAS SEDIMENTARES}

\subsubsection{Introdução}

Inclusões fluidas são cavidades desenvolvidas em imperfeições de cristais preenchidas por fluidos, por vezes sólidos, e estudá-las é a maneira mais direta de examinar fluidos que outrora estavam presentes em ambientes diagenéticos e hidrotermais. Inclusões fluidas podem ser compreendidas como cápsulas do tempo, armazenando informações sobre antigas temperaturas, pressões e composições de fluidos (Goldstein \& Reynolds, 1994).

Esta revisão reúne as características das inclusões fluidas estudadas em depósitos de metais básicos associados a bacias sedimentares, especialmente os de $\mathrm{Zn} \pm \mathrm{Pb}$ do tipo Mississippi Valley (MVT), típicos e atípicos, do tipo Irish e os depósitos de Morro Agudo, Fagundes, Ambrósia e Vazante.

\subsubsection{Significado Geológico de Inclusões Fluidas}

O fundamento básico no estudo de inclusões fluidas é que o fluido aprisionado deva ser homogêneo e represente o fluido associado à cristalização ou recristalização de um mineral. É importante, portanto, identificar evidências de processos como aprisionamento de fluidos heterogêneos, reequilíbrio com as superfícies da inclusão e necking down, além da diferenciação entre inclusões primárias, secundárias e pseudo-secundárias.

Inclusões secundárias, segundo Shepherd et al., (1985), geralmente são consideradas pouco importantes nos estudos, mas podem fornecer importantes informações a respeito de caminhos preferenciais de migração de fluidos assim como locais onde a atividade de fluidos foi mais pronunciada.

De acordo com Shepherd et al., (1985), inclusões fluidas tradicionalmente são usadas como geotermômetros, mas também são bons guias para avaliar as condições de temperatura e pressão, quando essa técnica é empregada em conjunto com outros geotermômetros e geobarômetros baseados em estabilidade mineral e equilíbrio líquido-sólido, incluindo fracionamento isotópico. Por essas razões o estudo de inclusões fluidas tem sido bastante utilizado na exploração mineral a partir da caracterização de assembleias minerais particulares associadas a minerais de minério e ganga, assim sendo possível prever determinadas condições de temperatura e pressão que favorecem a precipitação de corpos mineralizados.

\subsubsection{Relações entre Inclusões Fluidas e Formação de Minério}

Em um estudo de inclusões fluidas, é fundamental estabelecer a relação temporal entre as diferentes assembleias de inclusões e segundo Roedder \& Bodnar (1997), a maioria das inclusões na maioria das amostras pode ser presumida como secundária, ao menos que se prove o contrário. Muitas vezes em depósitos hidrotermais, onde é comum a superposição de várias fases de alteração, esse trabalho pode se mostrar inconclusivo.

A relação das inclusões estudadas com o processo de interesse é um dos critérios mais importantes neste tipo de estudo em depósitos minerais, ainda que receba atenção inadequada (Wilkinson, 2001). Muitas vezes é assumido que inclusões primárias ou pseudo-secundárias relacionadas a minerais de ganga espacialmente associados aos minerais de minério sejam representativas do fluido mineralizante. As evidências texturais de coprecipitação de fases minerais, no entanto, são frequentemente documentadas de maneira insatisfatória. Até mesmo 
relações de bordas de grãos em aparente equilíbrio e intercrescimento mineral não são provas de deposição simultânea (Wilkinson, 2001). Campbell \& Panter (1990) mostraram, usando microscopia de iluminação infravermelha, que inclusões em quartzo intercrescidos com cassiterita e wolframita apresentavam diferentes propriedades microtermométricas daquelas hospedadas nos minerais de minério.

Talvez as melhores evidências de relação sin-genética entre minerais de minério e de ganga sejam micro-inclusões de minerais de minério em minerais de ganga (Figura 1.7) e inclusões de minerais de minério em inclusões fluidas em minerais de ganga (Figura 1.8). Quando essas relações não são observadas, deve-se manter em mente que há uma incerteza em relação ao equilíbrio entre as fases minerais.

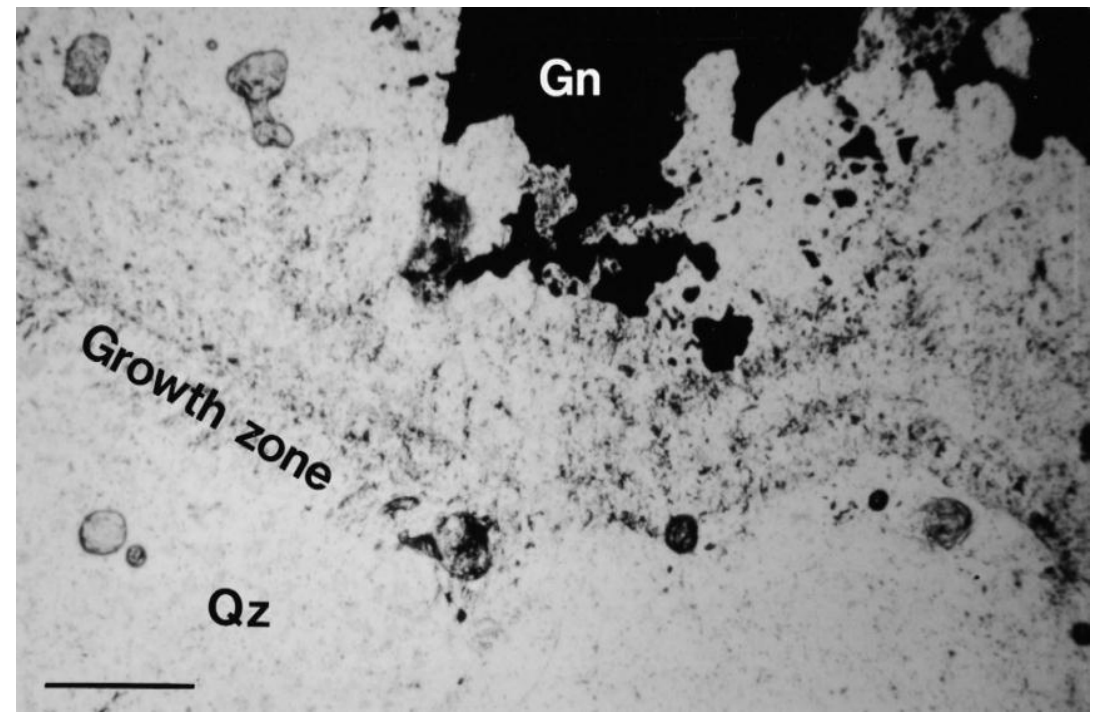

Figura 1.7. Fotomicrografia que exibe galena $(\mathrm{Gn})$ precipitada em zonas de crescimento em quartzo (Qz), definida por bandas de inclusões fluidas primárias. Veio mineralizado em $\mathrm{Pb}-\mathrm{Zn}$ de Crosscourse, Porthleven, sudoeste da Inglaterra. Luz plano-polarizada, barra de escala $2 \mathrm{~mm}$ (Wilkinson, 2001).

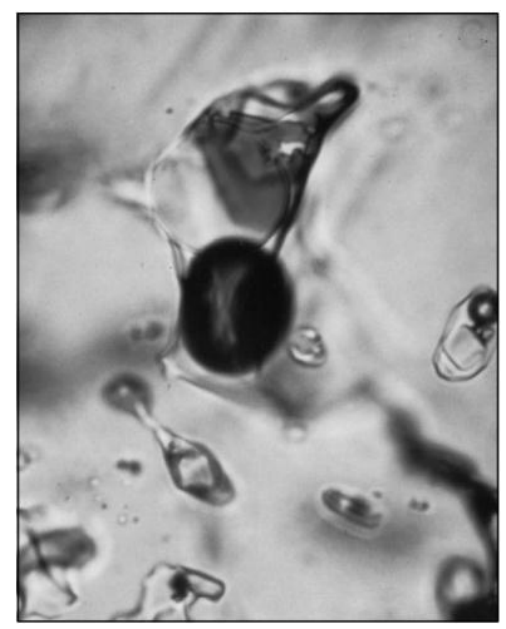

Figura 1.8. Fotomicrografia de uma grande inclusão multifásica que contem alguns minerais filhos, incluindo uma hematita vermelha, hexagonal. Plúton Capitan mineralizado em Th-U-ETR, Novo México. Luz plano-polarizada, espessura da imagem, $250 \mu \mathrm{m}$. Foto: cortesia de S. Mulshaw (Wilkinson, 2001). 


\subsubsection{Inclusões fluidas em depósitos de Zn-Pb do tipo Mississippi Valley (MVT)}

O estudo de inclusões fluidas em depósitos do tipo MVT foi realizado em uma série de minerais, principalmente calcita, dolomita, quartzo, barita, fluorita e esfalerita. De acordo com Wilkinson (2001), são inclusões relativamente simples, geralmente compreendendo inclusões aquosas bifásicas (líquido + vapor) com pequeno volume de vapor $(<10 \%$ do volume da inclusão), algumas vezes acompanhadas de inclusões aquosas monofásicas ou de hidrocarbonetos.

A primeira fusão é clara e irrompe em torno de $-55^{\circ} \mathrm{C}$ a $-50^{\circ} \mathrm{C}$, que corresponde a fusão eutética estável do sistema $\mathrm{NaCl}-\mathrm{CaCl}_{2}-\mathrm{H}_{2} \mathrm{O}$ (Wilkinson, 2001), e medidas das temperaturas de última fusão, sejam de hidrato ou gelo, permitem estimar a razão $\mathrm{NaCl} / \mathrm{CaCl}_{2}$ (e.g. Shepherd et al., 1985).

A raridade de cristais de $\mathrm{NaCl}$ indica que quantidades apreciáveis de outros cátions (além do $\mathrm{Na}^{+}$) devem estar presentes no fluido (Monteiro, 2002). A abundância relativa de solutos é estimada, na maioria dos depósitos, como $\mathrm{Cl}>\mathrm{Na}>\mathrm{Ca}>>\mathrm{K}>\mathrm{Mg}>\mathrm{B}$ (Roedder, 1984).

De acordo com Wilkinson (2001), inclusões fluidas ajudaram a definir parâmetros importantes a respeito da gênese de depósitos MVT, como alta densidade dos fluidos (>1000 kg $\mathrm{m}^{-3}$ ) que excede a densidade de muitos fluidos crustais e gera importantes implicações para questões de mecanismos de fluxo. Foram definidas pressões de fluido que geralmente são consideradas maiores que as pressões de vapor, o que não permite efervescência do fluido (boiling), embora exceções tenham sido relatadas (e.g. Jones \& Kesler, 1992). Segundo Roedder (1984), gases podem ter se mantido solúveis em pressões elevadas, como aproximadamente 20 bars.

De acordo com Monteiro (2002), matéria orgânica é frequentemente observada e sua presença tem um papel importante, devido a sua capacidade redutora, que promove a redução de sulfatos em sulfetos. Roedder (1984) ressalta que agentes complexantes de metais pesados usualmente estão presentes na matéria orgânica.

Basuki \& Spooner (2002) compilaram vários dados de temperatura de homogeneização $\left(\mathrm{T}_{\mathrm{h}}\right)$ e salinidade, e desenvolveram histogramas de frequência relativa com intervalos de temperatura e salinidade delimitados em classes de intervalos de $10^{\circ} \mathrm{C}$ e $2 \%$ em peso de $\mathrm{CaCl}_{2}$ equivalente para vários depósitos MVT típicos e atípicos. Os critérios adotados pelos autores para depósitos MVT típicos são calcários dolomíticos ou calcíticos, com deposição metálica epigenética e sem relação com atividade ígnea. Os fluidos associados ao estágio de precipitação de minério exibem temperaturas de homogeneização $\left(\mathrm{T}_{\mathrm{h}}\right)$ com valores que flutuam entre $\sim 75^{\circ}$ $\mathrm{C}$ a $\sim 150^{\circ} \mathrm{C}$ e com salinidades médias em torno de $\sim 15 \%$ a $\sim 30 \%$ em peso de $\mathrm{CaCl}_{2}$ equivalente. Esfalerita e/ou galena são as principais fases minerais de minério, com fluorita e minerais de sulfato presente somente em menores quantidades. As fontes dos dados utilizados por Basuki \& Spooner (2002) estão dispostos na Tabela 1.1. Algumas fontes fornecem dados de salinidade para os sistemas $\mathrm{NaCl}-\mathrm{H}_{2} \mathrm{O}$ e NaCl-CaCl $2-\mathrm{H}_{2} \mathrm{O}$ e esses dados de salinidade foram convertidos para o sistema $\mathrm{CaCl}_{2}-\mathrm{H}_{2} \mathrm{O}$. Para mais detalhes vide Basuki \& Spooner (2002). 
Tabela 1.1. Fontes dos dados de depósitos MVT típicos e atípicos utilizados no estudo de Basuki \& Spooner (2002).

\begin{tabular}{|c|c|c|c|c|c|}
\hline No. & Location & Type of Data & $\begin{array}{l}\mathrm{Zn}+\mathrm{Pb} \\
\text { (tons) }\end{array}$ & $\% \mathrm{Zn}+\mathrm{Pb}$ & Source \\
\hline \multicolumn{6}{|c|}{ Typical MVT } \\
\hline 1 & Elmwood-Gordonsville, Central Tennessee, U.S.A. & Linked Th and salinity & 2500000 & 3.6 & $\begin{array}{l}\text { Misra and Gratz (1988), Misra and } \\
\text { Lu (1992), Misra et al. (1996) }\end{array}$ \\
\hline 2 & Mascot-Jefferson City, East Tennessee, U.S.A. & Linked Th and salinity & 2500000 & $2-4$ & $\begin{array}{l}\text { Long et al. (1998), Misra and Ful- } \\
\text { weiler (1995), Taylor et al. (1983) }\end{array}$ \\
\hline 3 & Viburnum Trend, Southeast Missouri, U.S.A. & Linked Th and salinity & 12012000 & 6.6 & $\begin{array}{l}\text { Hagni (1983, 1995), Roedder } \\
\text { (1977), Shelton et al. (1992) }\end{array}$ \\
\hline 4 & Pine Point, Northwest Territories, Canada & Unlinked Th and salinity & 1400000 & 9.4 & Roedder (1968), Sangster (1996) \\
\hline 5 & Jubilee, Nova Scotia, Canada & Linked $\mathrm{Th}$ and salinity & 65340 & 6.6 & Chi et al. (1995) \\
\hline 6 & Gays River, Nova Scotia, Canada & Linked $\mathrm{Th}$ and salinity & 357600 & 14.9 & $\begin{array}{l}\text { Chi and Savard (1995), Sangster } \\
(1996)\end{array}$ \\
\hline 7 & Nanisivik, Nunavut, Canada & Linked $\mathrm{Th}$ and $\mathrm{Tm}$ & 965250 & 11 & $\begin{array}{l}\text { McNaughton (1983), Sutherland } \\
\text { and Dumka (1995) }\end{array}$ \\
\hline 8 & Polaris, Nunavut, Canada & Linked Th and salinity & 4356000 & 18 & $\begin{array}{l}\text { Randell (1994), Sangster (1996), } \\
\text { Savard et al. (2000) }\end{array}$ \\
\hline 9 & Coxco, McArthur River District, Australia & Unlinked $\mathrm{Th}$ and salinity & 60000 & 3 & Walker et al. (1983) \\
\hline 10 & Tri-State, U.S.A. & Linked $\mathrm{Th}$ and $\mathrm{Tm}$ & 27000000 & 3 & $\begin{array}{l}\text { Wei (1975), Ragan (1996), Sang- } \\
\text { ster (1996) }\end{array}$ \\
\hline 11 & Eastern Belgium & Linked $\mathrm{Th}$ and salinity & & & Muchez et al. (1994) \\
\hline 12 & Bongara, Northern Peru & Linked $\mathrm{Th}$ and salinity & & 15.8 & $\begin{array}{l}\text { Chi and Savard (1998a, 1998b; } \\
\text { unpublished data), www.solitari- } \\
\text { oresources.com }\end{array}$ \\
\hline 13 & Silesian District, Poland & Ranges of $\mathrm{Th}$ and salinity & & & Leach et al. (1996) \\
\hline 14 & San Vicente, Central Peru & Ranges of $\mathrm{Th}$ and salinity & & & $\begin{array}{l}\text { Fontboté and Gorzawski (1990), } \\
\text { Moritz et al. (1996) }\end{array}$ \\
\hline 15 & Upper Mississippi Valley, Wisconsin, U.S.A. & Unlinked $\mathrm{Th}$ and $\mathrm{Tm}$ & 2300000 & 3.5 & $\begin{array}{l}\text { Long et al. (1998), McLimans } \\
\text { (1977) }\end{array}$ \\
\hline 16 & Monte Cristo mine, North Arkansas, U.S.A. & Linked $\mathrm{Th}$ and salinity & 27971 & & Long et al. (1986) \\
\hline 17 & Metaline District, Washington, U.S.A. & Linked $\mathrm{Th}$ and $\mathrm{Tm}$ & 1050100 & 5.5 & $\begin{array}{l}\text { Bending (1983), St. Marie and } \\
\text { Kesler (2000) }\end{array}$ \\
\hline 18 & Anjireh-Vejin mines, Iran & Linked Th and salinity & 1160000 & 8.3 & Lisenbee and Uzunlar (1988) \\
\hline \multicolumn{6}{|c|}{ Non-typical MVT } \\
\hline A & $\begin{array}{l}\text { Barite district, Washington County, } \\
\text { Southeast Missouri, U.S.A. }\end{array}$ & $\begin{array}{l}\text { Unlinked } \mathrm{Th} \text { and } \mathrm{Tm} \text {, } \\
\text { Ranges of } \mathrm{Th} \text { and salinity }\end{array}$ & & & $\begin{array}{l}\text { Kaiser et al. (1987), Leach } \\
(1980)\end{array}$ \\
\hline \multirow{3}{*}{$\begin{array}{l}\text { B } \\
\text { C } \\
\text { D }\end{array}$} & $\begin{array}{l}\text { Sweetwater district, East Tennessee, U.S.A. } \\
\text { (F-Ba- } \mathrm{Zn}-\mathrm{Pb} \text { deposit) }\end{array}$ & Ranges of $\mathrm{Th}$ and $\mathrm{Tm}$ & & & Misra et al. (1989) \\
\hline & English Pennines, England (F-Pb-Zn-Ba deposits) & Ranges of Th and salinity & & & Rogers $(1977,1978)$ \\
\hline & $\begin{array}{l}\text { Fluorspar district, Illinois-Kentucky, U.S.A. } \\
\text { (F-Zn-Pb deposit) }\end{array}$ & Linked $\mathrm{Th}$ and salinity & & & Richardson and Pinckney (1984) \\
\hline
\end{tabular}

O histograma para fluidos pré-mineralização compila dados de apenas cinco depósitos (Figura 1.9) e mostra uma flutuação de temperaturas de $50^{\circ} \mathrm{C}$ a $140^{\circ} \mathrm{C}$. Segundo os autores, há, aparentemente, três modas, com contribuições distintas de depósitos particulares, sendo a primeira delas em $120^{\circ} \mathrm{C}$ a $140^{\circ} \mathrm{C}$, a segunda em $100^{\circ} \mathrm{C}$ a $110^{\circ} \mathrm{C}$ e a terceira em $60^{\circ} \mathrm{C}$ a $70^{\circ}$ C.
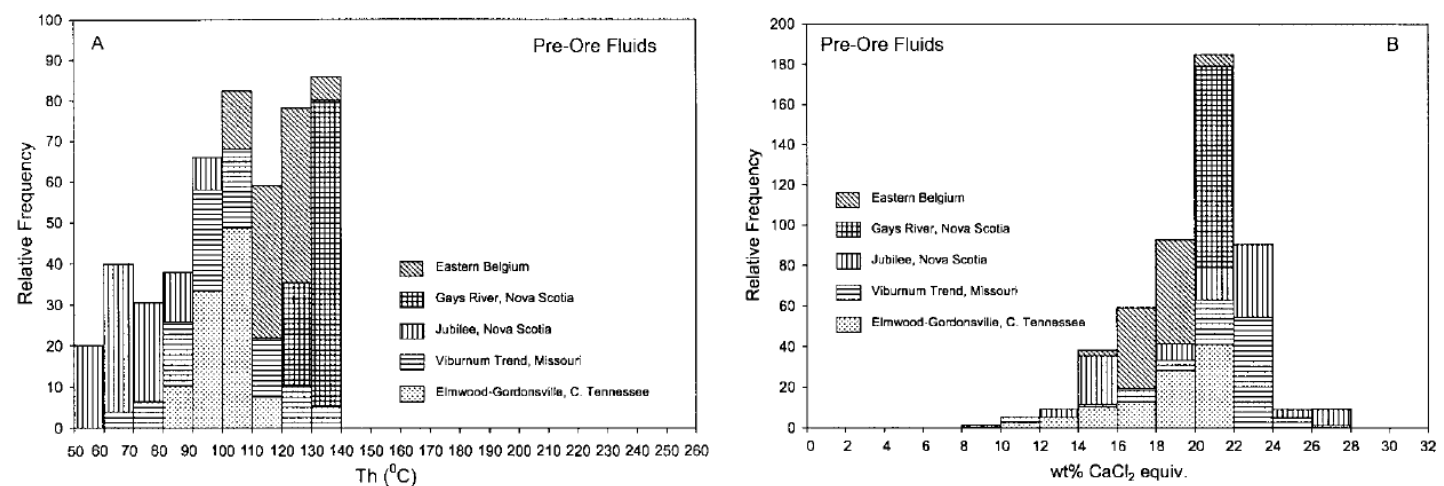

Figura 1.9. Histogramas de temperatura de homogeneização (A) e salinidade (B) de fluidos pré-mineralização para vários depósitos MVT (Basuki \& Spooner, 2002).

A natureza polimodal dos histogramas de temperatura (Figura 1.9-A) é devido tanto à variabilidade entre e dentre as regiões. Em claro contraste, as salinidades do fluido do estágio pré-mineralização (Figura 1.9-B) são caracterizadas por uma moda bem definida entre $20 \% \mathrm{e}$ $22 \%$ em peso de $\mathrm{CaCl}_{2}$ equivalente, com uma flutuação dos valores entre $8 \%$ e $28 \%$ em peso de $\mathrm{CaCl}_{2}$ equivalente (Basuki \& Spooner, 2002). 
Para os fluidos associados ao estágio mineralizante (Figura 1.10), as temperaturas apresentam variabilidade maior, com valores entre $60^{\circ} \mathrm{C}$ e $260^{\circ} \mathrm{C}$, com inclinação positiva distinta, e, para os autores, os dados apontam uma moda principal clara entre $110^{\circ} \mathrm{C}$ e $120^{\circ} \mathrm{C}$, e uma moda menos bem definida entre $140^{\circ} \mathrm{C}$ e $160^{\circ} \mathrm{C}$. Dados de salinidade exibem valores entre $4 \%$ a $32 \%$ em peso de $\mathrm{CaCl}_{2}$ equiv., com uma moda bem desenvolvida entre $20 \%$ a $24 \%$ em peso de $\mathrm{CaCl}_{2}$ equiv. (Basuki \& Spooner 2002).
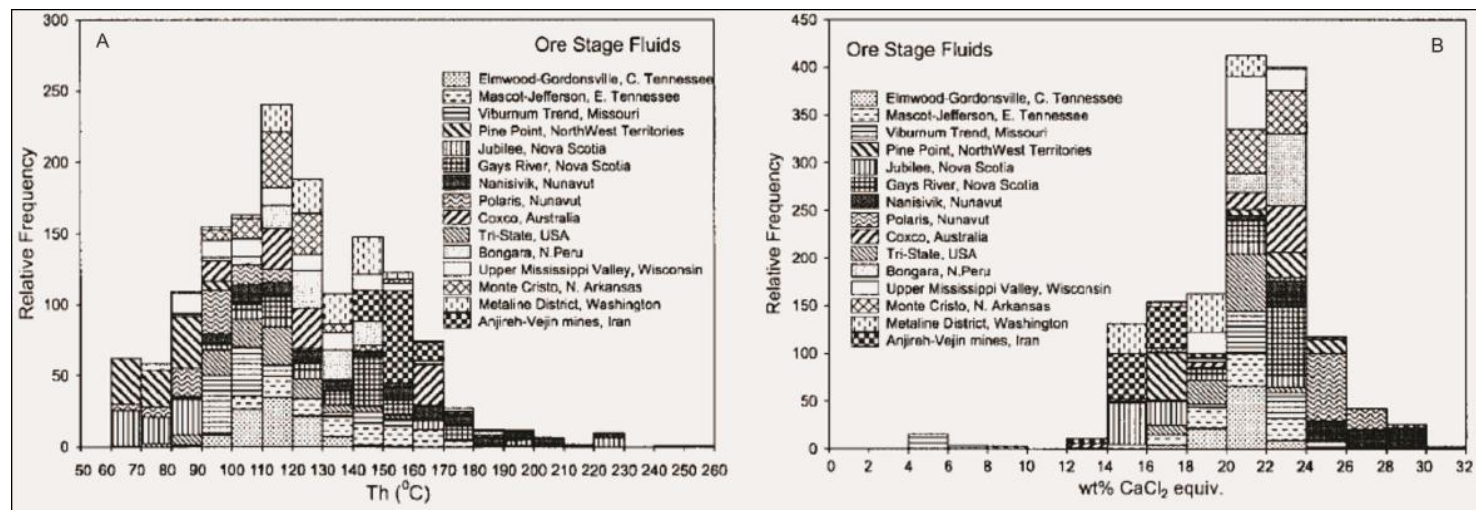

Figura 1.10. Histogramas de temperatura de homogeneização (A) e salinidade (B) de fluidos do estágio mineralizante para vários depósitos MVT (Basuki \& Spooner, 2002).

Temperaturas de fluidos do estágio pós-mineralização (Figura 1.11-A) exibem valores que variam entre $60^{\circ} \mathrm{C}$ e $260^{\circ} \mathrm{C}$ com salinidades (Figura 1.11 -B) entre $2 \%$ a $32 \%$ em peso de $\mathrm{CaCl}_{2}$ equiv. Para Basuki \& Spooner (2002), nota-se três modas para temperatura e salinidade. A moda mais significativa para temperatura é de $110^{\circ} \mathrm{C}$ a $120^{\circ} \mathrm{C}$; temperaturas entre $80^{\circ} \mathrm{C} \mathrm{e}$ $100^{\circ} \mathrm{C}$ e entre $140^{\circ} \mathrm{C}$ e $150^{\circ} \mathrm{C}$ definem as modas adicionais. A moda principal para salinidade ocorre entre $20 \%$ e $22 \%$ em peso de $\mathrm{CaCl}_{2}$ equiv.; as outras duas modas ocorrem entre $12 \%$ a $14 \%$ e entre $26 \%$ a $28 \%$ em peso de $\mathrm{CaCl}_{2}$ equiv. e os autores ressaltam que as modas de baixa temperatura e de baixa salinidade ocorrem somente nos dados do estágio de pós-mineralização.
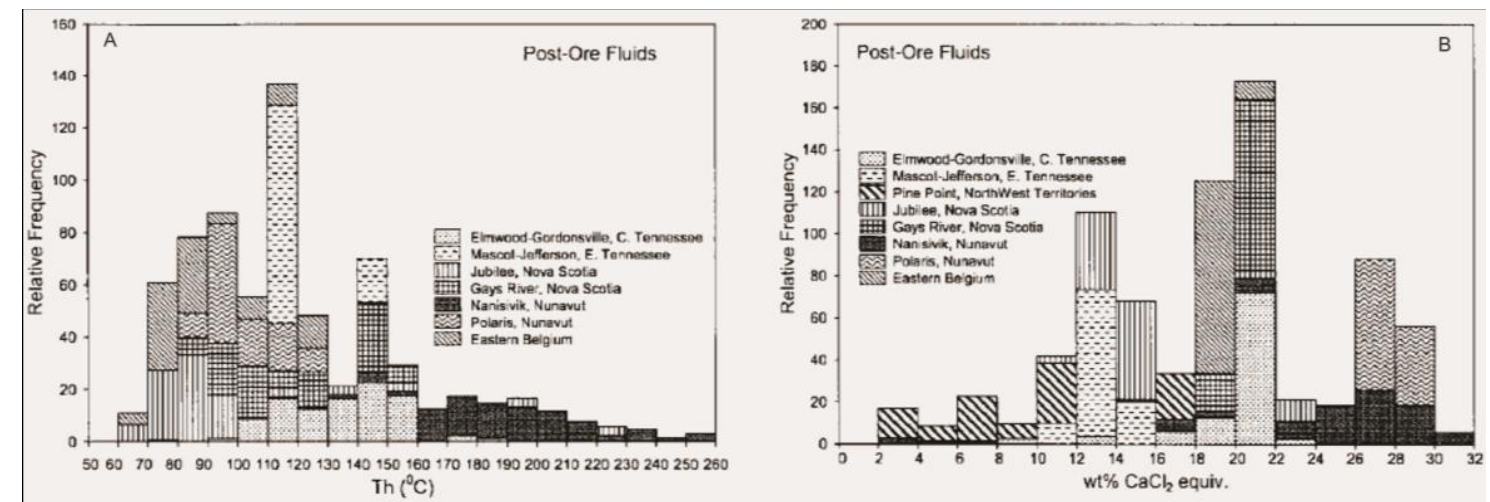

Figura 1.11. Histogramas de temperatura de homogeneização (A) e salinidade (B) de fluidos pós-mineralização para vários depósitos MVT (Basuki \& Spooner, 2002).

A interpretação dos dados dessa compilação deve ser cautelosa, uma vez que ocorrem diferentes processos físico-químicos e até biológicos durante a atividade hidrotermal, assim como diferentes mecanismos de precipitação de minério. 


\subsubsection{Inclusões fluidas em depósitos tipo Irish}

Os depósitos hospedados em calcários dos campos de minério irlandeses representam uma das maiores concentrações de metais básicos na crosta rasa. Diferentemente dos depósitos MVT, o estudo de inclusões fluidas neste tipo de depósito são recentes e esparsos, desta maneira, informações, as quais forneceriam restrições em relação a modelos conflitantes, são escassas (Wilkinson, 2001).

Inclusões fluidas foram estudadas principalmente em dolomita, calcita, barita, quartzo e esfalerita. As inclusões fluidas são relativamente simples, compreendendo bifásicas aquosas compostas por fases líquida + vapor ( $<10 \%$ de vapor compõe o volume), e inclusões monofásicas (Wilkinson, 2001).

De acordo com Wilkinson (2001), três grandes grupos de inclusões fluidas foram distinguidos: (i) de salinidade moderada (10\% a $15 \%$ em peso de $\mathrm{NaCl}$ equiv.), $\mathrm{T}_{\mathrm{h}}$ moderada $\left(150^{\circ} \mathrm{C}\right.$ a $\left.220^{\circ} \mathrm{C}\right)$, cujas a primeira fusão irrompe em torno de $-22^{\circ} \mathrm{C}$ que indica a predominância de $\mathrm{NaCl}$; (ii) de alta salinidade (20\% a 25\% em peso de $\mathrm{NaCl}$ equiv.) e baixa $\mathrm{T}_{\mathrm{h}}$ $\left(<150^{\circ} \mathrm{C}\right)$, com primeira fusão em torno de $-55^{\circ} \mathrm{C}$ que indica sistema $\mathrm{H}_{2} \mathrm{O}-\mathrm{NaCl}-\mathrm{CaCl}_{2}$; (iii) uma população subordinada de baixa salinidade ( $<5 \%$ em peso de $\mathrm{NaCl}$ equiv.), baixa a moderada $\mathrm{T}_{\mathrm{h}}\left(120^{\circ} \mathrm{C}\right.$ a $\left.200^{\circ} \mathrm{C}\right)$ e de composição salina dominantemente representada por $\mathrm{NaCl}$. Em geral, aceita-se a hipótese de que a deposição de minério ocorreu durante a mistura dos fluidos do tipo (i) com tipo (ii). Considera-se que o fluido (i) carreou o conteúdo metalífero e um conteúdo de enxofre "hidrotermal" para os depósitos (Everett et al., 1999), enquanto inferese que o fluido (ii) era enriquecido em $\mathrm{H}_{2} \mathrm{~S}$ produzido bacteriologicamente (Wilkinson et al., 1998).

Por vezes observa-se a formação de clatrato que é indicativo de um sistema que contem $\mathrm{CO}_{2}$, cuja presença tem um papel importante, pois indica aumento da acidez do fluido mineralizante por meio de hidrólise durante resfriamento e ascensão. Processos de dissolução e substituição por fluidos de baixo $\mathrm{pH}$ são de fundamental importância para a gênese de depósitos do tipo Irish e hidrólise de $\mathrm{CO}_{2}$ fornece o mecanismo pelo qual tais fluidos ácidos seriam gerados, de acordo com Large (1981).

Em geral, a média das temperaturas para este tipo de depósito está entre $180^{\circ} \mathrm{C}$ e $240^{\circ} \mathrm{C}$. Estas temperaturas são consideravelmente mais altas que as relacionadas a depósitos MVT e modelos de fluxo de salmouras bacinais de baixa temperatura são inapropriados (Wilkinson, 2001).

\subsubsection{Inclusões fluidas no Grupo Vazante: depósitos de Morro Agudo, Fagundes, Ambrósia e Vazante}

\subsubsection{Morro Agudo}

As rochas hospedeiras da mineralização do depósito de Morro Agudo são dolarenitos e brechas intraformacionais que pertencem à Formação Morro do Calcário do Grupo Vazante segundo Cunha (1999). Segundo esta autora a mineralização é interceptada ao leste por um sistema de falhas normais de direção N-S, e a partir desta falha a mina foi subdivida (Figura 1.12) em três blocos ("A", "B" e "C") e os corpos de minério foram individualizados ("G", "H", "I", "J", "K", “L”, "M” e "N"), mas geralmente são agrupados em corpos "GHI", "JKL", "M" e "N" de acordo com Romagna \& Costa (1988).

Cunha (1999) realizou estudos de inclusões fluidas em inclusões primárias e pseudosecundárias (cogenéticas) em esfalerita do depósito de Morro Agudo e identificou dois tipos de 
inclusões, no que diz respeito ao conteúdo de fluido em temperatura ambiente: (i) bifásicas $(\mathrm{L}+\mathrm{V})$, com volume de vapor entre $5 \%$ a $20 \%$ e (ii) inclusões monofásicas. A autora individualizou os histogramas da microtermometria de acordo com os diferentes corpos de minério a fim de procurar zoneamentos termais verticais e horizontais.

Definiu-se o modelo composicional para inclusões como o sistema $\mathrm{NaCl}-\mathrm{H}_{2} \mathrm{O}$ e temperaturas do eutético abaixo de $-21,2^{\circ} \mathrm{C}$ foram descartadas. Foi relatada dificuldade em distinguir se os cristais que estavam sofrendo fusão eram gelo ou hidrohalita, devido ao reduzido tamanho das inclusões e, devido a isso, as temperaturas de fusão foram calculadas separadamente, usando temperaturas de fusão do gelo e da hidrohalita. Segundo a autora, a homogeneização nos ensaios de aquecimento ocorreu sempre para a fase líquida. Os valores de temperatura mínima de aprisionamento $\left(\mathrm{T}_{\mathrm{h}}\right)$ das inclusões indicam um decréscimo dos valores destes à medida que se afasta da zona de falha. Os dados de salinidade e temperatura mínima de aprisionamento são resumidos nas figuras 1.13 e 1.14 .

Como relatado por Cunha et al., (2000) as inclusões fluidas estudadas indicam fluidos de salinidade moderada a baixa; zonação de temperaturas de homogeneização indicam uma diminuição lateral do gradiente termal, com maiores temperaturas próximas a zona de falha e estão de acordo com dados isotópicos de pares cogenéticos de esfalerita e galena de Misi et al., (1996). Aqueles autores sugerem que fluidos mais salinos próximos ao sítio de maior descarga sofreram mistura com fluidos bacinais de salinidades baixas a moderadas, que resultou em uma diminuição das salinidades à medida que se afasta da zona de falha. Esses resultados corroboram com a hipótese de que a zona de falha que limita o depósito a leste pode ter atuado como uma zona alimentadora de fluidos mineralizantes e reforça a importância do controle estrutural para este depósito.

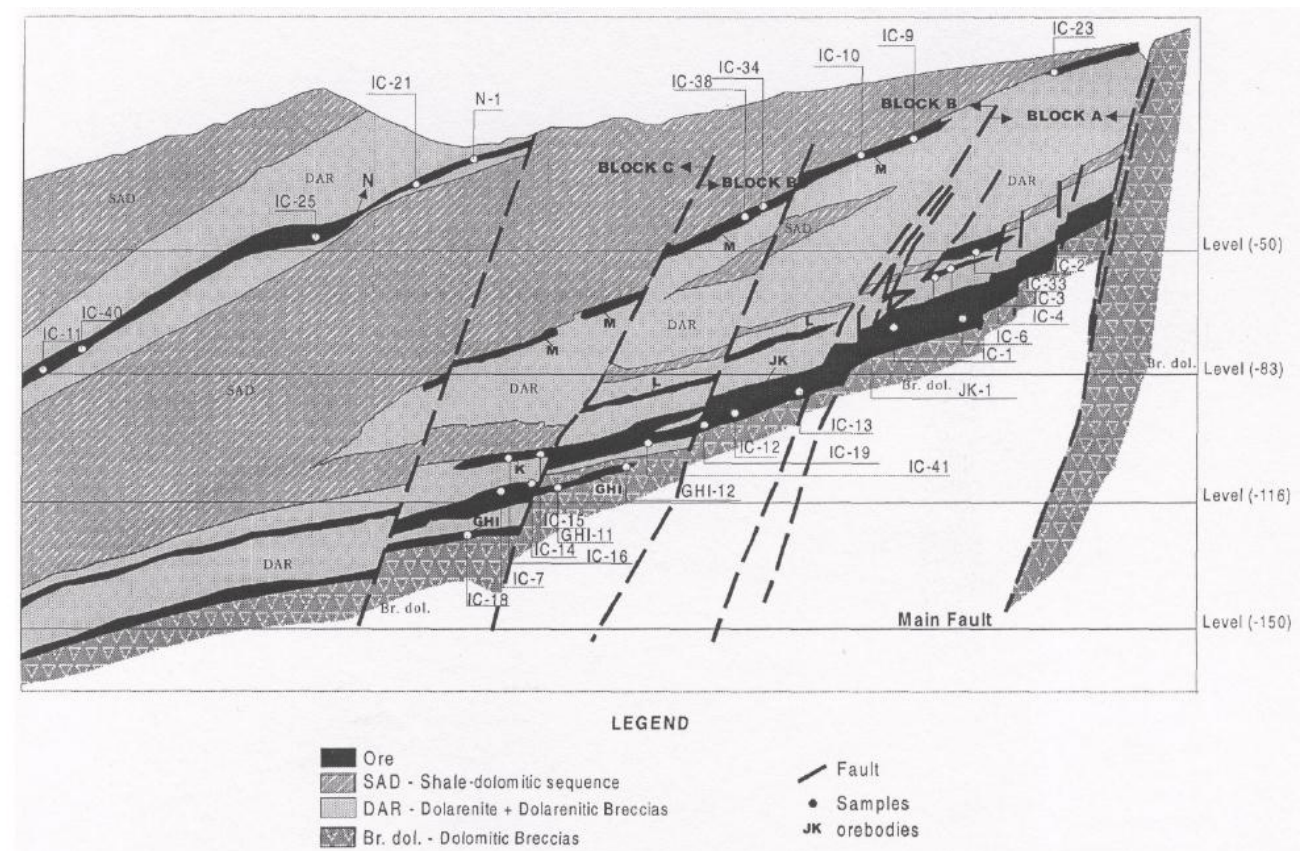

Figura 1.12. Localização dos sítios de amostragem ao longo de seção leste-oeste, linha 74 da mina subterrânea de Morro Agudo. Fonte: relatório interno CMM (Cunha, 1999). 


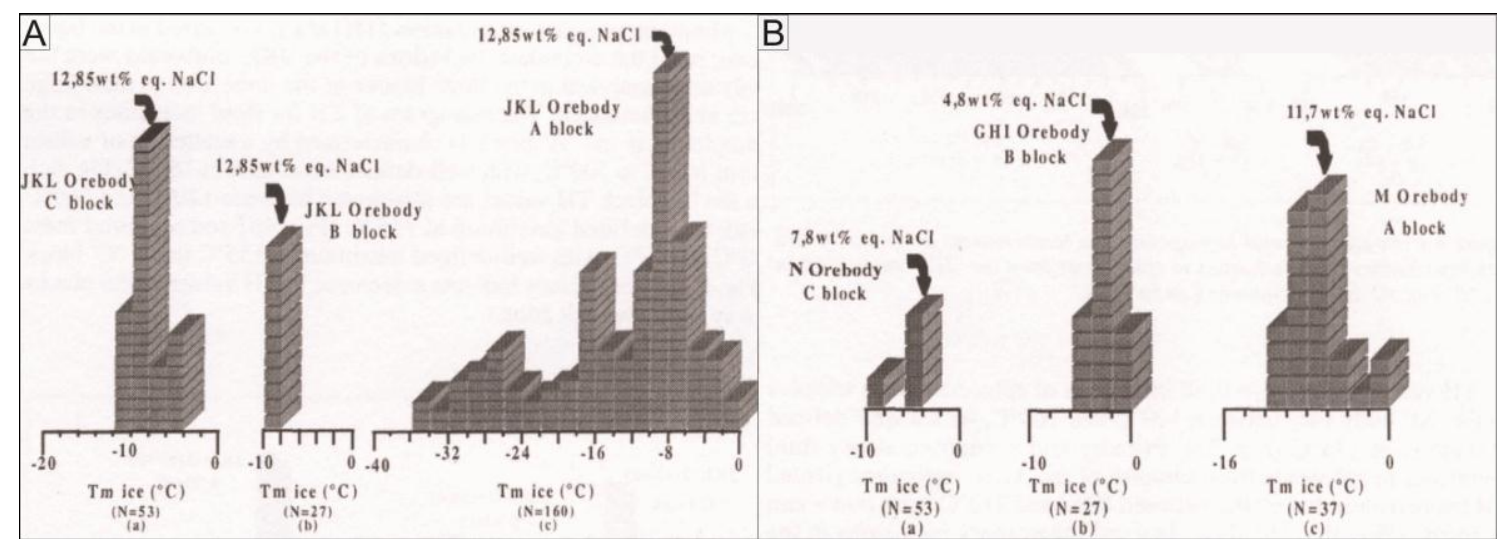

Figura 1.13. A: Histograma das temperaturas de fusão de gelo para inclusões fluidas primárias e pseudo-secundárias em esfaleritas dos corpos "JKL" (blocos "C", "B" e "A"). B: Histograma das temperaturas de fusão de gelo para inclusões fluidas primárias e pseudo-secundárias em esfaleritas de: (a) corpo "N" - bloco "C"; (b) corpo "GHI" bloco "B"; e (c) corpo "M" - bloco "A" (extraído de Cunha et al., 2000). As salinidades apresentadas para as modas mais representativas dos histogramas foram calculadas para fusão do gelo ao invés de fusão da hidrohalita.

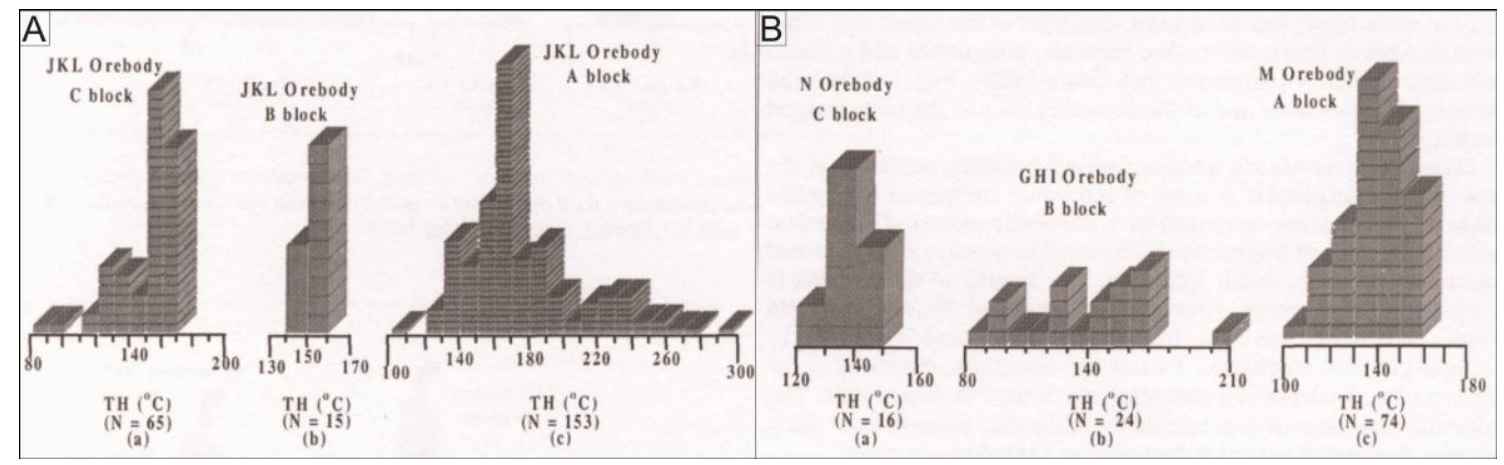

Figura 1.14. A: Histograma das temperaturas de homogeneização total $\left(T_{h}\right)$ para inclusões fluidas primárias e pseudosecundárias em esfaleritas do corpo "JKL" nos blocos "C", "B" e "A". B: Histograma das temperaturas de homogeneização total $\left(\mathrm{T}_{\mathrm{h}}\right)$ para inclusões fluidas em esfaleritas de: (a) corpo "N" - bloco "C"; (b) corpo "GHI" bloco "B"; e (c) corpo "M" - bloco "A" (extraído de Cunha et al., 2000).

\subsubsection{Fagundes}

O Depósito de Fagundes está hospedado na sequência carbonática com filitos subordinados do Membro Pamplona Superior, da Formação Morro do Calcário, Grupo Vazante. Monteiro (2002) realizou um estudo sistemático das inclusões fluidas em minerais de minério e de ganga no depósito de Fagundes. Esta autora sugere a presença de cátions divalentes no sistema composicional salino das inclusões fluidas estudadas devido aos baixos valores de temperatura de eutético $\left(\mathrm{T}_{\mathrm{e}}\right)$. Os dados resultantes deste estudo são sumarizados na Tabela 1.2.

Neste depósito observa-se a diminuição dos valores de $T_{e}$ em relação à sucessão paragenética e Monteiro (2002) reforça que os valores mais baixos de temperatura de fusão de gelo e de temperatura do eutético caracterizam, de forma geral, os fluidos associados ao estágio tardio de mineralização, o que indica que durante a evolução da alteração hidrotermal houve uma tendência de aumento da salinidade e diminuição da $T_{e}$. Isso sugere uma transição da composição dos sistemas salinos como evidenciado na Figura 1.15. Esta tendência de evolução dos fluidos pode ser indicativa de processo de mistura de um fluido inicial menos salino, contendo $\mathrm{MgCl}_{2}$, com soluções mais salinas enriquecidas em $\mathrm{CaCl}_{2}$, semelhante a que ocorre em depósitos MVT clássicos e Irish, incluindo-se os depósitos de Gays River e Jubilee, Canadá, nos quais o estágio principal de mineralização tem sido relacionado ao aumento das razões de $\mathrm{CaCl}_{2} / \mathrm{NaCl}$ (Monteiro, 2002). Outras evidências de mistura de fluidos observadas por esta autora seriam variações de temperaturas de fusão de gelo $\left(\mathrm{T}_{\mathrm{fg}}\right)$ em inclusões fluidas primárias de 
um dado mineral, assim como variações de $\mathrm{T}_{\mathrm{e}}$ em um mesmo mineral e $\mathrm{T}_{\mathrm{fg}}$ baixas (em torno de $-1,8^{\circ} \mathrm{C}$ a $\left.-0,5^{\circ} \mathrm{C}\right)$ em inclusões secundárias que sugerem mistura posterior com fluidos meteóricos.

Tabela 1.2. Sumário dos dados microtermométricos do depósito de Fagundes. $\mathrm{P}=$ primária; $\mathrm{PS}=$ pseudo-secundária; S = secundária (extraído de Monteiro, 2002).

\begin{tabular}{|c|c|c|c|c|c|c|c|}
\hline Estágio & Mineral & IF & & TE ( $\left.{ }^{\circ} \mathrm{C}\right)$ & Tfg $\left({ }^{\circ} \mathrm{C}\right)$ & $\left.\mathrm{TH}{ }^{\circ} \mathrm{C}\right)$ & $\begin{array}{c}\text { Salinidade } \\
\text { (\% em poso de } \\
\mathrm{NaCl} \text { equiv.) }\end{array}$ \\
\hline \multicolumn{8}{|l|}{ FAGUNDES } \\
\hline $\begin{array}{l}\text { Pré - } \\
\text { mineralizaçäo }\end{array}$ & Quartzo (l) & I & $\mathrm{P}$ & $-46,0 a-35,2$ & $-10,2 a-3,7$ & 143,5 a 190,6 & 5,9 a 14,2 \\
\hline \multirow{4}{*}{$\begin{array}{l}\text { Sin - } \\
\text { mineralizaçäo }\end{array}$} & \multirow{2}{*}{$\begin{array}{l}\text { Esfalerita } \\
\text { zonada (ii) }\end{array}$} & I & $P$ & $-51,7$ a $-39,0$ & $-8,1$ a $-3,6$ & 138,9 a 260,2 & 5,8 a 11,8 \\
\hline & & ॥ & $S$ & $-53,4$ a $-39,4$ & $-9,5 a-2,5$ & 91,1 a 117,5 & 4,1 a 13,4 \\
\hline & \multirow[t]{2}{*}{ Quartzo (II) } & I & $P$ & $-44,9 a-32,8$ & $-6,0$ a $-4,3$ & 148,0 a 204,9 & 6,8 a 9,2 \\
\hline & & II & $\mathrm{S}$ & $-41,8 a-33,8$ & $-9,8 a-5,9$ & 149,9 a 186,6 & 9,1 a 13,7 \\
\hline \multirow[t]{7}{*}{ Mobilizaçāo } & \multirow[t]{3}{*}{ Esfalerita (III) } & lb & $\mathrm{P}$ & $-51,0 \mathrm{a}-44,2$ & $-6,2 a-3,2$ & 127,8 a 264,7 & 5,2 a 9,5 \\
\hline & & llb & $S$ & $-55,4 a-44,9$ & $-2,9 a-0,5$ & \multirow{2}{*}{97,1 a 197,2} & 0,8 a 4,7 \\
\hline & & IIIb & $S$ & $-49,8 a-44,2$ & $-4,3 a-0,5$ & & 0,0 a 6,8 \\
\hline & Dolomito & 1 & $P$ & $-57,2 a-46,7$ & $-11,6 a-6,3$ & 165,1 a 189,0 & 9,6 a 15,6 \\
\hline & \multirow[t]{2}{*}{ (VII) } & II & $S$ & $-52,9 a-41,9$ & $-11,0 a-7,9$ & 157,0 a 184,0 & 11,6 a 15,0 \\
\hline & & III & $S$ & $-54,7 \mathrm{a}-42,4$ & $-9,8 a-1,8$ & 157,0 a 182,0 & 3,0 a 13,7 \\
\hline & Quartzo (III) & & $S$ & $-54,4 a-43,8$ & $-11,7 a-6,4$ & & 9,7 a 15,7 \\
\hline $\begin{array}{l}\text { Mineralização } \\
\text { tardia }\end{array}$ & Esfalerita (IV) & & $S$ & $-52,3$ a $-40,3$ & $-8,9 a-4,0$ & & 6,4 a 12,7 \\
\hline
\end{tabular}

Como descrito por Monteiro (2002), pode-se observar também uma correlação entre os valores de $T_{h}$ e $T_{f g}$, em que os valores mais altos de $T_{h}$ (aproximadamente $200^{\circ}$ C) estão associados aos menores valores de $\mathrm{T}_{\mathrm{fg}}$, ou seja, a maiores salinidades, embora essa correlação seja invertida para inclusões fluidas em esfalerita, onde maiores valores de $\mathrm{T}_{\mathrm{h}}$ (até $264^{\circ} \mathrm{C}$ ) estão associados a salinidades moderadas (média de $9 \%$ em peso de $\mathrm{NaCl}$ equiv.) e valores de $\mathrm{T}_{\mathrm{h}}$ menores $\left(\right.$ como $130^{\circ} \mathrm{C}$ ) correspondem a salinidades mais altas (até $12 \%$ em peso de $\mathrm{NaCl}$ equiv.).

Nas inclusões de esfalerita do Depósito de Fagundes não é observada, de modo claro, um trend de variação contínua de $\mathrm{T}_{\mathrm{h}} \mathrm{e} \mathrm{T}_{\mathrm{fg}}$ (Figura 1.16), que poderia indicar com maior segurança a presença de processos de mistura de fluidos, embora estes possam ser sugeridos pelas variações nas salinidades (Monteiro, 2002). De acordo com Shepherd et al., (1985) e Wilkinson (2001), trends no espaço $\mathrm{T}_{\mathrm{h}}$-salinidade semelhantes ao observado são indicativos de mistura isotermal de fluidos (Monteiro, 2002). 


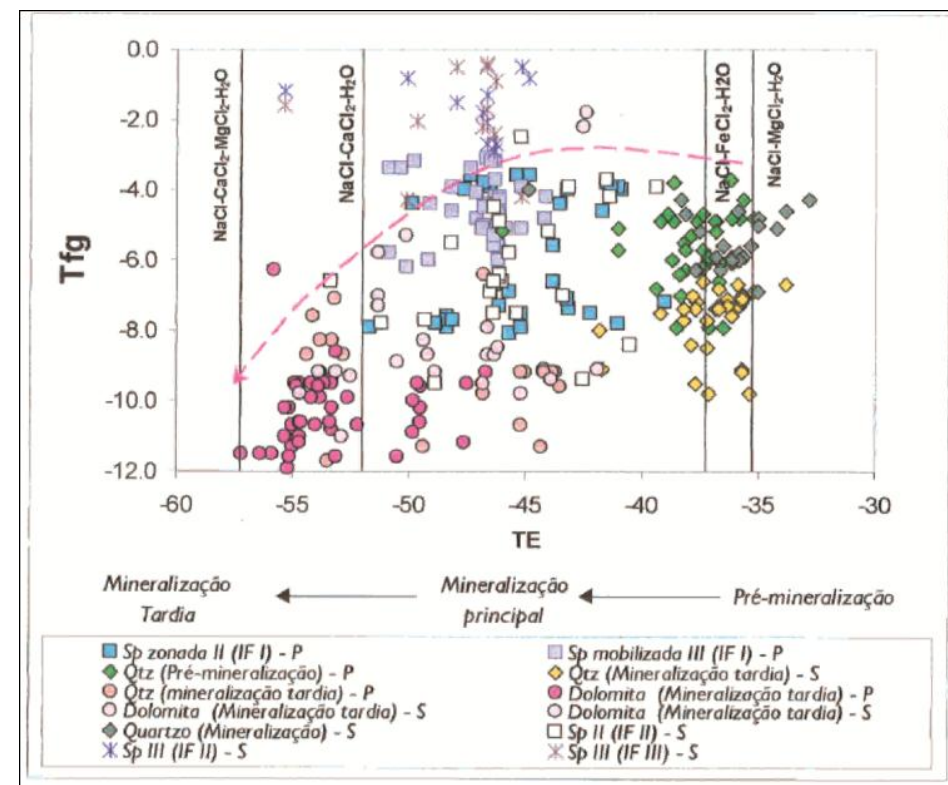

Figura 1.15. Diagrama bivariante mostrando as relações entre as temperaturas de eutético $\left(\mathrm{T}_{\mathrm{e}}\right)$ e de fusão do gelo $\left(\mathrm{T}_{\mathrm{fg}}\right)$ em diferentes gerações de inclusões fluidas do depósito de Fagundes. As temperaturas do eutético para diferentes sistemas são de Davis et al., (1990) e Borisenko (1978). Abreviações: Sp = esfalerita; Qtz = quartzo; Dol = dolomita; $\mathrm{IF}=$ inclusões fluidas; $\mathrm{P}$ = primárias; $\mathrm{S}=$ secundárias (extraído de Monteiro, 2002).

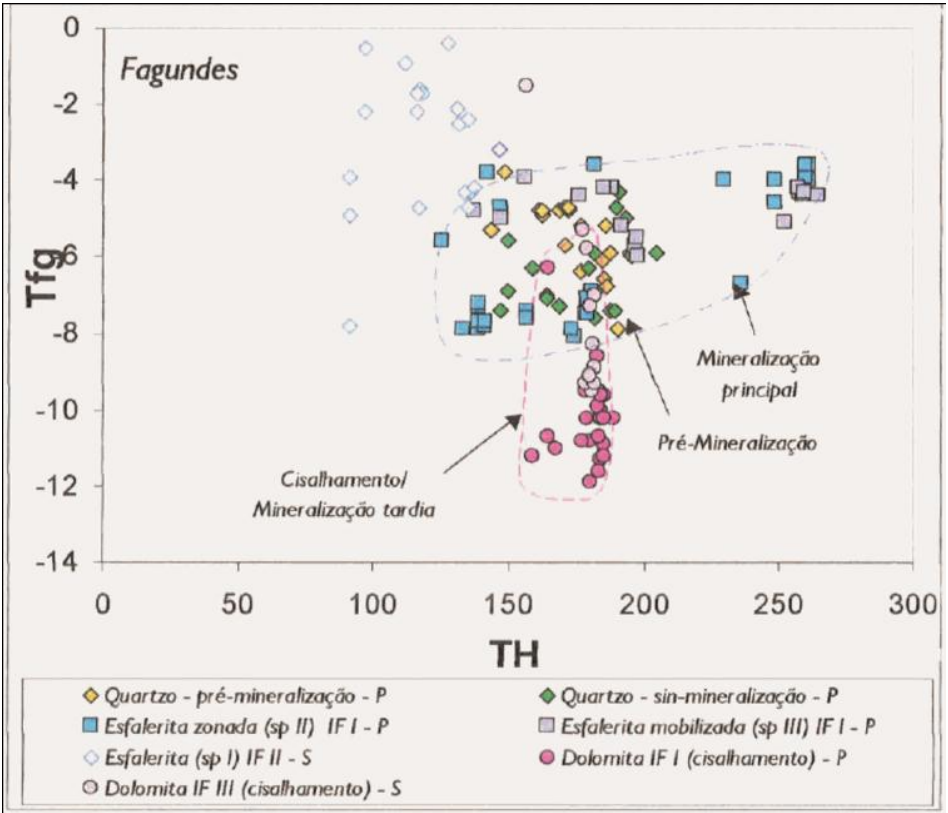

Figura 1.16. Diagrama mostrando a variação de $T_{h}$ e $T_{\text {fg }}$ em inclusões fluidas em diferentes fases do depósito de Fagundes. Abreviações: $\mathrm{IF}=$ inclusão fluida; $\mathrm{P}=$ primária; $\mathrm{S}=$ secundária (extraído de Monteiro, 2002).

\subsubsection{Ambrósia}

O Depósito de Ambrósia está hospedado na sequência carbonática com filitos subordinados do Membro Pamplona Superior, da Formação Morro do Calcário, Grupo Vazante. Estudos de inclusões fluidas no depósito de Ambrósia foram realizados por Monteiro (2002) em minerais de minério e de ganga. Esta autora relata que as $\mathrm{T}_{\mathrm{e}}$ estão dentro de um intervalo similar de temperatura (aproximadamente entre $55,6^{\circ} \mathrm{C}$ e $--40,6^{\circ} \mathrm{C}$ ) exceto por inclusões fluidas em esfalerita tardia, em que o intervalo de temperaturas é mais baixo $\left(-65,1^{\circ} \mathrm{C}\right.$ a $\left.-61,0^{\circ} \mathrm{C}\right)$. Segundo Davis et al., (1990) apud Monteiro (2002), entretanto, estes valores estão dentro do intervalo de $\mathrm{T}_{\mathrm{e}}$ metaestável do sistema $\mathrm{H}_{2} \mathrm{O}-\mathrm{NaCl}-\mathrm{CaCL}_{2}$. A síntese dos estudos de inclusões fluidas do depósito de Ambrósia está disposta na Tabela 1.3. 
Tabela 1.3. Sumário dos dados microtermométricos do depósito de Ambrósia. $\mathrm{P}=$ primária; $\mathrm{PS}=$ pseudo-secundária; $\mathrm{S}=$ secundária (extraído de Monteiro, 2002).

\begin{tabular}{|c|c|c|c|c|c|c|c|}
\hline Estágio & Mineral & IF & & TE ("C) & $\mathrm{Tfg}\left({ }^{\circ} \mathrm{C}\right)$ & $T H\left({ }^{\circ} \mathrm{C}\right)$ & $\begin{array}{c}\text { Salinidade } \\
\text { (\% em peso de } \\
\mathrm{NaCl} \text { equiv.) }\end{array}$ \\
\hline \multicolumn{8}{|l|}{ AMBROSSIA } \\
\hline \multirow{3}{*}{$\begin{array}{l}\text { Sin - } \\
\text { mineralização }\end{array}$} & Esfalerita (l) & 1 & $P$ & $-55,6 a-48,5$ & $-17,0 a-10,6$ & 167,8 a 281,6 & 14,6 a 20,2 \\
\hline & & II & $S$ & $-55,6$ a $-49,8$ & $-4,0 a-16,5$ & 106,4 a 140,9 & 6,4 a 19,8 \\
\hline & & III & $\mathrm{S}$ & $-55,4 a-53,5$ & $-22,8 a-21,9$ & 131,0 a 136,5 & $>23,1$ \\
\hline \multirow[t]{3}{*}{ Mobilizaçáo } & Quartzo & 1 & $\mathrm{P}$ & $-53,6 a-46,5$ & $-19,4 a-12,8$ & 241,6 a 243,5 & 16,7 a 21,9 \\
\hline & & ॥ & $S$ & $-55,4 a-40,6$ & $-8,4 a-3,2$ & 123,6 a 137,9 & 5,2 a 12,2 \\
\hline & & III & $\mathrm{S}$ & $-52,8$ a $-43,9$ & $-4,3 a-0,3$ & 114,7 a 124,8 & 0,5 a 6,8 \\
\hline $\begin{array}{l}\text { Mineralização } \\
\text { tardia }\end{array}$ & Esfalerita (III) & 1 & $P$ & $-65,1$ a $-61,0$ & $-21,3 a-12,8$ & 105,2 a 136,2 & $16,7 \mathrm{a} \sim 23,1$ \\
\hline
\end{tabular}

De acordo com Monteiro (2002), a salinidade dos fluidos mineralizantes em Ambrósia era mais elevada (até $21,9 \%$ em peso de $\mathrm{NaCl}$ equiv.) do que em Fagundes e destacou a participação de fluidos tardios mais salinos, apesar de haverem inclusões secundárias caracterizadas por baixas salinidades também.

Em Ambrósia, grupos de inclusões primárias em quartzo e esfalerita (I) indicaram temperaturas entre $241,6^{\circ}$ e $243,5^{\circ} \mathrm{C}$ e entre $167,8^{\circ}$ e $281,6^{\circ} \mathrm{C}$, respectivamente (Monteiro, 2002). Temperaturas mais baixas foram verificadas tanto nas inclusões secundárias em quartzo $\left(115^{\circ}\right.$ a $\left.138^{\circ} \mathrm{C}\right)$ e esfalerita (I) $\left(106,0^{\circ}\right.$ a $\left.140,9^{\circ} \mathrm{C}\right)$, como em inclusões primárias na esfalerita tardia (III), que apresentaram valores de $\mathrm{T}_{\mathrm{h}}$ entre $105,2^{\circ}$ e $136,2^{\circ} \mathrm{C}$ (Monteiro, 2002).

Em Ambrósia, esfalerita tardia exibe uma grande dispersão de valores de $T_{\mathrm{fg}}$, que também sugere mistura de fluidos, assim como em Fagundes, segundo Monteiro (2002). Esta autora também relatou que inclusões fluidas secundárias apresentam $T_{h}$ dentro do intervalo de variação das $T_{h}$ de inclusões fluidas primárias, mas com variação da salinidade, que pode sugerir contribuição de fluidos meteóricos, menos salinos, de modo análogo ao depósito de Fagundes, embora ocorram inclusões fluidas secundárias em esfalerita do estágio principal de mineralização com altos valores de salinidade que indicam participação de fluidos salinos na mineralização tardia (Figura 1.17). 


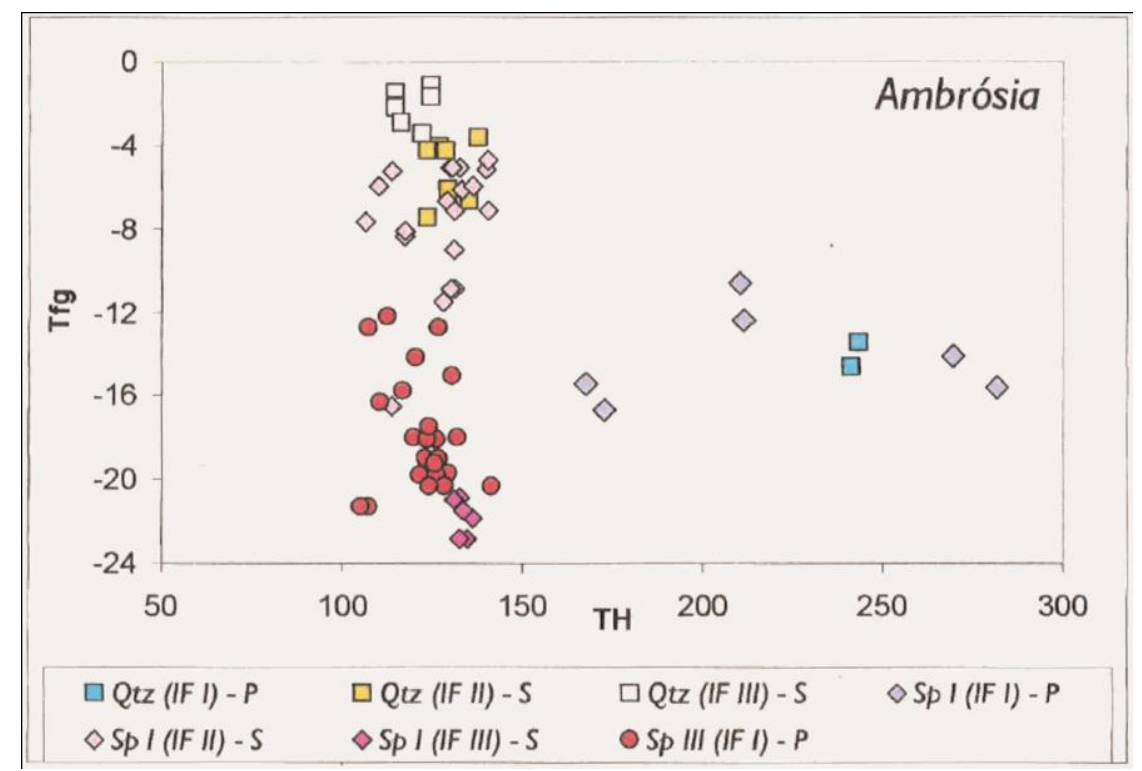

Figura 1.17. Diagrama que mostra a variação de $T_{h}$ (temperatura de homogenizacão) e $T_{f g}$ (temperatura de fusão do gelo) em inclusões fluidas em diferentes fases do depósito de Ambrósia (extraído de Monteiro, 2002).

\subsubsection{Vazante}

O Depósito de Vazante está hospedado na sequência carbonática com filitos subordinados do Membro Pamplona Inferior, da Formação Serra do Poço Verde, Grupo Vazante. O minério encontra-se hospedado em brechas hidrotermais associadas à Zona de Falha Vazante. Monteiro (2002) também levou a cabo o estudo de inclusões fluidas no depósito de Vazante. No trabalho desta autora, foi relatado $T_{e}$ dentro de um intervalo de temperatura bastante semelhante ao relatado no depósito de Fagundes (entre $-53,8^{\circ} \mathrm{C}$ a $-41,3^{\circ} \mathrm{C}$ ). A síntese dos resultados dos minerais nos quais as inclusões foram estudadas está disposta na Tabela 1.4.

Tabela 1.4. Sumário dos dados microtermométricos do depósito de Vazante. $\mathrm{P}=$ primária; $\mathrm{OS}=$ pseudo-secundária; $\mathrm{S}$ = secundária (extraído de Monteiro, 2002).

\begin{tabular}{|c|c|c|c|c|c|c|c|}
\hline Estágio & Mineral & IF & & TE ( $\left.{ }^{\circ} \mathrm{C}\right)$ & $T \mathrm{fg}\left({ }^{\circ} \mathrm{C}\right)$ & TH( $\left.{ }^{\circ} \mathrm{C}\right)$ & $\begin{array}{c}\text { Salinidade } \\
\text { (\% em peso de } \\
\mathrm{NaCl} \text { equiv.) }\end{array}$ \\
\hline \multicolumn{8}{|l|}{ VAZANTE } \\
\hline \multirow[t]{3}{*}{ Mineralização } & Esfalerita & 1 & $P$ & $-53,8 a-41,3$ & $-23,9 a-11,3$ & 170,0 a 232,1 & $15,3 a>23,0$ \\
\hline & & III & $S$ & $-53,5$ a 52,3 & $-11,0 a-7,0$ & 100,5 a 165,0 & 10,5 a 15,0 \\
\hline & & IV & s & & & $\sim 50$ & \\
\hline \multirow{6}{*}{$\begin{array}{l}\text { Pós } \\
\text { mineralização } \\
\text { (Brechas } \\
\text { "cockade") }\end{array}$} & Quartzo & la & $\mathbf{P}$ & $-47,4 a-37,0$ & $-13,0 a-8,6$ & 140,0 a 160,0 & 12,4 a 16,9 \\
\hline & & $\mathrm{lb}$ & $P$ & $-47,2 a-38,9$ & $-10,8 a-4,2$ & 135,0 a 160,0 & 6,7 a 14,8 \\
\hline & & Ic & $P$ & $-52,2$ a $-37,9$ & $-12,9 a-9,0$ & 140,0 a 160,0 & 12,9 a 16,8 \\
\hline & & Id & PS & $-47,4 a-39,9$ & $-14,2$ a $-9,1$ & 140,0 a 160,0 & 13,0 a 18,0 \\
\hline & & \| & S & $-54,5$ a $-42,8$ & $-17,3 \mathrm{a}-12,2$ & 85,0 a 140,0 & 16,1 a 20,4 \\
\hline & & III & S & $-53,5$ a $-36,6$ & $-14,2 \mathrm{a}-4,1$ & 75,0 a 90,0 & 6,5 a 18,0 \\
\hline
\end{tabular}

Temperaturas de fusão do gelo em inclusões fluidas primárias em esfalerita exibem valores baixos que conferem salinidades elevadas (15,3\% a >23,0\% em peso de $\mathrm{NaCl}$ equiv.) em relação ao depósito de Fagundes. Quartzo tardio a mineralização exibe valores de salinidade predominantemente entre $12,4 \%$ e $17,9 \%$, em peso de $\mathrm{NaCl}$ equiv., embora ocorra um grupo de inclusões primárias com salinidades menores, entre $6,7 \%$ a $14,8 \%$ em peso de $\mathrm{NaCl}$ equiv., de acordo com Monteiro (2002). As inclusões secundárias em quartzo definem duas faixas de 
variação de salinidade entre $6,5 \%$ e $17,9 \%$ em peso de $\mathrm{NaCl}$ equiv. e $16,1 \%$ e $20,4 \%$ em peso de $\mathrm{NaCl}$ equiv.

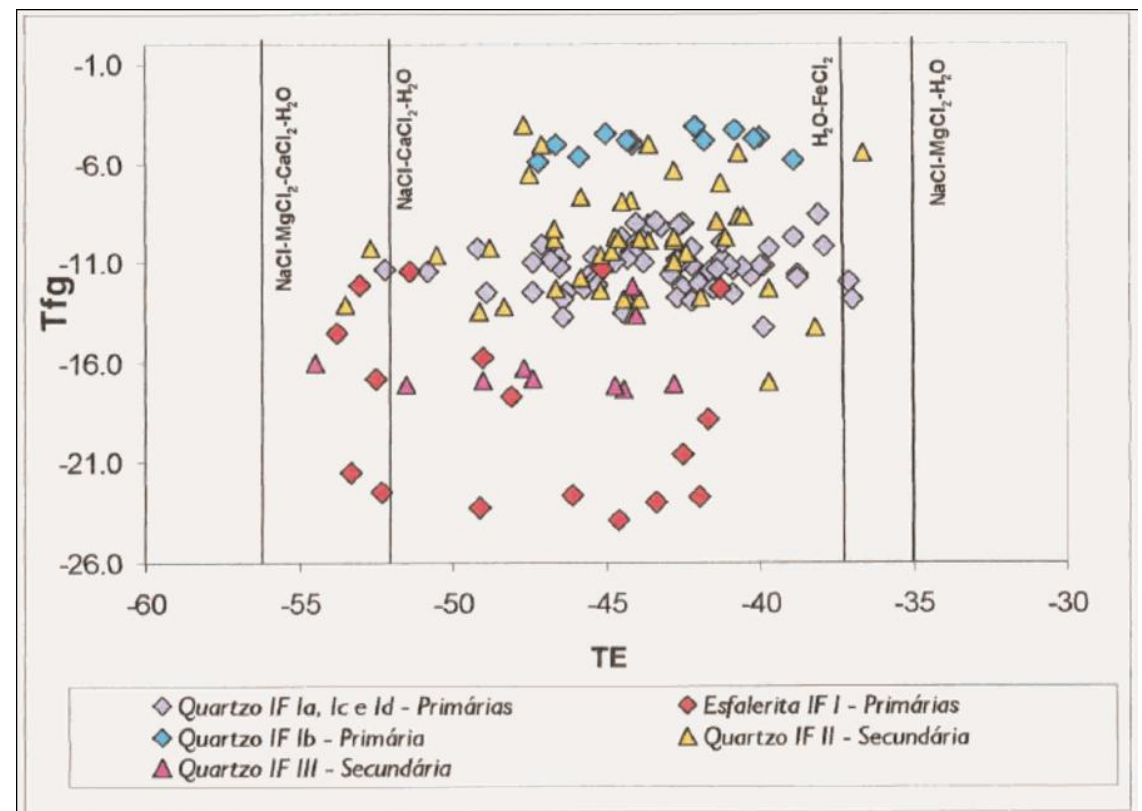

Figura 1.18. Variações das temperaturas de eutético $\left(\mathrm{T}_{\mathrm{e}}\right)$ e de fusão do gelo $\left(\mathrm{T}_{\mathrm{fg}}\right)$ em diferentes gerações de inclusões fluidas do Depósito de Vazante. Temperaturas de eutético para diferentes sistemas de Borisenko (1978) e Davis et al., (1990) (extraído de Monteiro, 2002).

As variações de salinidade entre a esfalerita associada à zona de Falha de Vazante (valores de salinidade mais altos), quartzo associado à brecha posterior ao estágio de mineralização willemítica (salinidades intermediárias) e inclusões secundárias dividas em dois grupos (um de altos valores de salinidade e outro com baixos valores de salinidade) em conjunto com o fato de também ocorrerem variações nas medidas de $\mathrm{T}_{\mathrm{e}}$ indicam mistura de fluidos, de acordo com Monteiro (2002), com indícios de participação de fluidos meteóricos posteriores a mineralização sulfetada (Figura 1.18).

Segundo Monteiro (2002), o intervalo de temperatura para inclusões fluidas primárias em quartzo varia está entre $140^{\circ} \mathrm{C}$ e $160^{\circ} \mathrm{C}$ e entre $75^{\circ} \mathrm{C}$ a $90^{\circ} \mathrm{C}$ para inclusões secundárias. $\mathrm{O}$ intervalo de valores de $\mathrm{T}_{\mathrm{h}}$ para inclusões fluidas primárias em esfalerita concentra-se entre $201^{\circ}$ $\mathrm{C}$ e $232^{\circ} \mathrm{C}$ e entre $100,5^{\circ} \mathrm{C}$ a $165,0^{\circ} \mathrm{C}$ para inclusões fluidas secundárias nesta mesma fase mineral.

O diagrama $T_{h} \times T_{f g}$ (Figura 1.19) mostra, assim como os outros depósitos da Faixa Vazante - Paracatu, variação de $T_{\mathrm{fg}}$ em relativamente pequeno intervalo de variação de $T_{h}$ em quartzo tardio à mineralização, que pode indicar participação de fluidos meteóricos nos estágios tardios do sistema hidrotermal, de acordo com Monteiro (2002). 


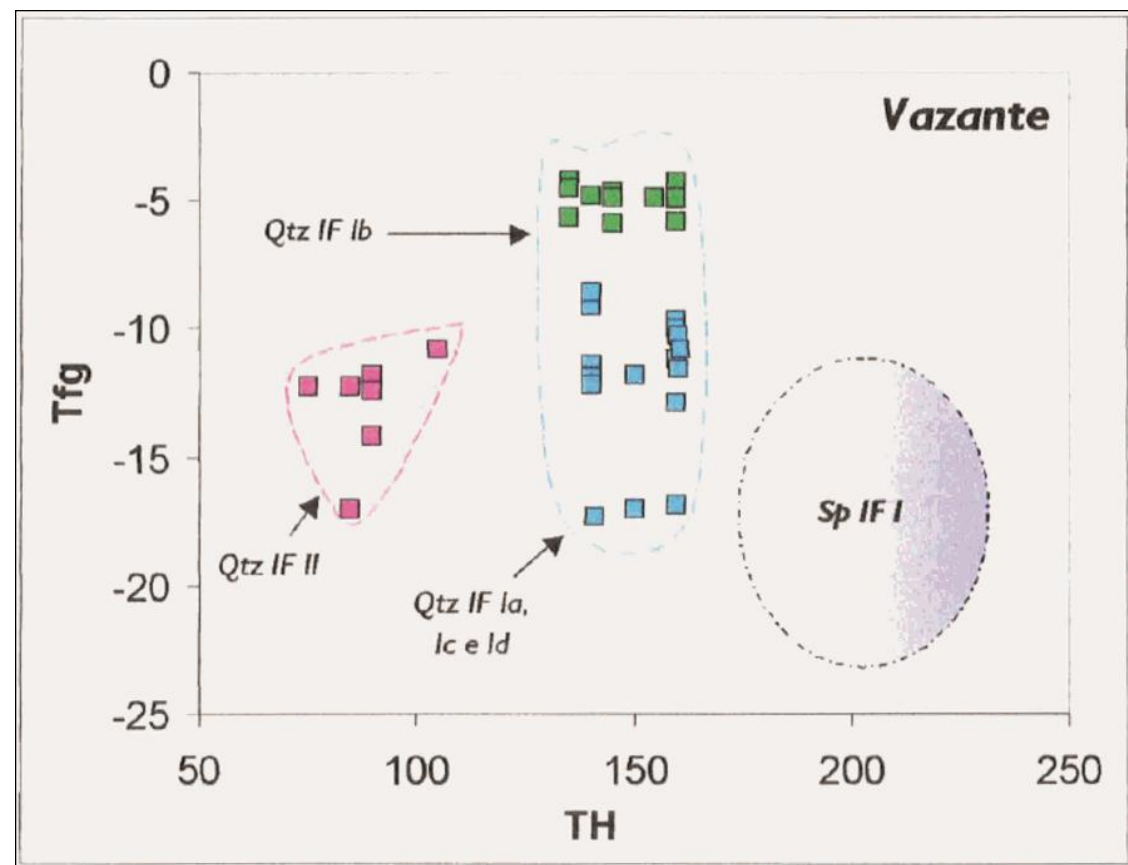

Figura 1.19. Variações dos valores de $\mathrm{T}_{\mathrm{h}}$ e $\mathrm{T}_{\mathrm{fg}}$ em inclusões fluidas em quartzo do depósito de Vazante (extraído de Monteiro, 2002).

\subsubsection{Discussões da revisão teórica}

A comparação dos dados de $\mathrm{T}_{\mathrm{e}} \mathrm{e} \mathrm{T}_{\mathrm{fg}}$ para inclusões primárias para os depósitos de Fagundes, Ambrósia e Vazante (Figura 1.20) mostra que os fluidos relacionados à mineralização primária de Ambrósia, considerada epigenética, são mais salinos que aqueles responsáveis pela mineralização inicial de Fagundes, tardi-diagenética, mas apresentam intervalos de $\mathrm{T}_{\mathrm{e}}$ e $\mathrm{T}_{\mathrm{fg}}$ semelhantes aos fluidos associados com a mineralização tardia de Fagundes (Monteiro, 2002).

Essas características podem indicar semelhança entre os fluidos hidrotermais envolvidos nos estágios de mineralização epigenética nos dois depósitos, embora em Ambrósia o envolvimento de fluidos com alta salinidade nos estágios finais de mineralização seja ainda mais importante (Monteiro, 2002). De acordo com Monteiro (2002), fluidos salinos estão associados à mineralização sulfetada no Depósito de Vazante, no entanto o grande espalhamento dos valores de $\mathrm{T}_{\mathrm{e}}$ e $\mathrm{T}_{\mathrm{fg}}$ não permita uma avaliação a respeito da evolução desses fluidos (Figura 1.20).

De acordo com Monteiro (2002) a composição predominante do sistema salino é $\mathrm{H}_{2} \mathrm{O}$ $\mathrm{NaCl}-\mathrm{CaCl}_{2}$, para os três depósitos e segundo Goldstein \& Reynolds (1994) e Wilkinson (2001), esse sistema salino representa a composição de salmouras bacinais em termos de constituintes maiores. Fluidos com composições próximas a esse sistema são também produzidos por interação fluido-rocha durante alteração retrógrada de silicatos associada ao retrometamorfismo (Oberthür et al., 2000).

Algumas variações composicionais podem ser inferidas para os diferentes estágios de mineralização, como fluidos com a presença de $\mathrm{MgCl}_{2}$ (e possivelmente $\mathrm{FeCl}_{2}$ ) em inclusões fluidas da fase-mineralização em Fagundes, possivelmente relacionados à extensiva formação de dolomita baroque que ocorre neste depósito, e que antecedeu a mineralização, de acordo com Monteiro (2002). O estágio mineralizante é caracterizado por fluidos mais ricos em $\mathrm{Ca}^{2+}$, semelhante ao descrito para outros depósitos hospedados em sequências carbonáticas, em que o 
estágio principal de mineralização é marcado por um incremento nas razões $\mathrm{CaCl}_{2} / \mathrm{NaCl}$, possivelmente devido à dolomitização.

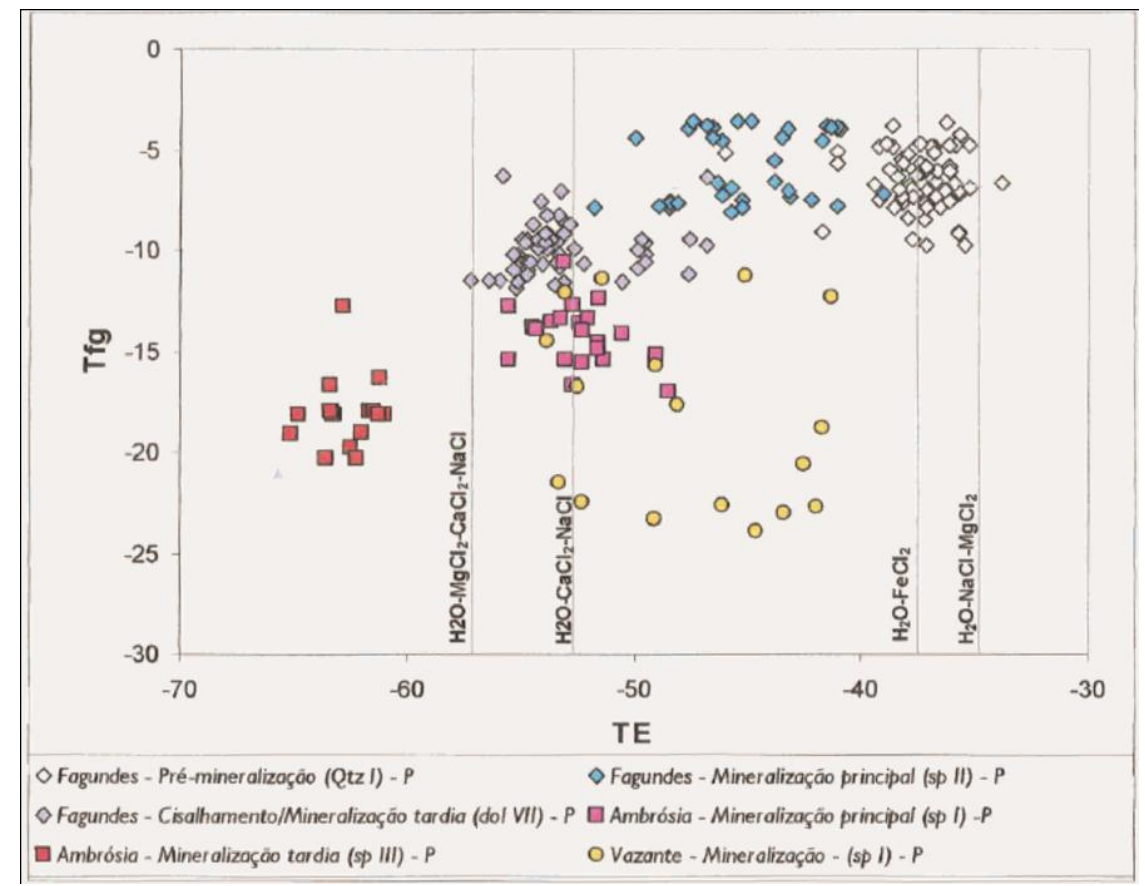

Figura 1.20. Variação de $\mathrm{T}_{\mathrm{e}}$ e $\mathrm{T}_{\mathrm{fg}}$ em inclusões fluidas dos depósitos de Vazante, Ambrósia e Fagundes. Temperaturas de eutético para diferentes sistemas de Davis et al., (1990) e Borisenko (1978). Abreviações: $\mathrm{sp} \equiv+$ esfalerita; Qtz = quartzo; dol = dolomita; $\mathrm{P}$ = primária (extraído de Monteiro, 2002).

Monteiro (2002) relata que mistura de fluidos ao longo do tempo podem ter sido importantes nos depósitos de Fagundes, Ambrósia e Vazante, a partir da comparação de valores de $\mathrm{T}_{\mathrm{h}}$ e $\mathrm{T}_{\mathrm{fg}}$ das inclusões primárias (Figura 1.21), e destaca que, regionalmente, pode-se diferenciar três tipos de fluidos hidrotermais:

- Fluido de temperatura anormalmente alta $\left(>200^{\circ} \mathrm{C}\right)$, com maiores valores de $\mathrm{T}_{\mathrm{e}}$ em relação aos demais tipos de fluidos, salinidade moderada a baixa, considerado metalífero, com importante papel nos estágios iniciais de mineralização;

- Fluido de temperaturas moderadas $\left(190^{\circ} \mathrm{C}\right.$ a $\left.140^{\circ} \mathrm{C}\right)$, salinidade elevada e composição próxima a do sistema $\mathrm{H}_{2} \mathrm{O}-\mathrm{NaCl}-\mathrm{CaCl}_{2}$, que pode ter tido importante papel nos episódios de mineralização tardia, epigenética;

- Fluido hidrotermal, de temperaturas moderadas a baixas $\left(90^{\circ} \mathrm{C}\right.$ a $\left.160^{\circ} \mathrm{C}\right)$, com baixa salinidade, possivelmente com contribuição meteórica (Monteiro, 2002).

Segundo Monteiro (2002), as altas temperaturas medidas nos depósitos de Vazante, Fagundes e Ambrósia não se assemelham as descritas para depósitos MVT, mas sim a depósitos do tipo Irish, e defende que as temperaturas mais altas podem refletir o ambiente de formação, diferente de áreas cratônicas estáveis características dos depósitos MVT.

As mineralizações de zinco com chumbo subordinado da Faixa Vazante - Paracatu também podem estar relacionadas a um ambiente geotectônico instável, vinculado aos primeiros fronts cavalgamento da Faixa Brasília, o que explicaria a sobreposição de episódios de mineralização tanto em estágios diagenéticos, como deformacionais, durante o Evento Brasiliano (Monteiro, 2002). 


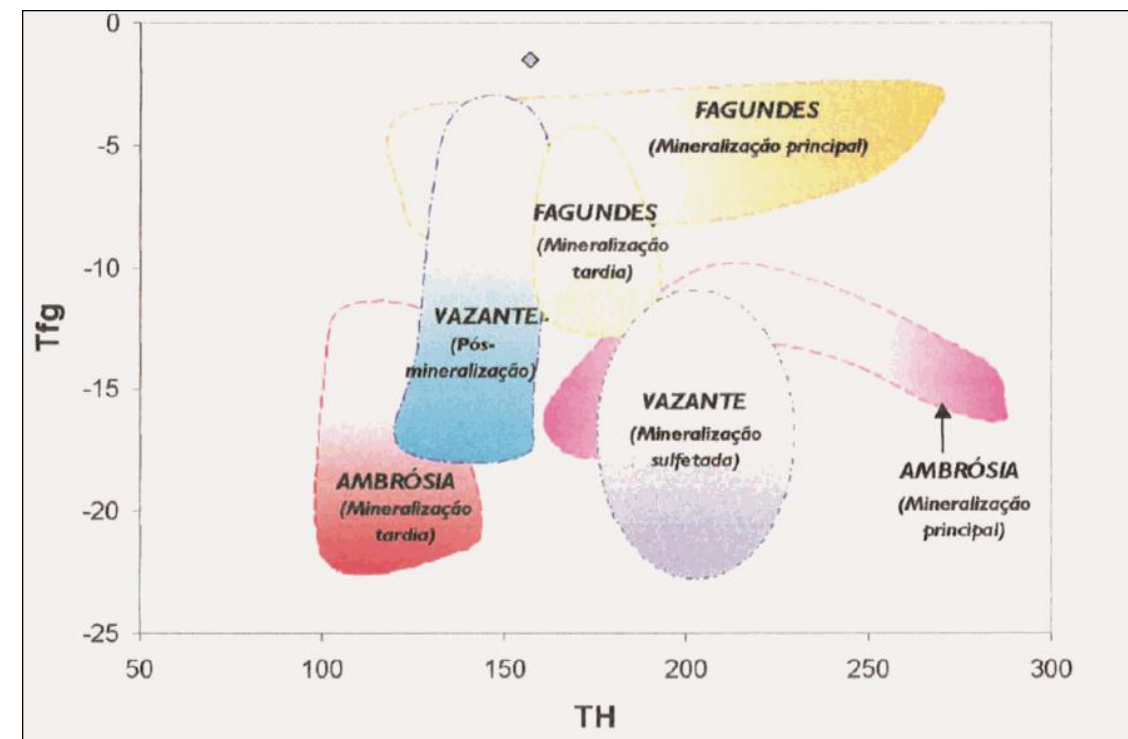

Figura 1.21. Variações dos valores de $\mathrm{T}_{\mathrm{h}}$ e $\mathrm{T}_{\mathrm{fg}}$ em inclusões fluidas dos depósitos de Vazante, Ambrósia e Fagundes (extraído de Monteiro, 2002).

A distribuição das medidas no diagrama $\mathrm{T}_{\mathrm{h}} \times \mathrm{T}_{\mathrm{fg}}$ dos quatro depósitos de $\mathrm{Zn} \pm \mathrm{Pb}$ da Faixa Vazante - Paracatu indicam algumas semelhanças (Figura 1.22), como a grande concentração de medidas em intervalos intermediários de $\mathrm{T}_{\mathrm{fg}}(-4 \%$ a $-10 \%$ em peso de $\mathrm{NaCl}$ equiv.) e de $\mathrm{T}_{\mathrm{h}}\left(130^{\circ} \mathrm{C}\right.$ a $\left.190^{\circ} \mathrm{C}\right)$.

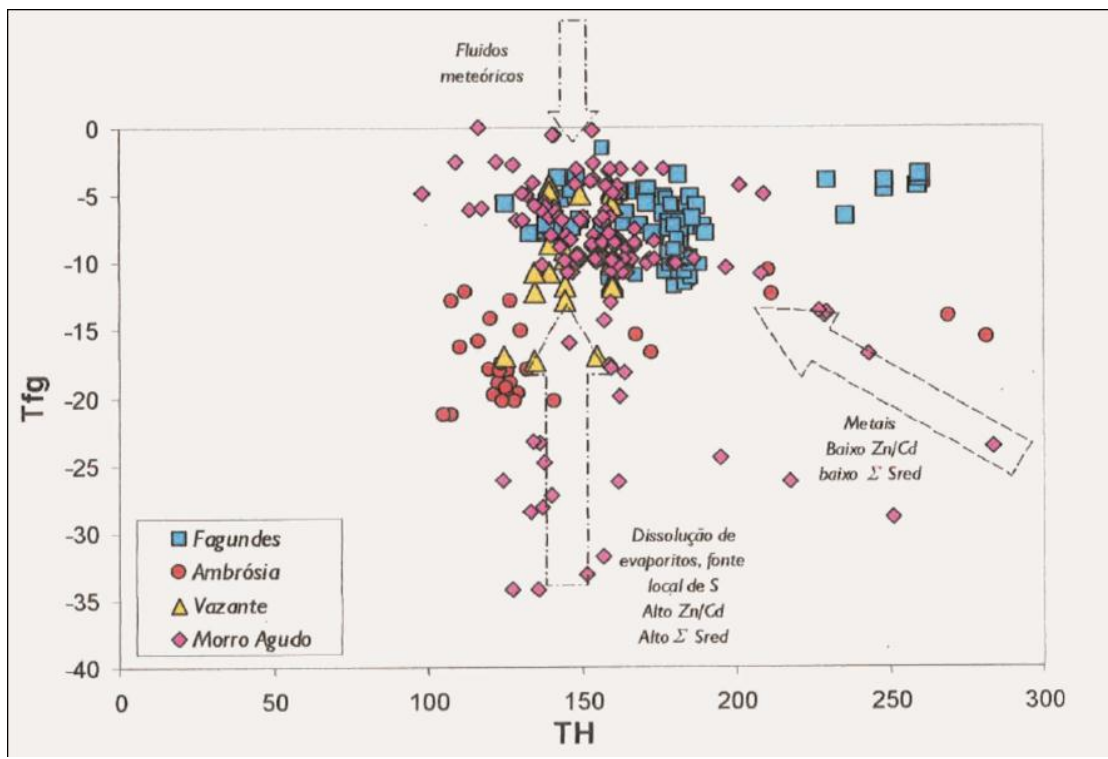

Figura 1.22. Comparação entre os dados de Th e Tfg em inclusões fluidas primárias dos depósitos de Fagundes, Ambrósia, Vazante e Morro Agudo. Dados de Morro Agudo foram extraídos de Cunha (1999) (extraído de Monteiro ,2002).

Aparentemente há uma variabilidade considerável de salinidades e temperaturas de fluidos que revelam um amplo espectro de depósitos que transpassam os limites de categorias de mineralização. $\mathrm{O}$ estudo de inclusões fluidas tem um importante papel na racionalização do entendimento das mineralizações de metais básicos em tais ambientes (Wilkinson, 2001). 


\subsection{MATERIAIS E MÉTODOS}

Um testemunho de sondagem representativo (VZVG003: de 48,25 a 620,96 m) com várias feições pós-sedimentares e vários estágios e estilos de alteração hidrotermal e duas zonas mineralizadas foi selecionado para estudo detalhado. Esse testemunho foi descrito em colaboração com a equipe de exploração da Votorantim Metais S.A.. Cento e trinta e uma amostras foram selecionadas para esse estudo, incluindo feições de interação fluido-rocha desde os episódios hidrotermais mais precoces aos mais tardios. Amostras de rocha inalterada também foram amostradas para comparação. Um subgrupo de quarenta e seis amostras com relevantes feições hidrotermais e de relações de corte foram selecionadas para estudos petrográficos e mineralógicos de detalhe. Dessas amostras, nove foram selecionadas para estudo de inclusões fluidas, embora somente em cinco amostras foram identificadas inclusões fluidas que pudessem ser trabalhadas.

Para as descrições petrográficas, foram utilizados os microscópios petrográficos binoculares de luz transmitida e refletida Zeiss Image A2m e Olympus BX-60, com camêras fotográficas acopladas AxioCamICc3 e Q-Imaging 5.0 RTV, respectivamente, no Laboratório de Petrografia do Instituto de Geociências da Universidade de Brasília.

Análises de química mineral foram realizadas em dolomita, willemita, esfalerita, pirita, galena, calcocita, covelita e greenockite. $\mathrm{O}$ equipamento utilizado foi a microssonda eletrônica modelo JEOL JXA 8900RL do Laboratório de Microscopia e Microanálises do Instituto de Geociências da Universidade de Brasília. As análises foram realizada sob $15 \mathrm{kV}$ de tensão, 20 $\mathrm{nA}$ de corrente e feixe de $1 \mu \mathrm{m}$ de diâmetro. As medições foram realizadas por 10 segundos no pico do elemento e 5 segundos nos backgrounds inferior e superior. Os Padrões e limites de detecção para os elementos analisados em microssonda eletrônica estão listados na tabela 1.5.

Tabela 1.5. Padrões e limites de detecção para os elementos analisados em microssonda eletrônica. Detec. = detecção; Elem. $=$ elemento; Lim. $=$ limite.

\begin{tabular}{|l|l|l|l|l|l|}
\hline Elem. & Padrão & Lim. Detec. & Elem. & Padrão & Lim. Detec. \\
\hline $\mathrm{Na}$ & Albita & $54 \mathrm{ppm}$ & $\mathrm{Mg}$ & Forsterita & $45 \mathrm{ppm}$ \\
\hline $\mathrm{F}$ & Topázio & $108 \mathrm{ppm}$ & $\mathrm{Al}$ & Microclínio & $239 \mathrm{ppm}$ \\
\hline $\mathrm{Si}$ & Microclínio & $51 \mathrm{ppm}$ & $\mathrm{K}$ & Microclínio & $56 \mathrm{ppm}$ \\
\hline $\mathrm{Cl}$ & Vanadinita $_{\mathrm{Ti}}$ & $45 \mathrm{ppm}$ & $\mathrm{Ca}$ & $\mathrm{Andradita}_{3}$ & $61 \mathrm{ppm}$ \\
\hline $\mathrm{Mn}$ & $\mathrm{MnTiO}_{3}$ & $387 \mathrm{ppm}$ & $\mathrm{Cr}$ & $\mathrm{Cr}_{2} \mathrm{O}_{3}$ & $199 \mathrm{ppm}$ \\
\hline $\mathrm{P}$ & Apatita & $130 \mathrm{ppm}$ & $\mathrm{Sr}$ & $\mathrm{SrSO}_{4}$ & $130 \mathrm{ppm}$ \\
\hline $\mathrm{V}$ & Vanadinita & $128 \mathrm{ppm}$ & $\mathrm{Ba}$ & $\mathrm{BaSO}_{4}$ & $199 \mathrm{ppm}$ \\
\hline $\mathrm{Zn}$ & $\mathrm{ZnS}$ & $79 \mathrm{ppm}$ & $\mathrm{Fe}$ & $\mathrm{Fayalita}$ & $105 \mathrm{ppm}$ \\
\hline $\mathrm{Ge}$ & $\mathrm{Ge}$ & $801 \mathrm{ppm}$ & $\mathrm{As}$ & $\mathrm{AsGa}$ & $163 \mathrm{ppm}$ \\
\hline $\mathrm{Cu}$ & $\mathrm{CuFeS}$ & $\mathrm{ppm}$ & $\mathrm{Ga}$ & $\mathrm{AsGa}$ & $93 \mathrm{ppm}$ \\
\hline $\mathrm{Bi}$ & $\mathrm{Bi}$ & $158 \mathrm{ppm}$ & $\mathrm{Cd}$ & $\mathrm{Cd}$ & $97 \mathrm{ppm}$ \\
\hline $\mathrm{S}$ & $\mathrm{Pirita}$ & $308 \mathrm{ppm}$ & $\mathrm{Au}$ & $\mathrm{Au}$ & $200 \mathrm{ppm}$ \\
\hline $\mathrm{Sb}$ & $\mathrm{Sb}_{2} \mathrm{~S}_{3}$ & $29 \mathrm{ppm}$ & $\mathrm{Ag}$ & $\mathrm{Ag}$ & $63 \mathrm{ppm}$ \\
\hline
\end{tabular}

Os ensaios de resfriamento e aquecimento de inclusões fluidas foram executados em uma platina Linkam THMSG 600, instalada em um microscópio binocular Olympus BX-50 com até 1000 X de magnificação e com camêra Micropublisher Q-Imaging 3.3 no Laboratório de Inclusões Fluidas do Instituto de Geociências da Univesidade de Brasília. Durante os ensaios de resfriamento, as temperaturas foram medidas sob uma taxa de $0,5^{\circ} \mathrm{C} / \mathrm{min}$, enquanto durante os ensaios de aquecimento as temperaturas foram medidas sob uma taxa de $3,0^{\circ} \mathrm{C} / \mathrm{min}$, utilizando o programa de controle do sistema Linksys 32.

Análises de isótopos estáveis foram conduzidas em várias gerações de dolomita. Os grãos selecionados foram pulverizados a partir dos tabletes de amostra para confecção das seções 
delgadas, em função de restringir as relações paragenéticas da amostra com a fase mineral. As amostras foram pulverizadas por meio de uma broca dentária de carbeto de tungstênio com revestimento de $\mathrm{Ni}$, de formato cônico e diâmetro mínimo de corte de $0,40 \mathrm{~mm}$. A extração de $\mathrm{CO}_{2}$ foi feita através de reação com $\mathrm{H}_{3} \mathrm{PO}_{4}$ a $72^{\circ} \mathrm{C}$, após limpeza do recipiente por fluxo de $\mathrm{He}$. Determinação de $\delta^{13} \mathrm{C}$ e $\delta^{18} \mathrm{O}$ em dolomita foi realizada no Laboratório de Estudos Geocronológicos, Geodinâmicos e Ambientais da Universidade de Brasília. Foi utilizado um Thermo Scientific Gas Bench II com sistema de preparação e introdução on-line. Esse sistema opera com um fluxo contínuo acoplado a um Thermo Scientific DELTA V Isotope Ratio espectrômetro de massa. A precisão é de $0.05 \%$ para $\delta^{13} \mathrm{C}$ e $0.10 \%$ para $\delta^{18} \mathrm{O}$ e os resultados são relatados como valores $\delta^{13} \mathrm{C}$ PDB (Pee Dee Belemnite) e $\delta^{18} \mathrm{O}$ PDB e $\delta^{18} \mathrm{O}$ SMOW (Standard Mean Ocean Water).

As análises de geoquímica de rocha total foram realizada no Laboratório da ALS Global em Vespasiano, Minas Gerais. As amostras foram pulverizadas em panela de vídia e entre cada pulverização, a panela foi descontaminada por meio de lavagem e pulverização de quartzo. Elementos maiores foram analisados por fluorescência de raio-X (XRF) pela fusão da rocha e perda ao fogo (LOI) foi determinada por fornalha e XRF. Os elementos Ba, Ce, Cr, Cs, Dy, Er, $\mathrm{Eu}, \mathrm{Ga}, \mathrm{Gd}, \mathrm{Hf}, \mathrm{Ho}, \mathrm{La}, \mathrm{Lu}, \mathrm{Nb}, \mathrm{Nd}$, Pr, Rb, Sm, Sn, Sr, Ta, Tb, Th, Tm, U, V, W, Y, Yb e Zr foram analisados por amostra fundida, digestão ácida(ácidos nítrico, hidroclórico e hidrofluórico) e finalização por espectrometria de massa com plasma acoplado induzido (ICPMS). Os elementos $\mathrm{Ag}, \mathrm{Cd}, \mathrm{Co}, \mathrm{Cu}, \mathrm{Li}, \mathrm{Mo}, \mathrm{Ni}, \mathrm{Pb}, \mathrm{Sc}$ e $\mathrm{Zn}$ foram analisados por digestão de quatro ácidos (acídos perclórico nítrico, hidroclórico e hidrofluórico) e finalização por espectroscopia de emissão atômica com plasma acoplado induzido (ICP-AES). Os elementos $\mathrm{As}, \mathrm{Bi}, \mathrm{Hg}, \mathrm{In}, \mathrm{Re}, \mathrm{Sb}, \mathrm{Sc}, \mathrm{Te}$ e $\mathrm{Tl}$ foram analisados por digestão por água régia e finalização por ICP-MS. Carbono e enxofre totais foram analisados por fornalha Leco. Amostras com teores maiores que $10.000 \mathrm{ppm}$ de $\mathrm{Zn}$ foram analizadas por digestão de quatro ácidos e finalização por espectroscopia de absorção atômica (AAS),e amostras com conteúdos de Zn acima de $30 \%$ foram analisadas por meio de titulação clássica de alta precisão.

Dados de química mineral e geoquímica de rocha total foram processados no programa ioGAS-64. 


\section{Capítulo 2}

\section{(ARTIGO A SER PUBLICADO): "Evolution of the Hydrothermal Systems in the Southern Extension of the Vazante Group: Implications to Silicate Zinc Mineralization"}

\subsection{INTRODUCTION}

Zinc is a metal of great importance for the industry and human health and it is the fourth most common metal in use, only behind iron, aluminum and copper, with an annual production of approximately 12 million tons (Tolcin, 2011). The main source of zinc is in the sulfide form (sphalerite $-\mathrm{ZnS}$ ) and the main zinc (and lead) deposits are hosted in sedimentary sequences, such as the Mississippi Valley type (MVT) and sedimentary exhalative (SEDEX) deposits. Together they contain around $48 \%$ and $52 \%$ of the global zinc and lead resources, respectively (Singer, 1995). The Irish type deposits are also expressive and exhibit characteristics considered transitional between the other two classes mentioned above (Wilkinson, 2014).

During the XIX century and beginning of the XX century, the main zinc source was supergene non-sulfide deposits, and smithsonite $\left(\mathrm{ZnCO}_{3}\right)$ was the main ore mineral. Later on, the sulfide deposits became the predominant source of zinc due to the development of differential flotation processes and of smelting and refining of sphalerite concentrates (Hitzman et al., 2003). However, the fact that the non-sulfide zinc deposits are of higher grade than the sulfide ones, and the recent metallurgic development of non-sulfide ore treatment and the lower environmental impact of this treatment have renewed the commercial interest on them as a potential great source of zinc in the XXI century (Brugger et al., 2003; Hitzman et al., 2003; Large, 2001). Despite the non-sulfide zinc deposits great potential, few studies were focused on the processes related to their formation and in particular of the hypogene deposits.

The Vazante Group is inserted on the Brasília Fold Belt (Fig. 2.1), and hosts the main zinc deposits of Brazil (Morro Agudo, Fagundes, Ambrósia and Vazante; Dardenne, 2000). The Vazante silicate zinc and the Morro Agudo zinc (lead) sulfide deposits are the only active mines in Brazil, operated by Votorantim Metais S.A. (Oliveira, 2013). Despite the recent commercial interest in silicate zinc deposits, the Vazante mine is on operation since the 1960's and it is considered to be the largest willemitic $\left(\mathrm{Zn}_{2} \mathrm{SiO}_{4}\right)$ deposit in the world, with estimated total resources of 40 to $60 \mathrm{Mt}$ at $20 \%$ of $\mathrm{Zn}$ (Baia, 2013). The Morro Agudo sulfide deposit contains measured reserves of $9.7 \mathrm{Mt}$ at $6.5 \%$ of $\mathrm{Zn}$ and $2.8 \%$ of $\mathrm{Pb}$ (Viviani et al., 2001). Various silicate zinc and sulfide zinc occurrences are reported in the Vazante Group, but their genetic relationships with the major deposits are poorly understood.

Many studies were performed focused mainly on the mineralogical, geochemical, and structural characteristics of the ore and related hydrothermal alterations in the Vazante Group (Amaral, 1968a; 1968b; Bettencourt et al., 2001; Bez, 1980; Carvalho et al., 1962; Dardenne, 1979; Dardenne and Schobbenhaus, 2001; Dresch, 1987; Hitzman, 1997; Hitzman et al., 1995; Misi et al., 1996; 2000; Monteiro et al., 1998a; Pinho, 1990, Pinho et al., 1989; Rigobello et al., 1988; Romagna and Costa, 1988; Slezak, 2012; Slezak et al., 2014) and fewer were focused on fluid inclusions and stable isotope geochemistry on the mineralized zones (Baia, 2013; Cunha, 1999; Cunha et al., 2000; 2001; Dardenne and Freitas-Silva, 1999; Misi et al., 1997; 1999; Monteiro, 1997; 2002; Monteiro et al., 1998b; 1999; 2003; 2006; 2007; Olivo et al., 2014). 
However, very little is known about the history of fluid-rock interaction in the Vazante Group prior- and post to the main mineralizingevent and their characteristics in barren zones.

The aim of this investigation is to document the various styles of alteration that occurred through the evolution of the Vazante Group (i.e., from diagenesis through late orogenic) in the barren and mineralized zones in the Lower Pamplona and Upper Morro do Pinheiro members of the Serra do Poço Verde Formation, Vazante Group, which are the main hosts for the zinc silicate deposits. The results are integrated with data from other deposits in the Vazante Group to constrain the major factors that controls economic concentrations of zinc in this geological setting.

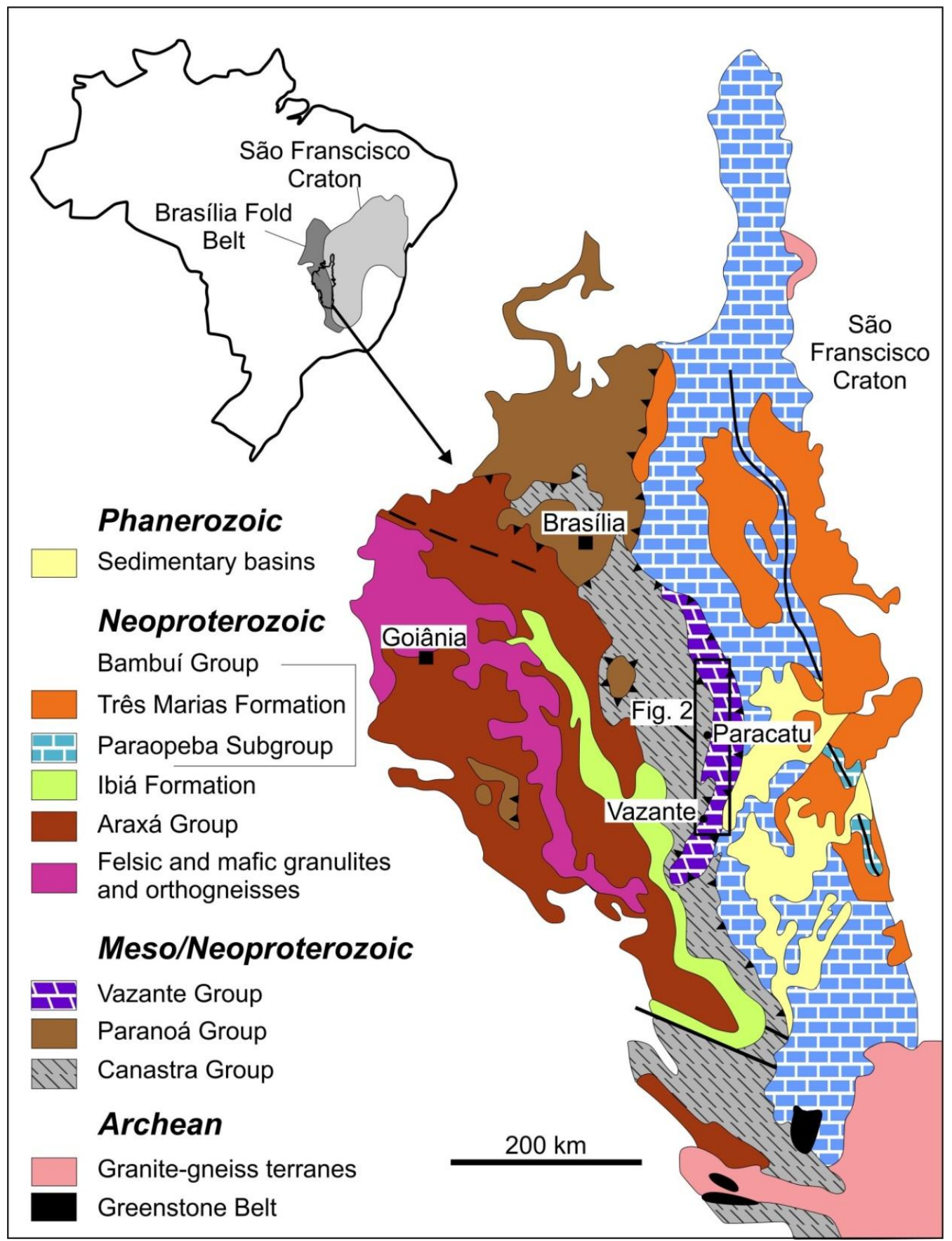

Fig. 2.1. Location map and geotectonic setting of the Vazante Group in the eastern part of the Brasília Fold Belt (Dardenne, 2000). 


\subsection{THE VAZANTE GROUP: GEOLOGICAL SETTING, STRATIGRAPHY AND DEFORMATION}

The Vazante Group is located in the Brasília Fold Belt, in the Tocantins Province, Central Brazil. According to Almeida and Hasui (1981), the Tocantins Province is a great dimension orogenesis that took place during the Neoproterozoic as a result of the convergence and collision of three important blocks: the Amazonas craton at northwest, the São FranciscoCongo craton at east and a supposed Paranapanema craton (Brito Neves et al., 1999), beneath the Phanerozoic sediments of the Paraná Basin, at southwest.

The Brasília Fold Belt (BFB) is at the east portion of the Tocantins Province and at the west border of the São Francisco craton (SFC). It extends for more than $1.000 \mathrm{~km}$ in an approximately N-S direction (Dardenne, 2000; Fuck, 1994; Marini et al., 1981). The BFB comprehends terranes and thrust slabs that converge to the east against the west portion of the São Francisco-Congo platform (Valeriano et al., 2008). According to Dardenne (2000), the main sedimentary units in the BFB are the thick metamorphosed sequences of the Serra da Mesa (Paleo/Mesoproterozoic), Paranoá and Canastra (Meso/Neoproterozoic), Araxá, Ibiá and Vazante (Meso/Neoproterozoic) and Bambuí (Neoporoterozoic) groups. The Vazante, Ibiá and Canastra groups form a complex system of nappes that were thrusted over the sedimentary rocks of the Bambuí Group at the east (Dardenne, 2000).

The Vazante Group is located in the northwest of Minas Gerais estate and its metasedimentary rocks cover an area of approximately $250 \mathrm{~km}$ along N-S-striking, with $25 \mathrm{~km}$ to $30 \mathrm{~km}$ average width. These rocks were affected by low greenschist facies metamorphism and by at least three deformation events (Monteiro, 1997; Monteiro et al., 2006; Slezak et al., 2014). The Vazante Group displays a bow shape with concavity towards the W and can be divided into two sectors with distinct stratigraphic, structural and metallogenetic features separated by the Rio Escuro Inflexion. On the northern sector are situated the sulfide-rich $\mathrm{Zn}$ and $\mathrm{Pb}$ deposits of Morro Agudo, Ambrósia and Fagundes, and on the southern sector is located the silicate Zn deposits (Vazante and North Extension) and occurrences (Mata II, Olho D'Água, Cercado, and Pamplona), mainly represented by the predominance of willemitic ore (Baia, 2013; Monteiro et al., 2006, 2007; Oliveira, 2013; Slezak et al., 2014).

The metasedimentary rocks of this group are composed of thick pelitic-dolomitic marine sequence, which can be divided into seven different formations, from the base to the top: Santo Antônio do Bonito, Rocinha, Lagamar, Serra do Garrote, Serra do Poço Verde, Morro do Calcário and Serra da Lapa formations (Dardenne et al., 1998; Dardenne, 2000; Misi et al., 2014). The main characteristics of these formations are summarized in Figure 2.2. Misi et al. (2014) have proposed that the upper part of the succession is late Mesoproterozoic in age and the basal is Neoproterozoic, based on Re-Os ages in organic-rich shales (Azmy et al., 2008; Geboy et al., 2013), and U-Pb data on detrital zircon in sandstones interspersed throughout the succession (Rodrigues et al., 2012; Fig. 2.2). It is suggested that the upper section (from Lagamar to Serra da Lapa formations) was thrusted over the lower counterpart (Santo Antônio do Bonito and Rocinha formations; see Misi et al., 2014, Fig. 7). The Serra do Poço Verde Formation, that is the focus of this investigation, is described in detail below.

The Serra do Poço Verde is in contact with the Serra do Garrote and Morro do Calcário formations, respectively in the bottom and top. It corresponds to a predominately dolomitic sequence and is divided in four members according to Rigobello et al., (1988) and Dardenne (2000), from the base to the top: Lower Morro do Pinheiro Member composed of laminated dolomite with cyanobacteria mats, intercalated with doloarenite and intraformational breccia levels associated with dolomite lenses and columnar stromatolites; Upper Morro do Pinheiro 
Member consists of laminated dolomite with stromatolitic mats and bird's eyes features intercalated with dolarenite, lamellar breccia and carbonaceous shale. The Lower Pamplona Member comprises siltstone and micritic dolomite, commonly pinkish, with cyanobacteria mats and lenses of fine sandstone and conglomerate; and Middle Pamplona Member consists of dolomite with stromatolitic mat laminations intercalated with dolarenite, lamellar breccia, columnar stromatolite dolomite and lenses of shale.

Sm-Nd analyses carried out on metasedimentary rocks from the Vazante Group indicate a uniform distribution of $T_{\mathrm{DM}}$ values between 2.100 and $1.700 \mathrm{Ma}$, which, according to Pimentel et al., 2001, reflect the overlapping of sources associated with the Paranoá $\left(T_{\mathrm{DM}}=2.300\right.$ to $2.000 \mathrm{Ma})$ and Bambuí groups $\left(T_{\mathrm{DM}}=1.900\right.$ to $\left.1.400 \mathrm{Ma}\right)$. This is compatible with an intermediate stratigraphic position of the Vazante Group, which could correspond to the top of the Paranoá passive margin sequence (Pimentel et al., 2001), or could alternatively represent the sedimentation in a rapidly subsiding zone forming a depression in the Brasília Fold Belt initial thrust fronts (Dardenne, 2000; Monteiro et al., 2006). A Rb-Sr whole rock isochron for shales from the Vazante Group yielded an age of 600 50 Ma (Amaral and Kawashita, 1967), which could represent the last closing of the isotopic systems, during the Brasiliano metamorphic event (Monteiro et al., 2006). 


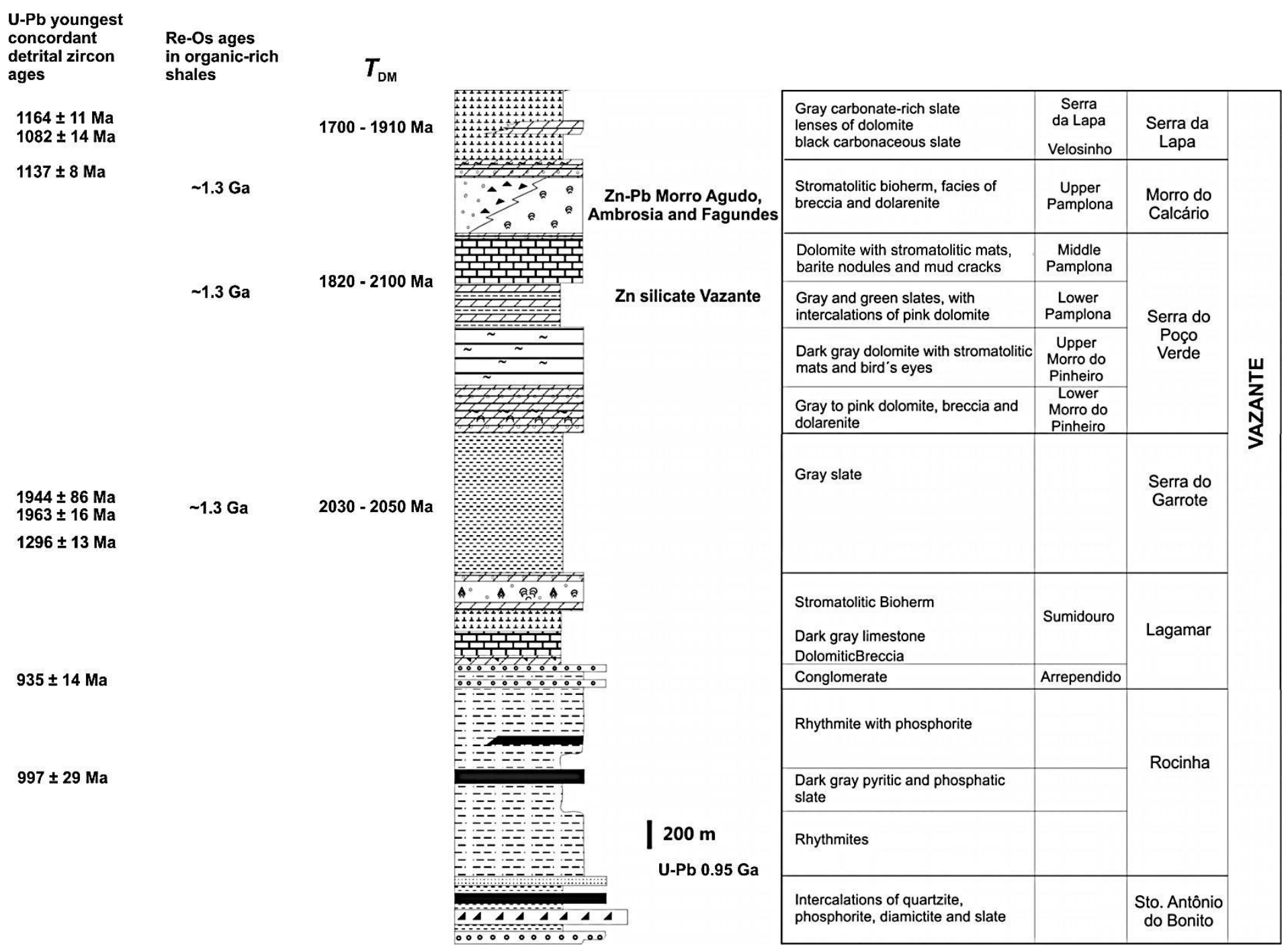

Fig. 2.2. Schematic diagram of the lithostratigraphic framework of the Vazante Group (after Dardenne, 2001 and Misi et al., 2014). U-Pb detrital zircon ages from Rodrigues et al., (2012); ReOs ages from Geboy et al., (2013) and Azmy et al., (2008); $T_{\mathrm{DM}}$ ages from Pimentel et al., (2001). 


\subsection{VAZANTE GROUP METAL DEPOSITS}

The major characteristics of the hypogene silicate zinc (southern part) and sulfide leadzinc (northern part) deposits and occurrences of the Vazante Group are summarized in Table 2.1, and their locations are shown in Figure 2.3. This study was focused mainly on the southern part of the group where there are occurrences of the hypogene silicate mineralization, and the major features associated with this type of deposit in the region are summarized below.

The Vazante Deposit comprises zinc ore constituted mainly by hypogene willemite and minor sphalerite and franklinite, and supergene hemimorphite (Monteiro et al., 2006; Slezak et al., 2014). The orebodies occur within major tectonic-hydrothermal breccias controlled by a NE-trending fault zone. The faults and the breccias are interpreted to be formed during the $\mathrm{D}_{2}$ deformation event, which is related to the Neoproterozoic Brasiliano Orogeny. The faults were reactivated during their evolution and are cut by later transcurrent faulting (Slezak et al., 2014).

The bulk of the Vazante non-sulfide $\mathrm{Zn}$ ore is concentrated close to the contact of the Upper Morro do Pinheiro Member (footwall sequence) and the overlying Lower Pamplona Member, within the Vazante Fault Zone (Monteiro et al., 2006). Small diabasic bodies occur tectonically imbricated with brecciated metadolomites and willemitic ore bodies along the fault zone (Monteiro, 1997). The Vazante Fault Zone is interpreted to have undergone various episodes of reactivation, including inverse and transcurrent movements during a compressive phase that, later on, was reactivated as a normal fault at the final stages of the Brasiliano Orogeny (Dardenne, 2000; Monteiro et al., 1999; Pinho et al., 1990).

The hydrothermal alteration is largely fracture controlled, producing a complex zone of net veined and hydraulic breccias. The metadolomites are also bleached and metasomatically altered along the contact with the gabbroic bodies (Monteiro et al., 2006). The hydrothermalized metadolomite displays colour variability due to the precipitation of minerals such as $\mathrm{Fe}$ dolomite, siderite, and saddle dolomite. Silicification is pervasive in strongly brecciated zones cut by hydrothermal veins. These veins display a crustiform texture related to progressive infilling of open fractures by successive layers of texturally and/or mineralogically distinct precipitates, which include Fe-dolomite, siderite, jasper, hematite and chlorite (Monteiro, 2002; Monteiro et al., 2006).

The main hypogenic willemitic mineralization in the Vazante Deposit displays podiform morphology and comprises willemite, hematite, siderite and dolomite, with subordinate franklinite, zincite, magnetite, chlorite, barite, smithsonite and apatite. It is tectonically imbricated with restrict sulfide bodies with sphalerite and galena hosted by intensely hydrothermalized brecciated dolomite. The mineralizing stage was considered late to the metamorphic peak, synchronous to the development of the fault zone (Monteiro, 2002). 


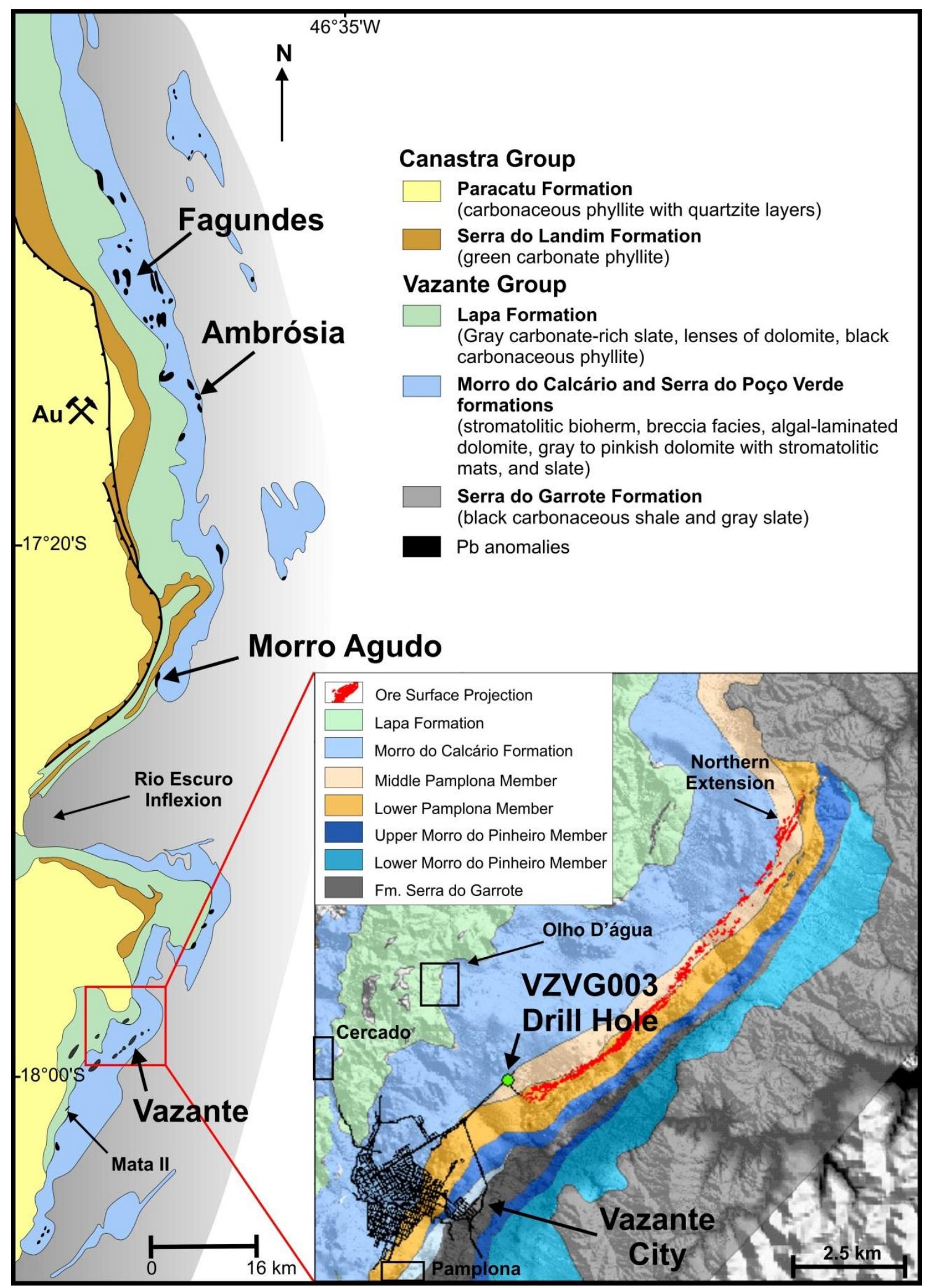

Fig. 2.3. Geological map of the Vazante Group and the location of the main base metals deposits and occurrences of the drill core studied. Modified from Monteiro et al., 2006 and compiled from Votorantim Metais data, 2015. 
Table 2.1. Major characteristics of hypogene sulfide lead-zinc and silicate zinc deposits and occurrences in the Vazante Group.

\begin{tabular}{|c|c|c|c|c|c|c|c|}
\hline \multicolumn{8}{|c|}{ Sulfide-rich deposits in the Vazante Group } \\
\hline Deposit & Host Rocks & Ore Control & $\begin{array}{l}\text { Hydrothermal } \\
\text { Alteration }\end{array}$ & $\begin{array}{l}\text { Styles of } \\
\text { Mineralization }\end{array}$ & Ore Types & Mineralogy $S$ & Sulfur Source \\
\hline $\begin{array}{l}\text { Morro } \\
\text { Agudo }^{(a)}\end{array}$ & $\begin{array}{l}\text { Breccia, } \\
\text { dolarenites } \\
\text { and dolorudites } \\
\text { (Upper Pamplona } \\
\text { Member) }\end{array}$ & $\begin{array}{l}\text { Fault zone } \\
\text { (N10W/75SW); } \\
\text { stratigraphic } \\
\text { control }^{(3,4)}\end{array}$ & $\begin{array}{l}\text { Silicification } \\
\text { posterior to the } \\
\text { main } \\
\text { mineralization }\end{array}$ & $\begin{array}{l}\text { Syn- } \\
\text { diagenetic/ } \\
\text { epigenetic }\end{array}$ & $\begin{array}{l}\text { Stratiform ore, } \\
\text { breccia ore and }_{\text {veins }^{(3)}}\end{array}$ & $\begin{array}{ll}\text { gn, sph, py, } & \mathrm{T} \\
\text { cal, dol(3), } & \text { t } \\
\text { brt, qtz } & 0\end{array}$ & $\begin{array}{l}\text { Two sulfur sources: from } \\
\text { thermochemical reduction } \\
\text { of evaporitic sulfate and } \\
\text { hydrothermal supply } \\
\text { (3,4,5) }\end{array}$ \\
\hline Fagundes ${ }^{(\mathbf{a})}$ & $\begin{array}{l}\text { Dolarenite, } \\
\text { dolorudite, } \\
\text { dolarenitic breccia } \\
\text { (Upper Pamplona } \\
\text { Member) }\end{array}$ & $\begin{array}{l}\text { Stratigraphic } \\
\text { control; } \\
\text { brittle-ductile } \\
\text { fault zones }\end{array}$ & $\begin{array}{l}\text { Strong } \\
\text { silicification } \\
\text { and baroque } \\
\text { dolomite } \\
\text { formation }^{(1)}\end{array}$ & $\begin{array}{l}\text { Tardi- } \\
\text { diagenetic/ } \\
\text { epigenetic }\end{array}$ & $\begin{array}{l}\text { Stratabound ore } \\
\text { (open space filling, } \\
\text { largely replacive), } \\
\text { brecciated ore }\end{array}$ & $\begin{array}{l}\text { py, sp, gn, } \\
\text { mrc, dol, } \\
\text { qtz, phyll, } \\
\text { chalcedony }{ }^{(1)} \text { r } \\
\\
\text { c } \\
\text { h }\end{array}$ & $\begin{array}{l}\text { Two sulfur sources: } \\
\text { thermochemical } \\
\text { reduction of sulfate } \\
\text { under closed system } \\
\text { conditions and } \\
\text { hydrothermal supply }\end{array}$ \\
\hline Ambrósia ${ }^{(\mathbf{a})}$ & $\begin{array}{l}\text { Brecciated } \\
\text { dolomite } \\
\text { (Upper Pamplona } \\
\text { Member) }\end{array}$ & $\begin{array}{l}\text { High angle fault } \\
\text { zone } \\
\text { (N30W/80SW) }\end{array}$ & $\begin{array}{l}\text { Replacement } \\
\text { by baroque } \\
\text { dolomite } \\
\text { and ankerite, } \\
\text { silicification }\end{array}$ & Epigenetic & Lode ore & $\begin{array}{l}\text { py, sp, gn, } \\
\text { dol, qtz, } \\
\text { marc, } \\
\text { phyll, ap }\end{array}$ & Hydrothermal source ${ }^{(2)}$ \\
\hline Mata II (b) & $\begin{array}{l}\text { Brecciated } \\
\text { dolomite } \\
\text { (Morro do } \\
\text { Calcário/Lapa } \\
\text { formations) }\end{array}$ & $\begin{array}{l}\text { Fault zone } \\
\text { (N25E/40NW); } \\
\text { stratigraphic } \\
\text { control }^{(8)}\end{array}$ & $\begin{array}{l}\text { Replacement } \\
\text { by jasper and } \\
\text { hematite }\end{array}$ & Epigenetic & $\begin{array}{l}\text { Replacive and } \\
\text { brecciated ore }\end{array}$ & $\begin{array}{l}\text { qtz, hem, } \\
\text { Fe-oxi/ } \\
\text { hydroxide, } \\
\text { Chl, K-F, } \\
\text { gn, cv, cpr }{ }^{(8)}\end{array}$ & * \\
\hline $\begin{array}{l}\text { Olho } \\
\text { D'água }^{(b)}\end{array}$ & $\begin{array}{l}\text { Brecciated } \\
\text { dolomite } \\
\text { (Morro do } \\
\text { Calcário/Lapa } \\
\text { formations) }\end{array}$ & $\begin{array}{l}\text { Fault zone } \\
\text { (N45E/30NW); } \\
\text { stratigraphic } \\
\text { control }^{(8)}\end{array}$ & $\begin{array}{l}\text { Replacement } \\
\text { by hematite, } \\
\text { dolomite and } \\
\text { jasper }\end{array}$ & Epigenetic & $\begin{array}{l}\text { Replacive and } \\
\text { brecciated ore }\end{array}$ & $\begin{array}{l}\text { Zn-dol, hem, } \\
\text { jasper, sid, } \\
\text { will, fk, } \\
\text { Zn-chl, ap }{ }^{(8)}\end{array}$ & * \\
\hline Cercado $^{(\mathbf{b})}$ & $\begin{array}{l}\text { Brecciated } \\
\text { dolomite } \\
\text { (Serra do Poço } \\
\text { Verde and Morro } \\
\text { do Calcário/Lapa } \\
\text { formations) }\end{array}$ & $\begin{array}{l}\text { High angle } \\
\text { normal } \\
\text { fault zone } \\
(\mathrm{N} 35 \mathrm{E} / 75 \mathrm{NW})^{(\mathbf{8 )}}\end{array}$ & $\begin{array}{l}\text { Replacement } \\
\text { by dolomite } \\
\text { and hematite }\end{array}$ & Epigenetic & $\begin{array}{l}\text { Replacive and } \\
\text { brecciated ore, } \\
\text { veins }\end{array}$ & $\begin{array}{l}\text { Zn-dol, hem, } \\
\text { qtz, Zn-chl, } \\
\text { will, fk, } \\
\text { Fe-oxi/ } \\
\text { hydroxide, } \\
\text { ser, zc, sp, gn }{ }^{(8)}\end{array}$ & $\begin{array}{l}* \\
(8)\end{array}$ \\
\hline Pamplona $a^{(\mathbf{b})}$ & $\begin{array}{l}\text { Brecciated } \\
\text { dolomite } \\
\text { (Serra do Poço } \\
\text { Verde/Morro } \\
\text { do Calcário) }\end{array}$ & $\begin{array}{l}\text { High angle } \\
\text { normal } \\
\text { fault zone } \\
(\mathrm{N} 70 \mathrm{E} / 60 \mathrm{NW})^{(8)}\end{array}$ & $\begin{array}{l}\text { Replacement } \\
\text { by Fe-dolomite } \\
\text { and hematite }\end{array}$ & Epigenetic & $\begin{array}{l}\text { Replacive and } \\
\text { brecciated ore, } \\
\text { veins }\end{array}$ & $\begin{array}{l}\text { Zn-dol, hem, } \\
\text { will, jasper, } \\
\text { Fe-oxi// } \\
\text { hydroxide }^{(8)}\end{array}$ & $*$ \\
\hline $\begin{array}{l}\text { Vazante }^{\odot} \\
\text { and } \\
\text { Nothern } \\
\text { Extension }^{\odot}\end{array}$ & $\begin{array}{l}\text { Brecciated } \\
\text { pink dolomite } \\
\text { and slates } \\
\text { (Serra do Poço } \\
\text { Verde/Morro } \\
\text { do Calcário) }\end{array}$ & $\begin{array}{l}\text { Shear zone } \\
\left(\mathrm{N} 50 \mathrm{E} / 60 \mathrm{NW}{ }^{(1)}\right. \\
\left(\mathrm{N} 30 \mathrm{E} / 30 \mathrm{NW}{ }^{(9)}\right. \\
\text { and } \mathrm{N} 30 \mathrm{E} / 55 \mathrm{SE})^{(9)} \\
\text { stratigraphic } \\
\text { control }\end{array}$ & $\begin{array}{l}\text { Silicification, } \\
\text { dolomitization, } \\
\text { siderite, ankerite, } \\
\text { hematite, cholrite } \\
\text { formation }^{(6,7)}\end{array}$ & Epigenetic & $\begin{array}{l}\text { Willemitic ore } \\
\text { (pods, lode, } \\
\text { breccia) and } \\
\text { sulfide ore } \\
\text { (veins, pods) }\end{array}$ & $\begin{array}{l}\text { Will, hem, } \\
\text { dol, sid, } \\
\text { ank, sp, gn, } \\
\text { fk, zc, qtz, } \\
\text { chl, brt, ap, } \\
\mathrm{Ag}^{0}, \text { cv }^{(6,7,9)}\end{array}$ & $\begin{array}{l}\text { Thermochemical reduction } \\
\text { of seawater sulfate or } \\
\text { hydrothermal source }\end{array}$ \\
\hline
\end{tabular}

Abbreviations: $\mathrm{py}=$ pyrite; $\mathrm{sp}=$ phalerite; $\mathrm{gn}=$ galena; dol = dolomite; qtz = quartz; $\mathrm{mrc}=$ marcasite; phyll = phyllosilicate; cal = calcite; brt = barite; ap = apatite (a) = sulfide $\mathrm{Zn}-\mathrm{Pb}$; (b) silicate $\mathrm{Zn}$ occurrences; (c) = silicate $\mathrm{Zn}$ mines; (1) Monteiro et al., 2006; (2) Monteiro and Bettencourt, 2001; (3) Cunha et al., 2000;(4) Misi et al., 1999;(5) Misi et al., 2000; (6) Monteiro, 1997; (7) Monteiro et al., 1999; (8) Baia, 2013; (9) Slezak et al., 2013.

Slezak (2012) and Slezak et al., (2014) studied the Northern Extension Vazante Deposit where the mineralization is constrained to a fault zone that cuts through the Serra do Poço Verde and Morro do Pinheiro formations. There is a sequence of hydrothermal breccia types from distal poorly brecciated and hydrothermalized dolomite with Fe-carbonates passing through an increasing brecciation more proximal to the main ore zone with Fe-carbonate alteration and substitution by hematite. The ore breccia, located at the core of the tectonic-hydrothermal breccia structure, comprises three generations of willemite, hematite and lesser amounts of franklinite, ferroan and zincian dolomite and quartz. Late sphalerite, galena, native silver, chalcocite, stromeyerite, covellite veinlets cut the willemitic ore.

Baia (2013) studied other exploratory targets near the Vazante mine (Mata II, Olho D'Água, Cercado and Pamplona). On the Mata II and Olho D'Água occurrences, the hydrothermal brecciation is associated to fault zones within the contact of the Morro do Calcário and Serra da Lapa formations. The hydrothermal cement of the mineralized hydrothermal breccia on the Mata II target is composed of quartz, hematite, Fe-oxi/hydroxides and subordinate chlorite, K-feldspar, galena, covellite and cuprite, with no $\mathrm{Zn}$-bearing mineral. The 
Olho D'Água target is composed of coarse white $\mathrm{Zn}$-dolomite, jasper, hematite, siderite and locally willemite, and minor franklinite, Zn-bearing chlorite and subordinate apatite. In the Cercado occurrence the mineralization is controlled by a normal fault at the contact of the Morro do Calcário and Serra do Poço Verde formations at the base, and Serra da Lapa Formation at the top (Baia, 2013). The mineralized breccia comprises Zn-dolomite, hematite, Zn-chlorite, quartz, willemite, minor franklinite, Fe-oxi/hydroxides, sericite, zincite, sphalerite, and galena veinlets. The Pamplona target is controlled by a fault zone where the Morro do Calcário and Serra do Poço Verde formations are juxtaposed (Baia, 2013). The hydrothermal breccia is composed by pink ferroan dolomite and hematite veinlets. The $\mathrm{Zn}$ mineralization is mainly willemitic associated to ferroan dolomite and jasper with moderately developed mylonitic foliation.

The age of the base metal mineralization is poorly constrained. Lead isotopic $\left({ }^{207} \mathrm{~Pb} /{ }^{206} \mathrm{~Pb}\right)$ analyses of galena from Vazante and Morro Agudo deposits yielded model ages based on Stacey and Kramers (1975) ranging between 929 and 600 Ma (Amaral, 1968a; Cassedane and Lasserre, 1969; Cunha et al., 2001, 2003; Freitas-Silva and Dardenne, 1997; Iyer et al., 1992, 1993; Misi et al., 1997), which have been considered as the time of mineralization (Iyer et al., 1992, 1993; Misi et al., 1997) or as the time of lead separation from the source. However, according to Cunha et al. (2003) and Babinski et al. (2005), the Pb-isotopic ratios of galena from the Vazante-Paracatu region plot above of the evolution curve of Stacey and Kramers (1975), indicating that the obtained ages lack geologic significance (Monteiro, et al., 2006). Additional analyses on galena from the Morro Agudo Deposit resulted in $\mathrm{Pb}-\mathrm{Pb}$ plumbotectonic model ages of 1.200 to 1.000 Ma for early fine-grained galena, which have been interpreted as the time of lead separation or as the time of the syn-diagenetic mineralization, and of $600 \mathrm{Ma}$ for epigenetic coarse-grained galena, considered as the time of epigenetic mobilization processes (Dardenne and Schobbenhaus, 2001; Freitas-Silva and Dardenne, 1997).

\subsection{PREVIOUS FLUID INCLUSION SUDIES IN THE VAZANTE GROUP}

Fluid inclusion studies in the Vazante Group were mainly focused on the mineral phases associated with ore bodies in Morro Agudo (Cunha, 1999; Cunha et al., 2000), Fagundes, Ambrósia, and Vazante deposits (Dardenne and Freitas-Silva, 1999; Monteiro, 2002). Compilation of fluid inclusion data is shown on Table 2.2 and discussed below.

Fluid inclusions from the mineralizing stage in the Morro Agudo Deposit comprise $\mathrm{NaCl}-\mathrm{H}_{2} \mathrm{O}$ fluids with low to moderate salinities (average values between 4.8 and 12.85 wt. \% $\mathrm{NaCl}$ equiv.) and with higher temperatures (up to $300^{\circ} \mathrm{C}$ ) closer to the fault zone and lower temperatures in the distal zones (down to $80^{\circ} \mathrm{C}$; Cunha et al., 2000). The salinity is also higher closer to the fault zone (up to $32 \mathrm{wt}$. $\% \mathrm{NaCl}$ equiv.) with lower values away from the fault zone (down 0 wt. \% NaCl equiv.). This is consistent with isotopic data from cogenetic pairs of sphalerite and galena from Misi et al. (1996). Cunha et al., (2000) suggested that higher temperature and salinity fluids next to the site of higher discharge have mixed with basinal fluids of low to moderate salinities, what resulted in a decrease of the temperature and salinities away from the fault zone. 
Table 2.2. Fluid inclusion characteristics from the zinc (and lead) deposits of the Vazante Group.

\begin{tabular}{|c|c|c|c|c|}
\hline Deposit & Fluid Inclusion & $\begin{array}{l}\text { Eutectic } \\
\text { Temp. }\left({ }^{\circ} \mathrm{C}\right)\end{array}$ & $\begin{array}{l}\text { Homog. } \\
\text { Temp. }\left({ }^{\circ} \mathrm{C}\right)\end{array}$ & $\begin{array}{l}\text { Sal. (wt \% of } \\
\text { NaCl equiv.) }\end{array}$ \\
\hline \multirow[t]{2}{*}{ Morro Agudo $^{(\mathbf{1})}$} & $\begin{array}{l}\text { Primary, hosted in sphalerite proximal } \\
\text { to the main fault zone (interpreted } \\
\text { feeder zone) }\end{array}$ & $\begin{array}{l}-21.2^{\circ} \\
\text { (temperatures } \\
\text { below were } \\
\text { discarted) }\end{array}$ & $\begin{array}{l}80 \text { to } 300 \\
(\text { mode }=130 \\
\text { to } 170)\end{array}$ & $\begin{array}{l}\text { Mode }=11.7 \text { to } \\
12.85 \text { (up to } \\
32 \text { wt. } \% \text { ) }\end{array}$ \\
\hline & $\begin{array}{l}\text { Primary, hosted in sphalerite distal } \\
\text { from the main fault zone (interpreted } \\
\text { feeder zone) }\end{array}$ & Idem & $\begin{array}{l}80 \text { to } 210 \\
(\text { mode }=130 \\
\text { to } 170)\end{array}$ & $\begin{array}{l}\text { Mode }=4.8 \text { to } \\
7.8 \text { (down to } \\
0 \text { wt. } \%)\end{array}$ \\
\hline \multirow[t]{6}{*}{ Fagundes $^{(2)}$} & $\begin{array}{l}\text { Primary in pre-mineralization } \\
\text { quartz }\end{array}$ & -46.0 to -35.5 & 143.5 to 190.6 & 5.9 to 14.2 \\
\hline & $\begin{array}{l}\text { Primary in syn-mineralization } \\
\text { sphalerite and quartz }\end{array}$ & -51.7 to -32.8 & 138.9 to 260.2 & 5.8 to 11.8 \\
\hline & $\begin{array}{l}\text { Secondary in syn-mineralization } \\
\text { sphalerite and quartz }\end{array}$ & -53.4 to -33.8 & 91.1 to 186.6 & 4.1 to 13.7 \\
\hline & $\begin{array}{l}\text { Primary in mobilized sphalerite } \\
\text { and dolomite }\end{array}$ & -57.2 to -44.2 & 127.8 to 264.7 & 5.2 to 15.6 \\
\hline & $\begin{array}{l}\text { Secondary in mobilized } \\
\text { sphalerite, dolomite and quartz }\end{array}$ & -55.4 to -40.3 & 97.1 to 197.2 & 0.0 to 15.7 \\
\hline & $\begin{array}{l}\text { Secondary in late } \\
\text { mineralization sphalerite }\end{array}$ & -52.3 to 40.3 & & 6.4 to 12.7 \\
\hline \multirow[t]{5}{*}{ Ambrósia $^{(2)}$} & $\begin{array}{l}\text { Primary in syn-mineralization } \\
\text { sphalerite }\end{array}$ & -55.6 to -48.5 & 167.8 to 281.6 & 14.6 to 20.2 \\
\hline & $\begin{array}{l}\text { Secondary in syn- } \\
\text { mineralization sphalerite }\end{array}$ & -55.6 to 49.8 & 106.4 to 140.9 & 6.4 to $>23.1$ \\
\hline & Primary in mobilized quartz & -53.6 to -46.5 & 241.6 to 243.5 & 16.7 to 21.9 \\
\hline & Secondary in mobilized quartz & -55.4 to -40.6 & 114.7 to 137.9 & 0.5 to 12.2 \\
\hline & $\begin{array}{l}\text { Primary in late mineralization } \\
\text { sphalerite }\end{array}$ & -65.1 to -61.0 & 105.2 to 136.2 & 16.7 to $\sim 23.1$ \\
\hline \multirow[t]{5}{*}{ Vazante } & Willemite $^{(3)}$ & & 65.0 to 180.0 & 3.0 to 15 \\
\hline & $\begin{array}{l}\text { Primary in syn-mineralization } \\
\text { sphalerite }^{(2)}\end{array}$ & -53.8 to -41.3 & 170.0 to 232.1 & 15.3 to $>23.0$ \\
\hline & $\begin{array}{l}\text { Secondary in syn- } \\
\text { mineralization sphalerite }\end{array}$ & -53.5 to -52.3 & 100.5 to 165.0 & 10.5 to 15.0 \\
\hline & $\begin{array}{l}\text { Primary and pseudo-secondary } \\
\text { in post-mineralization quartz }\end{array}$ & -52.2 to -37.0 & 135.0 to 160.0 & 6.7 to 18.0 \\
\hline & $\begin{array}{l}\text { Secondary in post- } \\
\text { mineralization quartz }\end{array}$ & -54.5 to -36.6 & 75.0 to 140.0 & 6.5 to 20.4 \\
\hline
\end{tabular}

Abbreviations: Temp. $=$ Temperature; Homg. $=$ Homogenization, Sal. $=$ Salinity, $\operatorname{Tm}($ ice $)=$ temperature of final ice melting.

(1) Cunha et al., 2000; (2) Monteiro, 2002: (3) Dardenne and Freitas-Silva, 1999.

Primary mineralization (epigenetic) in the Ambrósia Deposit has more saline (14.6 to 20.2 wt. $\% \mathrm{NaCl}$ equiv.) fluids than the late-diagenetic fluids of the Fagundes Deposit (5.8 to 11.8 wt. $\% \mathrm{NaCl}$ equiv.), and late (epigenetic) mineralization (5.2 to 15.6 wt. $\% \mathrm{NaCl}$ equiv.) in Fagundes Deposit (Monteiro, 2002). 
Monteiro (2002) proposed that $\mathrm{H}_{2} \mathrm{O}-\mathrm{NaCl}-\mathrm{CaCl}_{2}$ is the predominant fluid composition for the Ambrósia, Fagundes and Vazante deposits and may represent the main composition of basinal brines (Goldstein and Reynolds, 1994; Wilkinson, 2001). According to Monteiro (2002) fluids show the presence of $\mathrm{MgCl}_{2}$ and possibly $\mathrm{FeCl}_{2}$ (eutectic temperatures up to $-32.8^{\circ} \mathrm{C}$ ) and the mineralizing stage is characterized by $\mathrm{Ca}^{2+}$ richer fluids, alike the described for other carbonate sequence hosted deposits, in which the main mineralizing stage is marked by an increment in the $\mathrm{CaCl}_{2} / \mathrm{NaCl}$ ratios, possibly due to dissolution and re-precipitation of carbonate.

Fluid inclusion studies conducted on the Vazante Deposit by Dardenne and FreitasSilva (1999) revealed that the fluids related to willemite show salinities of 3 to 15 wt. $\% \mathrm{NaCl}$ equiv. and homogenization temperatures of $65^{\circ}$ to $180^{\circ} \mathrm{C}$. Studies of sphalerite fluid inclusion from this deposit, conducted by Monteiro (2002), indicate salinities around 15 to $23 \mathrm{wt} . \% \mathrm{NaCl}$ equiv. and homogenization temperatures ranging from $201^{\circ}$ to $232^{\circ} \mathrm{C}$. However, the overall fluids from the zinc (and lead) deposits in the Vazante Group show salinities values predominantly between 4 and 10 wt. $\% \mathrm{NaCl}$ equiv. and homogenization temperatures between $130^{\circ} \mathrm{C}$ to $190^{\circ} \mathrm{C}$ (Monteiro, 2002). Upon these data, this author interpreted that fluid mixing could have been important during deposition, and three different types can be distinguished regionally: (i) fluid of anomalous high temperatures $\left(>200^{\circ} \mathrm{C}\right)$ with higher eutectic temperatures, low to moderate salinities, considered metalliferous, with important role on the initial mineralizing stages; (ii) fluid of moderate temperatures $\left(140^{\circ} \mathrm{C}\right.$ to $\left.190^{\circ} \mathrm{C}\right)$, close to the $\mathrm{H}_{2} \mathrm{O}-\mathrm{NaCl}-\mathrm{CaCl}_{2}$ system that could have played an important role on the late mineralizing (epigenetic) episodes; and (iii) low to moderate temperatures $\left(90^{\circ} \mathrm{C}\right.$ to $160^{\circ} \mathrm{C}$ ), with low salinity, possibly with meteoric contribution.

\subsection{PREVIOUS CARBON AND OXYGEN ISOTOPIC STUDIES IN THE VAZANTE GROUP}

Not many oxygen stable isotopic data are available for the zinc mineralized occurrences at the Vazante Group (Baia, 2013; Monteiro, 1997; 2002; Monteiro et al., 1999; 2007; Percy, 2014; Rubo and Monteiro, 2010) and host rocks (Oliveira, 2013) and these data are summarized on Table 2.3 and briefly discussed below.

According to Rubo and Monteiro (2010), significant variation of $\delta^{18} \mathrm{O}$ values $(+19.7$ to $+27.0 \%$ SMOW) from the matrix of the non-hydrothermalized rocks from the Morro Agudo Deposit suggests, even though there is no observed textural change, that these rocks might have interacted with hydrothermal fluids. The lowest value for the least altered dolostones from the Fagundes Deposit $\left(\delta^{13} \mathrm{C}=-1.3 \%\right.$ PDB $)$ might reflect variable degree of organic matter oxidation (Burns et al., 1988; Monteiro et al., 2007).

According to Monteiro et al., (2007), the decrease of $\delta^{18} \mathrm{O}$ values (down to $+15.1 \%$ ) in hydrothermalized dolostones from the Ambrósia and Vazante deposits reflect the interaction with hydrothermal fluids at relatively high temperatures. However narrow range of $\delta^{18} \mathrm{O}$ values $(+12.4$ to $+19.9 \%)$ in hydrothermalized dolostones at the Fagundes Deposit could result from homogenization of the oxygen isotope compositions due to high fluid/rock ratios.

Monteiro et al.- (2007) suggest that non-sulfide zinc mineralization gangue carbonate isotope data indicate fluid mixing process of regional metalliferous fluid with isotopically light oxidizing meteoric water, especially in the late mineralization stages and post-mineralization mobilization. 
Table 2.3. Compilation of the stable isotope data from the zinc (and lead) deposits from the Vazante Group.

\begin{tabular}{|c|c|c|c|c|c|c|c|}
\hline \multirow[b]{2}{*}{ Sample } & \multirow[b]{2}{*}{ Deposit } & \multirow[b]{2}{*}{$\delta^{13} \mathrm{C}(\%)$} & \multirow[b]{2}{*}{$\delta^{18} \mathrm{O}(\%)$} & \multicolumn{2}{|c|}{$150^{\circ} \mathrm{C}$} & \multicolumn{2}{|l|}{$200^{\circ} \mathrm{C}$} \\
\hline & & & & $\begin{array}{l}\delta^{13} \mathrm{C}(\%) \\
\text { Fluid }\end{array}$ & $\begin{array}{l}\delta^{18} \mathrm{O}(\%) \\
\text { Fluid } \\
\end{array}$ & $\begin{array}{l}\delta^{13} \mathrm{C}(\%) \\
\text { Fluid } \\
\end{array}$ & $\begin{array}{l}\delta^{18} \mathrm{O}(\%) \\
\text { Fluid } \\
\end{array}$ \\
\hline \multirow{5}{*}{$\begin{array}{l}\text { Least Altered } \\
\text { Dolostones }\end{array}$} & Vazante $^{(4)}$ & 0.0 to +4.0 & +24.0 to +30.0 & * & * & * & * \\
\hline & Morro Agudo $^{(2)}$ & -0.6 to +0.5 & +19.7 to +27.0 & $*$ & $*$ & * & $*$ \\
\hline & Fagundes $^{(1)}$ & -1.3 & +25.8 & * & $*$ & $*$ & $*$ \\
\hline & Ambrósia ${ }^{(1)}$ & +0.3 to +0.7 & +28.6 to +29.7 & $*$ & $*$ & * & * \\
\hline & $\begin{array}{l}\text { Mata II, Olho D'água, } \\
\text { Cercado and Pamplona }\end{array}$ & -0.5 to +2.7 & +22.1 to +28.1 & $*$ & $*$ & $*$ & $*$ \\
\hline \multirow{8}{*}{$\begin{array}{l}\text { Gangue } \\
\text { Carbonate }\end{array}$} & Vazante Willemitic Ore $^{(1)}$ & +0.3 to +0.9 & +17.4 to +20.4 & -1.6 to -1.0 & +3.0 to +6.0 & +0.3 to +0.9 & +6.5 to +9.5 \\
\hline & Vazante Sulfide Ore $^{(\mathbf{l})}$ & -6.0 to +1.7 & +21.6 to +31.9 & -7.9 to -0.2 & +7.2 to +17.5 & -6.0 to +1.7 & +10.7 to +21.0 \\
\hline & $\begin{array}{l}\text { Vazante Northern } \\
\text { Extension (Willemitic) }{ }^{(3)}\end{array}$ & +0.1 to +1.3 & +15.8 to +20.8 & -1.8 to -0.6 & +1.4 to +6.4 & +0.1 to +1.3 & +4.9 to +9.9 \\
\hline & $\begin{array}{l}\text { Vazante Northern } \\
\text { Extension (Sulfide) }{ }^{(3)}\end{array}$ & -2.3 to +0.1 & +21.9 to +22.0 & -4.2 to -1.8 & +7.5 to +7.6 & -2.3 to +0.1 & +11.0 to +11.1 \\
\hline & Morro Agudo ${ }^{(2)}$ & -1.2 to +1.0 & +15.5 to +22.8 & -3.1 to -0.9 & +1.1 to +8.4 & -1.2 to +1.0 & +4.6 to +11.9 \\
\hline & Fagundes ${ }^{(1)}$ & -2.3 to +0.2 & +12.4 to +19.9 & -4.2 to -1.7 & -2.0 to +5.5 & -2.3 to +0.2 & +1.5 to +9.0 \\
\hline & Ambrósia ${ }^{(1)}$ & -2.3 to +0.3 & +15.1 to +20.3 & -4.2 to -1.6 & +0.7 to +5.9 & -2.3 to +0.3 & +4.2 to +9.4 \\
\hline & $\begin{array}{l}\text { Mata II, Olho D'água, } \\
\text { Cercado and Pamplona }{ }^{(5)}\end{array}$ & -4.1 to +0.8 & +15.5 to 23.8 & -6.0 to -1.1 & +1.1 to +9.4 & -4.1 to +0.8 & +4.6 to +12.9 \\
\hline
\end{tabular}

(1) Monteiro et al., 2007; (2) Rubo and Monteiro, 2010; (3) Percy, 2014; (4) Oliveira, 2013; (5) Baia, 2013.

Monteiro et al.(2007) suggest that despite the differences in the values, the overall isotope compositions in the various deposits are close to the predicted ones for initial metalliferous fluids and suggested involvement of similar metalliferous fluids in the genesis of all these deposits.

The $\delta^{13} \mathrm{C}$ values in gangue carbonate from the willemitic deposits are characteristically positive ( +0.1 to $+1.3 \%$; Monteiro et al., 2006; Percy, 2014). Relatively low $\delta^{13} \mathrm{C}$ values (down to $-6.0 \%$ ) are a common characteristic from the sulfide-rich deposits and occurences from the Vazante Group as well as the sulfide occurrences within the willemitic deposits of Vazante and Vazante Northern Extension (Baia, 2013; Monteiro et al., 1999; Monteiro et al., 2006; Percy, 2014; Rubo and Monteiro, 2010). This implies that descendent fluids could have interacted with carbonaceous phyllite, providing lighter carbon content.

\subsection{SAMPLING AND METHODS}

A representative drill core (VZVG003: from $48.25 \mathrm{~m}$ to $620.96 \mathrm{~m}$ ) with remarkable post-sedimentary features and various stages and styles of hydrothermal alteration and two mineralized zones was selected for the detailed study (Fig. 2.3). The core samples were described in collaboration with the Votorantim Metais S.A. exploration team. One hundred and thirty one core samples were selected this study, including fluid-rock interaction features from the earliest to the latest hydrothermal episodes. Unaltered samples were also collected for comparison. A subset of forty six samples with relevant hydrothermal features and cross-cutting relationships were selected for detailed mineralogical studies, and nine samples for fluid inclusion studies, although workable fluid inclusions were only identified in five samples.

Mineral chemistry analyses were determined using a JEOL JXA 8900RL model electronic microprobe of the Microscopy and Microanalysis Laboratory of the Geosciences Institute of the University of Brasília. These analyses were conducted under a $15 \mathrm{kV}$ tension, $20 \mathrm{nA}$ current and $1 \mu \mathrm{m}$ diameter beam. The standards and detection limits for the elements are summarized in Table 1.5. 
Stable isotope analyses were conducted in the various generations of dolomite. The selected grain was drilled from the thin section cutbacks in order to constrain the paragenetic relationship of the sampled mineral phase. The samples were pulverized by a carbide tungsten dental drill with Ni coating and conical shape of $0.40 \mathrm{~mm}$ minimal cutting diameter. For carbon and oxygen stable isotope analysis, $300 \mu \mathrm{g}$ were sampled. The $\mathrm{CO}_{2}$ extraction was done through a reaction with $\mathrm{H}_{3} \mathrm{PO}_{4}$ at $72^{\circ} \mathrm{C}$ after the cleaning of the recipient by He flux. Determination of $\delta^{13} \mathrm{C}$ and $\delta^{18} \mathrm{O}$ in dolomite was accomplished in the Geochronologic, Geodynamic and Environmental Studies Laboratory of University of Brasília. It was through a Thermo Scientific Gas Bench II on-line gas preparation and introduction system. This system works with a continuous flux coupled to a Thermo Scientific DELTA V Isotope Ratio mass spectrometer. The precision is $0.05 \%$ for $\delta^{13} \mathrm{C}$ and $0.10 \%$ for $\delta^{18} \mathrm{O}$ and the results are reported as $\delta^{13} \mathrm{C} \mathrm{PDB}$ (Pee Dee Belemnite) values and $\delta^{18} \mathrm{O}$ PDB and $\delta^{18} \mathrm{O}$ SMOW (Standard Mean Ocean Water) values.

Whole rock geochemical analyses were performed by ALS Global. Samples were pulverized in a widia pan mill and between each pulverization the pan was decontaminated through cleaning and pulverization of quartz. Major elements were analyzed through X-ray fluorescence (XRF) of whole rock by fusion and LOI through furnace and XRF. The elements $\mathrm{Ba}, \mathrm{Ce}, \mathrm{Cr}, \mathrm{Cs}$, Dy, Er, Eu, Ga, Gd, Hf, Ho, La, Lu, Nb, Nd, Pr, Rb, Sm, Sn, Sr, Ta, Tb, Th, Tm, $\mathrm{U}, \mathrm{V}, \mathrm{W}, \mathrm{Y}, \mathrm{Yb}$, and $\mathrm{Zr}$ were analyzed through fused bead, acid digestions (nitric, hydrochloric, and hydrofluoric acids), and inductively coupled plasma - mass spectroscopy (ICP-MS) finish. The elements $\mathrm{Ag}, \mathrm{Cd}, \mathrm{Co}, \mathrm{Cu}, \mathrm{Li}, \mathrm{Mo}, \mathrm{Ni}, \mathrm{Pb}, \mathrm{Sc}$, and $\mathrm{Zn}$ were analyzed through four acid digestion and inductively coupled plasma - atomic emission spectroscopy (ICP-AES) finish. The elements $\mathrm{As}, \mathrm{Bi}, \mathrm{Hg}$, In, $\mathrm{Re}, \mathrm{Sb}, \mathrm{Sc}, \mathrm{Te}$, and $\mathrm{Tl}$ were analyzed through aqua regia digestion and ICP-MS finish. Total carbon and sulfur were analyzed through Leco furnace. Samples with $\mathrm{Zn}$ contents over $10.000 \mathrm{ppm}$ were analyzed by four acid digestion with atomic absortion spectroscopy (AAS) finish and contents over 30\% were analyzed through high-precision classical titration.

\subsection{THE HYDROTHERMAL ALTERATIONS IN THE SERRA DO POÇO VERDE FORMATION IN BARREN AND MINERALIZED ZONES}

\subsubsection{Least altered rocks}

In the VZVG003 drill core samples (figs. 2.4 A-F), the Upper Moro do Pinheiro (462 m to $620 \mathrm{~m}$ of the drill hole) is composed mainly of gray to dark gray laminated to oncolytic dolomite-mudstone to dolomite-microsparstone, with bird's eyes, cyanobacteria mats disturbed by syn-sedimentary faults, and fenestrae porosity (figs. 2.4-D,E,F and 2.5-A,B). There are metric lenses of massif to laminated, cream to pink dolomite-mudstone to dolomitemicrosparstone interbedded with dark gray carbonaceous phyllite. Textural evidences such as bird's eyes structures and fenestrae porosity suggest that the original porosity was high. The dolostones are composed by cyanobacteria mats interbedded with detritic textured strata. These features indicate shallow water and high energy depositional environment, probably shallow subtidal. Microsparitic texture is derived from partial to total obliteration of the primary texture (and sometimes structures) by recrystallization. The contact with the overlain unit is gradational.

The Lower Pamplona Member (from $46 \mathrm{~m}$ to $462 \mathrm{~m}$ ) is represented by gray and pink dolomite-mudstone to dolomite-microsparstone (Fig. 2.4-A,B,C). They commonly comprise cyanobacteria mats, partially obliterated oncolytic texture with pronounced porosity (Fig. 2.5- 
C), bird's eyes, fenestrae and massif structures, and autigenic quartz (Fig. 2.5-D). They are interbedded with centimetric to metric, very fine sandstone with carbonate cement commonly represented by nodular accretion (Fig. 2.5-E), and greenish, marooned, and dark gray commonly carbonate- and tourmaline-bearing phyllites layers, and they commonly display S-C mylonitic foliation with quartz sigmoids (Fig. 2.5-F).
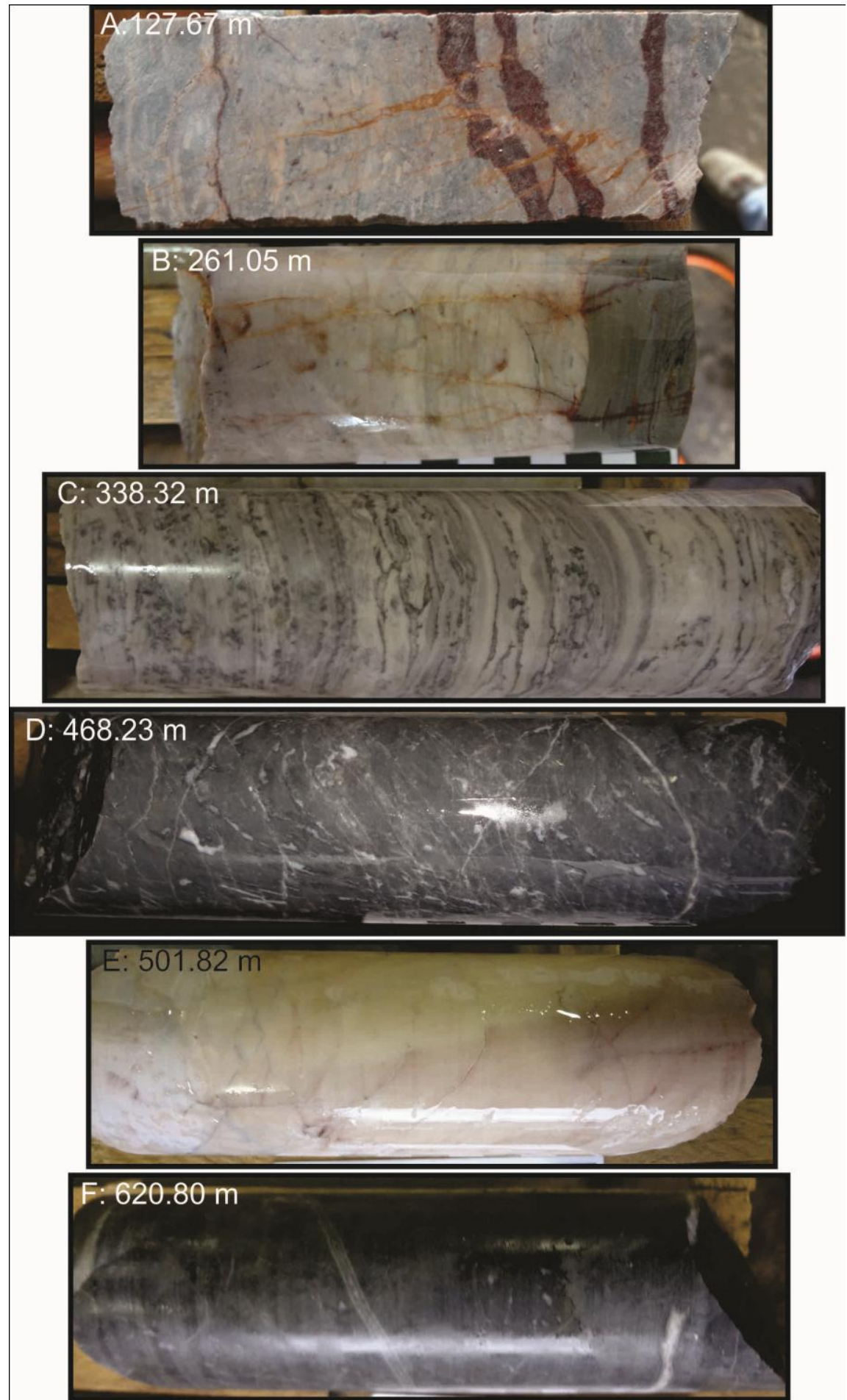

Fig. 2.4. Least altered rocks from the drill core VZVG003. Lower Pamplona Member samples from A to C: A: laminated gray dolomite cross-cut by ore stage alteration related red dolomite veinlets; B: laminated pink to gray dolomite in contact with green phyllite at the bottom cross-cut by tiny ore related red dolomite veinlets; C: laminated gray dolomite with bird's-eyes. Upper Morro do Pinheiro Member samples from D to F: D: dark gray massif dolomite with bird's eyes cross-cut by white carbonate veinlets; E: massif dolomite, pinkish along fractures; F: laminated dark gray dolomite cross-cut by white carbonate veinlets. Drill core width is $4.8 \mathrm{~cm}$; values in the left corner represent depth in the drill hole. 


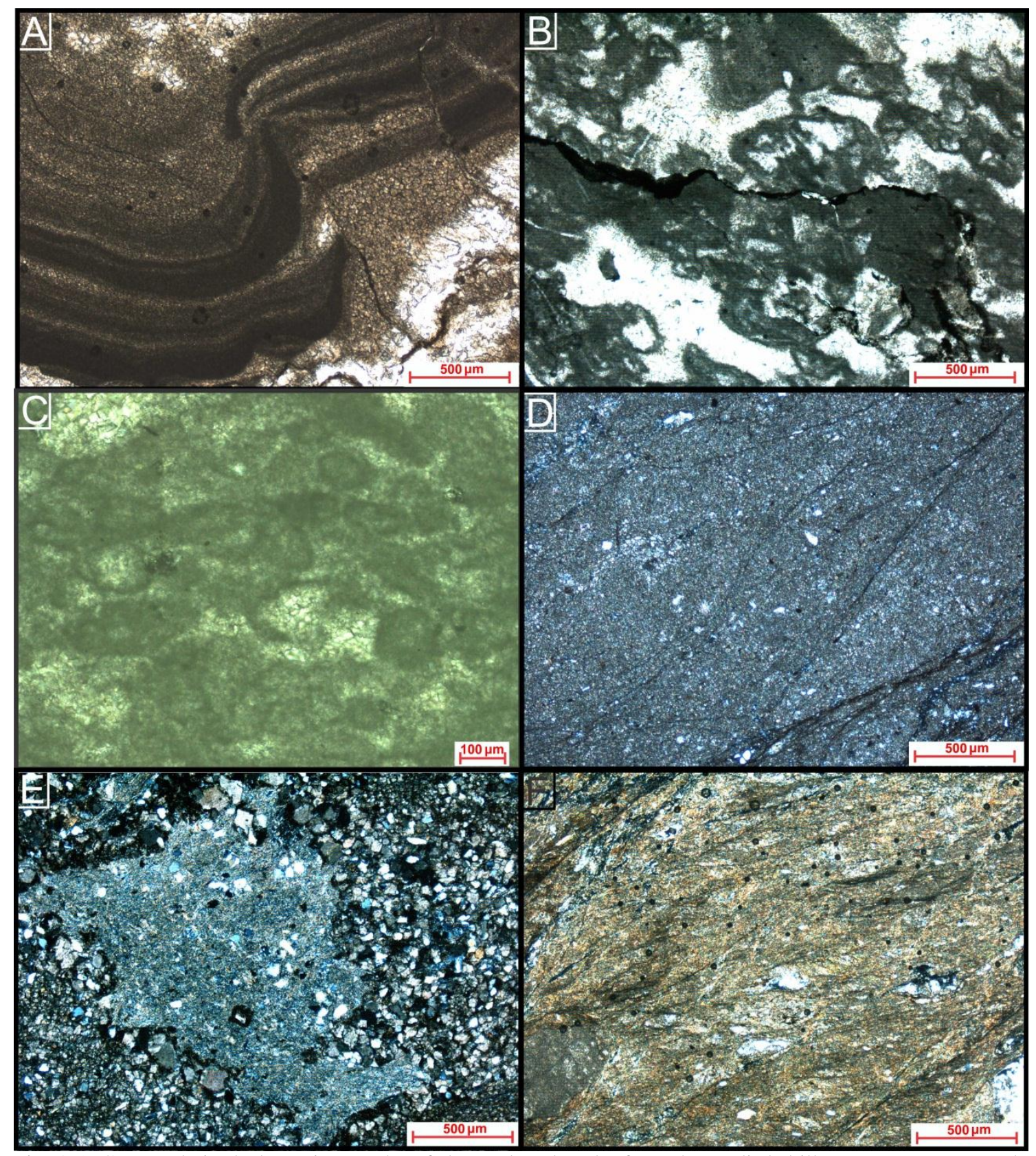

Fig. 2.5. Cross polarized photomicrographs of the unaltered rocks from the studied drill core. Upper Morro do Pinheiro Member samples from A to B: A: cyanobacteria mats disturbed by syn-sedimentary fault with late sparitic cementation (sample $603.32 \mathrm{~m}$ ). B: fenestrae porosity in dolomudstone filled by sparitic cementation (sample 603.32 $\mathrm{m}$ ); Lower Pamplona Member samples from C to F. C: oncolytic texture partially obliterated by recrystallization and sparitic cementation (sample $261.05 \mathrm{~m}$ ); D: massif structure dolostone with microsparitic texture and few autigenic quartz grains with incipient S-C mylonitic foliation (sample $416.09 \mathrm{~m}$ ); E: carbonate nodule and carbonate cement between very fine sandstone quartz grains (sample $84.67 \mathrm{~m}$ ); and F.. phyllite with $\mathrm{S}-\mathrm{C}$ mylonitic foliation with quartz sigmoids (sample $439.61 \mathrm{~m}$ ).

\subsubsection{Diagenetic and hydrothermal alterations}

Based upon detailed petrographic investigation, six types of hydrothermal alterations were recognized in the drill-cores (figs. 2.6 and 2.7) and they are described below: 


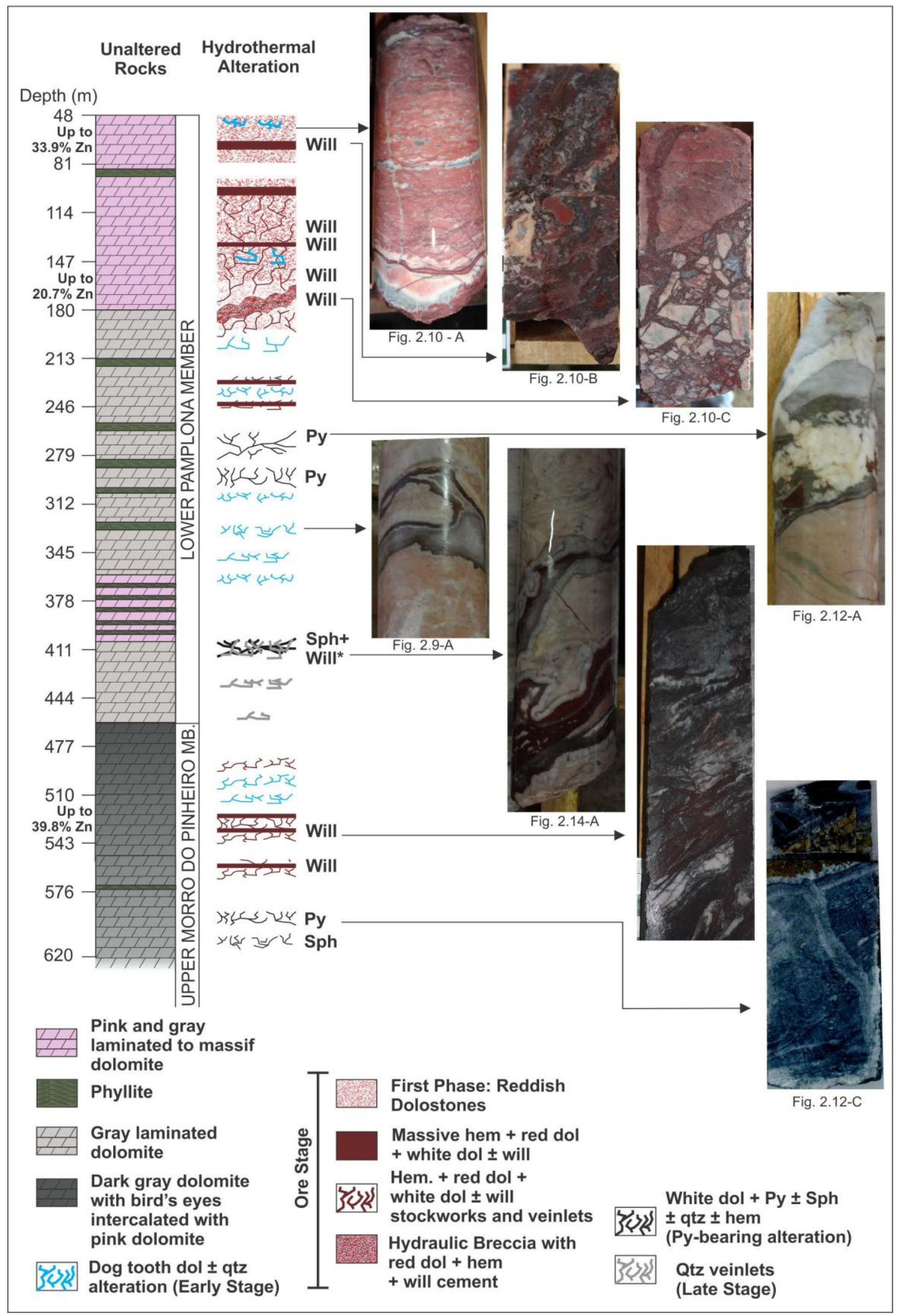

Fig. 2.6. Rock units and various styles of alteration documented along the drill core VZVG003. Two mineralized levels: one in the Upper Morro do Pinheiro Member between 523 and $565 \mathrm{~m}$ at $7.87 \% \mathrm{Zn}$, with up to $39.8 \% \mathrm{Zn}$; other one in the Lower Pamplona Member between 62 and $75 \mathrm{~m}$ at $7.38 \% \mathrm{Zn}$, with up to $33.9 \% \mathrm{Zn}$. In the Lower Pamplona there is na occurrence of hydraulic breccia with up to $20.7 \%$ Zn.For drill core location see Figure 2.3. Abbreviations: Dol = dolomite; Fig. = figure; Hem = hematite; $\mathrm{Py}=$ pyrite; $\mathrm{Sph}=$ Sphalerite; Will = willemite; Will ${ }^{*}$ $=$ Late Stage willemite, product of reaction between sphalerite from the galena-bearing alteration and quartz from the Late Stage. 


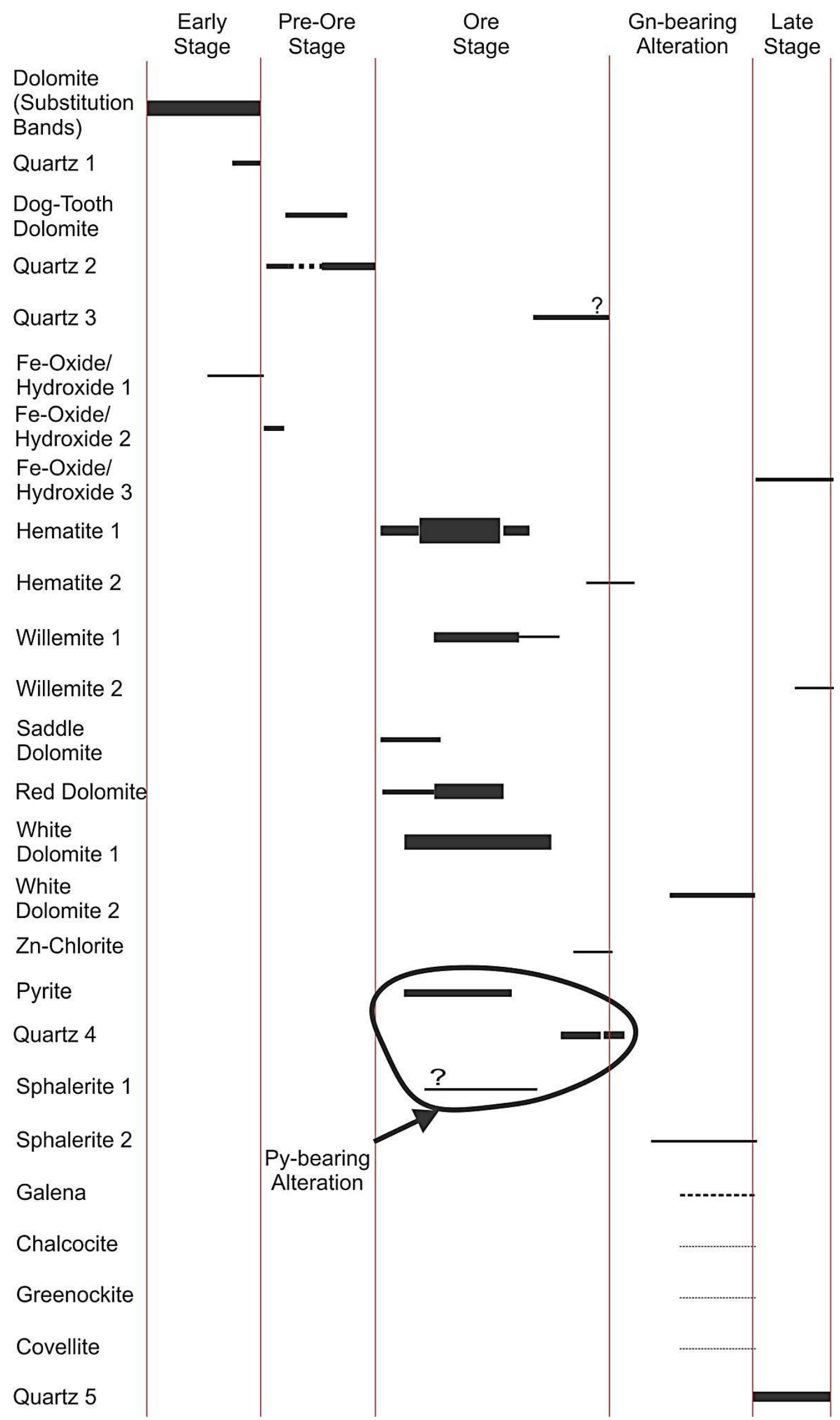

Fig. 2.7. Paragenetic sequence of the hydrothermal alterations identified in the Upper Morro do Pinheiro and Lower Pamplona members od the Vazante Group (drill core VZVG003). Py = pyrite; Gn = galena. 
Early Stage: This alteration is marked by the development of millimetric substitution bands of white to pale yellow, spar dolomite (Fig. 2.8) and in few places in association with very fine quartz and very fine Fe-oxide/hydroxide. Quartz is preferentially found in the central filling portions of these substitution features, suggesting being a late mineral phase in this alteration. These bands are preferentially parallel to sub-parallel to the primary lamination. They show a gradual contact to the framework, as a progressive transition from coarse sparitic texture from the substitution bands to microsparitic/micritic texture from the host dolomite. This style of alteration is also represented by nodules of more irregular shapes that cross-cut the primary lamination and normally are connected with the more copious substitution bands. In some levels there is a clear obliteration of the primary lamination by the pervasive development of spar dolomite. This style of alteration is widespread in the entire length of the studied drill hole and commonly observed in outcrops of the Upper Morro do Pinheiro and Lower Pamplona members.

Pre-Ore Stage: This alteration is composed of dog-tooth and blocky dolomite, quartz (Figs. 2.9-A,B) and commonly associated with very fine Fe-oxide/hydroxide filling_cavities and voids between clasts of collapse breccias from the Upper Morro do Pinheiro and Lower Pamplona Members. Dog-tooth dolomite occurs preferentially on the borders of the cavities and is overgrowth by quartz in the central portions (Fig. 2.9-C). In few occurrences the dolomite from this alteration style also displays brecciated texture overgrown by other generations of dolomite (Fig. 2.9-D). This suggests that dissolution processes were coeval to the precipitation of these mineral phases. When present, Fe-oxide/hydroxide occurs associated with both quartz and dolomite (figs. 2.9-C,D).

Ore Stage: This alteration comprises reddish to white dolomite, hematite, willemite, quartz and zincian chlorite and is well developed in the Lower Pamplona and Upper Moro do Pinheiro members (48 to $210 \mathrm{~m}$ and 480 to $570 \mathrm{~m}$, respectively; Fig. 2.6). It exhibits four phases of mineral precipitation: the first phase shows the widest reddish alteration halo with disseminated hematite and red dolomite (Fig. 2.10-A), and also veinlets of blocky (Fig. 2.11-A) and saddle (Fig. 2.11-B) ocher yellowish dolomite with hematite micro-inclusions and it overprints the pre-ore stage alteration (Fig. 2.10-A). The second phase is the main ore stage, and it is represented by abundant massive red dolomite, stringers of and massive hematite and granular to fibro-radiated willemite as hydrothermal cement more commonly in hydrothermal dissolution breccias (Fig. 2.10-B and Fig. 2.11-C), but also in hydraulic breccias (Fig. 2.10-C restricted to Lower Pamplona Member), veinlets and stockworks (Fig. 2.10-D). The red color of dolomite is due to micro-inclusions of hematite. The third phase is characterized by white dolomite, hematite and traces of willemite that cross-cut the main ore alteration phase (Fig. 2.10-E and figs. 2.11-D,E,F), and usually occur confined to the same major structures (Fig. 2.10-F). The fourth phase comprises zincian chlorite and quartz that fill corroded zones and voids in the previous phases (Fig. 2.11-E).

Sulfide Alterations: There are two types of sulfide alterations: One composed of pyrite, sphalerite, fine hematite, white dolomite and quartz (further referred to as pyrite-bearing alteration) and the other one is characterized by sphalerite, white dolomite and traces of galena, chalcocite, greenockite and covellite (further referred to as galena-bearing alteration).

The pyrite-bearing alteration is found in veinlets, and stockworks in both Upper Morro do Pinheiro and Lower Pamplona members, in levels immediately below the willemitic mineralized zones. It is characterized by fine quartz and hematite that occur preferentially on the borders of the veinlets, overgrowth by pyrite, and white dolomite that fills fractures which cross-cut the previous mineral phases (Fig. 2.12-A). There are other occurrences in which quartz and fine hematite are on the borders and are overgrown by white dolomite and massif pyrite 
preferentially in the center of the veinlets. Quartz also occurs in the central portions of the veinlets, but exhibits irregular contacts with dolomite, indicating two generations of quartz (Fig. 2.12-B and figs. 2.13-A,B). Dolomite also forms intergrowths with disseminated sphalerite, pyrite, and quartz with complex texture filling irregular shaped dissolution voids and veins (figs. 2.12-C,D and Fig. 2.13-C). These veins are composed of white dolomite on the borders, varying from fibrous dolomite to blocky dolomite towards the center of the veinlet, with pyrite and quartz films in between dolomite (Fig. 2.13-D). The center of the veins comprises quartz, pyrite and minor dolomite and quartz occurs between pyrite and dolomite, indicating various episodes of carbonate, quartz and sulfide precipitation (Fig. 2.12-C and figs. 2.13-D,E). The cross-cutting relationships evidences between this style of sulfide alteration and the ore stage are scarce. At $267 \mathrm{~m}$ depth in the drill hole, white dolomite, pyrite and minor hematite exhibit ambiguous cross-cutting relationships and diffuse contacts with ocher yellowish saddle dolomite and hematite attributed to the ore stage. White dolomite and quartz cuts ocher saddle dolomite, but is also cut by ocher saddle dolomite, suggesting co-precipitation (Fig. 2.14-A). This sulfide alteration style does not occur in depths shallower than $267.19 \mathrm{~m}$.

The galena-bearing alteration style (Fig. 2.13-F) occurs in the Lower Pamplona Member and it is documented at shallower levels $(180.24 \mathrm{~m})$, where the pyrite-bearing alteration style is not recognized. At this shallower level white dolomite with galena filled dissolution zones in red dolomite from the ore stage (Fig. 2.14-B).

Late Silicification: Quartz and Fe-oxide/hydroxide veinlets cross-cut the sulfide alteration. In some locations, this quartz reacts with sphalerite from the sulfide alteration to produce other generation of willemite, though in trace amounts (Fig. 2.14).

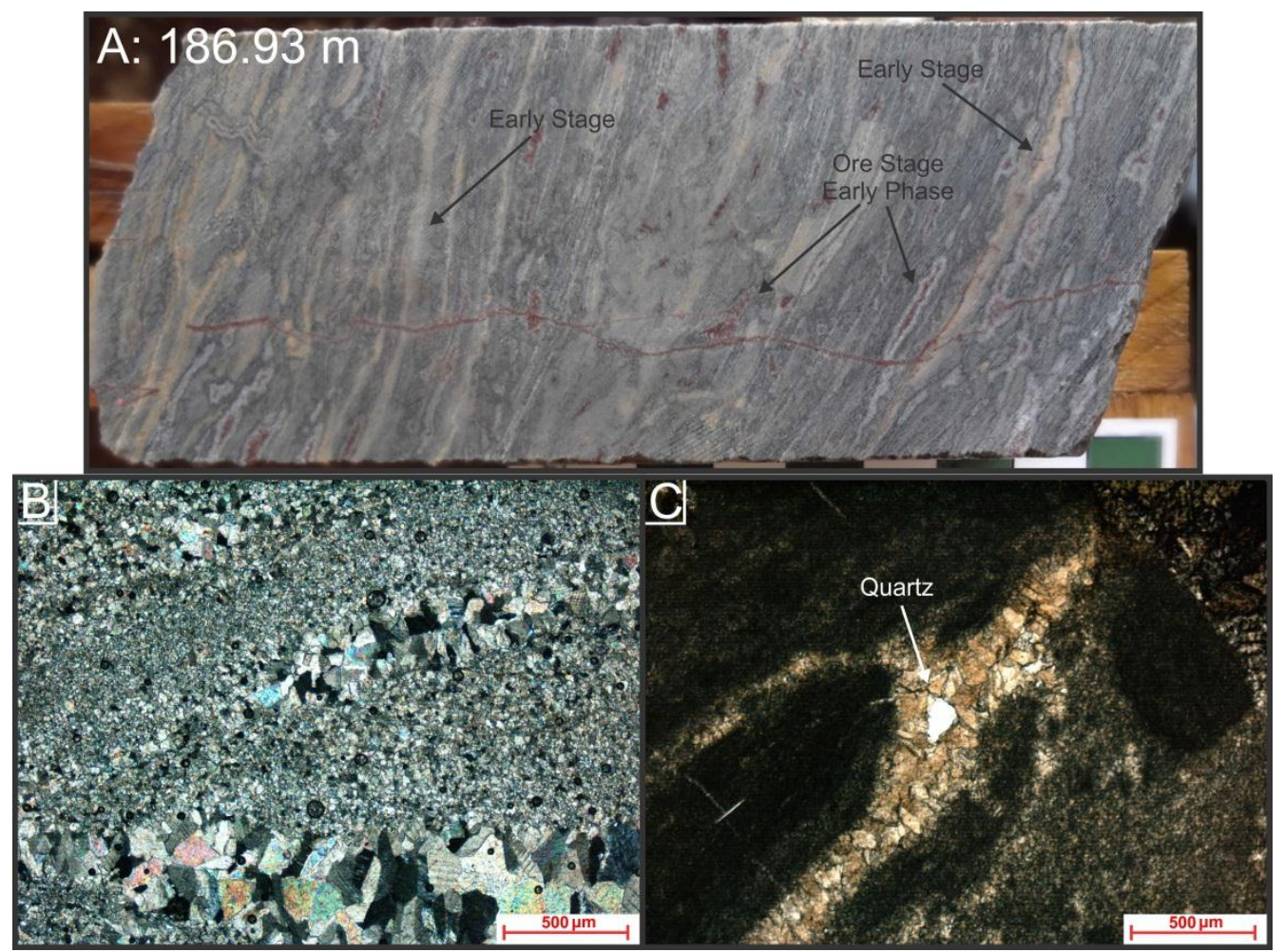

Fig. 2.8. Early Stage alteration in the Lower Pamplona Member. A: substitution bands parallel to sub-parallel to the primary lamination, cross-cut by veinlet and patchy red dolomite substitution nodules from the early phase of the ore stage alteration $(186.93 \mathrm{~m})$. Drill core width is $4.8 \mathrm{~cm}$. B: cross-polarized photomicrograph of coarse dolomite substitution bands parallel to sub-parallel to the primary lamination (sample $275.36 \mathrm{~m}$ ). B: plane-polarized photomicrographs of early dolomite substitution bands with overgrowth by quartz in the central portion of the band $(127.67 \mathrm{~m})$ 

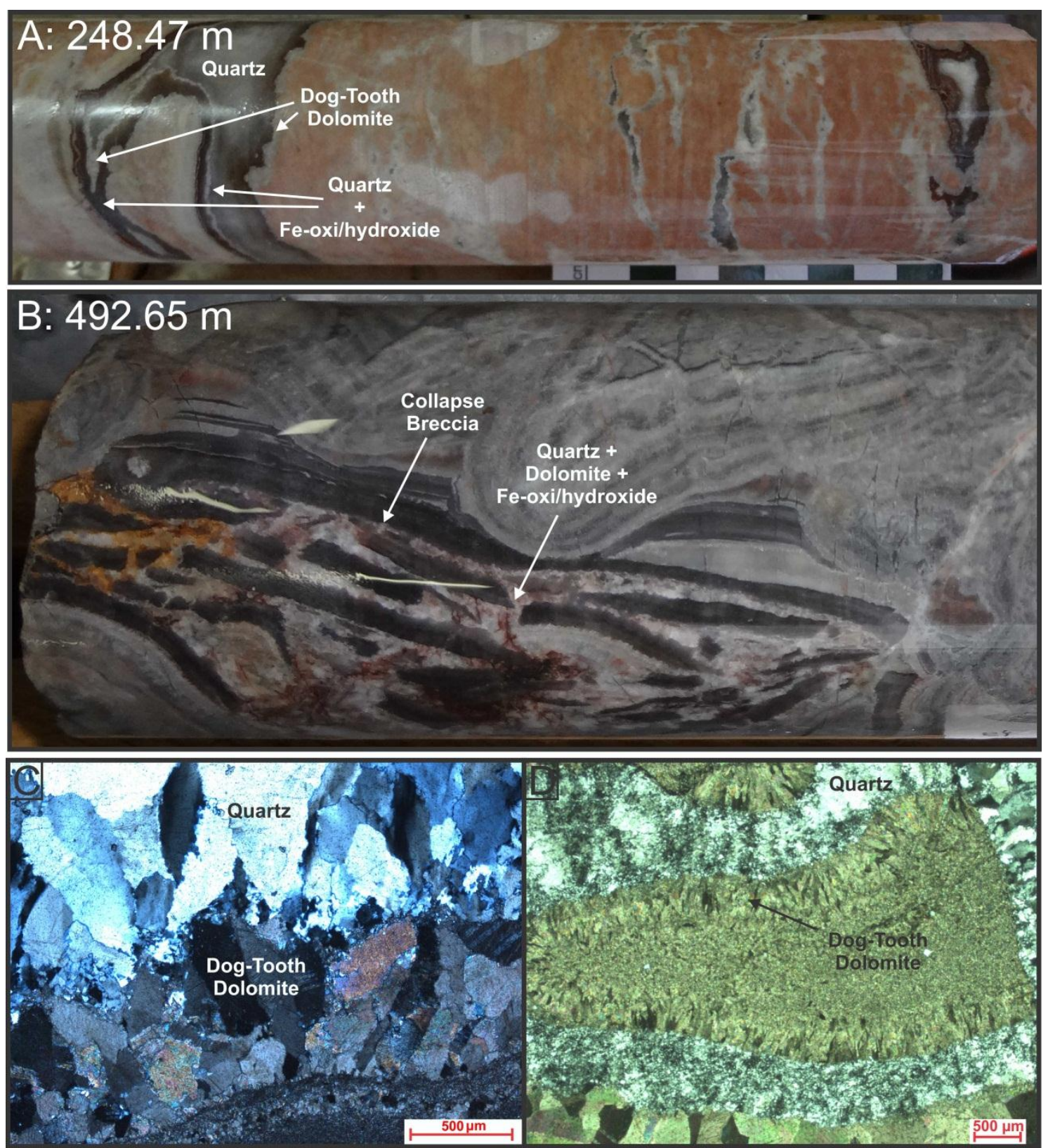

Fig. 2.9. Pre-Ore Stage alteration. Cross polarized microphotographs of core samples from the Lower Pamplona Member. A: drill-core sample with dissolution cavities filled with very fine hematite and quartz on the outermost borders, overgrowth by dog-tooth dolomite and, in the central portions, by quartz $(248.47 \mathrm{~m})$. B: drill core sample with collapse brecciation and hydrothermal cementation with dog-tooth dolomite, quartz and Fe-oxi/hydroxides $(492.65 \mathrm{~m})$. Drill core width is $4.8 \mathrm{~cm}$. C (367.73 m) and D (202,53 m) showing veinlet and stockwork, respectively, with dog-tooth dolomite on the borders and quartz in the central portion;. 

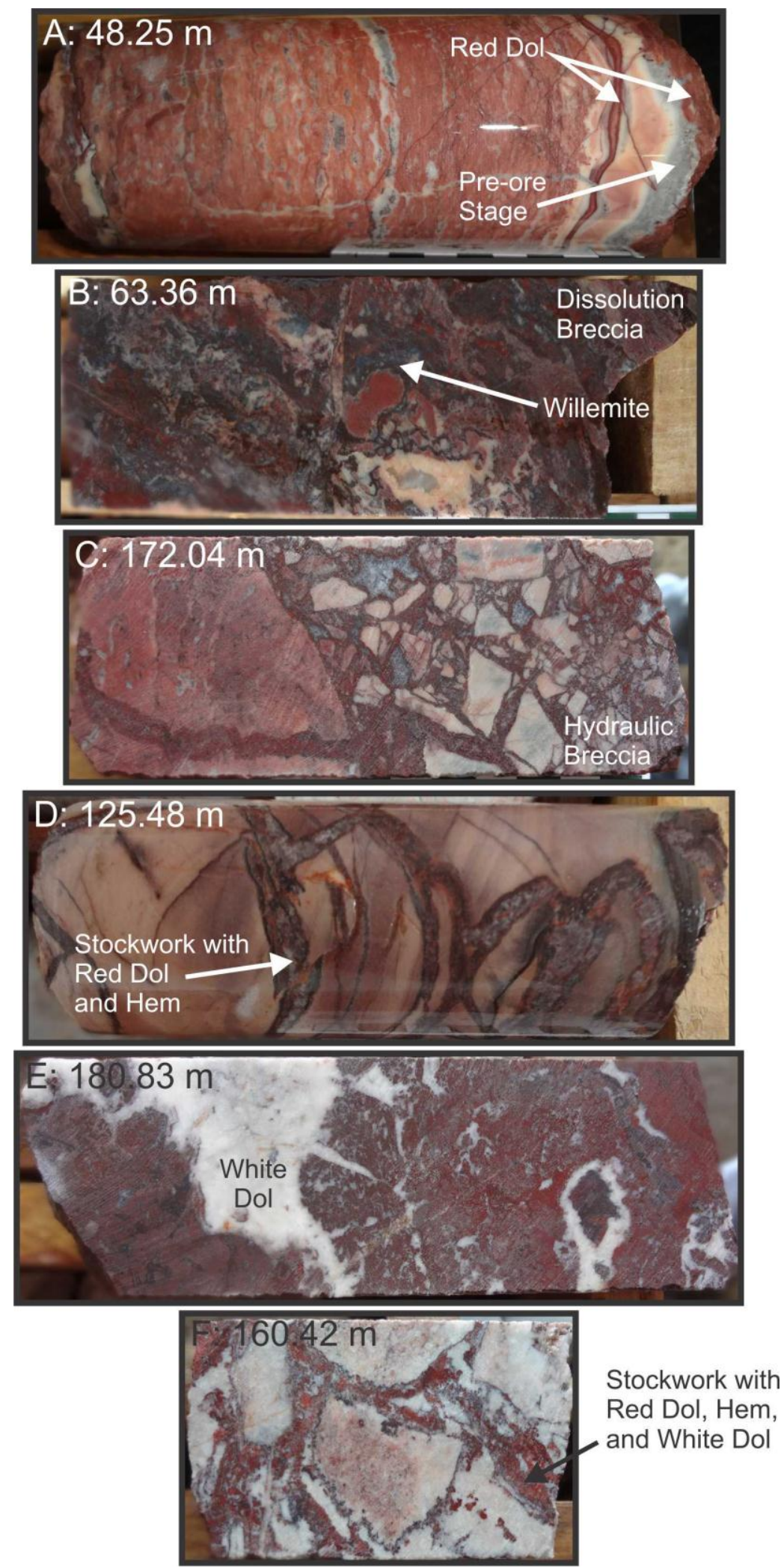

Fig. 2.10. Drill core samples showing the various events related to the ore stage alteration in the Lower Pamplona Member. A: First phase is marked by reddish staining of the host rock due to incipient hematite and red dolomite alteration. Note that this feature cross-cut the pre-ore stage dog -tooth dolomite and quartz alteration; B and C: Second phase of alteration, marked by dissolution brecciation and hydraulic brecciation respectively; D: Stockwork of red dolomite and hematite from the second phase; E: Third phase of alteration charecterized by White dolomite overprinting the second phase; F: Stockwork with red dolomite, hematite and white dolomite, suggesting progressive transition of the second to the third phase of hydrothermal alteration. Drill core width is $4.8 \mathrm{~cm}$. Dol = dolomite; Hem $=$ hematite; $\mathrm{Qtz}=$ quartz. 


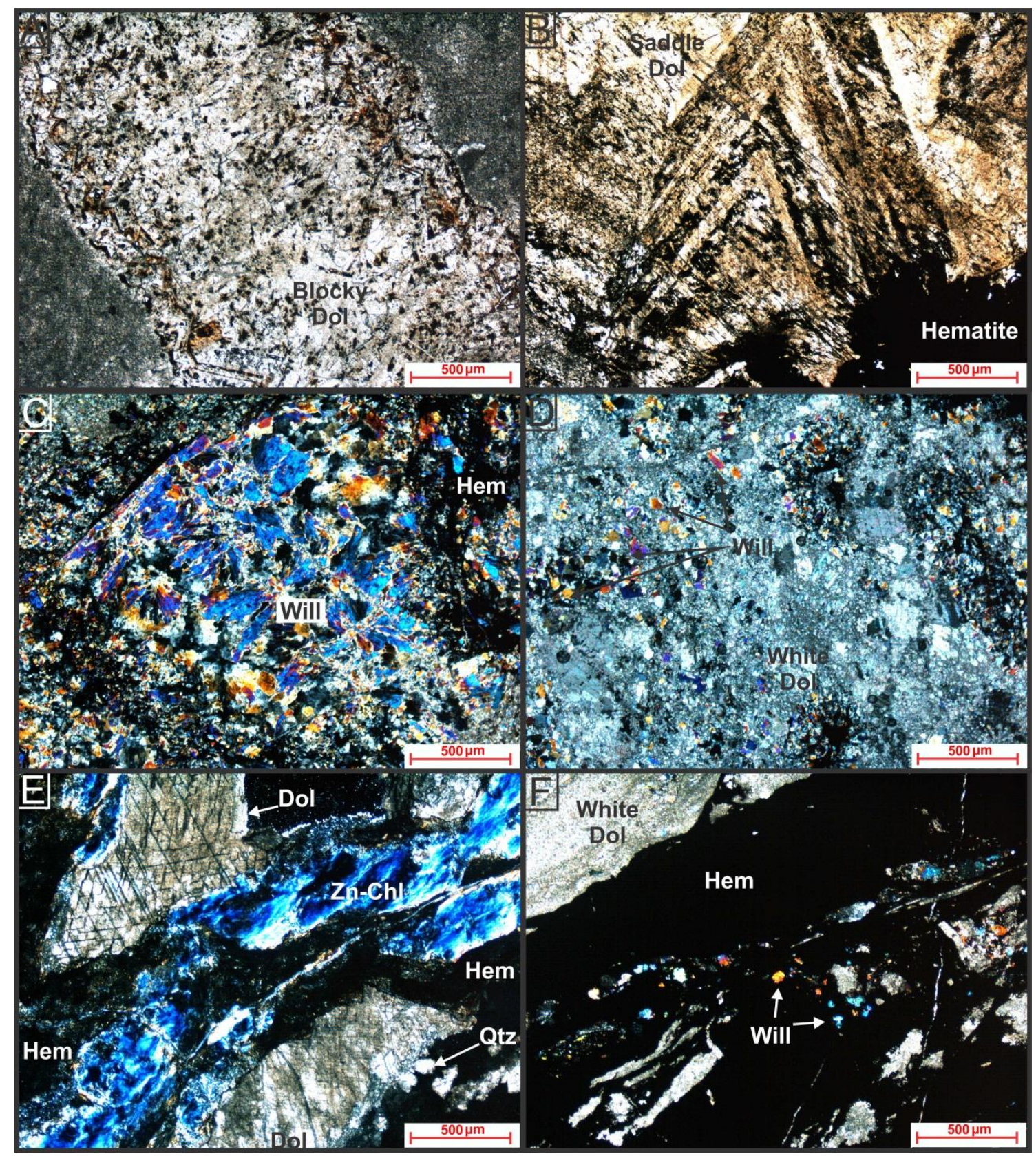

Fig. 2.11. Ore stage alteration photomicrographs. Lower Pamplona Member samples from A to E: A and B (plane polarized): First phase of alteration veinlets with blocky dolomite $(267.19 \mathrm{~m})$ and saddle dolomite $(127.67 \mathrm{~m})$, respectively, both showing abundant micro-inclusions of hematite. $\mathrm{C}$ to $\mathrm{F}$ microphotographs under cross polarized light. C: Fibro-radiated willemite and hematite of the second phase of alteration (63.36 m); D: Granular willemite and hematite from the second phase of alteration substituted by white dolomite of the third phase of alteration $(63.36 \mathrm{~m})$; E: massive hematite from the second phase substituted by white dolomite from the third phase which in turn is crosscut by zincian chlorite and quartz from the fourth phase $(160.42 \mathrm{~m})$; F: Stringers of and massive veins with hematite overgrowth by white dolomite and willemite from the third phase in the Upper Morro do Pinheiro Member (525.41 m). Dol = dolomite; Hem = hematite; $\mathrm{Qtz}=$ quartz; Will = willemite; $\mathrm{Zn}-\mathrm{Chl}=\mathrm{Zn}$-chlorite . 

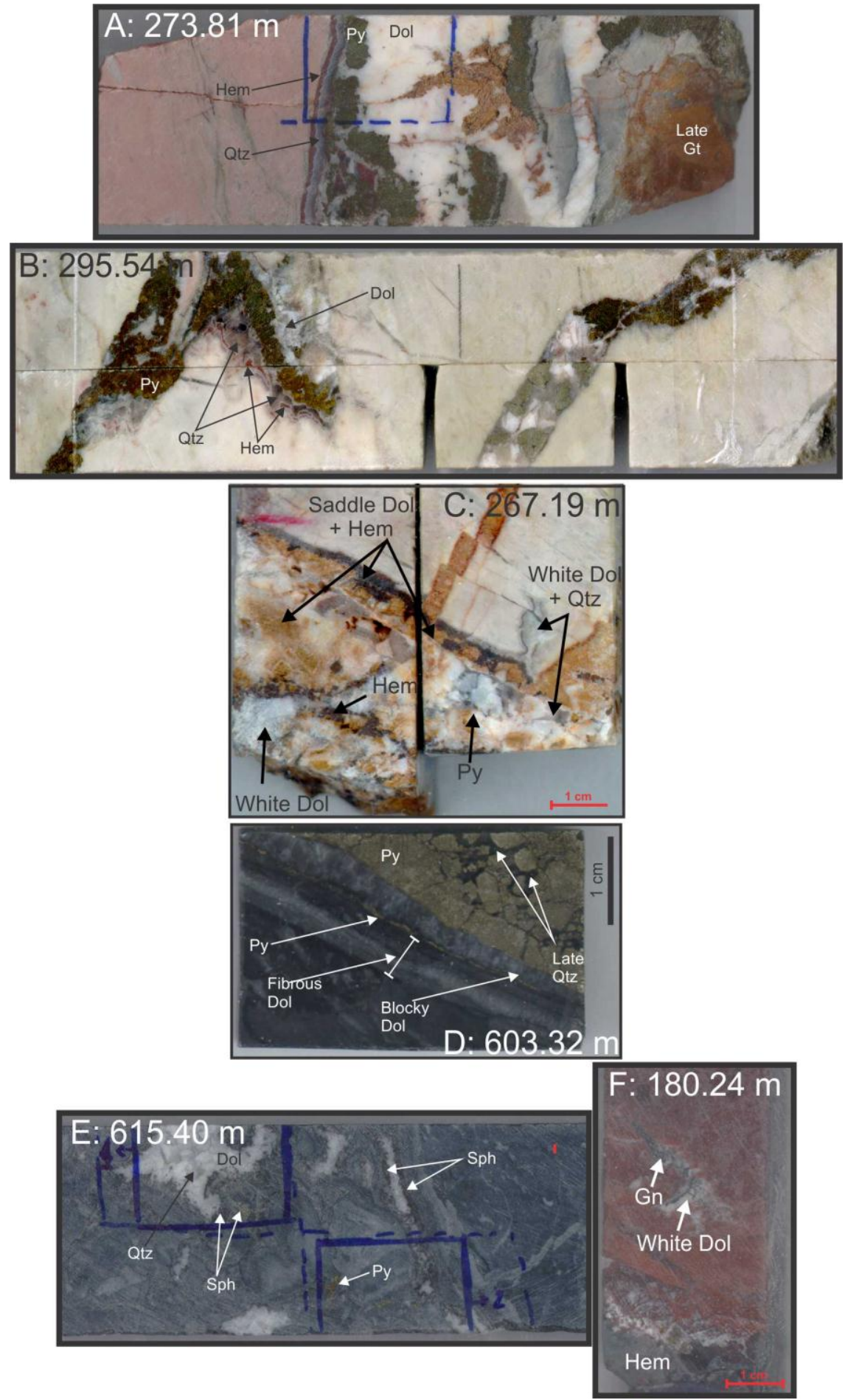

Fig. 2.12. Pyrite-bearing alteration: Lower Pamplona Member drill core samples from A to C: A: Fine hematite and quartz occur on the borders of veinlet and with overgrowth of pyrite which is also overgrown by white dolomite; B: Fine hematite and quartz on the borders of the veinlet overgrown by white dolomite and pyrite. C: white dolomite, hematite, quartz and pyrite show complex intergrowth texture with ocher yellow saddle dolomite from the ore stage. Upper Morro do Pinheiro Member drill core samples from D to E: D: Various episodes of open-filling where fibrous dolomite occurs on the borders of the veinlet. Towards the center of the veinlet there is a film of pyrite overgrown by blocky dolomite and the center of the veinlet comprises pyrite overgrown by quartz; E: Complex substitution texture of the host dolostone by sphalerite, pyrite and white dolomite. Blue marks indicate zones where polished thin sections were cut. F: white dolomite and galena from the galena-bearing alteration cross-cut red dolomite and hematite from the ore stage alteration. Dol = dolomite; $\mathrm{Gn}=$ galena; Hem = hematite; $\mathrm{Py}=$ pyrite; $\mathrm{Qtz}=$ quartz; $\mathrm{Sph}=$ sphalerite. 


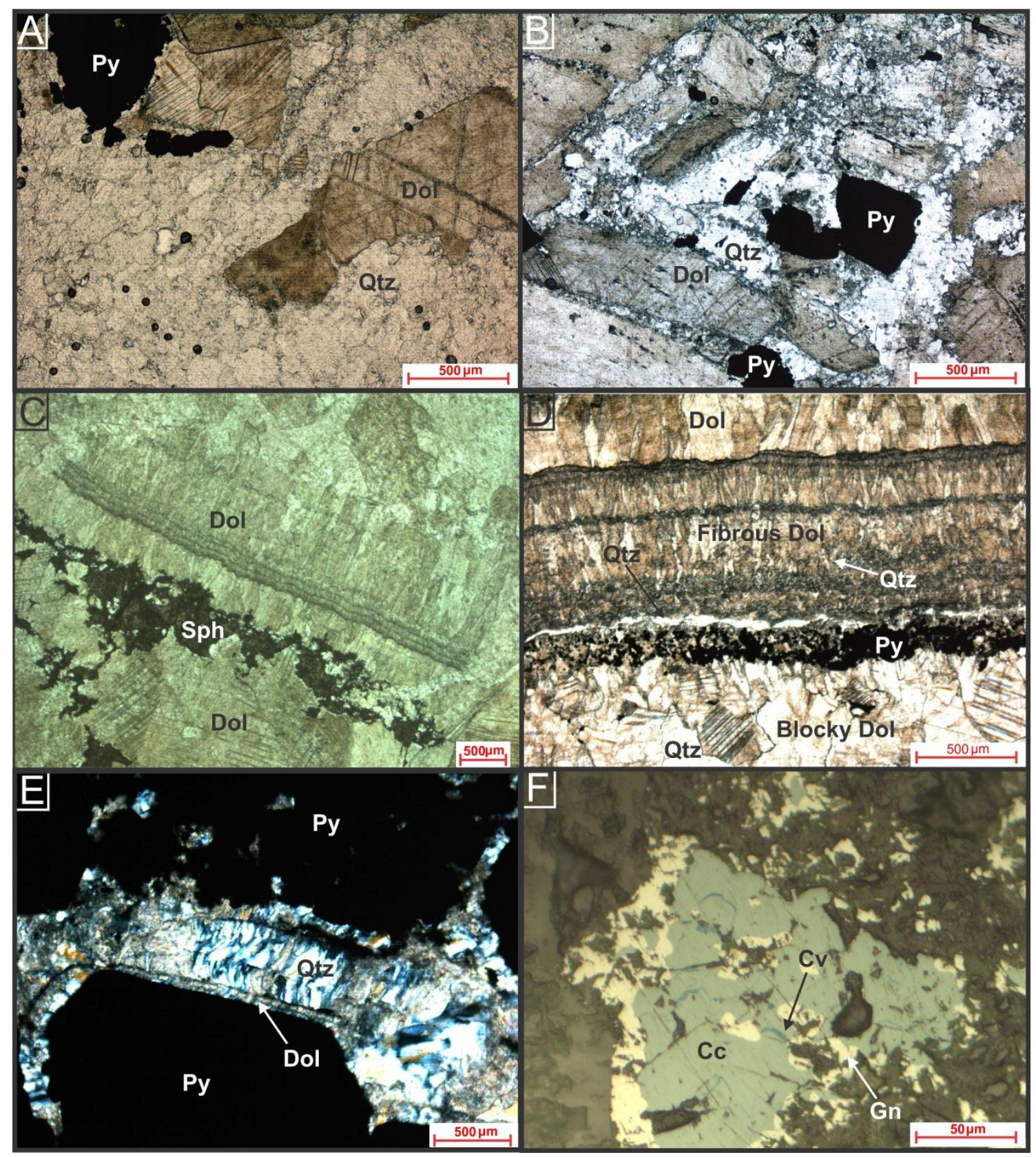

Fig. 2.13. Pyrite-bearing alteration: Lower Pamplona samples are A $(295.54 \mathrm{~m})$ and B $(267.19 \mathrm{~m})$ : Plane polarized photomicrographs of pyrite associated to white dolomite, which is substituted by quartz. Upper Morro do Pinheiro Member samples from C to E: C: Plane polarized photomicrograph of sphalerite associated with dolomite $(615.40$ $\mathrm{m}$ ); D: Plane polarized photomicrography of the transition of fibrous dolomite to blocky dolomite and quartz towards the center of the veinlet with a pyrite and quartz films in between $(603.32 \mathrm{~m})$; E: Quartz showing deformed antitaxial open-filling texture between pyrite crystals $(603.32 \mathrm{~m})$. Galena-bearing alteration: F: Occurrence of intergrowth galena, chalcocite and covellite filling late dissolution zones in the altered dolomite $(411.13 \mathrm{~m}) . \mathrm{Cc}=\mathrm{chalcocite} ; \mathrm{Cv}$ $=$ covellite Dol = dolomite $\mathrm{Gn}=$ galena; $\mathrm{Qtz}=$ quartz; $\mathrm{Py}=$ pyrite $\mathrm{Sph}=$ sphalerite. 


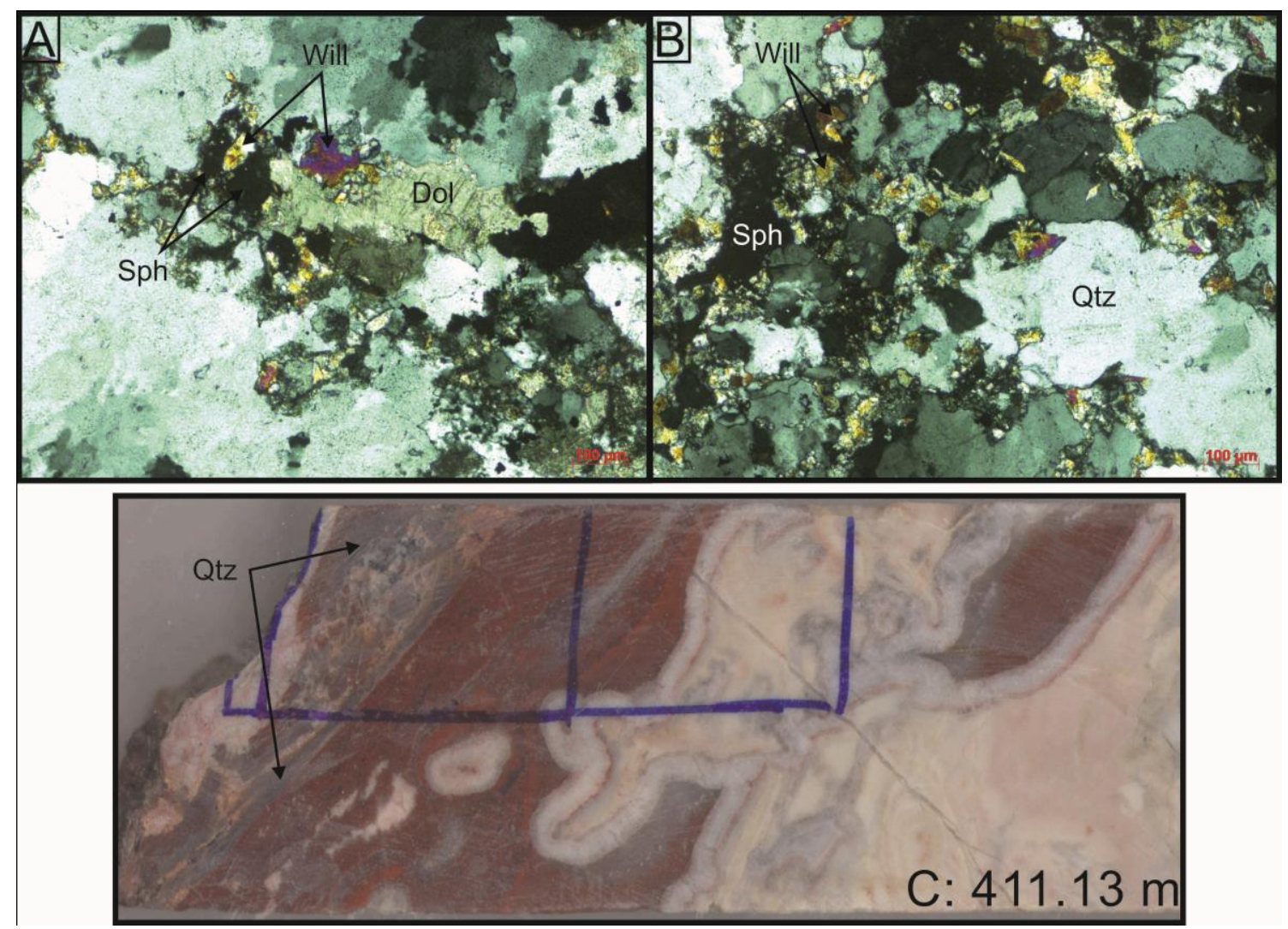

Fig. 2.14. Late silicification. A and B $(411.13 \mathrm{~m})$ : Cross polarized photomicrographs of late stage quartz and willemite that fills corroded zones in white dolomite from the galena-bearing alteration. C: drill-core sample showing the superposition of the Late Stage alteration over the galena-bearing alteration.

\subsection{MINERAL CHEMISTRY}

\subsubsection{Dolomite}

Electron microprobe analyses were conducted in samples of dolomite from the early stage alteration in the Lower Pamplona Member (samples $127.67 \mathrm{~m}$ and $267.19 \mathrm{~m}$ ), red dolomite from the main ore stage alteration in the Lower Pamplona Member (samples $63.36 \mathrm{~m}$, $127.67 \mathrm{~m}, 160.42 \mathrm{~m}$ and $172.04 \mathrm{~m}$ ) and white dolomite from the pyrite-bearing alteration in the Upper Morro do Pinheiro Member (sample $615.40 \mathrm{~m}$ ) in the Lower Pamplona Member close to the ore stage alteration halo (sample $267.19 \mathrm{~m}$ ) and from the galena-bearing alteration in the Lower Pamplona Member (sample $411.13 \mathrm{~m}$ ). The results are reported in Table 2.4 and plot in Figure 2.15.

The $\mathrm{CaO}$ contents in dolomite from the galena-bearing alteration are higher than in the other alterations (averages of $37.34 \%$ and up to $34.39 \%$, respectively; Table 2.4; Fig. 2.15). The content of $\mathrm{MgO}$ does not show considerable variation, except to the main ore stage red dolomite (22.64\% average), which is depleted in relation to the other alterations (Table 2.4; Fig. 2.15). The FeO content is more elevated in dolomite from the pyrite-bearing sulfide alteration in the Upper Morro do Pinheiro Member and in the galena-bearing alteration in the Lower Pamplona Member (1.39\% and $2.47 \%$ averages, respectively). The average $\mathrm{Zn}$ content in red dolomite from the main ore stage is higher than from the other alteration stages $(0.975 \%$ and up to $0.342 \%$ averages, respectively), however there are some very high outlier values from the pyrite-bearing alteration in the Upper Morro do Pinheiro Member (up to 7.622\%). The Cd content is also higher in red dolomite from the main ore stage $(0.024 \%$ average $)$, although there 
are appreciable $\mathrm{Cd}$ contents in dolomite from the pyrite-bearing alteration in the Upper Morro do Pinheiro and Lower Pamplona members (up to 0.047\%). The content of $\mathrm{MnO}$ and $\mathrm{Sb}$ is slightly higher in red dolomite from the main ore stage and from the pyrite-bearing alteration in the Upper Moro do Pinheiro and galena-bearing alteration in the Lower Pamplona Member. Dolomite from the pyrite-bearing alteration in the Lower Pamplona Member close to the ore stage alteration halo is characterized by low metal content.

Table 2.4. Compositional range and average composition in (wt. \%) of different varieties of dolomite from the various alteration styles.

\begin{tabular}{|c|c|c|c|c|c|c|c|c|c|c|c|c|c|c|c|}
\hline \multicolumn{16}{|c|}{ Dolomite } \\
\hline & \multicolumn{3}{|c|}{ Early Stage $(n=13)$} & \multicolumn{3}{|c|}{$\begin{array}{l}\text { Ore Stage Red } \\
\text { Dolomite }(n=38)\end{array}$} & \multicolumn{3}{|c|}{$\begin{array}{l}\text { Py-Bearing Alt. } \\
\text { U.M.P. }(n=45)\end{array}$} & \multicolumn{3}{|c|}{$\begin{array}{l}\text { Py-Bearing Alt. } \\
\text { L.P.C.O.S. }(n=13)\end{array}$} & \multicolumn{3}{|c|}{$\begin{array}{l}\text { Gn-Bearing Alt. } \\
\text { L.P. }(n=25)\end{array}$} \\
\hline & Low & High & Mean & Low & High & Mean & Low & High & Mean & Low & High & Mean & Low & High & Mean \\
\hline $\mathrm{CaO}$ & 33.19 & 34.74 & 34.05 & 32.46 & 34.86 & 33.76 & 33.17 & 35.80 & 34.39 & 33.43 & 34.33 & 33.94 & 35.35 & 38.84 & 37.34 \\
\hline $\mathrm{MgO}$ & 22.46 & 34.34 & 23.19 & 20.83 & 24.39 & 22.64 & 22.26 & 24.18 & 23.25 & 22.56 & 23.94 & 23.46 & 22.60 & 24.48 & 23.62 \\
\hline $\mathrm{FeO}$ & 0.079 & 0.804 & 0.302 & * & 1.471 & 0.486 & 0.085 & 3.211 & 1.389 & 0.120 & 0.971 & 0.489 & 1.928 & 3.726 & 2.474 \\
\hline $\mathrm{MnO}$ & * & 0.403 & 0.105 & $*$ & 0.651 & 0.138 & $*$ & 0.371 & 0.107 & 0.023 & 0.184 & 0.096 & $*$ & 0.327 & 0.176 \\
\hline $\mathrm{Zn}$ & $*$ & 0.503 & 0.147 & $*$ & 3.368 & 0.975 & * & 7.622 & 0.342 & * & 0.088 & 0.015 & $*$ & 0.450 & 0.059 \\
\hline $\mathrm{Cd}$ & $*$ & 0.021 & $*$ & $*$ & 0.236 & 0.024 & $*$ & 0.047 & 0.008 & $*$ & 0.025 & 0.005 & * & 0.033 & 0.006 \\
\hline $\mathrm{Pb}$ & $*$ & 0.532 & 0.106 & $*$ & 0.938 & 0.110 & $*$ & 0.124 & 0.010 & * & * & * & $*$ & 0.124 & 0.013 \\
\hline $\mathrm{Sb}$ & $*$ & 0.065 & 0.016 & $*$ & 0.077 & 0.024 & $*$ & 0.071 & 0.037 & $*$ & $*$ & $*$ & * & 0.082 & 0.037 \\
\hline
\end{tabular}

Abbreviations: * = value < 10x detection limit; Alt. = Alteration; $\mathrm{Gn}=$ Galena; Py = Pyrite; U.M.P. = Upper Morro do Pinheiro Member; L.P. $=$ Lower Pamplona Member; L.P.C.O.S. = Lower Pamplona Member Close to the Ore Stage Alteration Halo.
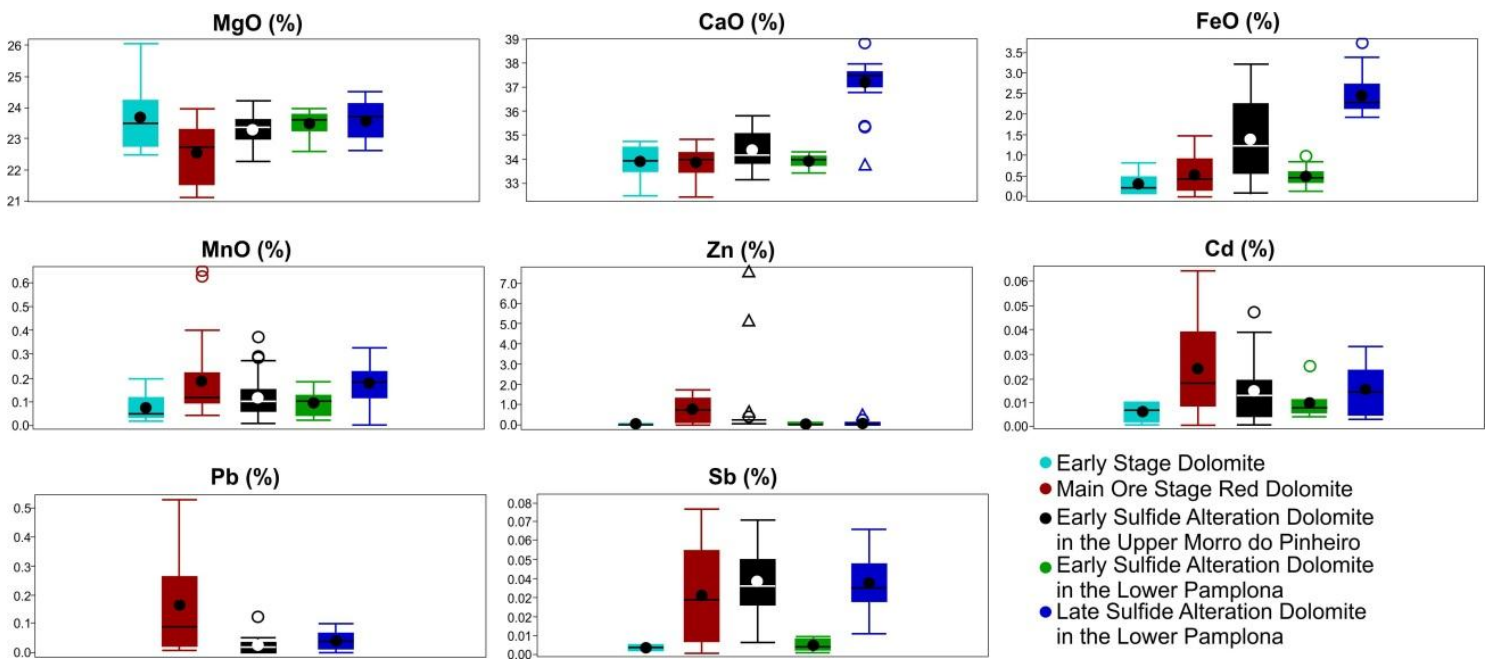

- Early Stage Dolomite

- Main Ore Stage Red Dolomite

- Early Sulfide Alteration Dolomite

in the Upper Morro do Pinheiro

in the Lower Pamplona

in the Lower Pamplona

Fig. 2.15. Box and whisker plots showing the variation in composition of different generations of dolomite.

\subsubsection{Willemite}

Microprobe analyses were performed in willemite from the main ore stage in the Lower Pamplona Member (samples $63.36 \mathrm{~m}, 160.42 \mathrm{~m}$ and $172.04 \mathrm{~m}$ ) and from the late silicification stage in the Lower Pamplona Member (sample $411.13 \mathrm{~m}$ ), which is the product of the reaction between sphalerite from the galena-bearing alteration with quartz from the late stage silicification (Table 2.5; Fig. 2.17). Willemite from the main ore stage show higher average contents of $\mathrm{MnO}$ and $\mathrm{FeO}(0.259 \%$ and $0.035 \%$, respectively) than willemite from the late stage ( $0.071 \%$ and $0.013 \%$, respectively). The average content of $\mathrm{Cd}, \mathrm{Pb}$ and $\mathrm{Sb}$ is higher in willemite from the late silicification than from the main ore stage, although the latter show high outlier values for these three elements (Fig. 2.16). 
Table 2.5. Compositional range and average composition (in wt. \%) of different generations of willemite, sphalerite and pyrite from the various alteration types.

\begin{tabular}{|c|c|c|c|c|c|c|}
\hline \multicolumn{7}{|c|}{ Willemite } \\
\hline & \multicolumn{3}{|c|}{$\begin{array}{l}\text { Main Ore Stage } \\
(n=27)\end{array}$} & \multicolumn{3}{|c|}{ Late Stage $(n=9)$} \\
\hline & Low & High & Mean & Low & High & Mean \\
\hline $\mathrm{SiO}_{2}$ & 21.99 & 26.69 & 25.31 & 25.82 & 27.06 & 26.34 \\
\hline $\mathrm{Zn}$ & 50.31 & 53.38 & 51.91 & 51.94 & 52.73 & 52.19 \\
\hline $\mathrm{FeO}$ & * & 2.549 & 0.259 & $*$ & 0.147 & 0.071 \\
\hline $\mathrm{MnO}$ & * & 0.336 & 0.035 & * & 0.062 & 0.013 \\
\hline $\mathrm{Cd}$ & * & 0.058 & 0.008 & * & 0.026 & 0.007 \\
\hline $\mathrm{Pb}$ & $*$ & 0.324 & 0.031 & $*$ & 0.148 & 0.057 \\
\hline $\mathrm{Sb}$ & * & 0.057 & 0.006 & * & 0.047 & 0.024 \\
\hline \multicolumn{7}{|c|}{ Sphalerite } \\
\hline & \multicolumn{3}{|c|}{$\begin{array}{l}\text { Py-bearing Alteration } \\
\text { U.M.P. }(n=49)\end{array}$} & \multicolumn{3}{|c|}{$\begin{array}{l}\text { Gn-bearing Alteration } \\
\text { L.P. }(n=5)\end{array}$} \\
\hline & Low & High & Mean & Low & High & Mean \\
\hline $\mathrm{S}$ & 31.75 & 33.16 & 32.72 & 32.00 & 32.75 & 32.43 \\
\hline $\mathrm{Zn}$ & 63.00 & 66.35 & 64.81 & 64.07 & 65.89 & 64.92 \\
\hline $\mathrm{Fe}$ & 0.529 & 1.637 & 0.968 & 0.130 & 0.284 & 0.185 \\
\hline $\mathrm{Cu}$ & * & 0.840 & 0.169 & $*$ & 0.186 & 0.091 \\
\hline $\mathrm{Cd}$ & 0.070 & 0.384 & 0.151 & 0.436 & 0.497 & 0.466 \\
\hline $\mathrm{Pb}$ & * & 0.188 & 0.080 & 0.045 & 0.221 & 0.097 \\
\hline \multicolumn{7}{|l|}{ Pyrite } \\
\hline & \multicolumn{3}{|c|}{$\begin{array}{l}\text { Py-bearing Alteration } \\
\text { U.M.P. }(n=25)\end{array}$} & \multicolumn{3}{|c|}{$\begin{array}{l}\text { Py-bearing Alteration } \\
\text { L.P. }(n=16)\end{array}$} \\
\hline & Low & High & Mean & Low & High & Mean \\
\hline$S$ & 52.09 & 54.30 & 53.51 & 53.20 & 53.81 & 53.50 \\
\hline $\mathrm{Fe}$ & 44.21 & 46.94 & 46.26 & 45.58 & 46.81 & 46.25 \\
\hline $\mathrm{Ag}$ & * & 0.027 & 0.004 & $*$ & 0.026 & 0.006 \\
\hline $\mathrm{Cd}$ & * & 0.040 & 0.010 & * & 0.062 & 0.017 \\
\hline $\mathrm{Cu}$ & * & 0.182 & 0.041 & * & 0.400 & 0.035 \\
\hline $\mathrm{Pb}$ & 0.038 & 1.765 & 0.163 & 0.072 & 0.174 & 0.111 \\
\hline $\mathrm{Zn}$ & $*$ & 0.172 & 0.013 & $*$ & 0.032 & 0.006 \\
\hline
\end{tabular}

Abbreviations: $*=$ value $<10 \mathrm{x}$ detection limit; U.M.P. $=$ Upper Morro do Pinheiro Member; L.P. = Lower Pamplona Member;
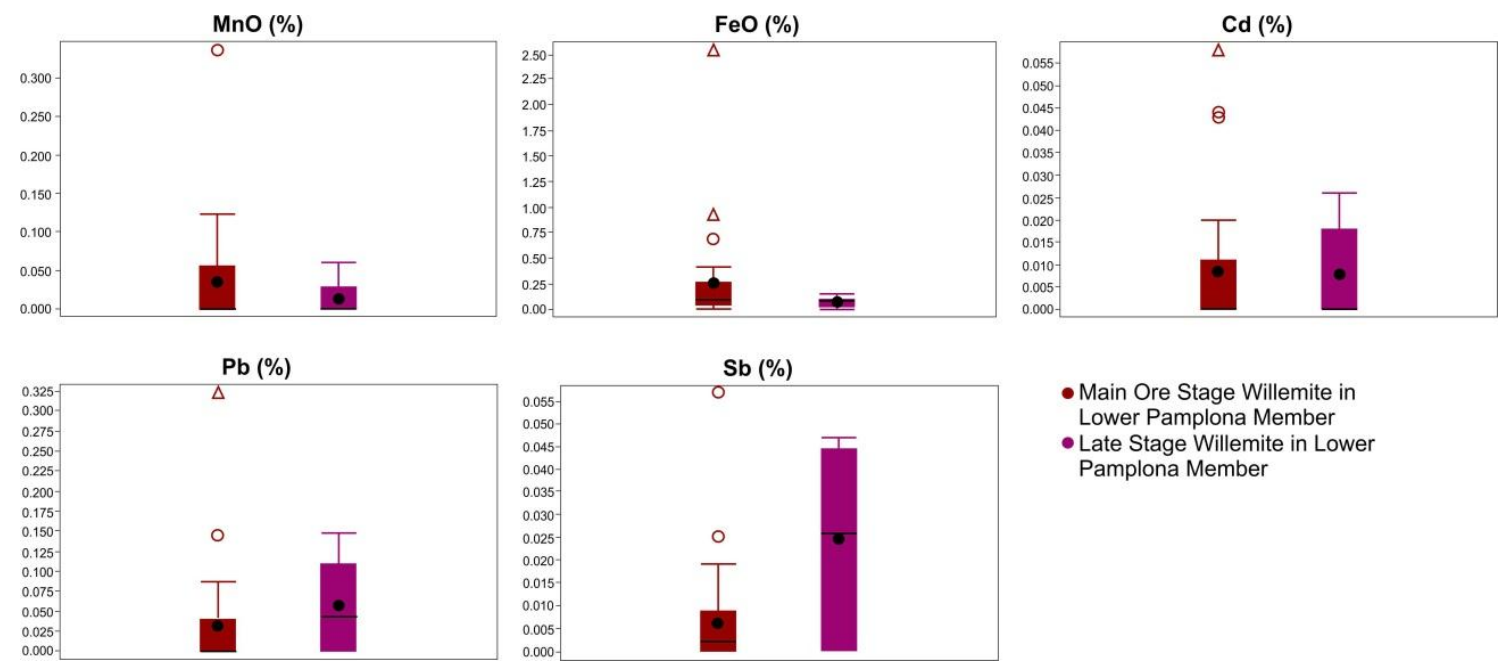

Fig. 2.16. Box and whisker plots showing the variation in composition of different generations of willemite.

\subsubsection{Sphalerite}

Sphalerite was analyzed (Table 2.5; Fig. 2.17) from the pyrite-bearing alteration in the Upper Morro do Pinheiro Member (samples $603.23 \mathrm{~m}$ and $615.40 \mathrm{~m}$ ) and from the galenabearing alteration in the Lower Pamplona Member (sample $411.13 \mathrm{~m}$ ). Sphalerite in the pyrite- 
bearing alteration shows higher content of $\mathrm{Cu}(0.169 \%$ average $)$ and higher content of $\mathrm{Fe}$ $(0.968 \%)$ than the ones from the galena-bearing alteration $(0.091 \%$ and $0.185 \%$ averages, respectively). However, the content of $\mathrm{Cd}$ is higher in sphalerite the galena-bearing alteration in the Lower Pamplona Member $(0.466 \%$ against $0.151 \%$ averages). The average $\mathrm{Pb}$ content from the galena-bearing alteration in the Lower Pamplona Member are slightly higher $(0.097 \%$ against $0.080 \%$ averages) than the pyrite-bearing alteration.

Compared to sphalerite data reported by Monteiro et al. (2006), sphalerite from the pyrite-bearing alteration in the Upper Morro do Pinheiro shows trace elements composition similar to sphalerite from the Ambrósia deposit, whereas the occurrence from the galenabearing alteration in the Lower Pamplona Member is more alike sphalerite from the Fagundes deposit (Fig. 2.18).
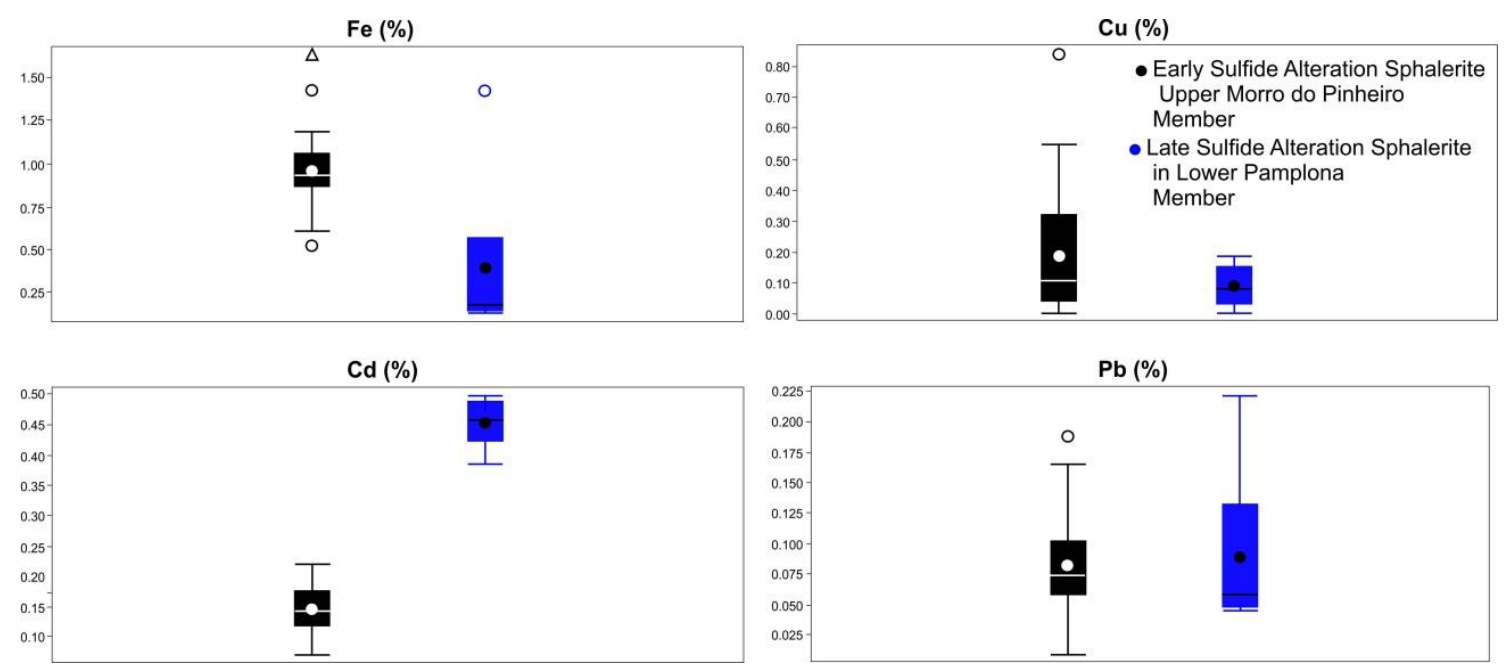

Fig. 2.17. Box and whisker plots showing the variation of different generations of sphalerite.

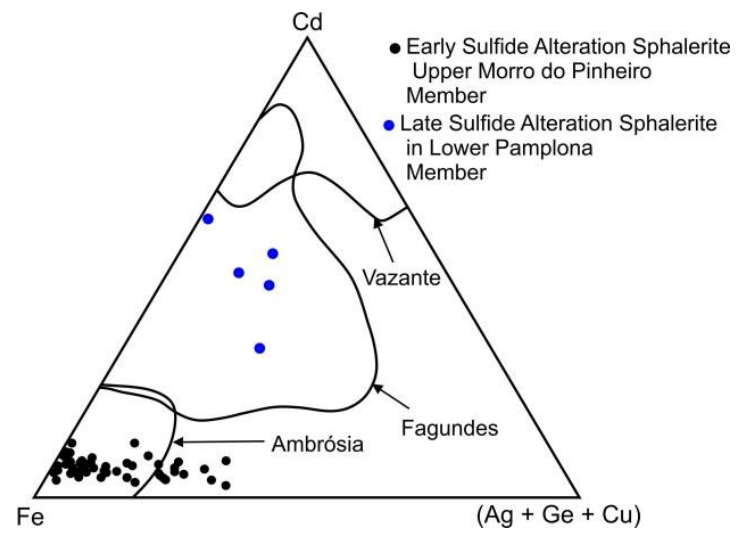

Fig. 2.18. Cd-Fe- $(\mathrm{Ag}+\mathrm{Ge}+\mathrm{Cu})$ atomic ratios for sphalerite from the sulfide alteration in the Upper Morro do Pinheiro and Lower Pamplona members. Atomic ratio fields are from sphalerite from the Ambrósia, Fagundes and Vazante deposits, according to Monteiro et al., (2006).

\subsubsection{Pyrite}

Pyrite compositional data from the pyrite-bearing alteration (Table 2.5; Fig. 2.19) were acquired from samples of the Upper Morro do Pinheiro (samples $603.23 \mathrm{~m}$ and $615.40 \mathrm{~m}$ ) and Lower Pamplona members (sample $273.81 \mathrm{~m}$ ). Higher average contents of $\mathrm{Cd}(0.017 \%)$ are found in pyrite from the Lower Pamplona Member, whereas pyrite from the occurrence at the Upper Morro do Pinheiro Member shows higher average values of $\mathrm{Cu}$ (up to $0.041 \%$ ), $\mathrm{Pb}$ (up to 
$0.163 \%$ ) and $\mathrm{Zn}$ (up to $0.013 \%$ ). Outlier values of $\mathrm{Pb}$ and $\mathrm{Zn}$ can reflect submicroscopic inclusions of galena and sphalerite, respectively. Ag was detected in both pyrite (up to $0.025 \%$ ).
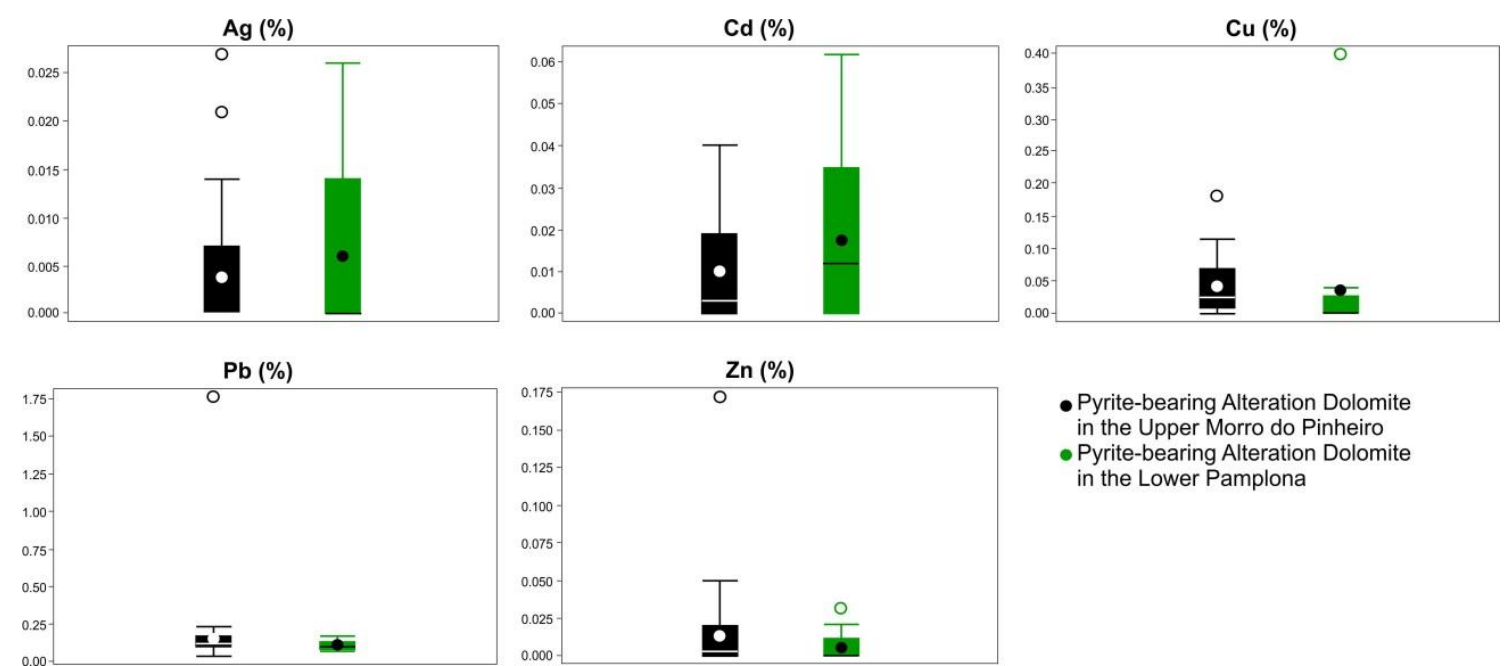

- Pyrite-bearing Alteration Dolomite

in the Upper Morro do Pinheiro

- Pyrite-bearing Alteration Dolomite

in the Lower Pamplona

Fig. 2.19. Box and whisker plots showing the variation of different generations of pyrite.

\subsection{FLUID INCLUSIONS}

\subsubsection{Sample descriptions}

Following up the detailed petrographic examination of the different hydrothermal assemblages, quartz and dolomite grains were selected for fluid inclusion study in five drill core samples, although nine thick sections were examined. Each of these grains is undeformed and is interpreted to be in equilibrium with the mineral assemblage that represents its hydrothermal event or phase. Isolated individual inclusions as well as intra-granular clusters of inclusions were interpreted as primary, whereas those aligned to microfractures or in trails that cross grain boundaries were considered to be secondary.

The selected samples include: (1) One sample from the early stage alteration (sample $305.40 \mathrm{~m}$, Lower Pamplona Member), in which the alteration comprises blocky dolomite substitution bands and nodules; (2) One sample selected from the pre-ore stage alteration (sample $363.73 \mathrm{~m}$, Lower Pamplona Member), in which alteration is represented by large nodules and cavities filled with dog-tooth dolomite on the borders overgrowth by quartz in the central portions; (3) One sample selected from the late ore stage alteration (sample $180.24 \mathrm{~m}$, Lower Pamplona Member), in which quartz analyzed represents the fourth phase (latest) of mineral precipitation of this alteration, as described above; (4 and 5) Two samples of the pyritebearing alteration were selected (samples 267.19 m, Lower Pamplona Member and $603.32 \mathrm{~m}$, Upper Morro do Pinheiro Member), in which this alteration is expressed by white dolomite, quartz and pyrite (hematite is also present in the sample $267.19 \mathrm{~m}$ ). Fluid inclusions in willemite and red dolomite from the main ore stage as well as sphalerite and dolomite from the sulfide alterations could not be analyzed due to the reduced size of the fluid inclusions and low transparency of the host crystals in double polished sections prepared for fluid inclusion studies. 


\subsubsection{Types of Fluid Inclusions}

Six types of fluid inclusions were recognized based on the hydrothermal stage they represent, on their petrographic characteristics and on the microthermometric data (Table 2.6). Type 1 comprises primary biphasic $(\mathrm{L}+\mathrm{V})$ inclusions at room temperature, with degree of filling of 0.95 , and its interpreted composition is $\mathrm{H}_{2} \mathrm{O}-\mathrm{NaCl}-\mathrm{CaCl}_{2}$, based on first ice melting temperatures. These inclusions are rare, colorless, 4 to $5 \mu \mathrm{m}$ in size and were identified in dolomite from the early stage assemblage in the Lower Pamplona Member (Fig. 2.20-A). When observed, the first melting temperature (interpreted as eutectic temperatures; $\mathrm{T}_{\mathrm{e}}$ ) varied from $54.9^{\circ}$ to $-53.1^{\circ} \mathrm{C}$. Final ice melting temperatures $\left(\mathrm{T}_{\mathrm{m}(\mathrm{ice})}\right)$ range from $-12.3^{\circ}$ to $-9.9^{\circ} \mathrm{C}$, and the salinity is between 13.8 and 16.2 wt. $\% \mathrm{NaCl}$ equiv. (Fig. 2.21), calculated according to Bodnar (1993). Total homogenization occurs in the liquid phase and the homogenization temperatures $\left(\mathrm{T}_{\mathrm{h}}\right.$ ) are between $75^{\circ}$ to $97^{\circ} \mathrm{C}$ (Fig. 2.22).
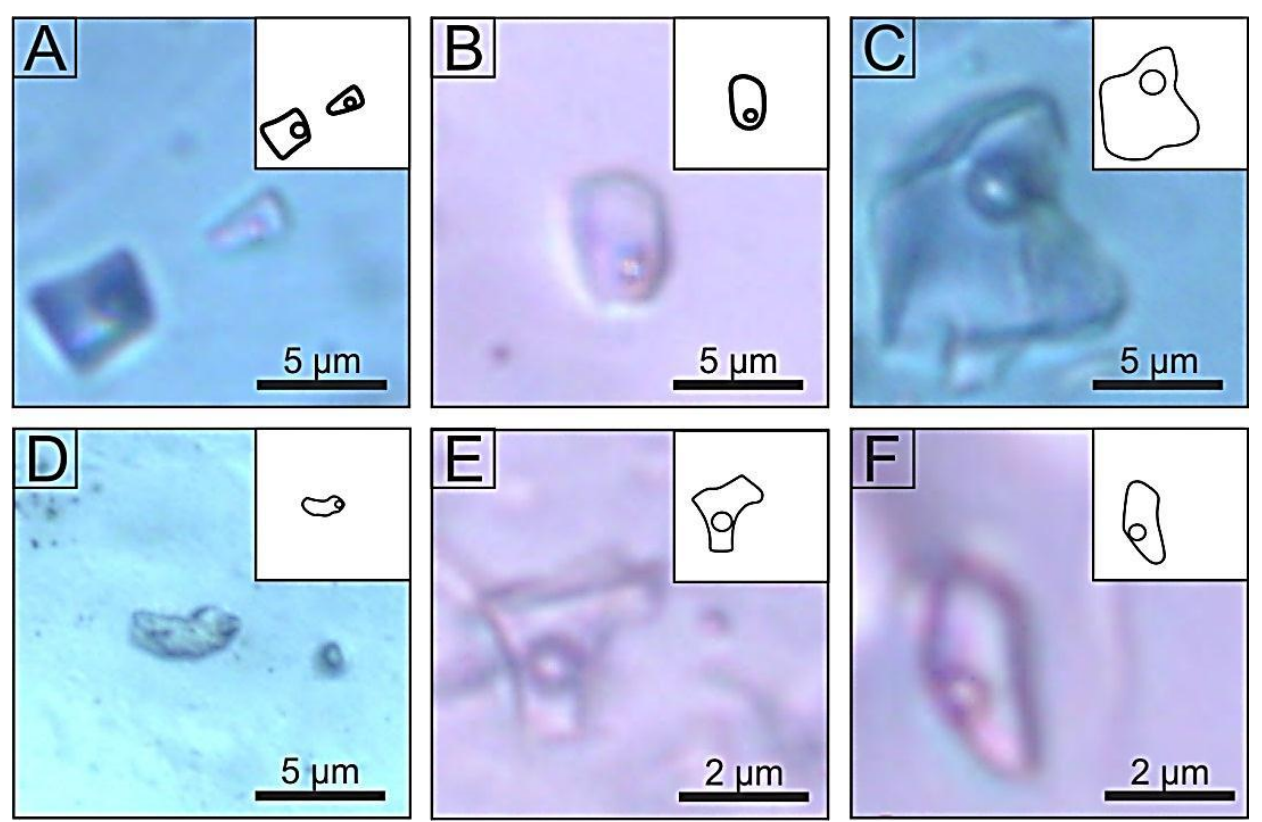

Fig. 2.20. Photomicrographs showing the different fluid inclusions types identified in the VZVG003 drill core. A: Type 1 - two-phase $\mathrm{H}_{2} \mathrm{O}-\mathrm{NaCl}-\mathrm{CaCl}_{2}$ fluid inclusions hosted in dolomite from the early stage alteration in the Lower Pamplona Member (305.40 m). B: Type 2 - two-phase $\mathrm{H}_{2} \mathrm{O}-\mathrm{NaCl}-\mathrm{CaCl}_{2}$ fluid inclusion hosted in dolomite from the pre-ore stage alteration in the Lower Pamplona Member $(363.73 \mathrm{~m})$. C: Type 3 - two-phase $\mathrm{H}_{2} \mathrm{O}-\mathrm{NaCl}-\mathrm{MgCl}_{2}$ fluid inclusion hosted in quartz from the pre-ore stage alteration in the Lower Pamplona Member (363.73 m). D: Type 4 two-phase fluid inclusion of unknown composition hosted in quartz from fourth (late) phase of the ore stage alteration in the Lower Pamplona Member (180.24 m). E: Type 5 - two-phase $\mathrm{H}_{2} \mathrm{O}-\mathrm{NaCl}$ fluid inclusion hosted in quartz from the late phase of the pyrite-bearing alteration in the Lower Pamplona Member $(267.19 \mathrm{~m})$. F: Type 6 - two-phase $\mathrm{H}_{2} \mathrm{O}-\mathrm{NaCl}$ fluid inclusion hosted in quartz from the late phase of the pyrite-bearing alteration in the Upper Morro do Pinheiro Member (603.32 m).

Type 2 comprises primary biphasic $(\mathrm{L}+\mathrm{V})$ inclusions at room temperature, with degree of filling from 0.90 to 0.95 (mode $=0.95$ ), colorless to slightly dark, 1.5 to $4 \mu \mathrm{m}$ in size (Fig. 2.20-B) and are interpreted to be composed of $\mathrm{H}_{2} \mathrm{O}-\mathrm{NaCl}-\mathrm{CaCl}_{2}$. They are hosted in dolomite and quartz from the pre-ore stage assemblage in the Lower Pamplona Member (sample 363.73 $\mathrm{m})$. They show eutectic temperatures $\left(\mathrm{T}_{\mathrm{e}}\right)$ from $-54.4^{\circ}$ to $-52.1^{\circ} \mathrm{C}$, final ice melting temperatures $\left(\mathrm{T}_{\mathrm{m} \text { (ice) }}\right)$ from $-17.1^{\circ}$ to $-14.3^{\circ} \mathrm{C}$, that indicate salinities from 18.0 to $20.3 \mathrm{wt} \%$ $\mathrm{NaCl}$ equiv. Total homogenization $\left(\mathrm{T}_{\mathrm{h}}\right)$ occurs in the liquid phase between $98^{\circ}$ to $125^{\circ} \mathrm{C}$.

Type 3 are primary biphasic $(\mathrm{L}+\mathrm{V})$ inclusions at room temperature, with degree of filling from 0.90 to 0.95 (mode $=0.95$ ), colorless to dark, 1.5 to $16 \mu \mathrm{m}$ in size (Fig. 2.20-C) and are interpreted to be composed of $\mathrm{H}_{2} \mathrm{O}-\mathrm{NaCl}-\mathrm{MgCl}_{2}$. These inclusions were identified in quartz 
and dolomite from the pre-ore stage in the Lower Pamplona Member (sample $363.73 \mathrm{~m}$ ). They exhibit eutectic temperatures $\left(\mathrm{T}_{\mathrm{e}}\right)$ from $-39.7^{\circ}$ to $-35.5^{\circ} \mathrm{C}$, final ice melting temperatures $\left(\mathrm{T}_{\mathrm{m} \text { (ice) }}\right)$ from $-9.8^{\circ}$ to $-4.0^{\circ} \mathrm{C}$, leading to salinities from 6.4 to $13.7 \mathrm{wt} \% \mathrm{NaCl}$ equiv. Total homogenization $\left(\mathrm{T}_{\mathrm{h}}\right)$ occurs in the liquid phase between $105^{\circ}$ to $148^{\circ} \mathrm{C}$.

Table 2.6. Fluid inclusion data for the different fluid inclusion types identified in the various alteration styles in the VZVG003 drill core samples.

\begin{tabular}{|c|c|c|c|c|}
\hline Inclusion type & Hydrothermal alteration & Petrographic characteristics & Microthermometric data & Model system \\
\hline $\begin{array}{l}\text { Type } 1(\mathrm{~L}+\mathrm{V}) \\
(n=5)\end{array}$ & $\begin{array}{l}\text { Early Stage; } \\
\text { hosted in dolomite }\end{array}$ & $\begin{array}{l}\text { Rare, colorless, } 4 \text { to } 5 \mu \mathrm{m} \text {, } \\
\mathrm{F}=0.95 \text {, primary and with } \\
\text { random distribution }\end{array}$ & $\begin{array}{l}\mathrm{T}_{\mathrm{e}}=54.9^{\circ} \text { to }-53.1^{\circ} \mathrm{C} \\
\text { (mode }=-53.1^{\circ} \mathrm{C} \text { ); } \\
\mathrm{T}_{\text {m(ic) }}=-12.3^{\circ} \text { to }-9.9^{\circ} \mathrm{C} \text {; } \\
(13.8 \text { to } 16.2 \text { wt. } \% \mathrm{NaCl} \text { equiv.) } \\
\mathrm{T}_{\mathrm{h}(\mathrm{L})}=75^{\circ} \text { to } 97^{\circ} \mathrm{C}\end{array}$ & $\mathrm{H}_{2} \mathrm{O}-\mathrm{NaCl}-\mathrm{CaCl}_{2}$ \\
\hline $\begin{array}{l}\text { Type } 2(\mathrm{~L}+\mathrm{V}) \\
(n=30)\end{array}$ & $\begin{array}{l}\text { Pre-Ore Stage; } \\
\text { hosted in quartz and } \\
\text { dolomite }\end{array}$ & $\begin{array}{l}\text { Random, colorless to slightly } \\
\text { dark, } 1.5 \text { to } 4.0 \mu \mathrm{m}, \mathrm{F}=0.90 \text { to } \\
0.95(\text { mode }=0.95) \text { and primary }\end{array}$ & $\begin{array}{l}\mathrm{T}_{\mathrm{e}}=-54.4^{\circ} \text { to }-52.1^{\circ} \mathrm{C} \\
\mathrm{T}_{\mathrm{m} \text { (ice) }}=-17.1^{\circ} \text { to }-14.3^{\circ} \mathrm{C} \\
(18.0 \text { to } 20.3 \text { wt. } \% \mathrm{NaCl} \text { equiv. } \\
\text { mode }=20.1 \text { wt. } \% \mathrm{NaCl} \text { equiv.) } \\
\mathrm{T}_{\mathrm{h}(\mathrm{L})}=98^{\circ} \text { to } 125^{\circ} \mathrm{C}\end{array}$ & $\mathrm{H}_{2} \mathrm{O}-\mathrm{NaCl}-\mathrm{CaCl}_{2}$ \\
\hline $\begin{array}{l}\text { Type } 3(\mathrm{~L}+\mathrm{V}) \\
(n=26)\end{array}$ & $\begin{array}{l}\text { Pre-Ore Stage; } \\
\text { hosted in quartz and } \\
\text { dolomite }\end{array}$ & $\begin{array}{l}\text { Random, colorless to dark, } \\
1.5 \text { to } 16 \mu \mathrm{m}, \mathrm{F}=0.90 \text { to } 0.95 \\
\text { (mode }=0.95 \text { ) and primary }\end{array}$ & $\begin{array}{l}\mathrm{T}_{\mathrm{c}}=-39.7 \text { to }-35.5 \\
\mathrm{~T}_{\mathrm{m} \text { (ice) }}=-9.8^{\circ} \text { to }-4.0^{\circ} \mathrm{C} \\
(6.4 \text { to } 13.7 \text { wt. } \% \mathrm{NaCl} \text { equiv. }) \text {; } \\
\mathrm{T}_{\mathrm{h}(\mathrm{L})}=105^{\circ} \text { to } 148^{\circ} \mathrm{C}\end{array}$ & $\mathrm{H}_{2} \mathrm{O}-\mathrm{NaCl}-\mathrm{MgCl}_{2}$ \\
\hline $\begin{array}{l}\text { Type } 4(\mathrm{~L}+\mathrm{V}) \\
(n=33)\end{array}$ & $\begin{array}{l}\text { Ore Stage Late phases } \\
\text { and Py-bearing Alteration; } \\
\text { hosted in quartz }\end{array}$ & $\begin{array}{l}\text { Random, colorless, dark, } 1.5 \text { to } \\
4.0 \mu \mathrm{m}, \mathrm{F}=0.90 \text { to } 0.95 \\
\text { (mode }=0.95 \text { ) and primary }\end{array}$ & $\begin{array}{l}\mathrm{T}_{\mathrm{e}}=* ; \\
\mathrm{T}_{\mathrm{m} \text { (ice) }}=-6.5^{\circ} \text { to }-3.6^{\circ} \mathrm{C} \\
(5.8 \text { to } 9.9 \text { wt. } \% \mathrm{NaCl} \text { equiv. }) \text {; } \\
\mathrm{T}_{\mathrm{h}(\mathrm{L})}=90^{\circ} \text { to } 178^{\circ} \mathrm{C}\end{array}$ & $*$ \\
\hline $\begin{array}{l}\text { Type } 5(\mathrm{~L}+\mathrm{V}) \\
(n=35)\end{array}$ & $\begin{array}{l}\text { Ore Stage Late phases } \\
\text { and Py-bearing Alteration; } \\
\text { hosted in quartz }\end{array}$ & $\begin{array}{l}\text { Random, colorless to dark, } \\
1.0 \text { to } 4.0 \mu \mathrm{m}, \mathrm{F}=0.80 \text { to } 0.97 \\
\text { (mode }=0.95 \text { ) and primary }\end{array}$ & $\begin{array}{l}\mathrm{T}_{\mathrm{e}}=-22.6 \text { to }-21.1 ; \\
\mathrm{T}_{\mathrm{m} \text { (ice) }}=-14.7 \text { to }-8.4^{\circ} \mathrm{C} \\
(12.2 \text { to } 18.4 \text { wt. } \% \mathrm{NaCl} \text { equiv. }) \text {; } \\
\mathrm{T}_{\mathrm{h}(\mathrm{L})}=92^{\circ} \text { to } 196^{\circ} \mathrm{C}\end{array}$ & $\mathrm{H}_{2} \mathrm{O}-\mathrm{NaCl}$ \\
\hline $\begin{array}{l}\text { Type } 6(\mathrm{~L}+\mathrm{V}) \\
(n=23)\end{array}$ & $\begin{array}{l}\text { Py-bearing Alteration; } \\
\text { hosted in quartz }\end{array}$ & $\begin{array}{l}\text { Random, colorless, } \\
2.0 \text { to } 4.0 \mu \mathrm{m}, \mathrm{F}=0.95 \\
\text { and primary }\end{array}$ & $\begin{array}{l}\mathrm{T}_{\mathrm{e}}=-55.2 \text { to }-52.4^{\circ} \mathrm{C} \\
\mathrm{T}_{\mathrm{m} \text { (ice) }}=-10.6 \text { to }-4.6^{\circ} \mathrm{C} \\
(7.3 \text { to } 14.6 \text { wt. } \% \mathrm{NaCl} \text { equiv. }) \\
\mathrm{T}_{\mathrm{h}(\mathrm{L})}=124^{\circ} \text { to } 152^{\circ} \mathrm{C}\end{array}$ & $\mathrm{H}_{2} \mathrm{O}-\mathrm{NaCl}-\mathrm{CaCl}_{2}$ \\
\hline
\end{tabular}

Abbreviations: $\mathrm{F}=$ degree of filling, $\mathrm{Py}=$ pyrite $\mathrm{T}_{\mathrm{e}}=$ eutectic temperature, $\mathrm{T}_{\mathrm{m}(\mathrm{icc})}=$ temperature of final ice melting, $\mathrm{T}_{\mathrm{h}(\mathrm{L})}=$ total homogenization temperature (to the liquid)

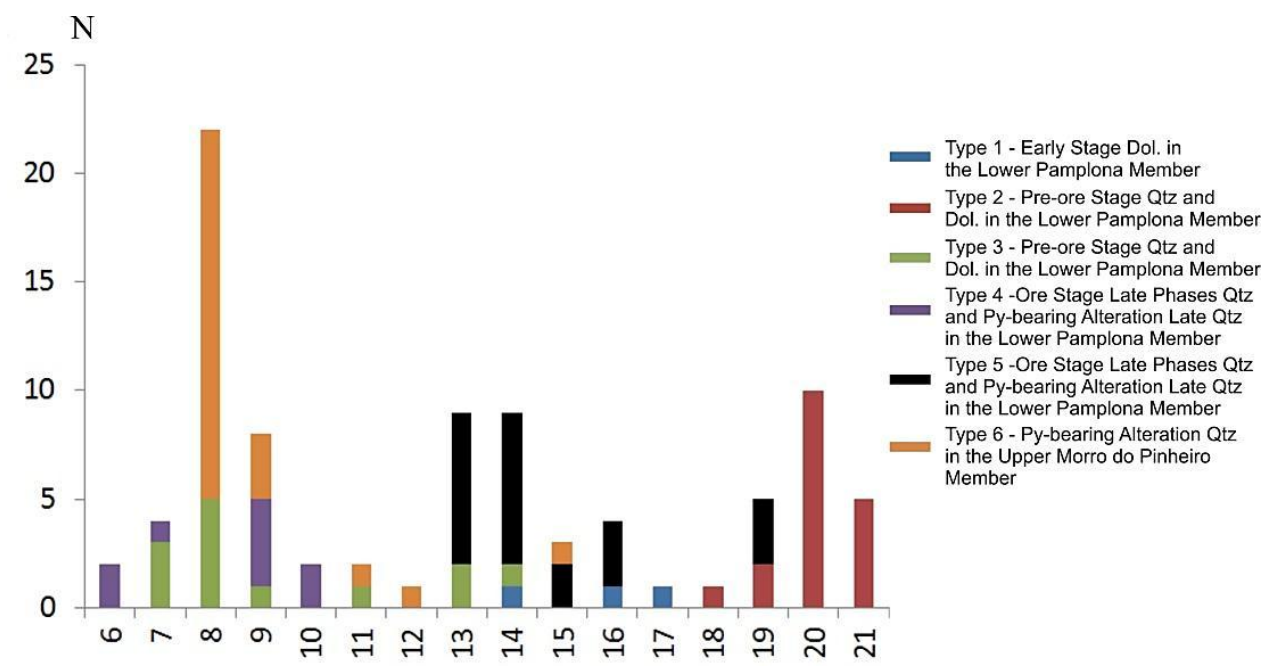

Wt. $\% \mathrm{NaCl}$ equiv.

Fig. 2.21. Salinity (wt. \% NaCL equiv.) for the different fluid inclusions identified at the various alterations styles in the VZVG003 drill core samples. 


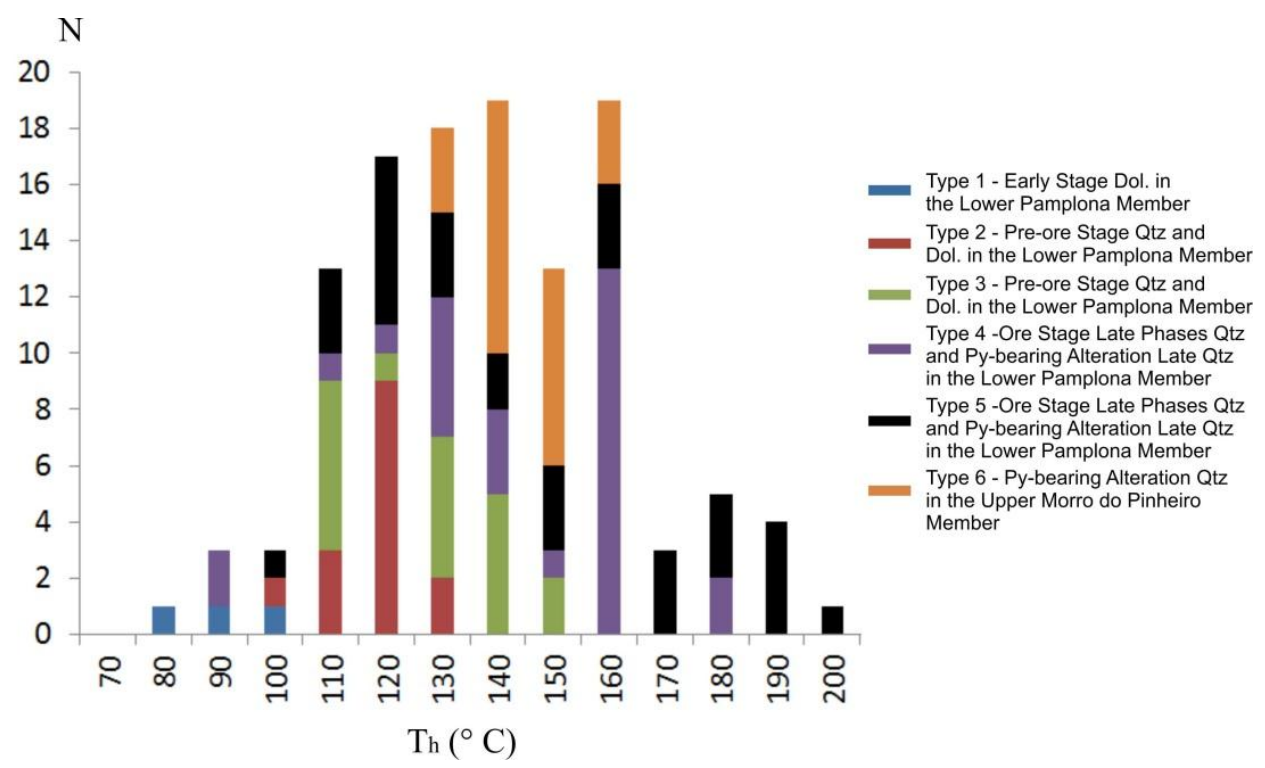

Fig. 2.22. Homogenization temperatures $\left(T_{h}\right)$ for the different fluid inclusions identified at the various alteration styles in VZVG003 drill core samples.

Type 4 was identified in quartz from the latest phase of mineral precipitation of the ore stage (sample $180.24 \mathrm{~m}$ ) and in quartz from the pyrite-bearing alteration (sample $267.19 \mathrm{~m}$ ) which is late to dolomite and pyrite (Fig. 2.14-B), both in the Lower Pamplona Member. They are primary biphasic $(\mathrm{L}+\mathrm{V})$ inclusions at room temperature, with degree of filling from 0.90 to 0.95 (mode $=0.95$ ), colorless to dark, 1.5 to $4 \mu \mathrm{m}$ in size (Fig. 2.20-D). Their composition is unknown due to the non-visualization of first ice melting, leading to absence of eutectic temperature data for this type. Final ice melting temperatures $\left(\mathrm{T}_{\mathrm{m} \text { (ice) }}\right)$ were between $-6.5^{\circ} \mathrm{C}$ to $-3.6^{\circ}$, that corresponds to salinities from 5.8 to 9.9 wt. $\% \mathrm{NaCl}$ equiv. Total homogenization $\left(\mathrm{T}_{\mathrm{h}}\right.$ ) occurs in the liquid phase between $90^{\circ}$ to $178^{\circ} \mathrm{C}$.

Type 5 comprises primary biphasic $(\mathrm{L}+\mathrm{V})$ inclusions at room temperature, with degree of filling from 0.80 to 0.97 (mode $=0.95$ ), colorless to dark, 1 to $4 \mu \mathrm{m}$ in size (Fig. 2.20-E). They are hosted in quartz from late phase of the ore stage (sample $180.24 \mathrm{~m}$ ) and late quartz from the pyrite-bearing alteration (sample $267.19 \mathrm{~m}$ ), both in the Lower Pamplona Member, and interpreted to be composed of $\mathrm{H}_{2} \mathrm{O}-\mathrm{NaCl}$. They show eutectic temperatures $\left(\mathrm{T}_{\mathrm{e}}\right)$ from $-22.6^{\circ}$ to $21.1^{\circ} \mathrm{C}$. Final ice melting temperatures $\left(\mathrm{T}_{\mathrm{m} \text { (ice) }}\right)$ range from $-14.7^{\circ}$ to $-8.4^{\circ} \mathrm{C}$, indicating salinities from 12.2 to $18.4 \mathrm{wt} \% \mathrm{NaCl}$ equiv. Total homogenization $\left(\mathrm{T}_{\mathrm{h}}\right)$ occurs in the liquid phase between $92^{\circ}$ to $196^{\circ} \mathrm{C}$.

Type 6 comprehends colorless primary biphasic $(\mathrm{L}+\mathrm{V})$ inclusions at room temperature, with degree of filling of $0.95,2$ to $4 \mu \mathrm{m}$ in size (Fig. 2.20-F) and are hosted in quartz from the pyrite-bearing alteration in the Upper Morro do Pinheiro Member (sample $603.32 \mathrm{~m}$ ), interpreted to be composed of $\mathrm{H}_{2} \mathrm{O}-\mathrm{NaCl}-\mathrm{CaCl}_{2}$. They show eutectic temperatures $\left(\mathrm{T}_{\mathrm{e}}\right)$ from $55.2^{\circ}$ to $-52.4^{\circ} \mathrm{C}$, final ice melting temperatures $\left(\mathrm{T}_{\mathrm{m} \text { (ice) }}\right)$ range from $-10.6^{\circ}$ to $-4.6^{\circ} \mathrm{C}$, indicating salinities from 7.3 to $14.6 \mathrm{wt}$. $\% \mathrm{NaCl}$ equiv. Total homogenization $\left(\mathrm{T}_{\mathrm{h}}\right)$ occurs in the liquid phase between $124^{\circ}$ to $152^{\circ} \mathrm{C}$. 


\subsection{STABLE ISOTOPES}

Carbon and oxygen stable isotope analyses were performed in 68 dolomite grains and their isotope compositions as well as the calculated isotope compositions of the fluids in equilibrium with them are summarized in figures 2.23-A and B, respectively. The temperatures used for the isotope compositions of the fluids were based on the fluid inclusion data and were calculated according to Horita (2014). Figure 2.24 shows the isotope values of the samples throughout the drill hole.

The measured $\delta^{13} \mathrm{C} \mathrm{PDB}$ and $\delta^{18} \mathrm{O}$ SMOW values are, respectively, (i) $+0.79 \%$ to $+2.88 \%$ and $+23.79 \%$ to $+29.74 \%$ for dolomite from the least altered dolostones $(n=19)$, (ii) $+1.48 \%$ to $+2.50 \%$ and $+25.30 \%$ to $+26.65 \%$ for early stage dolomite from substitution bands and nodules $(n=3)$, (iii) $+1.49 \%$ to $+2.26 \%$ and $+26.02 \%$ to $+27.41 \%$ or pre-ore stage dogtooth and blocky dolomite $(n=4)$, (iv) $-1.10 \%$ and $+29.85 \%$ for saddle dolomite from the first phase of the ore stage $(n=1)$, (v) $+0.26 \%$ o to $+1.23 \%$ and $+19.39 \%$ o to $+22.82 \%$ o for red dolomite from main ore stage ( $n=10)$, (vi) $+1.23 \%$ to $+1.61 \%$ and $+26.22 \%$ to $+27.55 \%$ for white dolomite from incipient third phase of the ore stage $(n=2)$, (vii) $-1.62 \%$ to $+1.41 \%$ and $+16.46 \%$ to $+21.51 \%$ for white dolomite from pervasive third phase of the ore stage $(n=12)$, (viii) $+2.09 \%$ to $+2.92 \%$ and $+27.22 \%$ to $+30.02 \%$ for white dolomite from incipient pyritebearing alteration $(n=6)$ and (ix) $+0.37 \%$ o to $+2.27 \%$ ond $+19.12 \%$ o to $+25.73 \%$ o for white dolomite from pervasive pyrite-bearing alteration.

The calculated $\delta^{13} \mathrm{C}$ PDB and $\delta^{18} \mathrm{O}$ SMOW values for the fluids in equilibrium with the different dolomite occurrences are, respectively, (i) $-3.62 \%$ to $-2.60 \%$ and $+4.60 \%$ o to +5.95 at $90^{\circ} \mathrm{C}$ for early stage dolomite from substitution bands and nodules, (ii) $-1.81 \%$ to $-1.04 \%$ and $+8.82 \%$ to $+10.21 \%$ at $120^{\circ} \mathrm{C}$ for pre-ore stage dog-tooth and blocky dolomite, (iii) $-1.80 \%$ and $+17.65 \%$ at $180^{\circ} \mathrm{C}$ for saddle dolomite from the first phase of the ore stage, (iv) $-0.44 \%$ to $+0.53 \%$ and $+7.19 \%$ o to $+10.62 \%$ at $180^{\circ} \mathrm{C}$ for red dolomite from the main ore stage, (v) $+0.53 \%$ to $+0.91 \%$ and $+14.02 \%$ to $+15.35 \%$ at $180^{\circ} \mathrm{C}$ for white dolomite from incipient third phase of the ore stage, (vi) $-2.32 \%$ to $+0.71 \%$ and $+4.26 \%$ to $+9.31 \%$ at $180^{\circ} \mathrm{C}$ for white dolomite from pervasive third phase of the ore stage, (vii) $-0.41 \%$ to $+0.42 \%$ and $+11.52 \%$ to $+14.32 \%$ at $180^{\circ} \mathrm{C}$ for white dolomite from incipient pyrite-bearing alteration, and (viii) $2.13 \%$ to $-0.23 \%$ and $+3.42 \%$ to $+10.03 \%$ at $180^{\circ} \mathrm{C}$ for white dolomite from pervasive pyritebearing alteration.

The isotopic signatures from the early stage alteration dolomite (substitution bands) as well as from the pre-ore stage dolomite are within the field of concentration of values of $\delta^{13} \mathrm{C}$ and $\delta^{18} \mathrm{O}$ from the least altered dolostones, showing very similar values between them. White dolomite from incipient third phase of the ore stage and from incipient pyrite-bearing alteration also show the same pattern, with signature similar to the least altered dolostones (Fig. 2.23-A). This suggests that the isotope composition of these dolomites was buffered by the composition of the least altered dolostones.

Dolomite from the pervasive ore stage and pervasive pyrite-bearing alterations, however, shows lighter carbon and oxygen isotope compositions. This trend is enhanced in dolomite co-genetic to willemite (Fig. 2.23-A).

The isotope composition of the fluids in equilibrium with dolomite from the early stage exhibit the lightest isotopic composition, followed by dolomite from the pre-ore stage. This suggests presence of organic carbon in the early stages of fluid flow in the basin. Isotope compositions of the fluids in equilibrium with white dolomite from incipient third phase of the ore stage and from incipient pyrite-bearing alteration show isotopic signatures closer to the least altered dolostones, suggesting that the isotopic composition of the fluid was buffered by the 
composition of the host dolostones (possibly by the dissolution and re-precipitation). Nonetheless, the evolution of the ore stage and the pyrite-bearing alteration show a lighting trend of carbon and oxygen isotope composition (purple dashed arrow in Figure 2.23-B). Similar trend was documented (Baia, 2013; Monteiro et al., 2007; Oliveira, 2013; Percy, 2014; Rubo and Monteiro, 2010; see page 40, section 2.4) and is interpreted to be associated with interaction of hydrothermal fluids with carbonaceous phyllite.
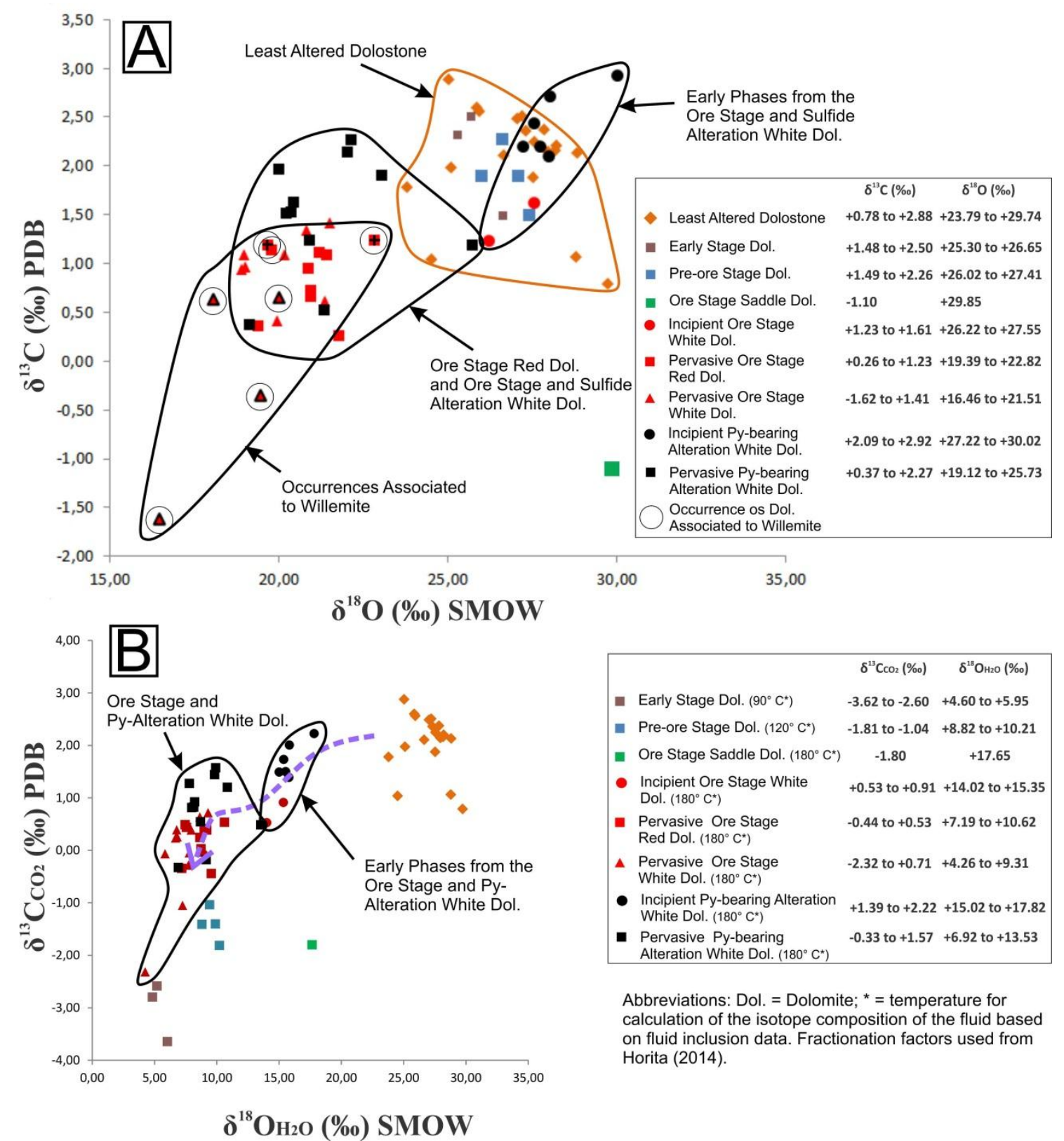

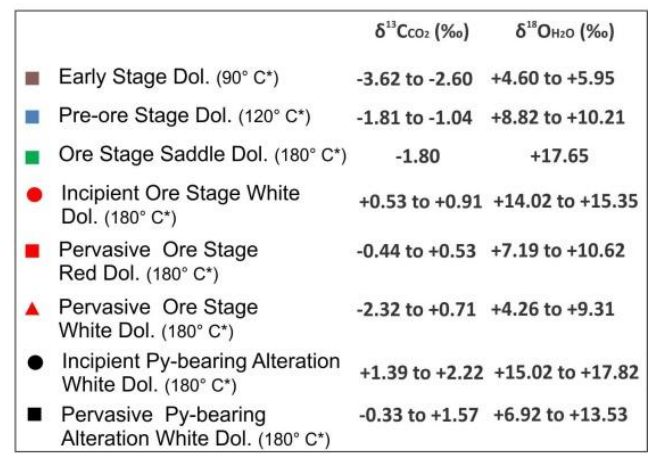

Abbreviations: Dol. $=$ Dolomite $;{ }^{*}=$ temperature for calculation of the isotope composition of the fluid based on fluid inclusion data. Fractionation factors used from Horita (2014)

Fig. 2.23. Binary diagrams of the oxygen and carbon isotope composition of (A) different occurrences of dolomite and the (B) calculated isotope compositions of the fluid in equilibrium with them. Purple dashed arrow shows the trend of isotope composition accompanying the evolution of the ore stage and pyrite-bearing alterations. 


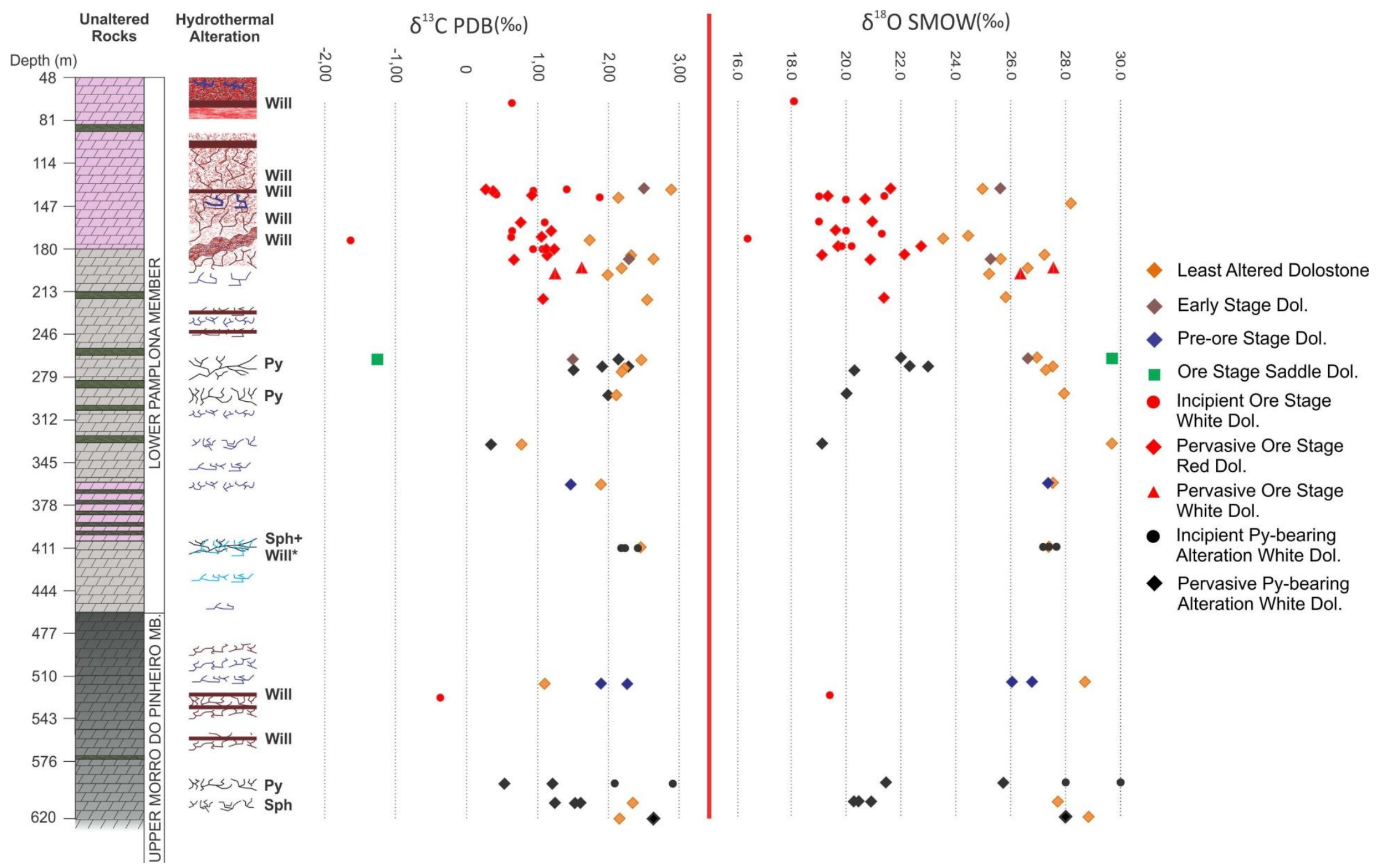




\subsection{LITHOGEOCHEMISTRY}

For whole rock geochemistry 28 samples were selected based on the hydrothermal phase and stage they represent: samples from the pre-ore stage alteration in the Upper Morro do Pinheiro and Lower Pamplona members $(n=3)$; samples from the early phase of the ore stage alteration in the Lower Pamplona Member $(n=2)$, characterized by red stained dolostones (e.g.: Fig. 2.10-A); samples from the main ore stage marked by massive precipitation of red dolomite and hematite in the Upper Morro do Pinheiro $(n=2)$; and in the Lower Pamplona Member $(n=$ 7; e.g.: Fig. 2.10-B, C); samples from the third phase of the ore stage represented by precipitation of white dolomite that substitutes red dolomite in the Upper Morro do Pinheiro and Lower Pamplona members $(n=3$; e.g.: figs. 2.10-E,F); samples from the pyrite-bearing alteration in the Upper Morro do Pinheiro and Lower Pamplona members $(n=5)$; and unaltered dolostones of the Upper Morro do Pinheiro and Lower Pamplona members $(n=5)$ for qualitative comparison. The whole rock geochemistry results are displayed in tukey box plot diagrams on the figs. 2.25 to 2.30 .

The pre-ore stage alteration shows a considerable higher concentrations of $\mathrm{SiO}_{2}$. Early phase of the ore stage alteration is characterized by higher concentrations of $\mathrm{Al}_{2} \mathrm{O}_{3}, \mathrm{P}_{2} \mathrm{O}_{5}$, Dy, $\mathrm{Er}, \mathrm{Eu}, \mathrm{Gd}, \mathrm{Nd}, \mathrm{Pr}, \mathrm{Sm}, \mathrm{Tb}$, and Ni. The main ore stage shows higher concentrations of $\mathrm{SiO}_{2}$, $\mathrm{Fe}_{2} \mathrm{O}_{3}, \mathrm{P}_{2} \mathrm{O}_{5}, \mathrm{Mn}, \mathrm{As}, \mathrm{Ge}, \mathrm{Hg}, \mathrm{In}, \mathrm{U}, \mathrm{V}, \mathrm{Ag}, \mathrm{Cd}, \mathrm{Cu}, \mathrm{Mo}, \mathrm{Ni}, \mathrm{Pb}, \mathrm{Sb}, \mathrm{Se}$ and $\mathrm{Zn}$, and very low sulfur content (below detection limit). In the Upper Morro do Pinheiro Member, the main ore stage exhibit also higher concentrations of $\mathrm{Co}$, and higher concentrations of $\mathrm{Cu}$ and $\mathrm{Zn}$ ( $\mathrm{Zn}$ up to $39.8 \%$ against $33.9 \%$ in the overlying member). The Lower Pamplona Member mineralized zone has also anomalous $\mathrm{Sb}$ and yielded higher concentrations of $\mathrm{As}, \mathrm{Mn}, \mathrm{Pb}$, and $\mathrm{V}$. The third phase of the ore stage alteration, which is characterized by white dolomite precipitation, shows higher concentrations of $\mathrm{Fe}_{2} \mathrm{O}_{3}, \mathrm{Mn}, \mathrm{Ba}, \mathrm{Cr}, \mathrm{Cd}, \mathrm{Ni}, \mathrm{Pb}$, and As. The pyrite-bearing alteration is marked by higher concentrations of $\mathrm{Na}_{2} \mathrm{O}, \mathrm{Fe}_{2} \mathrm{O}_{3}, \mathrm{SiO}_{2}, \mathrm{Ba}, \mathrm{Cu}, \mathrm{Ni}$, As, and $\mathrm{S}$.

There are similar higher concentrations of $\mathrm{TiO}_{2}, \mathrm{Ba}, \mathrm{Ni}$, and As among the late phase of the ore stage alteration and the sulfide alteration. Depletion in major elements that constitutes the host dolostones in this alterations phases represents dissolution and substitution processes (e.g.: $\mathrm{Ca}$ and $\mathrm{Mg}$ ). 

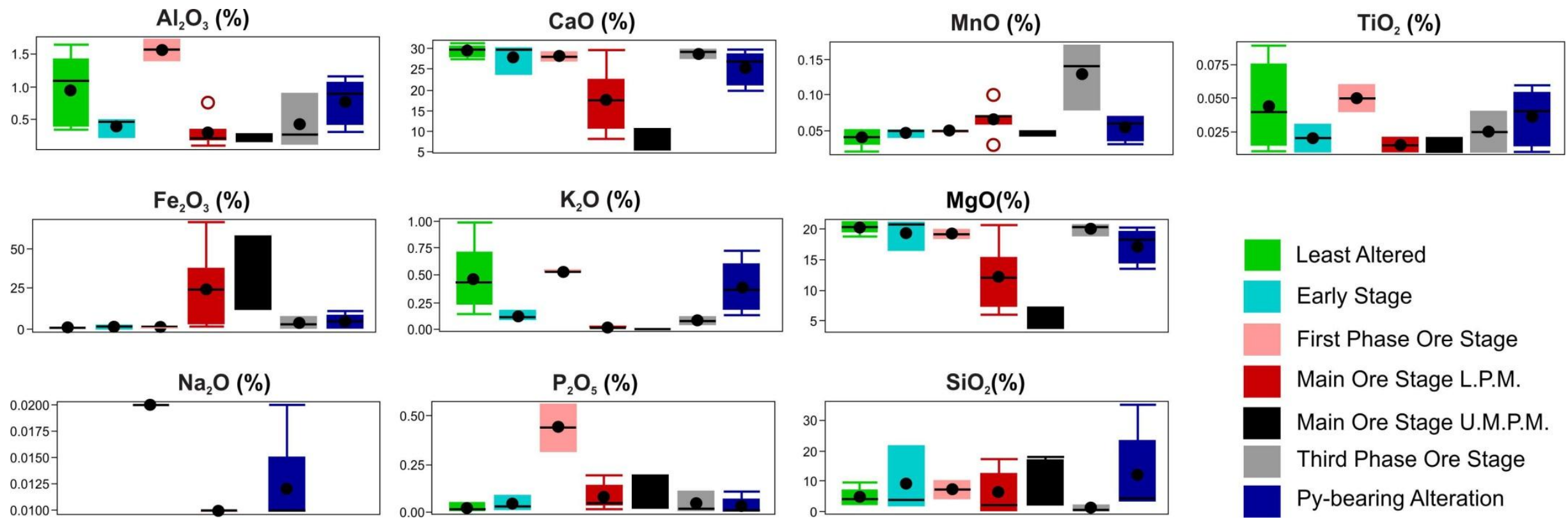

Fig. 2.25. Box and whisker plots showing the variation in composition of the major elements for the various alteration styles recognized in the VZVG003 drill hole. L.P.M. = Lower Pamplona Member; U.M.P.M. = Upper Morro do Pinheiro Member. 

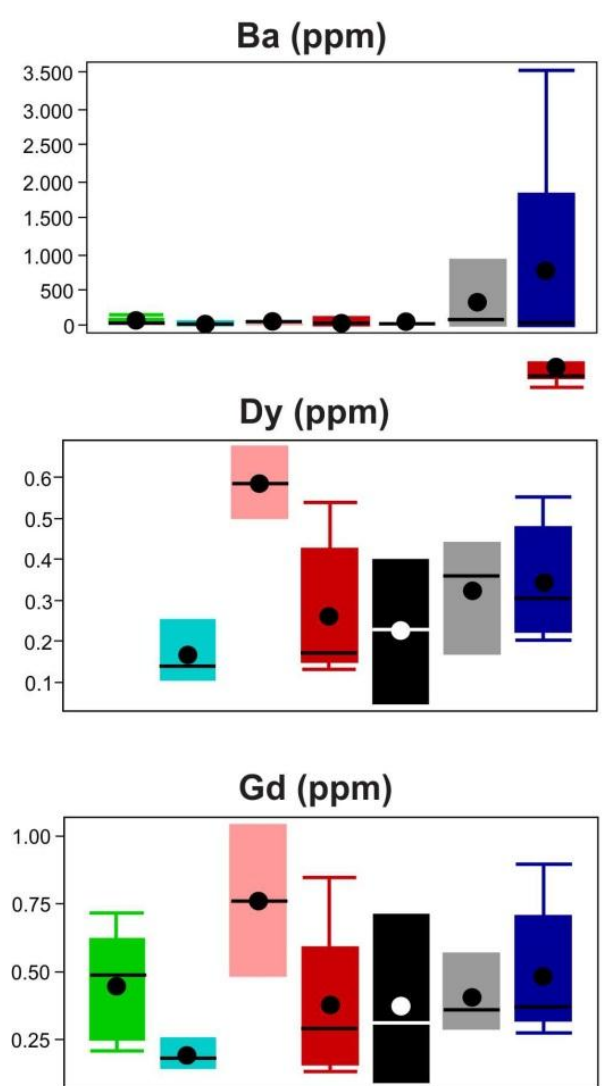

Least Altered Early Stage
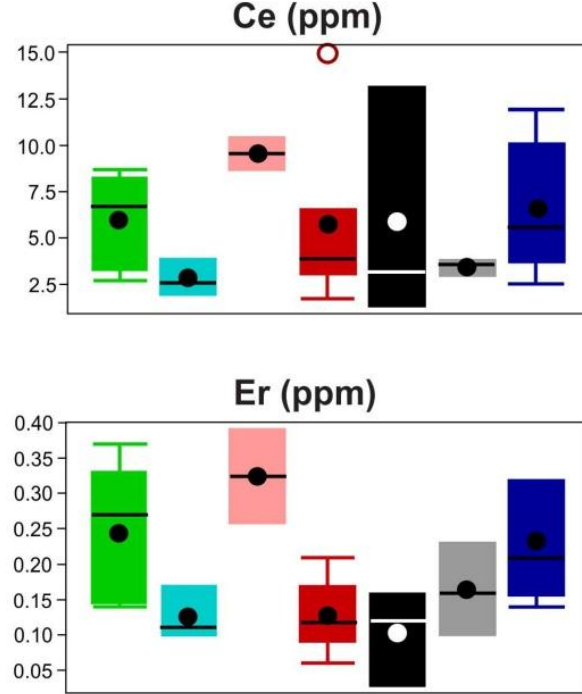

Ge (ppm)

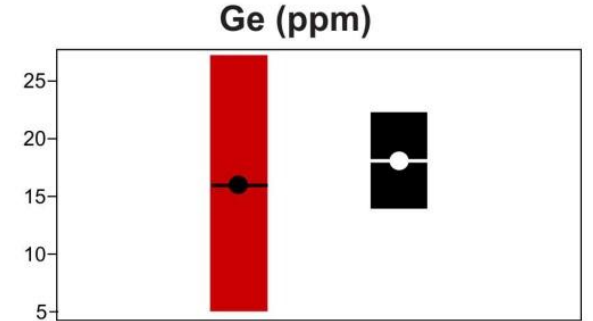

First Phase Ore Stage Main Ore Stage L.P.M.
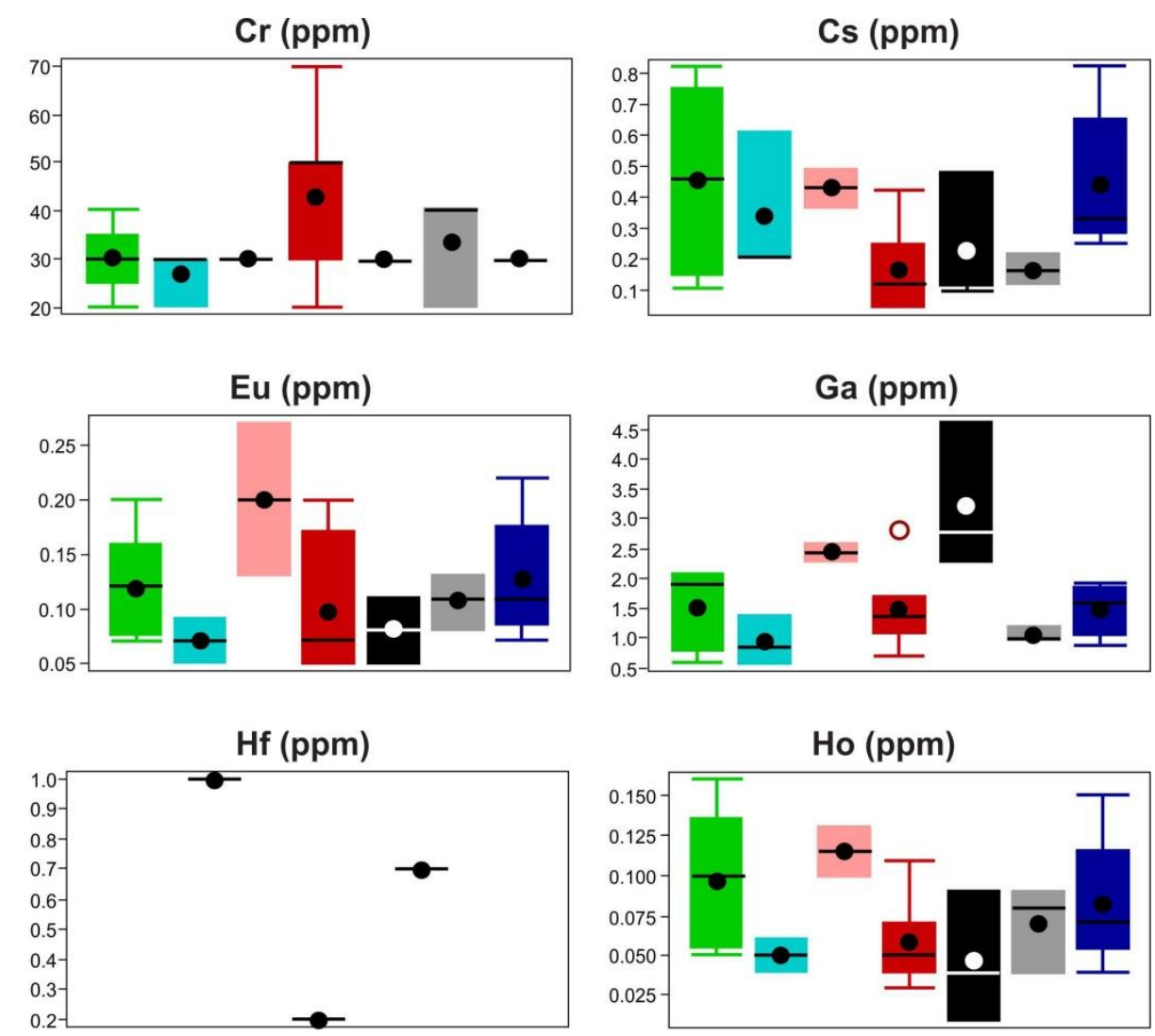

Main Ore Stage U.M.P.M

Third Phase Ore Stage

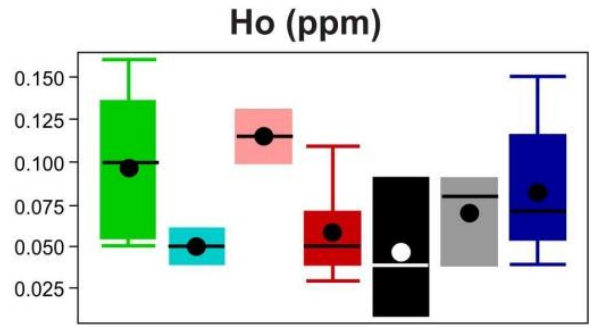

Py-bearing Alteration

Fig. 2.26. Box and whisker plots showing the variation in composition of the elements from $\mathrm{Ba}-\mathrm{Ho}$ for the various alteration styles recognized in the VZVG003 drill hole. L.P.M. = Lower Pamplona Member; U.M.P.M. = Upper Morro do Pinheiro Member. 

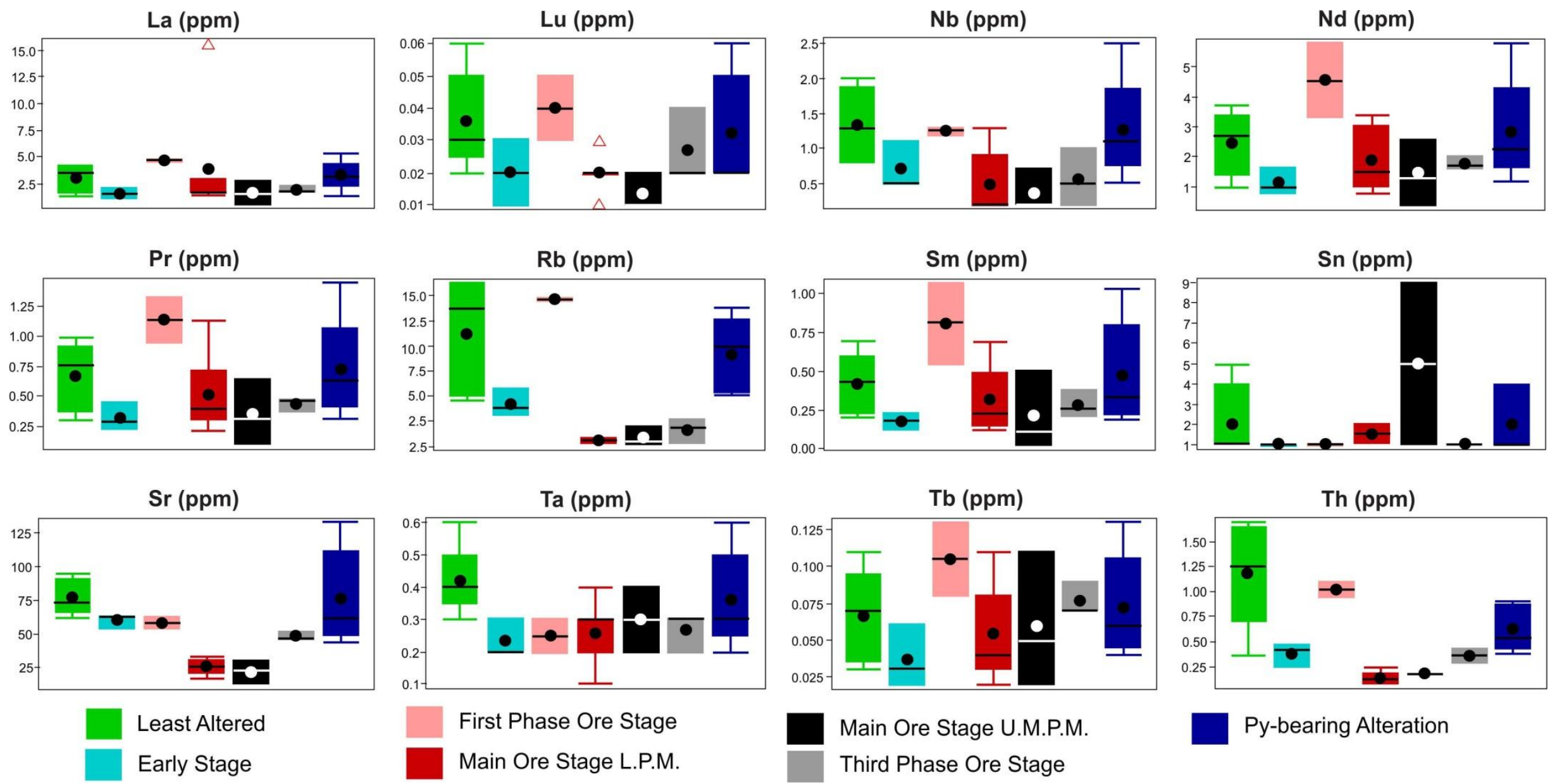

Fig. 2.27. Box and whisker plots showing the variation in composition of the elements from La - Th for the various alteration styles recognized in the VZVG003 drill hole. L.P.M. = Lower Pamplona Member; U.M.P.M. = Upper Morro do Pinheiro Member. 

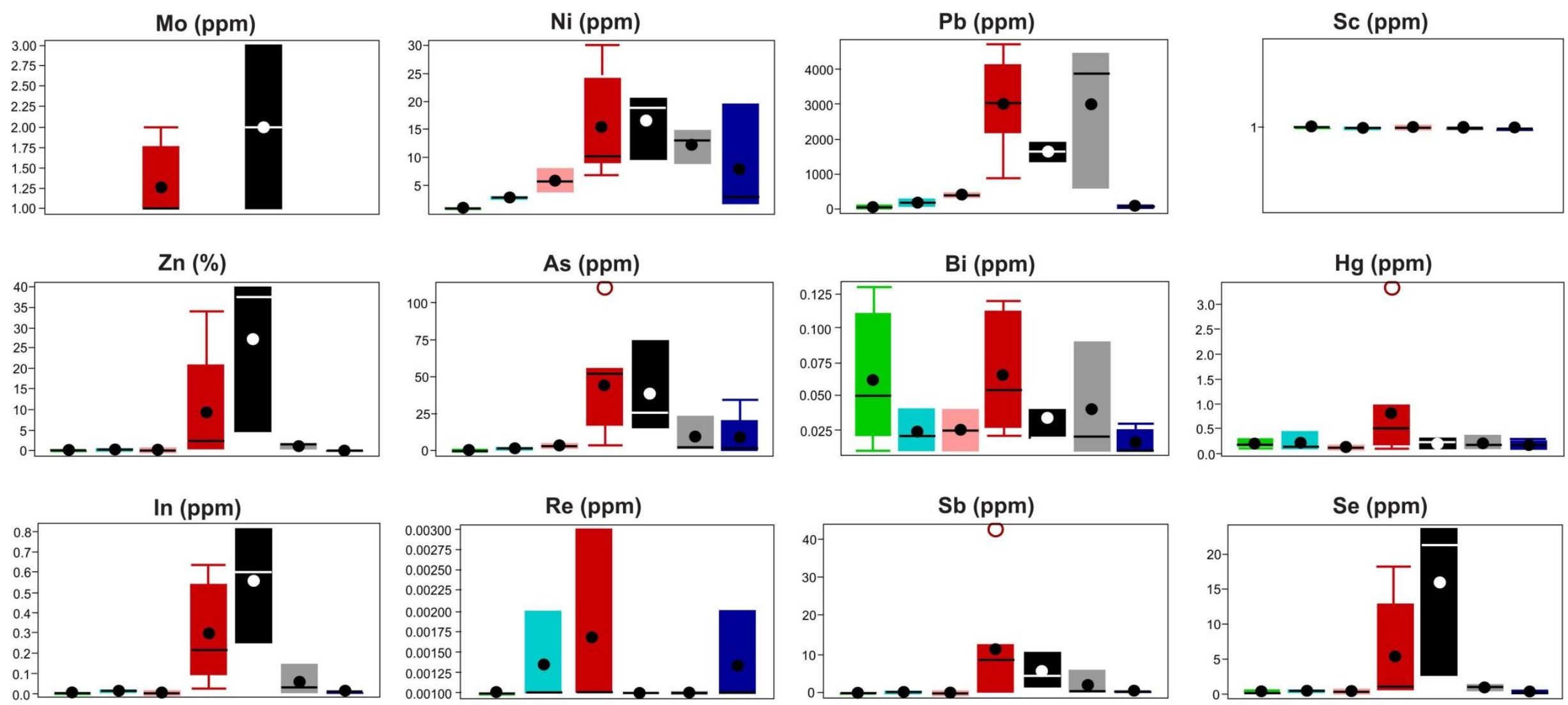

Least Altered

First Phase Ore Stage

Early Stage

Main Ore Stage L.P.M.

Main Ore Stage U.M.P.M

Third Phase Ore Stage

Fig. 2.28. Box and whisker plots showing the variation in composition of the elements from Mo - Se for the various alteration styles recognized in the VZVG003 drill hole. L.P.M. = Lower Pamplona Member; U.M.P.M. = Upper Morro do Pinheiro Member. 

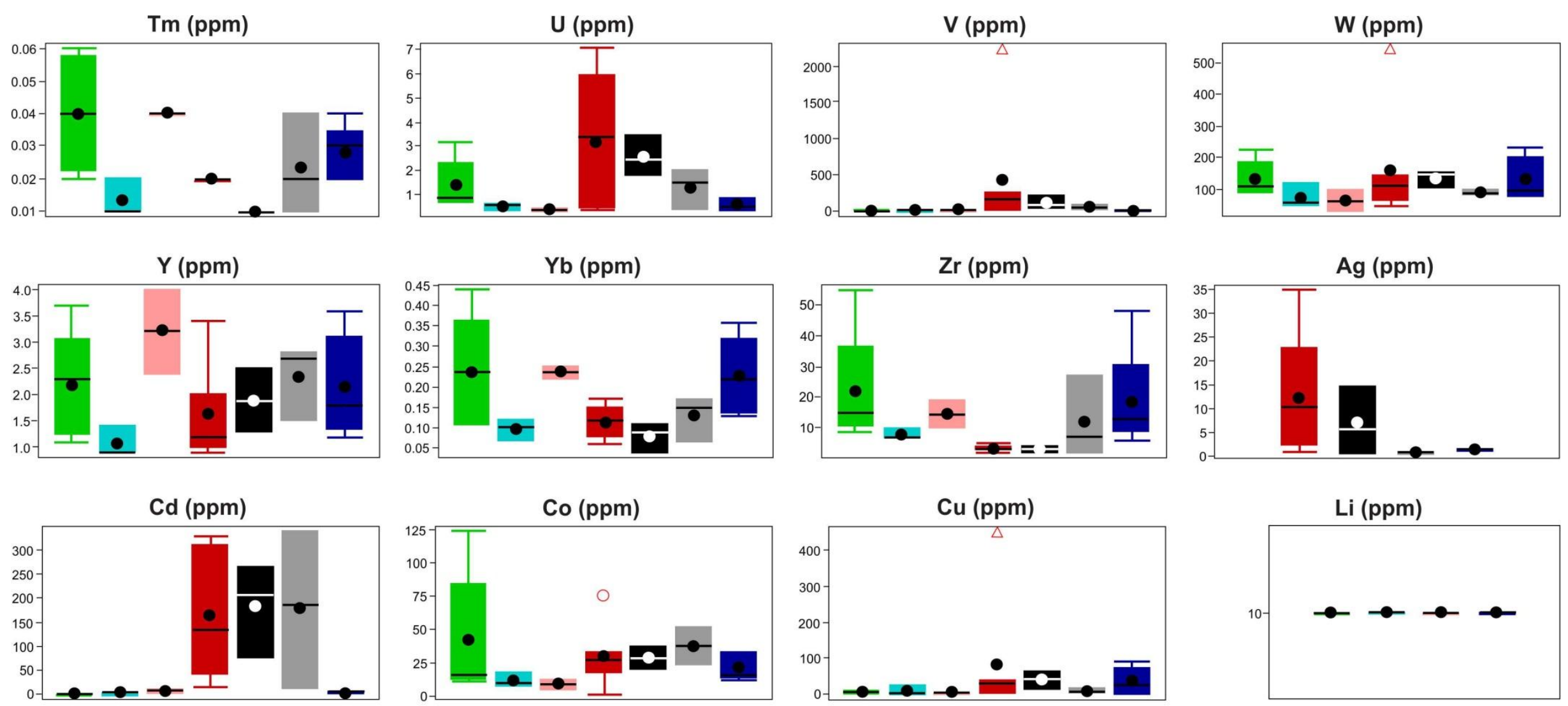

Least Altered

First Phase Ore Stage

Early Stage

Main Ore Stage L.P.M.

Main Ore Stage U.M.P.M.

Py-bearing Alteration

Third Phase Ore Stage

Fig. 2.29. Box and whisker plots showing the variation in composition of the elements from Tm - Li for the various alteration styles recognized in the VZVG003 drill hole. L.P.M. = Lower Pamplona Member; U.M.P.M. = Upper Morro do Pinheiro Member. 

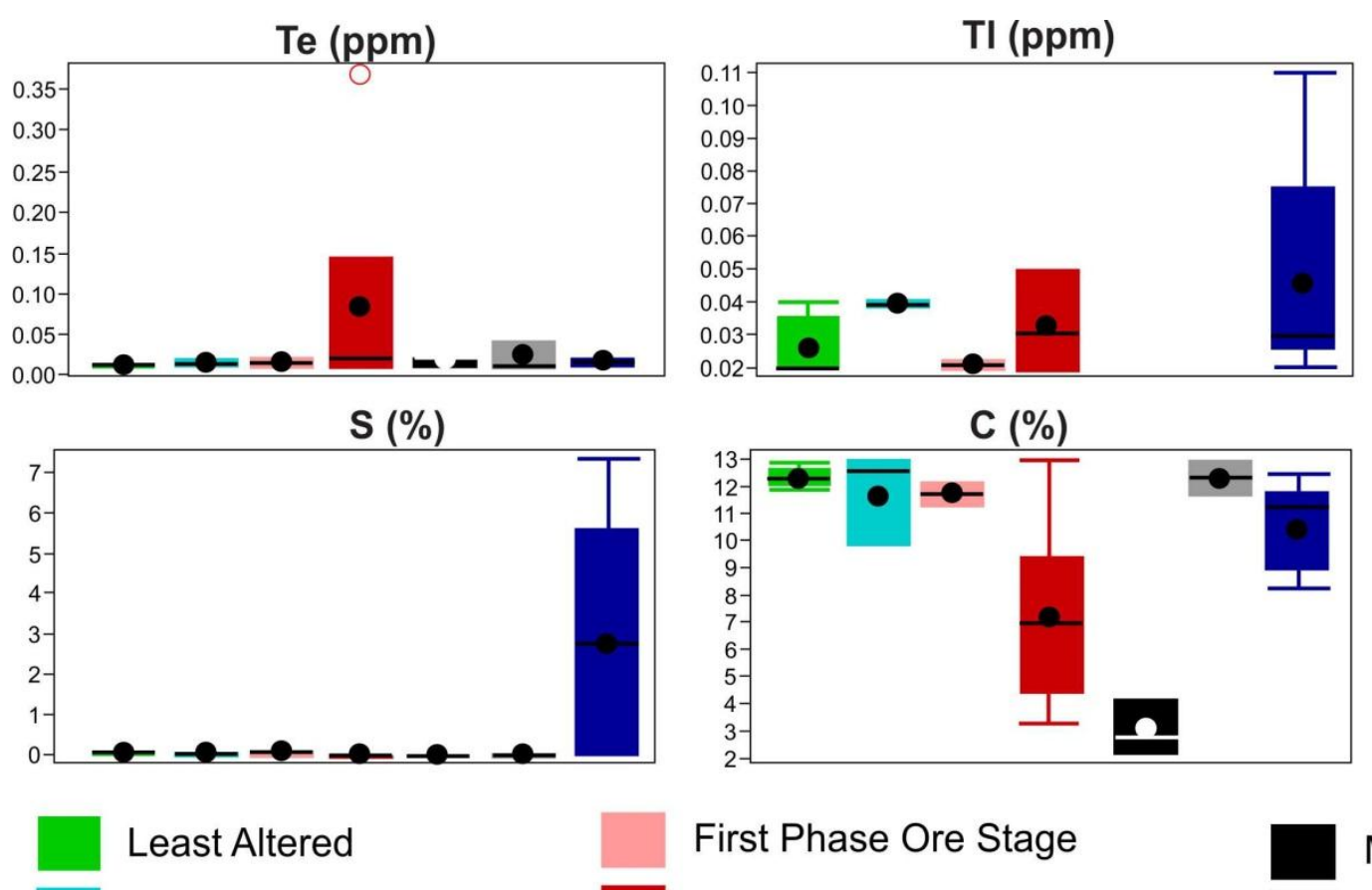

First Phase Ore Stage

Main Ore Stage U.M.P.M. Third Phase Ore Stage

Fig. 2.30. Box and whisker plots showing the variation in composition of the elements Te, Tl, S, and C for the various alteration styles recognized in the VZVG003 drill hole. L.P.M. $=$ Lower Pamplona Member; U.M.P.M. = Upper Morro do Pinheiro Member. 


\subsection{DISCUSSSIONS}

\subsubsection{Evolution of the hydrothermal systems in the Southern Extension of the Vazante Group}

The study of the Southern Extension of the Vazante Group revealed various stages of hydrothermal alteration, which are summarized in figures 2.6 and 2.31. The earliest hydrothermal event documented in the Vazante Group is characterized by the dolomite substitution bands preferentially sub-parallel to the sedimentary laminations. This dolomite is poor in trace elements, showing discrete higher concentrations of $\mathrm{Fe}, \mathrm{Mn}$ and $\mathrm{Cd}$ (Table 2.4; Fig. 2.15). The composition of the fluids associated to this event is $\mathrm{H}_{2} \mathrm{O}-\mathrm{NaCl}-\mathrm{CaCl}_{2}$ with salinities of 13.8 to $16.2 \mathrm{wt}$. $\% \mathrm{NaCl}$ equiv. (Table 2.6; Fig. 2.21), and with homogenization temperatures around $75^{\circ}$ to $97^{\circ} \mathrm{C}$ (Table 2.6; Fig. 2.22). Their calculated $\mathrm{C}$ and $\mathrm{O}$ isotopic compositions are lighter than the subsequent events (Fig. 2.23-B). This hydrothermal alteration is interpreted as resulted from processes of dissolution of carbonate and re-precipitation of dolomite during late diagenesis. These moderate salinity fluids with lighter $\mathrm{C}$ and $\mathrm{O}$ isotopic compositions suggest that the fluids were from meteoric and/or connate water which has interacted with evaporites and organic matter or hydrocarbons, which are reported in the Lower Pamplona Member (Dardenne et al., 1998; Oliveira 2013; Slezak et al., 2014).

The second hydrothermal event (pre-ore stage) is mainly characterized by dog-tooth and blocky dolomite and quartz precipitated in dissolution voids. They have formed by mixing of fluids, as indicated by the presence of $\mathrm{H}_{2} \mathrm{O}-\mathrm{NaCl}-\mathrm{CaCl}_{2}$ fluid inclusions, with salinities hanging from 18.0 to 20.3 wt. \% NaCl equiv. and homogenization temperatures between $98^{\circ}$ to $125^{\circ} \mathrm{C}$, and $\mathrm{H}_{2} \mathrm{O}-\mathrm{NaCl}-\mathrm{MgCl}_{2}$ fluid inclusions, with salinities of 6.4 to 13.7 wt. $\% \mathrm{NaCl}$ equiv., and homogenization temperatures from $105^{\circ}$ to $148^{\circ} \mathrm{C}$ (Table 2.6). Carbon isotopic composition of the fluids in equilibrium in dolomite is between $-1.81 \%$ to $-1.04 \%$, which is heavier than the early stage alteration but in average lighter than the subsequent phases, supporting interaction with light $\mathrm{C}$. This alteration exhibits higher concentrations of $\mathrm{SiO}_{2}$ but is poor in ore-related elements, suggesting that they have not interacted with the mineralizing fluids.

The silicate zinc ore-related hydrothermal alterations comprise three major phases. The first one is characterized by a distal reddish dolostone documented mainly in the Lower Pamplona Member with geochemical signature marked by higher concentrations of $\mathrm{Al}_{2} \mathrm{O}_{3}, \mathrm{P}_{2} \mathrm{O}_{5}$, and some rare earth elements (REE: Ce, Dy, Er, Eu, Ga, Gd, Nd, Pr, Sm, Tb and Y), and discrete higher concentrations of $\mathrm{Ni}, \mathrm{Pb}$, and $\mathrm{As}$ (Appendix 1). Unfortunately fluid inclusions were not identified in these altered rocks. Although this alteration commonly occurs as distal halos in the outer zones around the mineralized cores, their geochemical signature is very distinct. Therefore the genetic link between this style of alteration and the mineralization is not clear.

The second phase of the ore stage alteration is the main ore stage, and comprises the high grade zones in the Upper Morro do Pinheiro (480 to $570 \mathrm{~m}$ ) and Lower Pamplona (48 to $210 \mathrm{~m}$ ) members. It is typically represented by dissolution breccias with massive $\mathrm{Zn}$-bearing red dolomite ( $\sim 1 \%$ in average $\mathrm{Zn}$, and $\sim 0.5 \%$ in average $\mathrm{Fe}$ ), abundant hematite and willemite $(\sim 0.25 \%$ in average $\mathrm{Fe})$, forming massive bodies in the Upper Morro do Pinheiro mineralized interval and more patchy arrays in the Lower Pamplona zone. Hydraulic breccias are observed mainly in the Lower Pamplona Member zone (Fig. 2.6), suggesting that it formed in shallower zones, with lower lithostatic pressure and/or higher hydrothermal fluid pressure. The overall geochemical signature of the main ore stage alteration is characterized by higher concentrations 
of $\mathrm{SiO}_{2}, \mathrm{Fe}_{2} \mathrm{O}_{3}, \mathrm{P}_{2} \mathrm{O}_{5}, \mathrm{Mn}, \mathrm{As}, \mathrm{Ge}, \mathrm{Hg}, \mathrm{In}, \mathrm{U}, \mathrm{V}, \mathrm{Ag}, \mathrm{Cd}, \mathrm{Cu}, \mathrm{Mo}, \mathrm{Ni}, \mathrm{Pb}, \mathrm{Sb}$, Se, and $\mathrm{Zn}$. In the Upper Morro do Pinheiro Member there is also higher concentrations of Co, and higher concentrations of $\mathrm{Cu}$ and $\mathrm{Zn}$ ( $\mathrm{Zn}$ up to 39.8\% against 33.9\% in the overlying member). The Lower Pamplona Member mineralized zone has also anomalous $\mathrm{Sb}$ and yielded higher grades of As, $\mathrm{Mn}, \mathrm{Pb}$, and $\mathrm{V}$.
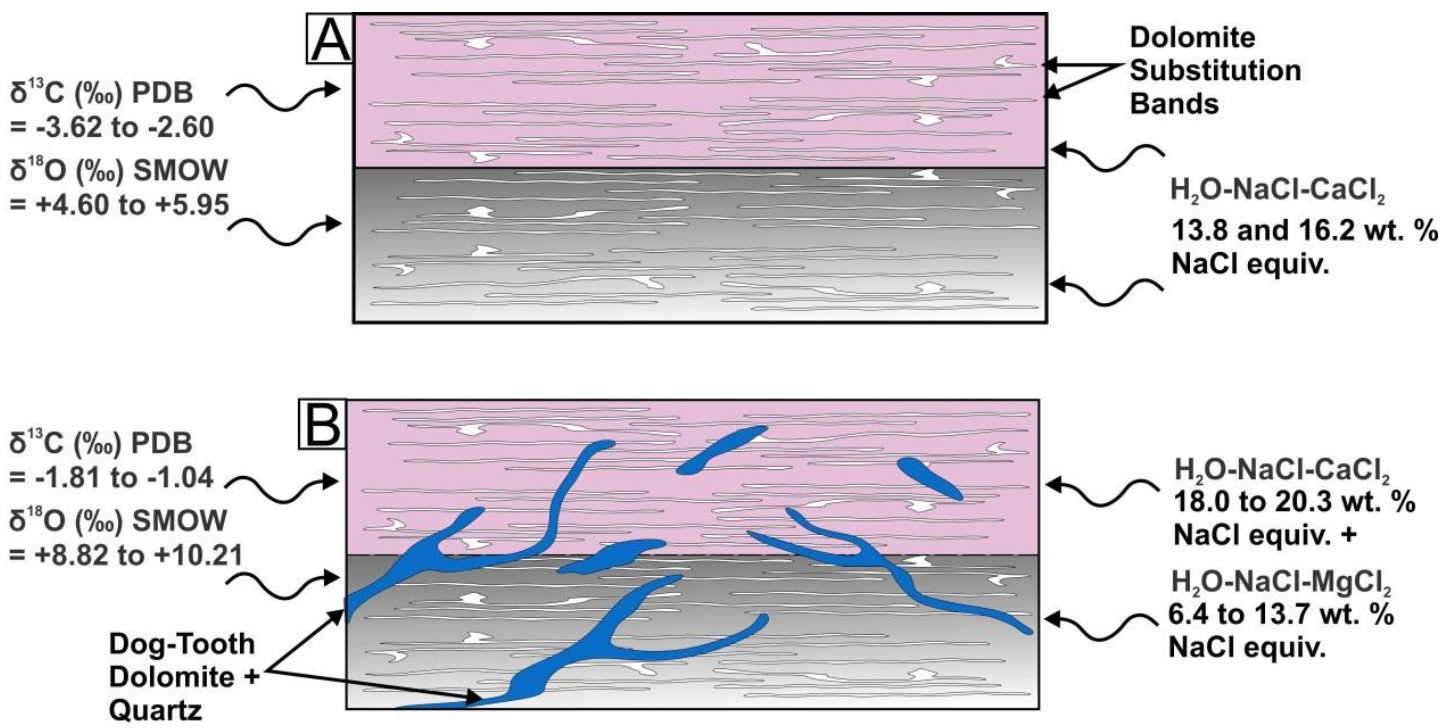

Quartz

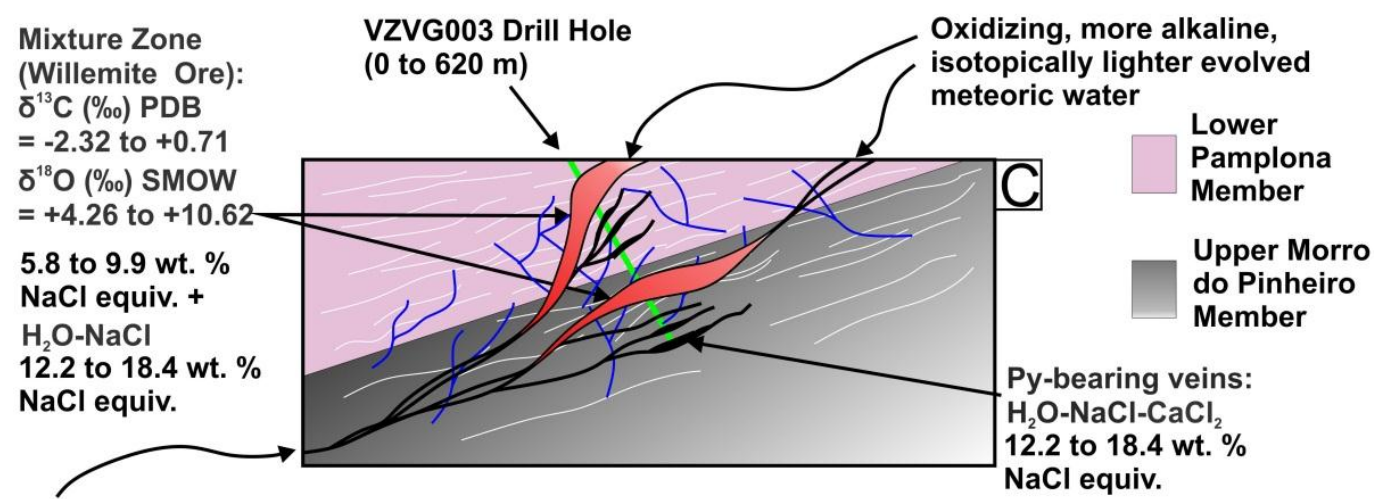

$\mathrm{SiO}_{2}, \mathrm{Fe}_{2} \mathrm{O}_{3}, \mathrm{P}_{2} \mathrm{O}_{5}, \mathrm{Mn}, \mathrm{As}$,

$\mathrm{Ge}, \mathrm{Hg}, \mathrm{In}, \mathrm{U}, \mathrm{V}, \mathrm{W}, \mathrm{Ag}, \mathrm{Cd}$,

$\mathrm{Cu}, \mathrm{Mo}, \mathrm{Ni}, \mathrm{Pb}, \mathrm{Sb}, \mathrm{Se}, \mathrm{Zn}$

Fig. 2.31. Evolution of the hydrothermal system in the Upper Morro do Pinheiro and Lower Pamplona members from the Serra do Poço Verde Formation, in the Southern Extension of the Vazante Group. A: Early stage; B: Pre-ore stage; and C: Ore stage and pyrite-bearing (early sulfide) alteration.

The third ore-related phase with white dolomite and minor hematite and willemite is characterized by higher concentrations of $\mathrm{Mn}, \mathrm{Ba}, \mathrm{Cr}, \mathrm{Cd}, \mathrm{Ni}, \mathrm{Pb}$, and $\mathrm{As}$. The isotopic carbon and oxygen signature of carbonates of second and third phases are similar $(+0.26$ to $+1.23 \% /+19.39$ to $+22.82 \%$ and -1.62 to $+1.41 \% /+16.46$ to $+21.51 \%$, respectively) and lighter carbon isotopic compositions are found in dolomite from the second (main ore) phase coeval with willemite (Fig. 2.23-A). This suggests that at willemite precipitation sites, mixing with lighter $\mathrm{C}$ and $\mathrm{O}$ isotopes fluids was enhanced. The lighter $\mathrm{O}$ isotopic composition might be due to higher involvement of evolved meteoric water whereas lighter $\mathrm{C}$ isotopic compositions suggest that hydrothermal fluids interacted with organic carbon-bearing rocks or that bacteriological processes were involved during this hydrothermal phase. These fluids were sulfur deficient (contents below the detection limits), indicating that this possible interaction 
with organic carbon did not supplied sulfur. No fluid inclusion data was obtained for these phases.

The fourth ore-related phase, which is comprised of quartz (quartz 3, Fig 2.7), and zincian chlorite, revealed two types of fluid inclusions with distinct salinities (5.8 to 9.9 and 12.2 to 18.4 wt. $\% \mathrm{NaCl}$ equiv.) and wide ranges of homogenization temperatures $\left(90^{\circ}\right.$ to $178^{\circ}$ $\mathrm{C}$, and $92^{\circ}$ to $196^{\circ} \mathrm{C}$, respectively). The higher salinity fluid inclusions show higher correlation to higher homogenization temperatures (Fig. 2.32; higher $\mathrm{T}_{\mathrm{h}}$ values from type 5 fluid inclusions are of higher salinities than type 4 fluid inclusions). The higher salinity fluid inclusions showed eutectic temperatures typical of $\mathrm{H}_{2} \mathrm{O}-\mathrm{NaCl}$ system, however first ice melting was not observed in the less saline fluids. These data suggest that mixing of two fluids, one of higher salinity and temperatures $\left(\sim 170^{\circ}\right.$ to $\left.190^{\circ} \mathrm{C}\right)$ and another lower salinity and temperatures $\left(\sim 90^{\circ}\right.$ to $\left.130^{\circ} \mathrm{C}\right)$, were involved in the late ore-stage alteration.

The pyrite-bearing alteration style, with pyrite, sphalerite, white dolomite, quartz and fine hematite, yielded $\mathrm{FeO}$ contents in white dolomite in the Upper Morro do Pinheiro and Lower Pamplona members averaging $1.389 \%$ and $2.474 \%$, respectively (Table 2.4). Variations in the content of $\mathrm{FeO}$ and $\mathrm{Zn}$ in dolomite from the pyrite-bearing alteration in the Upper Morro do Pinheiro and Lower Pamplona members might reflect local fluctuations in the availability of these elements due to its coeval precipitation with pyrite, hematite and/or sphalerite. This alteration is marked by higher concentrations of $\mathrm{Na}_{2} \mathrm{O}, \mathrm{SiO}_{2}, \mathrm{Sr}, \mathrm{Ba}, \mathrm{Cu}, \mathrm{Ni}, \mathrm{As}, \mathrm{Re}$, and $\mathrm{S}$.

Late quartz from the pyrite-bearing alteration (quartz 4; Fig. 2.7) in the Lower Pamplona Member yielded the similar types of $\mathrm{H}_{2} \mathrm{O}-\mathrm{NaCl}$-bearing fluid inclusions as quartz (quartz 3; Fig 2.7) associated to the latest phase of the ore stage (Table 2.6). However, quartz 4 in the Upper Morro do Pinheiro Member hosts a third type of fluid inclusion in this alteration style, composed of $\mathrm{H}_{2} \mathrm{O}-\mathrm{NaCl}-\mathrm{CaCl}_{2}$, of moderate salinity (7.3 to $14.6 \mathrm{wt} . \% \mathrm{NaCl}$ equiv.) and homogenization temperatures of $124^{\circ}$ to $152^{\circ} \mathrm{C}$. The calculated isotopic compositions of the fluids associated with dolomite is similar to the ones calculated to red and white dolomite from the ore stage. These data and textural relationships (Fig. 2.12) suggest that the pyrite-bearing alteration style formed by similar fluids to the ore stage alteration. Sulfide precipitation was favorable in sites where reduced sulfur was available, however willemite formed in S-poor sites by mixture of metallifereous (higher temperature and salinity fluids) with evolved meteoric water (lower temperature and salinity), consistent with what was previously proposed by Monteiro et al., 2006 and Slezak et al., 2014. Coeval precipitation of pyrite and hematite from the pyrite-bearing alteration close to the ore stage alteration halo in the Lower Pamplona Member reflects fluctuations in the $f \mathrm{O}_{2} / f \mathrm{~S}_{2}$ conditions, supporting mixing with descendent more oxidizing fluids (evolved meteoric water). In occurrences where ore stage and early sulfide alterations are poorly developed (saddle, red and white dolomite from early phases of the ore stage as well as white dolomite from the early phases of the pyrite bearing sulfide alteration), the $\mathrm{C}$ and $\mathrm{O}$ isotopic composition of the fluids responsible for precipitation of dolomite were buffered by the host dolostones composition (although saddle dolomite show lighter $\mathrm{C}$ composition). Where these alterations are pervasive, indicating higher fluid/rock ratio, the $\mathrm{C}$ and $\mathrm{O}$ isotopic composition of the fluids are isotopically lighter (Fig. 2.23-B).

The galena-bearing alteration characterized by sphalerite, white dolomite $(\sim 2.5 \%$ in average $\mathrm{Fe}$ ) and traces of galena, chalcocite, greenockite and covellite, which was identified only in the Lower Pamplona Member, is tentatively correlated with the late sulfide veinlets described by Slezak et al. (2014). These veinlets cut both the willemite main stage ore and the early pyrite-style alteration, suggesting a later hydrothermal event with distinct metal signature.

The latest event, which was observed only in one sample with galena-bearing alteration style, is characterized by silicification producing willemite by reaction of sphalerite with quartz. 
Higher content of $\mathrm{Cd}$ and $\mathrm{Pb}$ in willemite from this stage is interpreted to be due to the higher concentrations of these chalcophile elements in the precursor sphalerite in the Lower Pamplona Member (411.13 m). Fluid inclusions associated with this alteration were not identified.

\subsubsection{Comparison with other Vazante District Zn deposits}

The characteristics of the willemite ore zones hosted in the Upper Morro do Pinheiro and the Lower Pamplona members intercepted in the drill hole VZVG003 are similar to the hypogene willemitic mineralizations described in the Vazante Mine (Monteiro et al., 2006), Vazante Northern Extension (Slezak et al., 2014) and the Olho D'água, Cercado and Pamplona occurrences (Baia, 2013; Table 2.1), indicating that similar processes were related to the formation of the willemite in these sites. Significantly, the fluids reported by Dardenne and Freitas-Silva (1999) for the Vazante deposit are of low to moderate salinities and temperatures, similar to the fluids (Type 2.4; Table 2.6; Fig. 2.32) associated to the late phases of the willemite ore stage. These fluids are interpreted to represent the contribution of evolved meteoric water mixing with metalliferous brines in the formation of willemite mineralization. As they are also found in late quartz from the pyrite-bearing alteration, it suggests that mixing of these fluids was also involved in the sites of pyrite precipitation, where reduced sulfur was available.

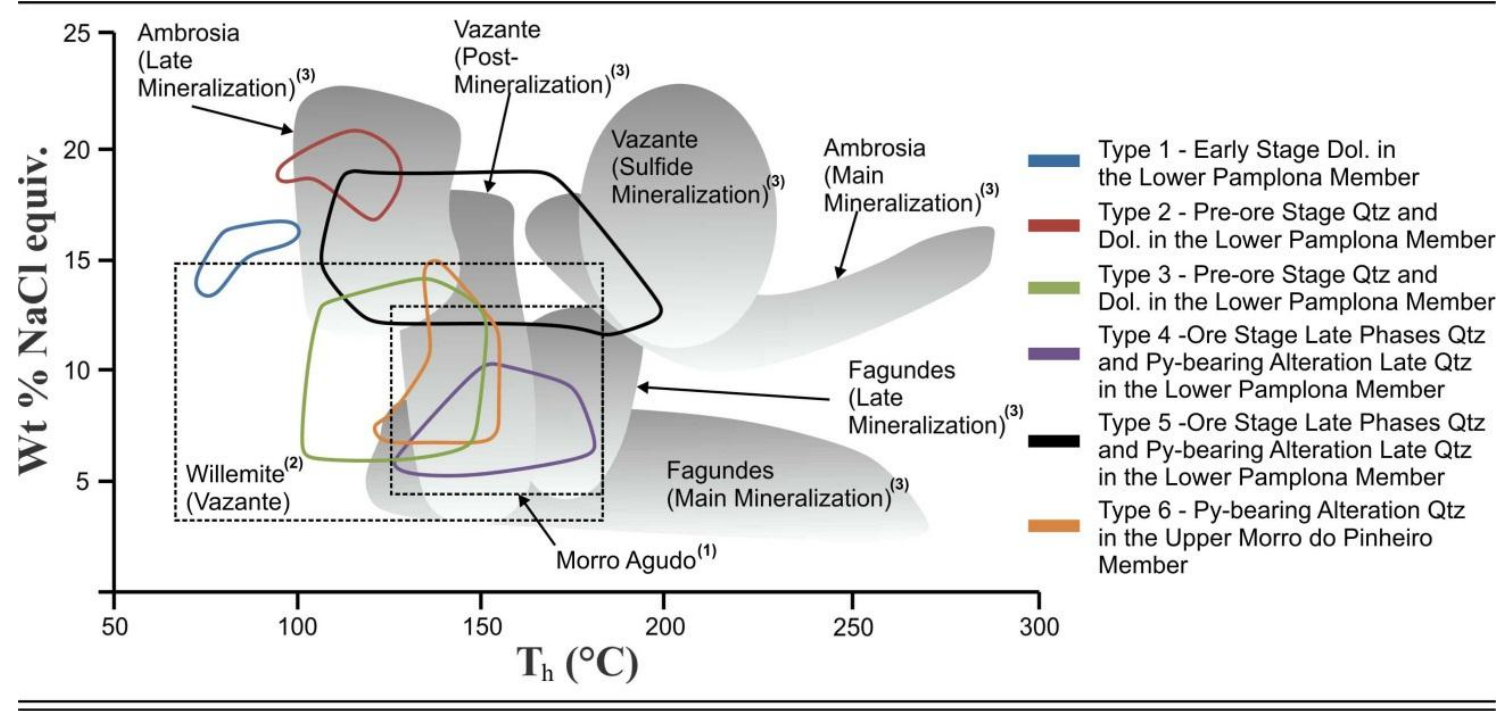

(1) Cunha, et al., 2000; (2) Dardenne and Freitas-Silva, 1999; (3) Monteiro, 2002.

Fig. 2.32. Comparison of homogenization temperatures $\left(\mathrm{T}_{\mathrm{h}}\right)$ and salinities (wt. \% $\mathrm{NaCl}$ equiv.) of fluid inclusions from this study with other studies in different deposits.

The high salinity hydrothermal fluids (Type 5; Table 2.6; Fig. 2.32) associated to the late phases of the ore stage as well as to late quartz from the pyrite-bearing alteration have similar compositions and average homogenization temperature to the fluids documented by Cunha et al., 2000 in the sulfide-rich ore in the Morro Agudo deposit. They are tentatively interpreted as the metalliferous brines. The salinities of the fluids involved in the sulfide mineralizations in Ambrósia and Vazante deposits (Monteiro, 2002) are also similar, although they revealed higher homogenization temperatures (up to $281^{\circ}$ and $232^{\circ} \mathrm{C}$, respectively), indicating either lower input of cooler meteoric water and/or that they were more proximal to the metalliferous brine conduits. The salinites of the $\mathrm{H}_{2} \mathrm{O}-\mathrm{NaCl}-\mathrm{CaCl}_{2}$ fluids (Type 6, Table 2.6) associated to the pyrite-bearing alteration in the Upper Morro do Pinheiro Member are more similar to fluids reported in the sulfide mineralization of the Fagundes deposit (Monteiro, 
2002), however the latter yielded higher homogenization temperatures (up to $260^{\circ} \mathrm{C}$ ). These fluids may represent $\mathrm{Ca}$ contribution from the dissolution of the carbonate rocks. Sphalerite from pyrite-bearing style of alteration in the Upper Morro do Pinheiro Member shows trace elements composition similar to sphalerite from the Ambrósia deposit, whereas sphalerite from the galena-bearing style of alteration in the Lower Pamplona Member is more alike sphalerite from the Fagundes deposit (Fig. 2.18). Therefore the relationship between these two styles of sulfide alteration in the Vazante district and Ambrósia and Fagundes sulfide deposits in the Paracatu region needs to be better investigated.

The $\mathrm{C}$ and $\mathrm{O}$ compositions of the carbonates associated with both sulfide ore in the Paracatu and Vazante regions and silicate zinc ore in the Vazante region are lighter than the host rock isotopic composition. The lighter $\mathrm{O}$ isotopic composition has been interpreted to be due to the light composition of the metalliferous brine (due to lighter $\mathrm{O}$ isotopic composition in the pyrite-bearing alteration) and/or mixture with evolved meteoric water (Monteiro et al., 2007; Olivo et al., 2013). The lighter O isotopic composition of carbonates coeval to willemite formation supports the hypothesis that mixing of metalliferous fluids with light $\mathrm{O}$ isotopic composition fluids (evolved meteoric water) was determinant for hypogene zinc silicate precipitation. Organic matter or hydrocarbons in the rocks that interacted with the hydrothermal fluids, or bacteriological processes, might have been involved in willemite precipitation due to the lighter $\mathrm{C}$ isotopic composition of carbonates coeval to willemite, although this interaction with organic carbon did not supplied sulfur in the Southern Extension of the Vazante Group, due to the very low content of this element in the mineralized zones. As carbonic fluids were not identified in the fluid inclusions in this and previous studies, the lighter $\mathrm{C}$ isotopic composition may be associated with involvement of hydrocarbons or organic matter during the mineralizing stages. The role of organic carbon would have been more relevant in the sulfide ore, which yielded lighter $\mathrm{C}$ values (Table 2.3). This suggests that, in these cases, organic matter is related to the source of reduced sulfur.

The similarities between the fluid compositions (fluid inclusions and isotopes) between the South and North sectors of the Vazante Group during the zinc mineralizing event suggest that these fluids percolated throughout the Vazante Group and are related to the formation of both sulfide $\mathrm{Pb}-\mathrm{Zn}$ and silicate zinc deposits. The metalliferous saline fluids may have transported $\mathrm{Zn}$ as chlorite complexes $\left(\mathrm{ZnCl}_{4}^{-2}\right)$ and $\mathrm{Zn}$ precipitated as willemite where the metalliferous fluids interacted with more oxidizing and alkaline fluids, however sphalerite was the dominant ore mineral where reduced sulfur was available at the deposition site (Brugger et al., 2003; Slezak et al., 2014).

\subsection{CONCLUSIONS}

The major findings of this study are:

- There are multiple stages of fluid-rock interaction during the evolution of the Southern Extension of the Vazante Group.

- The earliest diagenetic/hydrothermal event, which is characterized by formation of dolomite substitution bands, is interpreted to have been formed during late diagenesis at temperatures around $90^{\circ} \mathrm{C}$ by moderate salinities fluids of $\mathrm{H}_{2} \mathrm{O}-\mathrm{NaCl}-\mathrm{CaCl}_{2}$ composition with very light $\mathrm{C}$ and $\mathrm{O}$ isotopic composition, suggesting meteoric and/or connate water origin with organic carbon and evaporites interaction. They are very distinct of the fluids associated with the base metal mineralizing events. 
- The pre-ore stage hydrothermal event, which comprises mainly dolomite and quartz filling dissolution voids, are associated with mixing of two types of fluids $\left(\mathrm{H}_{2} \mathrm{O}-\mathrm{NaCl}-\right.$ $\mathrm{CaCl}_{2}$ composition, 18.0 to $20.3 \mathrm{wt} \% \mathrm{NaCl}$ equiv., $\mathrm{T}_{\mathrm{h}}$ of $98^{\circ}$ to $125^{\circ} \mathrm{C}$ and $\mathrm{H}_{2} \mathrm{O}-\mathrm{NaCl}-$ $\mathrm{MgCl}_{2}$ composition, 6.4 to $13.7 \mathrm{wt} \% \mathrm{NaCl}$ equiv., $\mathrm{T}_{\mathrm{h}}$ of $105^{\circ}$ to $148^{\circ} \mathrm{C}$ ); The isotopic compositions of $\mathrm{O}$ and $\mathrm{C}$ in of these fluids are heavier than the earliest fluids and lighter than the average composition of the fluids associated with the subsequent stages of alteration.

- The ore stage alteration comprises four phases: (1) the earliest characterized distal reddish dolostone with higher concentrations of $\mathrm{Al}_{2} \mathrm{O}_{3}, \mathrm{P}_{2} \mathrm{O}_{5}$, and some rare earth elements (REE: Ce, Dy, Er, Eu, Ga, Gd, Nd, Pr, Sm, Tb and Y), and discrete higher concentrations of $\mathrm{Ni}, \mathrm{Pb}$ and $\mathrm{As}$; (2) the main ore stage phase which comprises abundant red dolomite, hematite and willemite and is characterized by higher concentrations of $\mathrm{SiO}_{2}, \mathrm{Fe}_{2} \mathrm{O}_{3}, \mathrm{P}_{2} \mathrm{O}_{5}, \mathrm{Mn}, \mathrm{As}, \mathrm{Ge}, \mathrm{Hg}$, In, U, V, W, Ag, Cd, Cu, Mo, Ni, $\mathrm{Pb}, \mathrm{Sb}, \mathrm{Se}$, and $\mathrm{Zn}$; (3) the third is represented by white dolomite, hematite and traces of willemite with higher concentrations of $\mathrm{Mn}, \mathrm{Ba}, \mathrm{Cr}, \mathrm{Cd}, \mathrm{Ni}, \mathrm{Pb}$, and $\mathrm{As}$, which cross-cut the main ore stage; (4) the latest phase comprises $\mathrm{Zn}$-chlorite and quartz which were precipitated by the mixture of a higher temperature and salinities fluids with lower temperature and salinities fluids $\left(\sim 170^{\circ}\right.$ to $190^{\circ} \mathrm{C} / 12.2$ to 18.4 wt. $\% \mathrm{NaCl}$ equiv. and $\sim 90^{\circ}$ to $130^{\circ} \mathrm{C} / 5.8$ to $9.9 \mathrm{wt} \% \mathrm{NaCl}$ equiv., respectively). The composition of the higher salinity and higher temperature fluids is $\mathrm{H}_{2} \mathrm{O}-\mathrm{NaCl}$.

- The mineralization in the Upper Morro do Pinheiro and Lower Pamplona members exhibit similar characteristics, with higher concentrations of $\mathrm{SiO}_{2}, \mathrm{Fe}_{2} \mathrm{O}_{3}, \mathrm{P}_{2} \mathrm{O}_{5}, \mathrm{Mn}$, As, $\mathrm{Ge}, \mathrm{Hg}$, In, U, V, W, Ag, Cd, Cu, Mo, Ni, Pb, Sb, Se, and Zn.; although the former yielded higher $\mathrm{Zn}$ and $\mathrm{Cu}$ grades, and the latter has higher $\mathrm{Pb}, \mathrm{Mn}$, As, and $\mathrm{V}$ grades and is anomalous in $\mathrm{Sb}$.

- The mineralization is characterized by lighter $\mathrm{C}$ and $\mathrm{O}$ isotopic composition. The lower $\mathrm{O}$ isotopic values might reflect a lighter composition of the metalliferous fluid but also might be related to mixture of the metalliferous fluid with evolved meteoric water. This data are consistent with the model that the silicate zinc ore formed by mixing between of an ascendant metalliferous fluid and more oxidizing, more alkaline evolved meteoric water.

- The sulfide-bearing alteration stages include: (1) pyrite-bearing alteration represented by pyrite, sphalerite, white dolomite, fine hematite and late quartz. This alteration shows higher concentrations of $\mathrm{Na}_{2} \mathrm{O}, \mathrm{SiO}_{2}, \mathrm{Sr}, \mathrm{Ba}, \mathrm{Cu}, \mathrm{Ni}, \mathrm{As}, \mathrm{Re}$, and $\mathrm{S}$, and the dolomite isotopic composition is similar to the composition of red dolomite and white dolomite from the main phase of the ore stage and from the third phase of the ore stage, respectiveky, suggesting the same metalliferous source for this alteration styles. Late quartz from this alteration evidences mixture of the same fluids involved in late quartz precipitation of the ore stage, indicating similar genetic relationships for these mineral phases among these two alteration styles; (2) galena-bearing alteration comprised by sphalerite, galena, chalcocite, greenockite, covellite and white dolomite, which crosscut the mineralized bodies and is interpreted to be late to the pyrite-bearing alteration.

- The sites with sulfide-rich mineralization have more significant input of organic matter or hydrocarbons which may have provided the reduced sulfur.

- The similarities between the fluid compositions (fluid inclusions and isotopes) between the South and North sectors of the Vazante Group during the zinc mineralizing event 
suggest that these fluids percolated throughout the Vazante Group and are related to the formation of both sulfide $\mathrm{Pb}-\mathrm{Zn}$ and silicate zinc deposits. 


\section{Capítulo 3}

\subsection{DISCUSSÕES}

\subsubsection{Evolução do sistema hidrotermal na Extensão Sul do Grupo Vazante}

O estudo da Extensão Sul do Grupo Vazante revelou vários estágios de alteração hidrotermal, os quais são sumarizadas nas figuras 2.6 e 2.25. O evento hidrotermal mais precoce documentado no Grupo Vazante é caracterizado por bandas de substituição de dolomita preferencialmente subparalelas às laminações sedimentares. Essa dolomita é pobre em elementos traço e exibe discretos valores mais elevados em Fe, $\mathrm{Mn}$ e $\mathrm{Cd}$ (Tabela 2.4; Figura 2.15). Os fluidos associados a esse evento são compostos por $\mathrm{H}_{2} \mathrm{O}-\mathrm{NaCl}-\mathrm{CaCl}_{2}$, com salinidades entre 13,8 a 16,2\% em peso equiv. de $\mathrm{NaCl}$ (Tabela 2.6; Figura 2.21) e temperaturas de homogeneização entre $75^{\circ}$ e $97^{\circ} \mathrm{C}$ (Tabela 2.6; Figura 2.22). As composições isotópicas de $\mathrm{C}$ e $\mathrm{O}$ calculadas para esses fluidos são mais leves que os fluidos dos eventos subsequentes. Esta alteração hidrotermal é interpretada como resultante de processos de dissolução de carbonato e reprecipitação de dolomita durante a tardi-diagênese. As salinidades moderadas dos fluidos assim como as composições isotópicas de $\mathrm{C}$ e $\mathrm{O}$ sugerem que os fluidos eram de origem meteórica e/ou conata, que interagiram com evaporitos assim como com matéria orgânica ou hidrocarbonetos, todos estes relatados no Membro Pamplona Inferior (Dardenne et al., 1998; Oliveira 2013; Slezak et al., 2014).

O segundo evento hidrotermal (estágio pre-mineralização) é caracterizado por dolomita dog-tooth e blocky e quartzo, precipitados em cavidades de dissolução. Essa alteração foi desenvolvida por mistura de fluidos, indicada pela presença de fluidos de composição $\mathrm{H}_{2} \mathrm{O}$ $\mathrm{NaCl}-\mathrm{CaCl}_{2}$, com salinidades entre 18,0 a $20,3 \%$ em peso de $\mathrm{NaCl}$ equiv. e temperaturas de homogeneização entre $98^{\circ}$ e $125^{\circ} \mathrm{C}$ e fluidos de composição $\mathrm{H}_{2} \mathrm{O}-\mathrm{NaCl}-\mathrm{MgCl}_{2}$, com salinidades de 6,4 a 13,7\% em peso de $\mathrm{NaCl}$ equiv. e temperaturas de homogeneização entre $105^{\circ}$ e $148^{\circ} \mathrm{C}$ (Tabela 2.6). As composições isotópicas de carbono calculadas para os fluidos em equilíbrio com dolomita é de $-1,81 \%$ a $-1,04 \%$, que são mais pesadas que as composições dos fluidos do estágio precoce, mas são, em média, mais leves que as fases subsequentes, o que suporta a interação com carbone leve. Esta alteração exibe valores mais elevados em $\mathrm{SiO}_{2}$ e é empobrecida em elementos relacionados à mineralização, o que indica que esses fluidos não interagiram com os fluidos mineralizantes.

As alterações hidrotermais relacionadas ao minério de zinco silicatado compreende três fases principais. A primeira é marcada por ocorrência de dolomitos avermelhados distais, documentados principalmente no Membro Pamplona Inferior. Essa fase apresenta assinatura geoquímica de valores mais elevados em $\mathrm{Al}_{2} \mathrm{O}_{3}, \mathrm{P}_{2} \mathrm{O}_{5}$, e alguns elementos de terras raras (ETR's: Ce, Dy, Er, Eu, Ga, Gd, Nd, Pr, Sm, Tb e Y), com discretos valores mais elevados em $\mathrm{Ni}, \mathrm{Pb}$ e As. Inclusões fluidas não foram identificadas nestas rochas alteradas. Apesar desta alteração comumente ocorrer como halos distais em zonas externas ao redor dos núcleos mineralizados, sua assinatura geoquímica é bastante distinta. Sua ligação genética com a alteração mineralizante, por tanto, não é clara.

A segunda fase do estágio mineralizante é o estágio mineralizante principal e compõe as zonas de alto teor de Zn nos membros Morro do Pinheiro Superior (480 a 570 m) e Pamplona Inferior (48 a $210 \mathrm{~m}$ ). Esse estilo de alteração é tipicamente representado por brechas de dissolução com maciça dolomita vermelha zincífera (médias de $\sim 1 \%$ de $\mathrm{Zn}, \sim 0,5 \%$ de $\mathrm{Fe}$ ), hematita abundante e willemita $(\sim 0,25 \%$ de $\mathrm{Fe}$, em média). Essa alteração forma corpos 
maciços no intervalo mineralizado do Membro Morro do Pinheiro Superior e arranjos mais disseminados no Membro Pamplona Inferior. Brechas hidráulicas são observadas principalmente na zona mineralizada do Membro Pamplona Inferior (Figura 2.6), o que sugere que essas brechas se formaram em zonas mais rasas, com pressão litostática reduzida e/ou pressões de fluidos hidrotermais mais altas. A assinatura geoquímica global do estágio principal de mineralização é caracterizada por valores mais elevados em $\mathrm{SiO}_{2}, \mathrm{Fe}_{2} \mathrm{O}_{3}, \mathrm{P}_{2} \mathrm{O}_{5}, \mathrm{Mn}$, As, Ge, $\mathrm{Hg}$, In, U, V, Ag, Cd, Cu, Mo, Ni, Pb, Sb, Se e Zn. No Membro Morro do Pinheiro Superior, há valores mais elevados também de Co, e mais altas concentrações de Cu e Zn (até 39,8\% de Zn contra 33,9\% de Zn no membro sobreposto). A zona mineralizada do Membro Pamplona Inferior apresenta, também, valores anômalos de $\mathrm{Sb}$ e maiores teores de $\mathrm{As}, \mathrm{Mn}, \mathrm{Pb}$ e V.

A terceira fase relacionada ao estágio mineralizante é constituída por dolomita branca, com menores concentrações de hematita e willemita e é caracterizada por valores mais elevados em $\mathrm{Mn}, \mathrm{Ba}, \mathrm{Cr}, \mathrm{Cd}, \mathrm{Ni}, \mathrm{Pb}$ e As. As assinaturas isotópicas de carbono e oxigênio dos carbonatos das segunda e terceira fases são similares $(+0.26$ a $+1.23 \% 0 /+19.39$ a $+22.82 \%$ e -1.62 a $+1.41 \%$ / $+16.46 \mathrm{a}+21.51 \%$, respectivamente) e composições isotópicas de carbono mais leves são encontradas em dolomita da segunda fase (principal estágio mineralizante), coeva a willemita (Figura 2.23-A). Isso aponta que em locais de precipitação de willemita, mistura de fluidos com composições isotópicas de carbono e oxigênio mais leves foi realçada. Composições isotópicas de oxigênio mais leves devem estar relacionadas ao envolvimento de água meteórica, enquanto composições isotópicas mais leves de carbono sugerem que os fluidos hidrotermais interagiram com rochas carbonosas ou que processos bacteriológicos estavam envolvidos durante essa fase hidrotermal. Esses fluidos eram deficientes em enxofre (conteúdos abaixo do limite de detecção do método geoquímico), o que indica que a interação com carbono orgânico não forneceu enxofre ao sistema. Inclusões fluidas não foram obtidas para essas fases.

A quarta fase relacionada ao estágio mineralizante é representada por quartzo (quartz 3, Figura 2.7) e clorita zincífera e exibe dois tipos de inclusões fluidas, com salinidades distintas ( 5,8 a $9,9 \%$ e 12,2 a $18,4 \%$ em peso de $\mathrm{NaCl}$ equiv.) e de grande variabilidade de temperaturas de homogeneização $\left(90^{\circ}\right.$ a $178^{\circ} \mathrm{C}$ e $92^{\circ}$ a $196^{\circ} \mathrm{C}$, respectivamente). As inclusões de maior salinidade apresentam maior correlação com temperaturas de homogeneização mais altas (Figura 2.32; altos valores de $\mathrm{T}_{\mathrm{h}}$ das inclusões fluidas do tipo 5 são de mais alta salinidade do que as inclusões do tipo 4). As inclusões de mais baixa salinidade apresentam temperatura de eutético típicas do sistema salino $\mathrm{H}_{2} \mathrm{O}-\mathrm{NaCl}$ e temperaturas de primeira fusão de gelo não foram observadas na inclusões fluidas de menor salinidade. Esses dados suportam a hipótese de que a mistura de fluidos de mais altas salinidades e temperatura $\left(\sim 170^{\circ}\right.$ a $\left.190^{\circ} \mathrm{C}\right)$ com um fluido de mais baixas salinidades e de mais baixa temperatura $\left(\sim 90^{\circ}\right.$ a $\left.130^{\circ} \mathrm{C}\right)$ ocorreu durante a fase tardia da alteração hidrotermal mineralizante.

A alteração hidrotermal de pirita, representada pela assembleia pirita, esfalerita, dolomita branca, quartzo e hematita fina, revelou conteúdos de $\mathrm{FeO}$ em dolomita branca no membros Morro do Pinheiro Superior e Pamplona Inferior em média de $1.389 \%$ e $2.474 \%$, respectivamente (Tabela 2.4). Variações nos conteúdos de $\mathrm{FeO}$ e $\mathrm{Zn}$ em dolomita da alteração de pirita nos membros Morro do Pinheiro Superior e Pamplona Inferior devem refletir flutuações locais na disponibilidade desses elementos devido a co-precipitação com pirita, hematita e/ou esfalerita. Essa alteração é marcada pelos valores mais elevados em $\mathrm{Na}_{2} \mathrm{O}, \mathrm{SiO}_{2}$, $\mathrm{Sr}, \mathrm{Ba}, \mathrm{Cu}, \mathrm{Ni}, \mathrm{As}, \mathrm{Re}$ e S.

Quartzo tardio da alteração de pirita (quartz 4; Figura 2.7) no Membro Pamplona Inferior hospeda inclusões fluidas similares às inclusões fluidas tipo 4 e tipo 5 (Tabela 2.6) identificadas em quartzo da fase tardia do estágio mineralizante (quartz 3; Figura 2.7). Quartzo tardio da alteração de pirita (quartz 4; Figura 2.7) no Membro Morro do Pinheiro Superior, no 
entanto, hospeda um terceiro tipo de inclusões fluidass para essa alteração, composta por $\mathrm{H}_{2} \mathrm{O}$ $\mathrm{NaCl}-\mathrm{CaCl}_{2}$, de salinidade moderada (7,3 a $14,6 \%$ em peso de $\mathrm{NaCl}$ equiv.) e temperaturas de homogeneização entre $124^{\circ}$ e $152^{\circ} \mathrm{C}$. As composições isotópicas de $\mathrm{C}$ e $\mathrm{O}$ dos fluidos associados a dolomita é similar às composições dos fluidos associados a dolomitas vermelha $\mathrm{e}$ branca do estágio mineralizante. Esses dados e relações texturais (Figura 2.12) sugerem que a alteração de pirita foi formada por fluidos similares aos fluidos associados ao estágio mineralizante. Precipitação de sulfetos foi favorecida em locais onde enxofre reduzido estava disponível, enquanto willemita se formou em sítios pobres em enxofre pela mistura de fluidos metalíferos (mais alta temperatura e salinidade) com água meteórica evoluída (menor temperatura e salinidade), hipótese consistente com a previamente proposta por Monteiro et al., (2006) e Slezak et al., (2014). Coprecipitação de pirita e hematita da alteração de pirita próxima ao halo de alteração do estágio mineralizante no Membro Pamplona Inferior reflete flutuações nas condições de $f \mathrm{O}_{2} / f \mathrm{~S}_{2}$, o que apoia a hipótese de mistura com fluidos descendentes mais oxidantes (água meteórica evoluída). Em ocorrências onde as alterações relacionadas ao estágio mineralizante e à alteração de pirita são pouco desenvolvidas (dolomita em cela e vermelha das fases precoces do estágio mineralizante assim como dolomita branca das fases iniciais da alteração de pirita), a composição isotópica do fluido em equilíbrio com dolomita foi tamponada pela composição dos dolomitos hospedeiros (embora dolomita em cela apresente composição isotópica de carbono mais leve). Onde essas alterações são mais pervasivas, que indica maior razão fluido/rocha, as composição isotópicas de $\mathrm{C}$ e $\mathrm{O}$ dos fluidos é mais leve (Figura 2.23-B).

A alteração de galena é caracterizada por esfalerita, dolomita branca $(\sim 2.5 \%$ de $\mathrm{Fe}$, em média) e traços de galena, calcocita, greenockita e covelita, e foi identificada somente no Membro Pamplona Inferior. Essa alteração é tentativamente correlacionada a veios de sulfetos tardios descritos por Slezak et al., (2014). Esses veios cortam ambos estágio mineralizante willemítico e alteração de pirita precoce, o que sugere que a alteração de galena represente uma alteração posterior com assinatura química distinta. O evento hidrotermal mais tardio identificado foi observado somente em uma amostra e é caracterizado por silicificação que produz willemita pela reação de quartzo com esfalerita. Maiores concentrações de $\mathrm{Cd}$ e $\mathrm{Pb}$ em willemita desse estágio são interpretadas como sendo devido aos valores mais elevados desses elementos calcófilos em esfaleritas precursoras no Membro Pamplona Inferior (411,13 m). Inclusões fluidas associadas a essa alteração não foram identificadas.

\subsubsection{Comparação com outros depósitos de Zn no Distrito de Vazante}

As características das zonas de mineralização willemítica hospedadas nos membros Morro do Pinheiro Superior e Pamplona Inferior interceptados no poço VZVG003 são similares às descritas para as mineralizações de willemita hipogênica nas minas de Vazante (Monteiro et al., 2006) e Extensão Norte (Slezak et al., 2014) e nas ocorrências do Olho D'água, Cercado and Pamplona (Baia, 2013; Tabela 2.1). Isso indica que processos similares foram relatados para a formação de willemita nesses locais. Os fluidos relatados por Dardenne e Freitas-Silva (1999) para o depósito de Vazante são de salinidades baixas a moderadas, similares aos fluidos associados às fases tardias do estágio de mineralização willemítica (tipo 4; Tabela 2.6; Figura 2.26). Esses fluidos são interpretados como sendo produto de mistura de água meteórica evoluída com salmouras metalíferas em zonas de formação de willemita. Esses fluidos também são encontrados em quartzo tardio da alteração de pirita, o que sugere que processos de mistura de fluidos também ocorreram em sítios de precipitação de pirita, onde enxofre reduzido estava disponível. 
A alta salinidade dos fluidos hidrotermais associados à fase tardia do estágio mineralizante assim como a quartzo tardio da alteração de pirita (inclusões fluidas tipo 5; Tabela 2.6) apresentam composições e temperaturas de homogeneização médias similares aos fluidos documentados por Cunha et al., (2000) no depósito sulfetado de Morro Agudo.

Esses fluidos são tentativamente interpretados como representantes das salmouras metalíferas. As salinidades dos fluidos envolvidos nas mineralizações sulfetadas nos depósitos de Ambrósia e Vazante (Monteiro, 2002) também são similares àqueles fluidos, embora esses revelem temperaturas de homogeneização mais altas (até $281^{\circ} \mathrm{C}$ e $232^{\circ} \mathrm{C}$, respectivamente), o que indica menor introdução de água meteórica mais fria e/ou esses depósitos eram mais próximos dos condutos das salmouras metalíferas. As salinidades dos fluidos tipo 6 (Tabela 2.6) compostos por $\mathrm{H}_{2} \mathrm{O}-\mathrm{NaCl}-\mathrm{CaCl}_{2}$ associados à alteração de pirita no Membro Morro do Pinheiro Superior são similares às salinidades dos fluidos relatadas no depósito de Fagundes, associados à mineralização sulfetada (Monteiro, 2002). Esses fluidos, no entanto, exibem temperaturas de homogeneização mais altas (até $260^{\circ} \mathrm{C}$ ). Ambos fluidos devem representar a contribuição de $\mathrm{Ca}$ a partir de dissolução das rochas carbonáticas hospedeiras. A mais alta temperatura dos fluidos do depósito de Fagundes é intrigante, uma vez que a mineralização aparentemente se formou em um nível estrutural mais raso do que outros depósitos sulfetados e silicatado (dados de exploração da Votorantim Metais). Esfalerita da alteração de pirita no Membro Morro do Pinheiro Superior mostra composição de elementos traço similar a esfalerita do depósito de Ambrósia, enquanto que a composição de esfalerita da alteração de galena no Membro Pamplona Inferior é similar a esfalerita do depósito de Fagundes (Figura 2.18). A relação entre esses dois estilos de alteração sulfetada no distrito de Vazante e nos depósitos sulfetados de Ambrósia e Fagundes na região de Paracatu, portanto, precisa ser melhor investigada.

As composições isotópicas de $\mathrm{C}$ e $\mathrm{O}$ dos carbonatos associados com ambos minérios sulfetados nas regiões de Paracatu e Vazante e minério silicatado na região de Vazante são mais leves que as composições das rochas hospedeiras. As composições mais leves tem sido interpretadas como sendo devido às composições isotópicas mais leves da salmoura metalífera (devido às composições isotópicas de $\mathrm{O}$ mais leves na alteração de pirita) e/ou mistura com água meteórica evoluída (Monteiro et al., 2007; Olivo et al., 2013). As composições isotópicas mais leves de $\mathrm{O}$ dos carbonatos em equilíbrio com formação de willemita dá suporte à hipótese de que a mistura de fluidos metalíferos com fluidos de composições isotópicas mais leves de $\mathrm{O}$ (água meteórica evoluída) foi determinante para a precipitação hipogênica de zinco silicatado. As composições isotópicas mais leves de $\mathrm{C}$ em dolomita em equilíbrio com willemita sugere que os fluidos hidrotermais responsáveis pela precipitação dessas fases minerais interagiu com matéria orgânica ou hidrocarbonetos das rochas encaixantes ou processos bacteriológicos estavam envolvidos na precipitação de willemita. Essa potencial interação com matéria orgânica, no entanto, não forneceu enxofre para o sistema na Extensão Sul do Grupo Vazante, devido ao conteúdo muito baixo desse elemento nas zonas mineralizadas. Como fluidos carbônicos não foram identificados em inclusões fluidas neste e em estudos anteriores, a composição isotópica mais leve de $\mathrm{C}$ deve estar associada ao envolvimento de hidrocarbonetos e matéria orgânica durante os estágios mineralizantes. O papel da matéria orgânica seria mais relevante no minério sulfetado, o qual revelou os valores mais leves de isótopos de $\mathrm{C}$ (Tabela 2.3). Isso sugere que, nestes casos, matéria orgânica está relacionada à fonte de enxofre reduzido.

Os fluidos salinos metalíferos devem ter transportado $\mathrm{Zn}$ como complexos cloretados $\left(\mathrm{ZnCl}_{4}{ }^{-2}\right)$ e o $\mathrm{Zn}$ precipitou como willemita em locais onde os fluidos metalíferos interagiram com fluidos mais oxidantes e alcalinos. Esfalerita é o mineral minério dominante onde enxofre estava disponível nos sítios de precipitação (Brugger et al., 2003; Slezak et al., 2014). 
E importante ressaltar que os fluidos mais precoces, interpretados como diagenéticos, tem uma composição e temperaturas muito distintas dos fluidos associados aos eventos mineralizadores de metais de base.

\subsection{CONCLUSÕES}

As maiores conclusões deste estudo são:

- Múltiplos estágios de interação fluido-rocha ocorreram durante a evolução da Extensão Sul do Grupo Vazante.

- O evento hidrotermal mais precoce, o qual é caracterizado pela formação de bandas de substituição de dolomita, é interpretado como tendo sido formado durante a tardidiagênese, a temperaturas em torno de $90^{\circ} \mathrm{C}$, por fluidos de salinidades moderadas e composição $\mathrm{H}_{2} \mathrm{O}-\mathrm{NaCl}-\mathrm{CaCl}_{2}$. Esses fluidos apresentavam composição isotópica bastante leve de $\mathrm{C}$ e $\mathrm{O}$, o que sugere origem meteórica ou conata para o fluido que interagiu com carbono orgânico e evaporitos.

- O evento hidrotermal do estágio pré-mineralização, o qual compreende, principalmente, dolomita e quartzo que preenchem cavidades de dissolução, são associados à mistura de dois fluidos. Um composto por $\mathrm{H}_{2} \mathrm{O}-\mathrm{NaCl}-\mathrm{CaCl}_{2}$, de salinidades entre 18,0 a 20,3\% em peso de $\mathrm{NaCl}$ equiv. e $\mathrm{T}_{\mathrm{h}}$ entre $98^{\circ}$ a $125^{\circ} \mathrm{C}$ e outro composto por $\mathrm{H}_{2} \mathrm{O}-\mathrm{NaCl}-\mathrm{MgCl}_{2}$, de salinidades entre 6,4 a $13,7 \%$ em peso de $\mathrm{NaCl}$ equiv. e $\mathrm{T}_{\mathrm{h}}$ de $105^{\circ}$ a $148^{\circ} \mathrm{C}$. As composições isotópicas de $\mathrm{C}$ e $\mathrm{O}$ destes fluidos são mais pesadas que as dos fluidos do evento mais precoce, porém é, em geral, mais leve que os fluidos envolvidos nos eventos subsequentes de alteração hidrotermal.

- A alteração hidrotermal do estágio mineralizante compreende quatro fases: (1) a primeira é caracterizada por valores mais elevados em $\mathrm{Al}_{2} \mathrm{O}_{3}, \mathrm{P}_{2} \mathrm{O}_{5}$ e em alguns elementos de terras raras (ETR's: Ce, Dy, Er, Eu, Ga, Gd, Nd, Pr, Sm, Tb e Y) e discretos valores mais elevados em $\mathrm{Ni}, \mathrm{Pb}$ e As; (2) o estágio principal de mineralização corresponde a abundante dolomita vermelha, hematita e willemita e é caracterizada por valores mais elevados em $\mathrm{SiO}_{2}, \mathrm{Fe}_{2} \mathrm{O}_{3}, \mathrm{P}_{2} \mathrm{O}_{5}, \mathrm{Mn}$, As, Ge, $\mathrm{Hg}$, In, U, V, W, Ag, Cd, $\mathrm{Cu}$, $\mathrm{Mo}, \mathrm{Ni}, \mathrm{Pb}, \mathrm{Sb}, \mathrm{Se}$ e $\mathrm{Zn}$; (3) a terceira fase é representada por dolomita branca, hematita e traços de willemita com valores mais elevados em $\mathrm{Mn}, \mathrm{Ba}, \mathrm{Cr}, \mathrm{Cd}, \mathrm{Ni}, \mathrm{Pb}$ e As, a qual corta o estágio principal de mineralização; (4) a fase mais tardia do estágio mineralizante é compreendida por Zn-clorita e quartzo, os quais foram precipitados pela mistura de fluidos de mais altas temperaturas e salinidades com fluidos de temperaturas e salinidades mais baixas $\left(\sim 170^{\circ}\right.$ a $190^{\circ} \mathrm{C} / 12,2$ a $18,4 \%$ em peso de $\mathrm{NaCl}$ equiv. e $\sim 90^{\circ}$ a $130^{\circ} \mathrm{C} / 5,8$ a $9,9 \%$ em peso de $\mathrm{NaCl}$ equiv., respectivamente). A composição dos fluidos de maiores temperaturas e salinidades é $\mathrm{H}_{2} \mathrm{O}-\mathrm{NaCl}$.

- A mineralização nos membros Morro do Pinheiro Superior e Pamplona Inferior exibem características similares, com valores mais elevados em $\mathrm{SiO}_{2}, \mathrm{Fe}_{2} \mathrm{O}_{3}, \mathrm{P}_{2} \mathrm{O}_{5}, \mathrm{Mn}$, As, Ge, $\mathrm{Hg}$, In, U, V, W, Ag, Cd, Cu, Mo, Ni, Pb, Sb, Se e Zn.; no entanto aquele apresenta maiores concentrações de $\mathrm{Zn}$ e $\mathrm{Cu}$, enquanto este exibe maiores concentrações de $\mathrm{Pb}$, Mn, As e V, além de apresentar Sb anômalo.

- A mineralização é caracterizada por composições isotópicas de $\mathrm{C}$ e $\mathrm{O}$ mais leves. Os valores isotópicos mais baixos de $\mathrm{O}$ refletem composições mais leves do fluido metalífero, mas também deve estar relacionada à mistura deste fluido com água meteórica evoluída. 
- Os estágios de alteração sulfetada incluem: (1) alteração de pirita representada por pirita, esfalerita, dolomita branca, hematita fina e quartzo tardio. Essa alteração apresenta valores mais elevados em $\mathrm{Na}_{2} \mathrm{O}, \mathrm{SiO}_{2}, \mathrm{Sr}, \mathrm{Ba}, \mathrm{Cu}, \mathrm{Ni}, \mathrm{As}, \mathrm{Re}$ e $\mathrm{S}$. A composição isotópica de dolomita é similar à composição de dolomita vermelha e branca do estágio principal de mineralização e da terceira fase do estágio mineralizante, respectivamente. Isso que sugere a mesma fonte de fluidos metalíferos para esses estilos de alteração hidrotermal. Quartzo tardio da alteração de pirita evidencia a mistura de fluidos dos mesmos fluidos envolvidos na precipitação de quartzo tardio do estágio mineralizante, o que indica uma relação genética entre essas duas fases minerais desses dois estilos de alteração hidrotermal; (2) alteração de galena é compreendida por esfalerita, galena, calcocita, greenockita, covelita e dolomita branca, a qual corta os corpos mineralizados com willemita e é interpretada como sendo tardia à alteração de pirita.

- Os locais com mineralizações ricas em sulfetos apresentam uma significante maior introdução de matéria orgânica ou hidrocarbonetos, os quais devem ter fornecido enxofre reduzido.

- Os dados isotópicos e de inclusões fluidas indicam que os fluidos associados as mineralizações de zinc silicatado e as occorrências sulfetadas são similares aquelas documentadas nos depósitos sulfetados (Morro Agudo, Ambrósia e Fagundes). Isto sugere que os fluidos associados as mineralizações de metais de base tiveram uma circulação ampla no Grupo Vazante.

- Os fluidos associados as fases mais precoses, interpretados como diagenéticos, tem composições e temperatures bem distintas dos fluidos mineralizantes. 


\section{Capítulo 4}

\section{REFERÊNCIAS}

AMARAL, G. 1968ª Geologia e depósitos de minério na região de Vazante, Estado de Minas Gerais. Tese de Doutorado, Escola Politécnica, Universidade de São Paulo, Brasil, 133 p.

AMARAL, G. 1968b. Contribuição ao conhecimento dos depósitos de Zn-Pb-Cu-Ag da Serra do Poço Verde, Vazante, Estado de Minas Gerais. Congresso Brasileiro de Geologia. Belo Horizonte,1969. Anais, 22, 13-31.

AMARAL, G., KAWASHITA, K. 1967. Determinação da idade do Grupo Bambuí pelo método Rb/Sr. Congresso Brasileiro de Geologia, SBG. Anais, 21, 214-217.

ALKIMIN, F.F., MARTINS-NETO, M.A. 2012. Proterozoic first-order sedimentary sequences of the São Francisco craton, eastern Brazil, Marine and Petroleum Geology, 33, 127-139.

ALMEIDA, F.F.M., 1993. Limites do cráton do São Francisco em Minas Gerais - Síntese dos conhecimentos. Simpósio do Cráton do São Francisco, SBG, Salvador. Anais, 256-259.

ALMEIDA, F.F.M. \& HASUI, Y. 1981. Brazilian structural provinces: An introduction. Earth-Science Reviews, 17, 1-29.

ALMEIDA, F.F.M. \& HASUI, Y. 1984. Introdução. In: Almeida, F F M de; Hasui, Y. (orgs.). O PréCambriano do Brasil. São Paulo: Editora Egard Blucher, 1-5.

ARAÚJO FILHO, J.O. 1999. Structural characteristics and tectonic evolution of the Pirineus sintaxis, Central Brazil. Tese de PhD, Univ. of Illinois, $418 \mathrm{p}$.

AZMY, K., KENDALL, B., CREASER, R.A., HEAMAN, L., de OLIVEIRA, T.F. 2008. Global correlation of the Vazante Group, Sao Francisco Basin, Brazil: Re-Os and U-Pb radiometric age constraints. Precambrian Research, 164, 160-172.

BABINSKY, M. MONTEIRO, L.V.S., FETTER, A.H., BETTENCOURT, J.S., OLIVEIRA, T.F. 2005. Isotope geochemistry of the mafic dikes from the Vazante Nonsulfide Zinc Deposit, Brazil. Journal of South American Earth Sciences, 18, 293-304.

BAIA, F. H. 2013. Brechas hidrotermais da mina do Cercado e das ocorrências Olho D’água, Mata II e Pamplona: implicações metalogenéticas e prospectivas para zinco na região de Vazante, MG. Dissertação de Mestrado, Universidade Estadual de Campinas, Instituto de Geociências, 198 p.

BASUKI, N.I., SPOONER, E.T.C. 2002. A Review of Fluid Inclusion Temperatures and Salinities in Mississippi Valley-type $\mathrm{Zn}-\mathrm{Pb}$ Deposits: Identifying Thresholds for Metal Transport. Explor. Mining Geol., 11(1-4), 1-17.

BETTENCOURT, J.S., MONTEIRO, L.V.S., BELlO, R.S., OlIVEIRA, T.F., JULIANI, C. 2001. Metalogênese do zinco e do chumbo na região de Vazante-Paracatu, Minas Gerais. In: Pinto, C.P., Martins-Neto, M.A. (eds.), Bacia do São Francisco, Geologia e Recursos Naturais. SBG-MG, Belo Horizonte, 159-196.

BEZ, L. 1980. Evolução mineralógica e geoquímica do depósito de zinco e chumbo de Morro Agudo, Paracatu, MG. Congresso Brasileiro de Geologia, Balneário Camburiú 3, SBG, 31, 1402-1416.

BODNAR, R.J. 1993. Revised equation and table for determining the freezing point depression of $\mathrm{H}_{2} \mathrm{O}-$ $\mathrm{NaCl}$ solutions. Geochimica et Cosmochimica Acta, 57, 683-684.

BORISENKO, A.S. 1978. Study of the salt composition of solutions of gas-liquid inclusions in minerals by the cryometric method. Sov,. Geol. Geophys., 18, $11-19$. 
BRITO NEVES, B.B., TEIXEIRA, W., TASSINARI, C.C.G., KAWASHITA, K. 1990. A contribution to the subdivision of the Precambrian South America. Revista Brasileira de Geociências, 20, 267-276.

BRITO NEVES, B.B., NETO, M.C.C., FUCK, R.A. 1999. From Rodinia to Western Gondwana: An approach to the Brasiliano-Pan African Cycle and orogenic collage. Episodes, China, Pequim, 22(3), $155-166$.

BRUGGER, J., McPHAIL, D.C., WALLACE, M., WATERS, J. 2003. Formation of willemite in hydrothermal environments. Economic Geology, 98, 819-835.

BURNS, S.J., BAKER, P.A., SHOWERS, W.J. 1988. The factors controlling the formation and chemistry of dolomite in organic-rich sediments, Miocene Drakes Bay Formation, California. In: Shukla, V., Baker, P.A. (eds.), Sedimentology and Geochemistry of Dolostones. Special Publication, Society of Economic Paleontologists and Mineralogists, 43, 41-52.

CAMPBELl, A.R., PANTER, K.S. 1990. Comparison of fluid inclusions in coexisting (cogenetic?) wolframite, cassiterite, and quartz from St. Michael's Mount and Cligga Head, Cornwall, England. Geochimica et Cosmochimica Acta, 54, 673-681.

CAMPOS NETO, M.C. 1984a. Geometria e fases de dobramentos brasilianos superpostos no oeste de Minas Gerais. Revista Brasileira de Geociências, 14, 60-68.

CAMPOS NETO, M.C. 1984b. Litoestratigrafia, relações estratigráficas e evolução paleogeográfica dos grupos Canastra e Paranoá (região Vazante-Lagamar, MG). Revista Brasileira de Geociências, 14, 8191.

CARVAlHO, P., GUIMARÃES, D., DEQUECH, D. 1962. Jazida plumbo-zincífera do Município de Vazante, Minas Gerais. Divisão de Fomento da Produção Mineral, DFPM, Boletim, 110, 147 p.

CASSEDANE, J., LASSERRE, M. 1969. Etude geologique et analyse isotopique, par la methode au plomb, de quelques galenes du Bresil. Bulletin du Bureau de Recherches Geologiques et Minieres (Section 2: Geologie des Gites Mineraux) 1, 71-83.

CLOUD, P. \& DARDENNE, M.A. 1997. Contribuição à sedimentologia e estratigrafia do Grupo Bambuí no norte de Minas Gerais. $9^{\circ}$ Simpósio, de Geologia de Minas Gerais, Ouro Preto, SBG, Anais, 14, 8182.

COSTA, I.A.M., ANGEIRAS, A.G. 1971. Geosynclinal evolution in the epi-Baikalian platform of Central Brazil. Geologische Rundchau, 60(2), 1024-1050.

CUNHA, I.A. 1999. Estudos de Inclusoes Fluidas e de isotopos de enxofre dos corpos de minério de Zn$\mathrm{Pb}$ de Morro Agudo, Minas Gerais. Dissertação de Mestrado, Universidade Federal da Bahia, 126p.

CUNHA, I. de A., COELHO, C.E.S. MISI, A. 2000. Fluid inclusion study of the Morro Agudo Pb-Zn deposit, Minas Gerais, Brazil. Revista Brasileira de Geociências, 30(2), 318 - 321.

CUNHA, I.A., BABINSKY, M., MISI, A. 2003. Pb isotopic constraints on the mineralization from the Vazante Group, Minas Gerais, Brazil. South American Symposium on Isotope Geology, Short Papers, 4, 727-730.

CUNHA, I.A., MISI, A., BABINSKY, M. 2001. Lead isotope signatures of galenas from Morro Agudo $\mathrm{Pb}-\mathrm{Zn}$ deposit, Minas Gerais, Brazil. In: Misi, A., Teixeira, J.B.G. (eds.), Proterozoic Base Metal Deposits of Africa and South America. Proceedings of the First IGCP 450 Field Workshop. CNPq/UNESCO/IUGS, Belo Horizonte and Paracatu (MG), Brazil, 45- 47.

DARDENNE, M.A. 1978. Geologia da região de Morro Agudo (MG). Núcleo Centro-Oeste, SBG, Boletim, 7-8, 68-94.

DARDENNE, M.A. 1978a. Geologia da região de Morro Agudo (MG). Sociedade Brasileira de Geologia, Núcleo Centro-Oeste, Boletim, 7/8, 68-94.

DARDENNE, M.A. 1978b. Síntese sobre a estratigrafia do Grupo Bambuí no Brasil Central. 30 Congresso Brasileiro de Geologia (Recife), Anais, 2, 597-610. 
DARDENNE, M.A. 1979. Les minéralisations de plomb, zinc, flúor du Protérozoïque Supérieur dans le Brésil Central. Tese de Doutorado, Université Paris VI, 251 p.

DARDENNE, M.A. 2000. The Brasília Fold Belt. In: Cordani, U.G., Milani, E.J., Thomaz Filho, A., Campos, D.A. (eds). The Tectonic Evolution of South America. $31^{\text {st }}$ International Geological Congress, Rio de Janeiro, $231-263$.

DARDENNE, M.A., CAMPOS, E.G., MARTINS, F.L., ALVARENGA, C.J.S. 1997. Geologia da região de Alto Paraíso de Goiás/Parque Nacional da Chapada dos Veadeiros, GO. FAPDF (geologia do Distrito Federal e Entorno Próximo), relatório interno não publicado.

DARDENNE, M.A., FARIA, A., ANDRADE, G.F. 1976. Occurrence de stromatolithes columnaires dans le Groupe Bambuí (Goiás, Brésil). Academia Brasileira de Geologia, Núcleo Centro-Oeste, Anais, 7/8, 85-97.

DARDENNE, M.A., FREITAS-SILVA, F.H. 1999. Pb-Zn ore deposits of Bambui and Vazante Groups, in the São Francisco Craton and Brasília Fold Belt, Brazil. In: Silva M. da G. da, Misi, A. (eds.), Base metal deposits of Brazil. Ministério de Minas e Energia/ Companhia de Pesquisa de Recursos Minerais Minerais/Departamento Nacional de Produção Mineral, 75-83.

DARDENNE, M.A., FREITAS-SILVA, F.H., SOUZA, J.C.F. de, CAMPOS, J.E.G. 1998. Evolução tectono-sedimentar do Grupo Vazante no contexto da Faixa de Dobramentos Brasília. Congresso Brasileiro de Geologia, 40, , Sociedade Brasileira de Geologia, Belo Horizonte, Anais, Resumos 26.

DARDENNE, M.A., SCHOBBENHAUS, C.S. 2001. Metalogênese do Brasil. Editora Universidade de Brasília/CNPq, Brasília. 392 p.

DAVIS, D.W., LOWENSTEIN, T.K., SPENCER, R.J. 1990. Melting behavior of fluid inclusions in laboratory-grown halite crystals in the systems $\mathrm{NaCl}-\mathrm{H}_{2} \mathrm{O}, \mathrm{NaCl}-\mathrm{KCl}-\mathrm{H}_{2} \mathrm{O}, \mathrm{NaCl}-\mathrm{MgCl}_{2}-\mathrm{H}_{2} \mathrm{O}$ and $\mathrm{NaCl}-\mathrm{CaCl}_{2}=\mathrm{H}_{2} \mathrm{O}$. Geochemica et Cosmochimica Acta, 54, $591-601$.

DELGADO, I.M. \& PEDREIRA, A.J. 1995. Tectono-Geological Map of Brazil, 1:7,000,000 scale. Geological Survey of Brazil - Companhia de Pesquisa de Recursos Minerais - CPRM, Rio de Janeiro.

DRESCH, R.A.C. 1987. Aspectos geoquímicos da Jazida de Morro Agudo, Paracatu, MG. Congresso Brasileiro de Geoquímica, Porto Alegre, 1, 5-27.

EVERETT, C.E., WILKINSON, JJ., BOYCE, A.J., ELLAM, R.M., GLEESON, S.A., FALLICK, A.E. 1999. The genesis of Irish-type $\mathrm{Zn}-\mathrm{Pb}$ deposits: Characterization and origin of the principal ore fluid. In: Stanley, C.J. (Ed.), Mineral Deposits: Processes to Processing. Proc. $5^{\text {th }}$ Biennial SGA Meeting and $10^{\text {th }}$ Quadrennial IAGOD Symposium, London A.A. Balkena, Rotterdam, 845-848.

FREITAS-SILVA, F.H., DARDENNE, M.A. 1997. Pb/Pb isotopic patterns of galenas from Morro do Ouro (Paracatu Formation), Morro Agudo/Vazante (Vazante Formation) and Bambuí Group deposits. South American Symposium on Isotope Geology, Extended Abstracts, 118- 120.

FONSECA, M.A. 1996. Estilos estruturais e arcabouço tectônico do segmento setentrional da Faixa Brasília. Tese de Doutorado, IG/UnB, Brasília, 172 p.

FONSECA, M.A. \& DARDENNE, M.A. 1995. Estilos estruturais e a compartimentação tectônica do segmento setentrional da Faixa Brasília. 5 Simp. Nac. Est. Tec. Gramado, SBG, Anais, 280-283.

FONSECA, M.A., DARDENNE, M.A., UHLEIN,, A. 1995. Faixa Brasília, Setor Setentrional: estilos estruturais e arcabouço tectônico. Revista Brasileira de Geociências, 25(4), 267-278.

FUCK, R.A. 1994. A Faixa Brasília e a compartimentação tectônica na Província Tocantins. 4º Simpósio de Geologia do Centro-Oeste, SBG, Brasília, Anais, 4, 184-187.

FUCK, R.A., JARDIM DE SÁ, E.F., PIMENTEL, M.M., DARDENNE, M.A., PEDROSA-SOARES, A.C. 1993. As faixas de dobramentos marginais do Cráton do São Francisco: síntese dos conhecimentos. In: Dominguez, J. M. L. \& Misi, A. (eds.), O Cráton do São Francisco, SBG/SGM/CNPq, Salvador, 165-181. 
FUCK, R.A., PIMENTEL, M.M., SILVA, J.H.D. 1994. Compartimentação tectônica na porção oriental da Província Tocantins. $38^{\circ}$ Congresso Brasileiro de Geologia, Camboriú, SBG, Anais, 1, 215-216.

GEBOY, N.J., KAUFMAN, A.J., WALKER, R.J., MISI, A., OLIVEIRA, T.F., MILLER, K.E., AZMY, K., KENDALL, B., POUTON, S.W. 2013. Re-Os age constraints and new observations of glacial deposits in the Mesoproterozoic Vazante Group Brazil. Precambrian Research. 238, 199-213.

GOLDSTEIN, R.H. \& REYNOLDS, T. J. 1994. Systematics of fluid inclusions in diagenetic minerals. Society for sedimentary geology, SEPM Short Course, v. 31, Tulsa, Oklahoma, 199 p.

HITZMAN, M.W. 1997. Sediment hosted $\mathrm{Zn}-\mathrm{Pb}$ and $\mathrm{Au}$ depostis in the Proterozoic Paracatu-Vazante Fold Belt, Minas Gerais, Brazil. Abstracts with programs, Geological Society of America 29, A408.

HITZMAN, M.W., REYNOLDS, N.A., SANGSTER, D.F., ALLEN, C.R., CARMAN, C.E., 2003. Classification, Genesis, and Exploration Guides for Nonsulfide Zinc Deposits. Economic Geology, 98, 685-714.

HITZMAN, M.W., THORMAN, C.H., ROMAGNA, G., OLIVEIRA, T.F. de, DARDENNE, M.A., DREW, L.J. 1995. The Morro Agudo Zn-Pb deposit, Minas Gerais, Brazil: A Proterozoic Irish-type carbonate hosted sedex replacement deposit. Abstracts with Programs, Geological Society of America 27, A408.

HORITA, J. 2014. Oxygen and carbon isotope fractionation in the system dolomite-water- $\mathrm{CO}_{2}$ to elevated temperatures. Geochimica et Cosmochimica Acta, 129: 111-124. In: Beaudoin, G., and Therrien, P., 2015, Stable Isotope Fractionation Factor Calculator, AlphaBeta. http://www2.ggl.ulaval.ca/cgi-bin/isotope/generisotope.cgi

IYER, S.S., HOEFS, J., KROUSE, H.R. 1992. Sulfur and lead isotope geochemistry of galenas from Bambuí Group, Minas Gerais, Brazil: implications on ore genesis. Economic Geology, 87, 437-443.

IYER, S.S., KROOUSE, H.P., BABINSKY, M., 1993. Isotope investigations on carbonate rocks hosted lead-zinc deposits from Bambuí Group, Minas Gerais, Brazil: implications for ore genesis and prospect evaluation. Simpósio do Cráton do São Francisco, Salvador, Anais, 2, 338- 339.

JONES, H.D. KESLER, S.E. 1992. Fluid inclusion gas geochemistry in east Tennesseee Mississippi Valley-type districts: evidence for fluid immiscibility and implications for depositional mechanisms. Geochimica et Cosmochimica Acta 56, 137-154.

LARGE, D. 2001, The geology of non-sulphide zinc deposits - An overview. Erzmetall, 54, 264-274.

LARGE, D.E. 1981. Sediment-hosted submarine exhalative lead-zinc deposits - a review of their geological characteristics and genesis. In: Wolf, K.H. (Ed.), Handbook of Strata-Bound and Stratiform Ore Deposits. Part III. Regional Studies and Specific, 9, 469-507.

MADALOSSO, A., 1980. Considerações sobre a paleogeografia do Grupo Bambuí na região de Paracatu, MG. $31^{\circ}$ Congresso Brasileiro de Geologia, Camburiú, SBG, Anais, 2, 772-785.

MADALOSSO, A. \& VALLE, C.R.O. 1978. Considerações sobre a estratigrafia e sedimentologia do Grupo Bambuí na região de Paracatu - Morro Agudo (MG). $30^{\circ}$ Congresso Brasileiro de Geologia, Recife, SBG, Anais, 2, 622-634.

MARINI, O.J., FUCK, R.A., DANNI, J.C.M., DARDENNE, M.A. 1981. A evolução geotectônica da Faixa Brasília e de seu embasamento. In: COM/SBG, Simpósio sobre o Cráton do São Francisco e suas Faixas Marginais, Salvador, Anais, 100-115.

MARINI, O.J., FUCK, R.A., DANNI, J.C.M., DARDENNE, M.A., LOGUÉRCIO, S.O., RAMALHO, R. 1984a. As faixas de dobramento Brasília, Uruaçu, Paraguai-Araguaia e o Maciço Mediano de Goiás. In: Schobbenhaus, C., Campos, D.A., Derze, G.R., Asmus, H.E. (coords.), Geologia do Brasil, Brasília, DNPM, p. 251-303. 
MARINI, O.J., FUCK, R.A., DARDENNE, M.A., DANNI, J.C.M. 1984b. Província Tocantins, Setores Central e Sudeste. In: Almeida, F. F. M. \& Hasui, Y. (eds.), O Pré-Cambriano do Brasil, São Paulo, Edgar Blücher, p. 205-264.

MISI, A., AZMY, K., KAUFMAN, A.J., OLIVEIRA, T.F., SANCHES, A.L., OLIVEIRA, G.D. 2014. Review of the geological and geochronological framework of the Vazante sequence, Minas Gerais, Brazil: Implication to metallogenic and phosphogenic models. Ore Geology Reviews, 63, 76-90.

MISI, A., IYER, S.S., KYLE, J.R., COELHO, C.E.S., FRANÇA-ROCHA, W.J.S., GOMES, A.S.R., CUNHA, I.A., CARVALHO, I.G. 1999. Geological and isotopic constraints on the metallogenic evolution of the Proterozoic sediment-hosted Pb-Zn (Ag) deposits of Brazil. Gondwana Research, 2, 47-65.

MISI, A., IYER, S.S., KYLE, J.R., COELHO, C.E.S., TASSINARI, C.C.G., FRANÇA-ROCHA, W.J.S., GOMES, A.S.R., CUNHA 1.A., TOULKERIDIS, T., SANCHES, A.L. 2000. A metallogenic evolution model for lead-zinc deposits of the Meso- and Neoproterozoic sedimentary basins of the São Francisco Craton, Bahia and Minas Gerais, Brazil. Revista Brasileira de Geociências, 30, 302-305.

MISI, A., IYER, S.S., TASSINARI, C.C.G. 1996. Boquira (2.5 Ga) and Morro Agudo (0,65 Ga) LeadZinc Deposits, Brazil: New SEDEX Subtypes? In: Cong. Bras.Geol., 39, IGCP Project 342: Age and Isotopes of South American Ores, Resumos extendidos, 7: 251-253.

MISI, A., TASSINARI, C.C.G., IYER, S.S. 1997. New isotope data from the Proterozoic lead-zinc (Ag) sediment-hosted sulfide deposits of Brazil: implications for their metallogenic evolution. SouthAmerican Symposium on Isotope Geology, Extended Abstract, 1, 201- 203.

MOERI, E. 1972. On a columnar stromatolite in the Precambrian Bambuí Group of Central Brazil. Eclog. Geol. Helv., 65(1), 185-195.

MONTEIRO, L.V.S. 1997. Contribuição à gênese das mineralizações de zinco da Mina de Vazante, MG. Dissertação de Mestrado, Instituto de Geociências, Universidade de São Paulo, 159 p.

MONTEIRO, L.V.S. 2002. Modelamento metalogenético dos depósitos de zinco de Vazante, Fagundes e Ambrósia, associados ao Grupo Vazante, Minas Gerais. Tese de doutorado, Instituto de Geociências, Universidade de São Paulo, 317 p.

MONTEIRO, L.V.S., BETTENCOURT, J.S. 2001. Genesis of the Vazante, Ambrósia and Fagundes Zn$(\mathrm{Pb})$ deposits (Minas Gerais, Brazil): Geologic and stable isotopic constraints. In: Misi, A., Teixeira, J.B. (eds.), Proterozoic base metal of Africa and South America. CNPq and UNESCO/IUGS, Belo Horizonte, 79-81.

MONTEIRO, LV.S., BETTTENCOURT, J.S., BELLO, R.M. da S., JULIANI, C., OLIVEIRA, T.F. 2003. Fluid inclusions and stable isotopic constraints on the genesis of the non-sulfide and sulfide zinc deposits in the Vazante-Paracatu Belt, Brazil. In: Cailteux, J.L.H. (Org.), Proterozoic Sedimenthosted Base Metal Deposits of Western Gondwana, UNESCO/IUGS, 163-167.

MONTEIRO, LV.S., BETTTENCOURT, J.S., GRAÇA, R. 1998a. Petrografia e quimismo mineral das mineralizações zincíferas de Vazante, MG. In: Congresso Brasileiro de Geologia, SBG, Belo Horizonte, Anais, 40157 p.

MONTEIRO, L.V.S., BETTENCOURT, J.S., JULIANI, C., OLIVEIRA, T.F. 2006. Geology, petrography, and mineral chemistry of the Vazante non-sulfide and Ambrósia and Fagundes sulfiderich carbonate-hosted Zn-(Pb) deposits. Minas Gerais, Brazil. Ore Geology Reviews, 28, 201-234.

MONTEIRO, L.V.S., BETTENCOURT, J.S., JULIANI, C., OLIVEIRA, T.F. 2007. Nonsulfide and sulfide-rich zinc mineralizations in the Vazante, Ambrósia and Fagundes deposits, MG, Brazil: mass balance and stable isotope constraints on the hydrothermal alterations. Gondwana Research, 11:362381

MONTEIRO, L.V.S., BETTENCOURT, J.S., OLIVEIRA, T.F. de, 2000. The Vazante, Ambrósia, and Fagundes (MG, Brazil). Neoproterozoic epigenetic zinc deposits: similarities, contrasting features and 
genetic implications. International Geological Congress, 31, Rio de Janeiro, Abstracts Volume (CD ROM).

MONTEIRO, L.V.S., BETTENCOURT, J.S., SPIRO, B. 1998b. Geometria, evolução fluidal e gênese da mineralização wilemítica da Mina de Vazante, MG. Evidências isotópicas. In: Congresso Brasileiro de GeologiaSBG, Belo Horizonte, Anais, 40. 142 p.

MONTEIRO, L.V.S., BETENCOURT, J.S., SPIRO, B., GRAÇA, R., OLIVEIRA, T.F. 1999. The Vazante zinc mine, MG, Brazil: constraints on fluid evolution and willemitic mineralization. Exploration and Mining Geology, 8, 21-42.

NOGUEIRA, G.S.M. 1993. Enquadramento litoestratigráfico, sedimentologia e evolução geoquímica do depósito fosfático de Lagamar, MG, Formação Vazante. Dissertação de Mestrado, IG/UnB, Brasília, $86 \mathrm{p}$.

OBERTHÜR, T., BLENKINSOP, T.G., HEIN, U.F., HÖPPER, M., HÖPPNER, M., HÖHNDORF, A., WEISER, T.W. 2000. Gold mineralization in the Mazowe área, Harare-Bindura-Shamva greenstone belt, Zimbabwe: II. Generic relationships deduced from mineralogical, fluid inclusion and stable isotope studies, and the Sm-Nd isotopic composition of scheelites. Mineralium Deposita, 53, 138 156.

OLIVEIRA, G.D., 2013. Reconstrução Paleoambiental e Quimioestratigráfica dos Carbonatos Hospedeiros do depósito de Zinco Silicatado de Vazante, MG. Dissertação de Mestrado $n^{\circ} 311$, IG/UnB, Brasília, 86 p.

OLIVO, G.R, PERCY, E., KYSER, K., OLIVEIRA, G., McGLADREY, A., SLEZAK, P., NETO, B.B., 2014. The Vazante and Northern Extension Hypogene Zinc Silicate Deposits: Evidence for Fluid Mixing During Mineralization. 21st General Meeting of the International Mineralogical Association, South Africa, September 1-5, 2014 (Talk and Abstract).

PERCY, E. 2014. Investigation of the isotopic signature of the Vazante and Northern Extension zinc silicate deposits, Minas Gerais, Brazil. Dissertação de Bacharelado em Ciências, Department of Geological Sciences and Engineering, Queen’s University, Kingston Ontário, Canadá.

PINHO, J.M.M. 1990. Evolução tectônica da mineralização de zinco de Vazante. Dissertação de Mestrado, Instituto de Geociências, Universidade de Brasília, 115 p.

PINHO, J.M.M., DARDENNE, M.A., RIGOBELLO, A.E. 1989. Evolução tectônica da mineralização de zinco de Vazante. In: Simpósio de Geologia no Núcleo Minas Gerais, 5. Anais, 10, 275-276.

PINHO, J.M.M., DARDENNE, M.A., RIGOBELLO, A.E. 1990. Caracterização da movimentação transcorrente da falha de Vazante, Vazante, MG. Congresso Brasileiro de Geologia, 36, SBG, Anais, 5, 2284- 2295 .

RIGOBELlO, A.E., BRANQUINHO, J.A., DANTAS, M.G.S., OLIVEIRA, T.F., NEVES FILHO, W. 1988. Mina de zinco de Vazante. In: Schobbenhaus, C. \& Coelho, C. E. S. (Eds.) Principais Depósitos Minerais do Brasil. DNPM, Brasília, 3, 101-110.

RODRIGUES, J.B., PIMENTEL, M.M., BUHN, B., MATTEINI, M., DARDENNE, M.A., ALVARENGA, C.J.S., ARMSTRONG, R.A. 2012. Provenance of the Vazante Group: New U-Pb, $\mathrm{Sm}-\mathrm{Nd}$. Lu-Hf isotopic data and implications for the tectonic evolution of the Neoproterozoic Brasilia Belt. Gondwana Research, 21, 439-450.

ROEDDER, E. 1984. Fluid inclusions. Mineralogical Society of America. Reviews in Mineralogy, vol. $12,644 \mathrm{p}$.

ROEDDER, E., BODNAR, R.J. 1997. Fluid inclusion studies of hydrothermal ore deposits. In: Barnes, H.L. (Ed)., Geochemistry of Hydrothermal Ore Deposits. Wiley, New York, 657-697.

ROMAGNA G., COSTA R.R. 1988. Jazida de zinco e chumbo de Morro Agudo, Paracatu, Minas Gerais. In: Schobbenhaus, C. \& Coelho, C.E.S. (Eds), Principais Depósitos Minerais do Brasil. 
Departamento Nacional da Produção Mineral / Companhia Vale do Rio Doce (DNPM / CRVD), 3, 111-121.

ROSCOE, J.S. \& ARAÚJO FILHO, J.O. 1994. Estudo estrutural preliminar do front de empurrão do Araxá sobre o Paranoá na porção oriental da megainflexão dos Pirineus, Goiás Central. Anais, $4^{\circ}$ Simp. Geol. Centro-Oeste, Brasília, SBG, 177-180.

RUBO, R.A., MONTEIRO, L.V.S. 2010. Sistemática de isótopos de oxigênio e carbono aplicada ao estudo da evolução metalogenética do depósito de Zn-Pb de Morro Agudo (MG). Revista Brasileira de Geociências, 40(3): 438-452.

SCHIMIDT, W. \& FLEISCHER, R. 1978. Estilo estrutural do pré-cambriano no sudoeste de Minas Gerais. Anais, $30^{\circ}$ Cong. Bras. Geol., Recife, SBG, 1, 431-434.

SCHOBBENHAUS, C., CAMPOS, D.A., DERZE, G.R., ASMUS, H. (Coords.) 1981. Geologia do Brasil, Brasília, DNPM, 501 p.

SEER, H.J. 1999. Evolução tectônica dos grupos Araxá, Ibiá e Canastra na sinforma Araxá, Minas Gerais. Tese de Doutorado, IG/UnB, Brasília, 267 p.

SEER, H.J., DARDENNE, M.A., FONSECA, M.A. 1998. Deformation and tectonic framework of the Meso/Neoproterozoic units in the southern Brasilia Fold Belt: implications on the geotectonic evolution. Abstracts, 14 $^{\circ}$ Int. Conf. On Basem. Tectonics, Ouro Preto, EM - DEGEO -UFOP, 57-61.

SHEPHERD, T.J., RANKIN, A.H., ALDERTON, D.H.M. 1985. A practical guide to fluid inclusion studies. Blackie and Son, 239 p.

SIMÕES, L.S.A. 1991. Dobras em bainhas macroscópicas relacionadas ao cisalhamento dúctil de baixo ângulo da nappe Araxá-Canastra no sudoeste de Minas Gerais. Anais, Simp. Nac. Est. Tec., Rio Claro, SBG, 11-13.

SIMÕES, L.S.A. 1995. Evolução tectono-metamórfica da nappe de Passos, sudoeste de Minas Gerais. Tese de Doutorado, IG/USP, São Paulo, 149 p.

SIMÕES, L.S.A. \& NAVARRO, G. 1996. Estruturação da Faixa Brasília na região de Araxá, MG. Anais, $39^{\circ}$ Cong. Bras. Geol., Salvador, SBG, 92-95.

SIMÕES, L.S.A. \& VALERIANO, C.M. 1990. Porção meridional da Faixa Brasília: estágio atual do conhecimento e problemas de correlação tectono-estratigráfica. Anais, $36^{\circ}$ Cong. Bras. Geol., Natal, SBG, 6, 2564-2575.

SINGER, D.A. 1995. World-class base and precious metal deposits: a quantitative analysis. Economic Geology, 90: 88-104.

SLEZAK, P. 2012. Geology, Minerology, and Geochemistry of the Vazante-Nothern Extension Zinc Silicate Deposit. Minas Gerais, Brazil. Dissertação de Mestrado, Queen's University, Kingston, Ontário, Canadá, 104 p.

SLEZAK, P.R., OLIVO, G.R., OLIVEIRA, G.D., DARDENNE, M.A. 2013. Geology, minerology and geochemistry of the Vazante Nothern Extension zinc silicate deposit, Minas Gerais, Brazil. Ore Geology Reviews, 56: 234-257.

SOUZA, J.C.F. 1997. Litoestratigrafia e sedimentologia da Formação Vazante na Região de Coromandel, MG. Dissertação de mestrado, IG/UnB, 75 p.

STACEY, J.S., KRAMERS, J.D. 1975. Approximation of terrestrial lead isotope evolution by a two-stage model. Earth and Planetary Science Letters, 26, 207- 221.

STRIEDER, A.J. 1993. Tectônica colisional no Brasil Central: evolução e implicações no padrão estrutural. Anais, $4^{\circ}$ Simp. Nac. Est. Tect., Belo Horizonte, SBG, 297-301.

STRIEDER, A.J. 1994. Deformação e metamorfismo na região de Santa Cruz de Goiás. Correlação tectono-estratigráfica e evolução tectônica regional. Tese de Doutorado, IG/UnB, Brasília, 258 p. 
TEIXEIRA, N.A. \& DANNI, J.C.M. 1978. Contribuição à estratigrafia do Grupo Araxá na região de Passos, MG. Anais, 30 Cong. Bras. Geol., Recife, SBG, 2: 700-710.

TOLCIN, A.C. 2011. Mineral Commodity Summaries 2009: Zinc. United States Geological Survey. Retrieved June 6, 2011.

VALERIANO, C.M. 1992. Evolução tectônica da extremidade meridional da Faixa Brasília, região da represa de Furnas, sudoeste de Minas Gerais. Tese de Doutorado, IG/USP, São Paulo, 198 p.

VALERIANO, C.M., PIMENTEL, M.M., HEILBRON, M., ALMEIDA, J.C.H., TROUW, R.A.J. 2008. Tectonic evolution of the Brasília Belt, Central Brazil, and early assembly of Gondwana. Geological Society, London, Special Publications 284: 197-210.

VIVIANI, C., ALMEIDA, D.R. ROMAGNA, G., LOPES, J.A., de SOUZA, J.C.F., de OLIVEIRA, T.F., BESSA, V. 2001. The Vazante and Morro Agudo Zn-Pb mines, Minas Gerais, Brazil. In: Misi, A., Teixeira, J.B. (eds.), Proterozoic Base Metal of Africa and South America. CNPq and UNESCO/IUGS, Belo Horizonte, 115-132.

WILKINSON, J.J. 2001. Fluid Inclusions in Hydrothermal Deposits. Lithos 55: 229-272.

WILKINSON, J.J. 2014. Sediment-hosted zinc-lead mineralization: processes and perspectives. Treatise on Geochemistry, 2nd Edition. http://dx.doi.org/10.1016/B978-0-08-095975-7.01109-8

WILKINSON, J.J., EVERETT, C.E., EYRE, S.L., BOYCE, A.J. 1998. Fluid flow and mineralization in the Irish Orefield. Programme with Abstracts, Mineral Deposits Studies Group AGM. University of St. Andrews, Scotland. 
Apêndices 
Apêndice 6. Análises de química mineral (em \% de peso) de dolomita do estágio precoce de alteração hidrotermal no Membro Pamplona Inferior.

\begin{tabular}{|c|c|c|c|c|c|c|c|c|c|c|c|c|c|c|c|c|c|c|c|c|}
\hline $\mathrm{Na}_{2} \mathrm{O}$ & MgO & MgO & $\mathrm{Al}_{2} \mathrm{O}_{3}$ & $\mathrm{~K}_{2} \mathrm{O}$ & $\mathrm{CaO}$ & $\mathrm{CaO}$ & $\mathrm{Cl}$ & $\mathrm{SiO}_{2}$ & $\mathrm{TiO}_{2}$ & $\mathrm{MnO}$ & $\mathrm{FeO}$ & $\mathrm{V}_{2} \mathrm{O}_{3}$ & Cd & $\mathrm{Zn}$ & $\mathbf{P b}$ & Sb & In & $(\mathrm{OH})$ & Total & Amostra \\
\hline 0,028 & 23,671 & 23,671 & 0,074 & 0,055 & 33,939 & 33,939 & 0,02 & 0,117 & 0 & 0,016 & 0,122 & 0,023 & 0,01 & 0 & 0 & 0 & & $\begin{array}{l}41,9 \\
41\end{array}$ & $\begin{array}{l}100,0 \\
01\end{array}$ & $267 \mathrm{~m}$ \\
\hline 0,006 & 24,342 & 24,342 & 0,004 & 0,009 & 34,642 & 34,642 & 0,054 & 0,038 & 0 & 0,036 & 0,079 & 0,022 & 0 & 0 & 0 & 0 & & $\begin{array}{l}40,7 \\
81\end{array}$ & $\begin{array}{l}100,0 \\
01 \\
\end{array}$ & $267 \mathrm{~m}$ \\
\hline 0,043 & 22,718 & 22,718 & 0,09 & 0,029 & 34,390 & 34,39 & 0,004 & 0,036 & 0 & 0,042 & 0,084 & 0,012 & 0,001 & 0,021 & 0 & 0,005 & & $\begin{array}{l}42,5 \\
53 \\
\end{array}$ & 100 & $267 \mathrm{~m}$ \\
\hline 0,039 & 23,206 & 23,206 & 0,484 & 0,117 & 33,968 & 33,968 & 0,041 & 0,504 & 0,061 & 0,039 & 0,185 & 0 & 0 & 0,011 & 0 & 0 & & $\begin{array}{l}41,3 \\
64\end{array}$ & $\begin{array}{l}99,99 \\
9\end{array}$ & $267 \mathrm{~m}$ \\
\hline 0,064 & 23,512 & 23,512 & 0,091 & 0,018 & 33,909 & 33,909 & 0,009 & 0,159 & 0,088 & 0,061 & 0,373 & 0 & 0 & 0 & 0 & 0 & & $\begin{array}{l}41,7 \\
19\end{array}$ & $\begin{array}{l}100,0 \\
01\end{array}$ & $267 \mathrm{~m}$ \\
\hline 0,043 & 22,461 & 22,461 & 0,079 & 0,036 & 34,007 & 34,007 & 0,017 & 0,124 & 0 & 0 & 0,218 & 0,03 & 0,01 & 0 & 0 & 0 & & $\begin{array}{l}42,9 \\
88 \\
\end{array}$ & $\begin{array}{l}99,99 \\
9 \\
\end{array}$ & $267 \mathrm{~m}$ \\
\hline 0 & 22,774 & 22,774 & 0,019 & 0,02 & 33,186 & 33,186 & 0,031 & 0 & 0,028 & 0,132 & 0,804 & 0 & 0 & 0 & 0 & 0 & & $\begin{array}{l}43,0 \\
12 \\
\end{array}$ & $\begin{array}{l}99,99 \\
9 \\
\end{array}$ & $267 \mathrm{~m}$ \\
\hline 0,043 & 24,088 & 24,088 & 0 & 0,008 & 34,737 & 34,737 & 0 & 0 & 0,094 & 0,199 & 0,552 & 0 & 0,004 & 0 & 0 & 0,004 & & $\begin{array}{l}40,2 \\
79 \\
\end{array}$ & 100 & $267 \mathrm{~m}$ \\
\hline 0 & 22,787 & 22,787 & 0 & 0 & 33,791 & 33,791 & 0 & 0,036 & 0,034 & 0,1 & 1473 & 0 & 0,021 & 0,443 & 0,191 & 0,029 & 0 & $\begin{array}{l}40,9 \\
98\end{array}$ & $\begin{array}{l}100,0 \\
01\end{array}$ & $127 \mathrm{~m}$ \\
\hline 0 & 22,559 & 22,559 & 0,02 & 0,014 & 33,832 & 33,832 & 0,005 & 0,055 & 0 & 0,173 & 0,57 & 0 & 0,001 & 0,267 & 0,007 & 0,052 & 0,003 & $\begin{array}{l}41,0 \\
85\end{array}$ & $\begin{array}{l}99,99 \\
9\end{array}$ & $127 \mathrm{~m}$ \\
\hline 0 & 22,668 & 22,668 & 0 & 0 & 34,055 & 34,055 & 0,009 & 0,043 & 0 & 0,16 & 1174 & 0 & 0 & 0,236 & 0,198 & 0,029 & 0 & $\begin{array}{l}41,2 \\
33 \\
\end{array}$ & $\begin{array}{l}100,0 \\
03 \\
\end{array}$ & $127 \mathrm{~m}$ \\
\hline 0 & 22,800 & 22,8 & 0,136 & 0 & 34,047 & 34,047 & 0,001 & 0,017 & 0 & 0,403 & 0,167 & 0 & 0,009 & 0,503 & 0,454 & 0,025 & 0 & $\begin{array}{l}40,9 \\
82 \\
\end{array}$ & $\begin{array}{l}100,0 \\
01 \\
\end{array}$ & $127 \mathrm{~m}$ \\
\hline 0 & 23,924 & 23,924 & 0,102 & 0 & 34,185 & 34,185 & 0,017 & 0,051 & 0 & 0 & 0,491 & 0 & 0,011 & 0,429 & 0,532 & 0,065 & 0,022 & $\begin{array}{l}40,4 \\
63 \\
\end{array}$ & $\begin{array}{l}99,99 \\
9 \\
\end{array}$ & $127 \mathrm{~m}$ \\
\hline
\end{tabular}


Apêndice 7. Análises de química mineral (em \% de peso) de dolomita do estágio principal de mineralização hidrotermal no Membro Pamplona Inferior.

\begin{tabular}{|c|c|c|c|c|c|c|c|c|c|c|c|c|c|c|c|c|c|c|c|c|}
\hline $\mathrm{Na}_{2} \mathrm{O}$ & MgO & MgO & $\mathrm{Al}_{2} \mathrm{O}_{3}$ & $\mathrm{~K}_{2} \mathrm{O}$ & $\mathrm{CaO}$ & $\mathrm{CaO}$ & $\mathrm{Cl}$ & $\mathrm{SiO}_{2}$ & $\mathrm{TiO}_{2}$ & $\mathrm{MnO}$ & $\mathrm{FeO}$ & $\mathrm{V}_{2} \mathrm{O}_{3}$ & $\mathrm{Cd}$ & $\mathrm{Zn}$ & $\mathrm{Pb}$ & Sb & In & $(\mathrm{OH})$ & Total & Amostra \\
\hline 0 & 21,149 & 21,149 & 0,003 & 0,01 & 33,937 & 33,937 & 0,007 & 0,013 & 0,022 & 0,114 & 0,898 & 0,047 & 0,054 & 1,363 & 0 & 0,008 & & 44,351 & 99,999 & $160 \mathrm{~m}$ \\
\hline 0 & 21,111 & 21,111 & 0 & 0,001 & 33,583 & 33,583 & 0,02 & 0,006 & 0 & 0,119 & 0,886 & 0 & 0,012 & 1,498 & 0 & 0 & & 42,905 & $\begin{array}{l}100,00 \\
1\end{array}$ & $160 \mathrm{~m}$ \\
\hline 0 & 21,195 & 21,195 & 0 & 0,021 & 33,473 & 33,473 & 0 & 0,08 & 0,146 & 0,092 & 0,917 & 0 & 0,032 & 1,461 & 0 & 0,01 & & 41,809 & 100 & $160 \mathrm{~m}$ \\
\hline 0,01 & 21,940 & 21,94 & 0 & 0 & 33,459 & 33,459 & 0,014 & 0,025 & 0,045 & 0,319 & 0,331 & 0 & 0,026 & 1,383 & 0 & 0 & & 43,568 & $\begin{array}{l}100,00 \\
3\end{array}$ & $160 \mathrm{~m}$ \\
\hline 0,037 & 21,295 & 21,295 & 0,023 & 0,017 & 33,801 & 33,801 & 0,008 & 0,081 & 0,056 & 0,121 & 0,863 & 0,002 & 0,001 & 1,428 & 0 & 0,001 & & 43,972 & 100 & $160 \mathrm{~m}$ \\
\hline 0 & 21,427 & 21,427 & 0 & 0 & 33,368 & 33,368 & 0,012 & 0,03 & 0 & 0,166 & 0,638 & 0,01 & 0,017 & 1,23 & 0 & 0 & & 44,317 & 100 & $160 \mathrm{~m}$ \\
\hline 0,019 & 22,587 & 22,587 & 0 & 0,012 & 33,886 & 33,886 & 0,003 & 0,006 & 0,034 & 0,279 & 0,271 & 0 & 0,055 & 1,135 & 0 & 0,005 & & 44,598 & 99,997 & $63 \mathrm{~m}$ \\
\hline 0,06 & 23,305 & 23,305 & 0,032 & 0 & 34,700 & 34,7 & 0,019 & 0 & 0,01 & 0 & 0,068 & 0,001 & 0,008 & 0,989 & 0 & 0 & & 44,902 & $\begin{array}{l}100,00 \\
1\end{array}$ & $63 \mathrm{~m}$ \\
\hline 0,08 & 21,799 & 21,799 & 0 & 0,002 & 33,555 & 33,555 & 0,018 & 0,031 & 0,107 & 0,211 & 0,636 & 0 & 0,026 & 1,131 & 0 & 0 & & 44,696 & 99,998 & $63 \mathrm{~m}$ \\
\hline 0,074 & 21,531 & 21,531 & 0,006 & 0,016 & 32,777 & 32,777 & 0,013 & 0,074 & 0,275 & 0,098 & 1,152 & 0,015 & 0,035 & 1,225 & 0 & 0,003 & & 40,122 & 99,998 & $172 \mathrm{~m}$ \\
\hline 0 & 21,500 & 21,5 & 0,076 & 0,004 & 32,460 & 32,46 & 0 & 0,297 & 0,028 & 0,181 & 1,137 & 0 & 0,051 & 1,233 & 0 & 0 & & 40,116 & 99,999 & $172 \mathrm{~m}$ \\
\hline 0,032 & 21,712 & 21,712 & 0,007 & 0 & 33,226 & 33,226 & 0 & 0,068 & 0,214 & 0,109 & 0,023 & 0,008 & 0,019 & 1,737 & 0 & 0 & & 40,493 & $\begin{array}{l}100,00 \\
3\end{array}$ & $172 \mathrm{~m}$ \\
\hline 0 & 21,208 & 21,208 & 0,018 & 0 & 33,113 & 33,113 & 0,002 & 0 & 0 & 0,627 & 0,108 & 0,023 & 0,064 & 1,723 & 0 & 0,001 & & 39,522 & 100 & $172 \mathrm{~m}$ \\
\hline 0,006 & 21,957 & 21,957 & 0,027 & 0 & 32,644 & 32,644 & 0,001 & 0,012 & 0 & 0,651 & 0,002 & 0,002 & 0,05 & 1,107 & 0 & 0,007 & & 39,555 & 99,997 & $172 \mathrm{~m}$ \\
\hline 0 & 23,932 & 23,932 & 0,04 & 0,026 & 33,893 & 33,893 & 0,018 & 0,009 & 0,22 & 0 & 0,948 & 0,047 & 0,022 & 0,359 & 0,047 & 0,031 & 0,008 & 40,146 & 99,998 & $172 \mathrm{~m}$ \\
\hline 0 & 24,388 & 24,388 & 0,028 & 0 & 33,397 & 33,397 & 0,011 & 0,023 & 0,056 & 0,135 & 0,75 & 0 & 0 & 0,517 & 0 & 0,027 & 0,008 & 41,01 & $\begin{array}{l}100,00 \\
1\end{array}$ & $172 \mathrm{~m}$ \\
\hline 0 & 23,648 & 23,648 & 0,065 & 0,005 & 33,317 & 33,317 & 0 & 0,019 & 0,242 & 0,106 & 0,876 & 0 & 0 & 0,471 & 0,03 & 0,028 & 0,005 & 40,58 & 99,998 & $172 \mathrm{~m}$ \\
\hline 0 & 24,265 & 24,265 & 0,133 & 0 & 34,233 & 34,233 & 0 & 0,051 & 0 & 0,03 & 0,031 & 0 & 0,022 & 0,647 & 0,343 & 0,037 & 0,001 & 41,049 & 99,999 & $172 \mathrm{~m}$ \\
\hline 0 & 20,828 & 20,828 & 0 & 0,009 & 33,053 & 33,053 & 0,034 & 0,037 & 0 & 0,214 & 0,111 & 0 & 0 & 3,368 & 0,295 & 0,033 & 0,009 & 40,882 & $\begin{array}{l}100,00 \\
1\end{array}$ & $172 \mathrm{~m}$ \\
\hline 0 & 21,362 & 21,362 & 0,009 & 0,019 & 32,937 & 32,937 & 0,032 & 0,036 & 0,235 & 0,587 & 0,2 & 0 & 0,02 & 2,324 & 0,938 & 0,022 & 0,008 & 39,476 & $\begin{array}{l}100,00 \\
1\end{array}$ & $172 \mathrm{~m}$ \\
\hline 0 & 23,200 & 23,2 & 0,059 & 0 & 33,802 & 33,802 & 0,014 & 0,02 & 0 & 0,008 & 0,068 & 0,034 & 0,236 & 0,787 & 0,49 & 0,042 & 0,006 & 38,695 & $\begin{array}{l}100,00 \\
1\end{array}$ & $172 \mathrm{~m}$ \\
\hline 0 & 24,289 & 24,289 & 0 & 0,003 & 33,909 & 33,909 & 0,034 & 0,057 & 0 & 0 & 0,029 & 0,037 & 0,065 & 0,444 & 0,662 & 0,042 & 0 & 39,797 & 100 & $172 \mathrm{~m}$ \\
\hline
\end{tabular}




\begin{tabular}{|c|c|c|c|c|c|c|c|c|c|c|c|c|c|c|c|c|c|c|c|c|}
\hline 0 & 24,120 & 24,12 & 0 & 0,005 & 33,991 & 33,991 & 0,006 & 0,031 & 0 & 0,006 & 0,775 & 0 & 0,019 & 0,036 & 0 & 0,025 & 0 & 40,842 & $\begin{array}{l}100,00 \\
2\end{array}$ & $127 \mathrm{~m}$ \\
\hline 0,039 & 24,226 & 24,226 & 0,095 & 0 & 33,583 & 33,583 & 0,01 & 0,031 & 0 & 0,036 & 0,836 & 0,028 & 0 & 0,124 & 0,042 & 0,037 & 0 & 41,089 & $\begin{array}{l}100,00 \\
2\end{array}$ & $127 \mathrm{~m}$ \\
\hline 0 & 21,611 & 21,611 & 0,086 & 0,017 & 33,823 & 33,823 & 0,015 & 0,016 & 0 & 0,079 & 0,006 & 0,058 & 0,018 & 2,576 & 0,111 & 0,032 & 0,002 & 40,74 & 99,998 & $127 \mathrm{~m}$ \\
\hline 0 & 21,883 & 21,883 & 0,125 & 0,059 & 33,904 & 33,904 & 0,01 & 0,491 & 0 & 0 & 0,193 & 0 & 0,008 & 2,544 & 0,201 & 0,023 & 0,004 & 40,734 & 100 & $127 \mathrm{~m}$ \\
\hline 0 & 21,488 & 21,488 & 0,295 & 0,021 & 32,733 & 32,733 & 0,014 & 0,348 & 0 & 0 & 0,191 & 0,015 & 0,008 & 1,832 & 0,742 & 0,037 & 0 & 41,231 & 100 & $127 \mathrm{~m}$ \\
\hline 0,007 & 23,859 & 23,859 & 0 & 0 & 34,855 & 34,855 & 0,027 & 0,007 & 0,074 & 0 & 0,165 & 0,065 & 0,002 & 0,064 & 0 & 0,036 & 0 & 41,051 & $\begin{array}{l}100,00 \\
1\end{array}$ & $127 \mathrm{~m}$ \\
\hline 0 & 23,493 & 23,493 & 0,04 & 0 & 34,175 & 34,175 & 0,006 & 0,001 & 0,012 & 0 & 0,884 & 0 & 0,016 & 0,042 & 0 & 0,072 & 0 & 41,434 & 99,999 & $127 \mathrm{~m}$ \\
\hline 0,001 & 23,778 & 23,778 & 0,233 & 0 & 34,191 & 34,191 & 0,004 & 0,002 & 0 & 0,103 & 0,765 & 0,051 & 0 & 0,003 & 0 & 0,031 & 0,007 & 40,793 & $\begin{array}{l}100,00 \\
1\end{array}$ & $127 \mathrm{~m}$ \\
\hline 0 & 23,296 & 23,296 & 0 & 0 & 34,565 & 34,565 & 0 & 0,057 & 0 & 0,068 & 0,141 & 0,018 & 0 & 0,112 & 0 & 0,054 & 0,015 & 40,466 & $\begin{array}{l}100,00 \\
1\end{array}$ & $127 \mathrm{~m}$ \\
\hline 0,011 & 23,242 & 23,242 & 0 & 0,014 & 34,252 & 34,252 & 0,001 & 0,021 & 0,006 & 0,083 & 0,904 & 0 & 0 & 0,032 & 0,026 & 0,061 & 0,013 & 41,198 & 99,999 & $127 \mathrm{~m}$ \\
\hline 0 & 22,951 & 22,951 & 0,14 & 0,004 & 34,116 & 34,116 & 0,017 & 0,152 & 0,016 & 0,253 & 0,18 & 0 & 0 & 0,477 & 0 & 0,033 & 0 & 41,189 & $\begin{array}{l}100,00 \\
1\end{array}$ & $127 \mathrm{~m}$ \\
\hline 0 & 23,228 & 23,228 & 0,081 & 0 & 34,782 & 34,782 & 0,019 & 0,002 & 0 & 0,06 & 0,356 & 0 & 0 & 0,118 & 0,013 & 0,004 & 0,025 & 44,351 & 99,999 & $127 \mathrm{~m}$ \\
\hline 0 & 22,900 & 22,9 & 0 & 0,039 & 34,293 & 34,293 & 0,022 & 0,117 & 0 & 0,182 & 0,119 & 0,068 & 0,013 & 0,849 & 0,056 & 0,077 & 0,005 & 42,905 & $\begin{array}{l}100,00 \\
1\end{array}$ & $127 \mathrm{~m}$ \\
\hline 0 & 23,010 & 23,01 & 0,112 & 0,032 & 34,326 & 34,326 & 0,025 & 0,09 & 0,038 & 0,066 & 0,113 & 0,028 & 0 & 0,561 & 0,125 & 0,056 & 0 & 41,809 & 100 & $127 \mathrm{~m}$ \\
\hline 0,002 & 23,794 & 23,794 & 0 & 0,017 & 34,235 & 34,235 & 0,023 & 0,024 & 0,101 & 0,105 & 0,253 & 0 & 0 & 0,112 & 0 & 0,011 & 0,01 & 43,568 & $\begin{array}{l}100,00 \\
3\end{array}$ & $127 \mathrm{~m}$ \\
\hline 0,025 & 23,679 & 23,679 & 0,033 & 0,015 & 34,676 & 34,676 & 0,014 & 0,033 & 0,046 & 0,042 & 0,22 & 0 & 0,005 & 0,018 & 0,053 & 0,048 & 0,001 & 43,972 & 100 & $127 \mathrm{~m}$ \\
\hline
\end{tabular}


Apêndice 8. Análises de química mineral (em \% de peso) de dolomita da alteração de pirita no Membro Morro do Pinheiro Superior.

\begin{tabular}{|c|c|c|c|c|c|c|c|c|c|c|c|c|c|c|c|c|c|c|c|c|}
\hline $\mathrm{Na}_{2} \mathrm{O}$ & $\mathrm{MgO}$ & MgO & $\mathrm{Al}_{2} \mathrm{O}_{3}$ & $\mathrm{~K}_{2} \mathrm{O}$ & $\mathrm{CaO}$ & $\mathrm{CaO}$ & $\mathrm{Cl}$ & $\mathrm{SiO}_{2}$ & $\mathrm{TiO}_{2}$ & $\mathrm{MnO}$ & $\mathrm{FeO}$ & $\mathrm{V}_{2} \mathrm{O}_{3}$ & $\mathrm{Cd}$ & $\mathrm{Zn}$ & $\mathrm{Pb}$ & $\mathrm{Sb}$ & In & $(\mathrm{OH})$ & Total & Amostra \\
\hline 0,001 & 23,010 & 23,01 & 0 & 0,012 & 35,085 & 35,085 & 0,011 & 0,063 & 0,022 & 0,102 & 1221 & 0 & 0,017 & 0 & 0 & 0,062 & 0,014 & 40,425 & 100 & $615 \mathrm{~m}$ \\
\hline 0 & 23,651 & 23,651 & 0 & 0,01 & 35,047 & 35,047 & 0 & 0,151 & 0 & 0,109 & 1089 & 0,031 & 0,001 & 0,002 & 0,037 & 0,028 & 0,012 & 39,594 & 99,998 & $615 \mathrm{~m}$ \\
\hline 0 & 23,372 & 23,372 & 0,079 & 0,024 & 33,851 & 33,851 & 0,02 & 0,018 & 0 & 0,234 & 1984 & 0,047 & 0 & 0,01 & 0,042 & 0,022 & 0 & 40,295 & 100,001 & $615 \mathrm{~m}$ \\
\hline 0,025 & 24,056 & 24,056 & 0 & 0 & 35,798 & 35,798 & 0,02 & 0,027 & 0 & 0,037 & 0,13 & 0,026 & 0,007 & 0,001 & 0 & 0,034 & 0 & 39,555 & 99,997 & $615 \mathrm{~m}$ \\
\hline $\begin{array}{l}(\mathrm{OH}) 0 \\
\end{array}$ & 23,987 & 23,987 & 0,099 & 0 & 34,193 & 34,193 & 0,009 & 0,044 & 0,102 & 0,051 & 0,387 & 0,003 & 0,002 & 0 & 0,002 & 0,018 & 0,003 & 40,795 & \begin{tabular}{|l|}
99,997 \\
\end{tabular} & $615 \mathrm{~m}$ \\
\hline 0,022 & 23,923 & 23,923 & 0,117 & 0,026 & 33,955 & 33,955 & 0,003 & 0,078 & 0 & 0,149 & 0,938 & 0,042 & 0 & 0 & 0,003 & 0,061 & 0,011 & 40,508 & 99,999 & $615 \mathrm{~m}$ \\
\hline 0,045 & 23,580 & 23,58 & 0,013 & 0,068 & 34,475 & 34,475 & 0 & 0,538 & 0,04 & 0,084 & 0,86 & 0,011 & 0,039 & 0,009 & 0,014 & 0,006 & 0,007 & 40,14 & 99,999 & $615 \mathrm{~m}$ \\
\hline 0,054 & 24,175 & 24,175 & 0,024 & 0 & 35,460 & 35,46 & 0,077 & 0,054 & 0 & 0 & 0,314 & 0 & 0 & 0,002 & 0 & 0,053 & 0,007 & 39,776 & 100,001 & $615 \mathrm{~m}$ \\
\hline 0,05 & 23,513 & 23,513 & 0 & 0,015 & 35,386 & 35,386 & 0,031 & 0,046 & 0,062 & 0,057 & 0,325 & 0 & 0 & 0,02 & 0 & 0,045 & 0 & 40,368 & 100,002 & $615 \mathrm{~m}$ \\
\hline 0,025 & 23,711 & 23,711 & 0 & 0,008 & 34,891 & 34,891 & 0,049 & 0,106 & 0,006 & 0,081 & 0,691 & 0 & 0,017 & 0,011 & 0,032 & 0,035 & 0 & 40,352 & 99,999 & $615 \mathrm{~m}$ \\
\hline 0,042 & 23,173 & 23,173 & 0,507 & 0,149 & 33,911 & 33,911 & 0,017 & 1,058 & 0 & 0,098 & 1376 & 0,006 & 0,012 & 0,015 & 0 & 0,026 & 0 & 39,532 & 100,003 & $615 \mathrm{~m}$ \\
\hline 0,044 & 23,448 & 23,448 & 0,306 & 0,147 & 34,376 & 34,376 & 0,018 & 0,825 & 0 & 0,158 & 0,686 & 0 & 0,026 & 0,015 & 0 & 0,041 & 0 & 39,924 & 100 & $615 \mathrm{~m}$ \\
\hline 0,027 & 23,169 & 23,169 & 0,293 & 0,161 & 34,802 & 34,802 & 0,018 & 1,173 & 0 & 0,042 & 0,585 & 0 & 0,011 & 0,003 & 0 & 0,051 & 0 & 39,509 & 99,998 & $615 \mathrm{~m}$ \\
\hline 0,011 & 23,011 & 23,011 & 0,281 & 0,012 & 33,893 & 33,893 & 0,02 & 0,031 & 0,034 & 0,276 & 1882 & 0 & 0,009 & 0,024 & 0 & 0,025 & 0 & 40,419 & 99,999 & $615 \mathrm{~m}$ \\
\hline 0,034 & 22,262 & 22,262 & 0,045 & 0 & 33,340 & 33,34 & 0 & 0 & 0 & 0,151 & 3211 & 0,03 & 0 & 0,009 & 0 & 0,018 & 0,001 & 40,636 & 99,999 & $615 \mathrm{~m}$ \\
\hline 0 & 23,282 & 23,282 & 0 & 0,012 & 33,681 & 33,681 & 0,021 & 0,027 & 0,006 & 0,117 & 2224 & 0 & 0 & 0 & 0 & 0,014 & 0,017 & 40,355 & \begin{tabular}{|l|}
99,999 \\
\end{tabular} & $615 \mathrm{~m}$ \\
\hline 0,016 & 23,185 & 23,185 & 0 & 0,006 & 33,377 & 33,377 & 0 & 0 & 0,022 & 0,135 & 2314 & 0,042 & 0,004 & 0 & 0 & 0,041 & 0,01 & 40,817 & 100,001 & $615 \mathrm{~m}$ \\
\hline 0,029 & 23,374 & 23,374 & 0,079 & 0,009 & 35,060 & 35,06 & 0,031 & 0,048 & 0,056 & 0,023 & 0,458 & 0,008 & 0,01 & 0,015 & 0 & 0,052 & 0 & 40,695 & 100,002 & $615 \mathrm{~m}$ \\
\hline 0,04 & 24,185 & 24,185 & 0,087 & 0 & 35,284 & 35,284 & 0,023 & 0,03 & 0,159 & 0 & 0,14 & 0,025 & 0,013 & 0,026 & 0 & 0,046 & 0 & 39,818 & 100,001 & $615 \mathrm{~m}$ \\
\hline 0,01 & 23,469 & 23,469 & 0,01 & 0,003 & 35,270 & 35,27 & 0,042 & 0 & 0 & 0,013 & 0,251 & 0,001 & 0,019 & 0 & 0,017 & 0,037 & 0,003 & 40,939 & 100 & $615 \mathrm{~m}$ \\
\hline 0,016 & 23,589 & 23,589 & 0 & 0,016 & 35,125 & 35,125 & 0,051 & 0,045 & 0,151 & 0,03 & 0,556 & 0,02 & 0,007 & 0,015 & 0 & 0,031 & 0 & 40,271 & 99,998 & $615 \mathrm{~m}$ \\
\hline 0,017 & 23,371 & 23,371 & 0 & 0,002 & 35,651 & 35,651 & 0,018 & 0,159 & 0,219 & 0 & 0,361 & 0 & 0 & 0 & 0 & 0,041 & 0,004 & 40,157 & 100,001 & $615 \mathrm{~m}$ \\
\hline 0,043 & 23,020 & 23,02 & 0,203 & 0,026 & 35,048 & 35,048 & 0,022 & 0,186 & 0 & 0 & 0,603 & 0,013 & 0,019 & 0 & 0,036 & 0,033 & 0,005 & 40,574 & 100,002 & $615 \mathrm{~m}$ \\
\hline 0,021 & 22,439 & 22,439 & 0,806 & 0,291 & 33,501 & 33,501 & 0,027 & 1,833 & 0,016 & 0,081 & 0,79 & 0 & 0,003 & 0,002 & 0 & 0,018 & 0,004 & 39,89 & 100,001 & $615 \mathrm{~m}$ \\
\hline 0,001 & 23,039 & 23,039 & 0 & 0,026 & 34,062 & 34,062 & 0 & 0,03 & 0 & 0,132 & 2271 & 0 & 0,004 & 0,038 & 0 & 0,049 & 0,004 & 40,161 & \begin{tabular}{|l|}
99,999 \\
\end{tabular} & $615 \mathrm{~m}$ \\
\hline
\end{tabular}




\begin{tabular}{|c|c|c|c|c|c|c|c|c|c|c|c|c|c|c|c|c|c|c|c|c|}
\hline 0,029 & 23,026 & 23,026 & 0,185 & 0 & 33,890 & 33,89 & 0 & 0,003 & 0,04 & 0,104 & 2,07 & 0,048 & 0 & 0,031 & 0,029 & 0,021 & 0 & 40,456 & 99,998 & $615 \mathrm{~m}$ \\
\hline 0,006 & 22,319 & 22,319 & 0,1 & 0,015 & 34,167 & 34,167 & 0,016 & 0,066 & 0 & 0,112 & 1771 & 0,011 & 0 & 0,091 & 0,023 & 0,064 & 0 & 41,25 & 100 & $615 \mathrm{~m}$ \\
\hline 0 & 22,731 & 22,731 & 0,14 & 0 & 33,968 & 33,968 & 0,026 & 0,03 & 0 & 0,292 & 2454 & 0 & 0 & 0,34 & 0,003 & 0,033 & 0,005 & 39,672 & 100,002 & $615 \mathrm{~m}$ \\
\hline 0 & 22,702 & 22,702 & 0,078 & 0,003 & 33,831 & 33,831 & 0,008 & 0,067 & 0 & 0,224 & 2572 & 0,015 & 0,004 & 0,438 & 0 & 0,05 & 0 & 39,5 & 100 & $615 \mathrm{~m}$ \\
\hline 0 & 22,454 & 22,454 & 0,141 & 0 & 33,838 & 33,838 & 0,003 & 0,065 & 0,01 & 0,289 & 2368 & 0 & 0,015 & 7,622 & 0 & 0,035 & 0,005 & 39,923 & 99,998 & $615 \mathrm{~m}$ \\
\hline 0 & 23,195 & 23,195 & 0,103 & 0,01 & 33,359 & 33,359 & 0,004 & 0,009 & 0 & 0,076 & 2446 & 0,024 & 0,039 & 0,2 & 0 & 0,05 & 0,006 & 39,995 & 99,998 & $615 \mathrm{~m}$ \\
\hline 0,023 & 23,090 & 23,09 & 0 & 0,01 & 33,623 & 33,623 & 0 & 0,013 & 0 & 0,088 & 2278 & 0,029 & 0 & 0,214 & 0 & 0,038 & 0,004 & 40,507 & 100 & $615 \mathrm{~m}$ \\
\hline 0 & 23,132 & 23,132 & 0 & 0,024 & 33,170 & 33,17 & 0 & 0,06 & 0 & 0,051 & 2237 & 0 & 0 & 0,626 & 0,006 & 0,068 & 0,008 & 39,828 & 100 & $615 \mathrm{~m}$ \\
\hline 0 & 22,773 & 22,773 & 0 & 0,003 & 34,011 & 34,011 & 0 & 0,048 & 0,073 & 0,103 & 2165 & 0 & 0,018 & 0,376 & 0 & 0,05 & 0,009 & 40,138 & 99,999 & $615 \mathrm{~m}$ \\
\hline 0 & 23,307 & 23,307 & 0,062 & 0 & 34,723 & 34,723 & 0,03 & 0,057 & 0 & 0,371 & 2204 & 0 & 0,047 & 5,156 & 0,124 & 0,013 & 0 & 38,4 & 99,999 & $615 \mathrm{~m}$ \\
\hline 0,05 & 23,320 & 23,32 & 0,074 & 0,018 & 35,329 & 35,329 & 0,091 & 0,021 & 0,248 & 0,068 & 0,765 & 0 & 0,015 & 0,017 & 0 & 0,046 & 0 & 39,773 & 100 & $615 \mathrm{~m}$ \\
\hline 0,029 & 23,365 & 23,365 & 0 & 0,024 & 34,877 & 34,877 & 0,032 & 0,018 & 0,135 & 0,009 & 0,962 & 0,03 & 0 & 0 & 0,05 & 0,071 & 0,001 & 40,484 & 99,999 & $615 \mathrm{~m}$ \\
\hline 0,016 & 23,414 & 23,414 & 0 & 0,007 & 34,601 & 34,601 & 0,016 & 0,175 & 0 & 0,148 & 0,987 & 0,076 & 0 & 0,002 & 0,005 & 0,032 & 0,002 & 40,402 & 99,997 & $615 \mathrm{~m}$ \\
\hline 0,039 & 23,863 & 23,863 & 0,026 & 0,002 & 35,285 & 35,285 & 0,017 & 0,043 & 0,248 & 0,107 & 0,52 & 0 & 0 & 0,005 & 0,014 & 0,036 & 0 & 39,593 & 99,999 & $615 \mathrm{~m}$ \\
\hline 0,048 & 23,594 & 23,594 & 0 & 0 & 35,175 & 35,175 & 0,029 & 0,02 & 0,074 & 0,076 & 0,085 & 0 & 0,013 & 0,014 & 0 & 0,043 & 0,011 & 40,875 & 99,999 & $615 \mathrm{~m}$ \\
\hline 0 & 23,608 & 23,608 & 0,08 & 0,02 & 33,587 & 33,587 & 0,016 & 0,028 & 0,01 & 0,213 & 2,3 & 0,025 & 0 & 0 & 0 & 0,016 & 0,003 & 39,886 & 100 & $615 \mathrm{~m}$ \\
\hline 0,024 & 22,712 & 22,712 & 0 & 0 & 33,829 & 33,829 & 0,019 & 0,025 & 0 & 0,009 & 2242 & 0,035 & 0,001 & 0 & 0 & 0,06 & 0 & 40,821 & 99,997 & $615 \mathrm{~m}$ \\
\hline 0,008 & 22,561 & 22,561 & 0 & 0,007 & 34,386 & 34,386 & 0,022 & 0,032 & 0 & 0,074 & 2241 & 0 & 0 & 0,013 & 0 & 0 & 0,012 & 40,581 & 99,999 & $615 \mathrm{~m}$ \\
\hline 0,02 & 23,340 & 23,34 & 0,05 & 0,016 & 33,534 & 33,534 & 0,027 & 0,041 & 0,297 & 0,117 & 2227 & 0,018 & 0 & 0,005 & 0,029 & 0,035 & 0,002 & 40,086 & 100,001 & $615 \mathrm{~m}$ \\
\hline 0,043 & 23,016 & 23,016 & 0 & 0,014 & 33,673 & 33,673 & 0,032 & 0,029 & 0,174 & 0,145 & 1,98 & 0,026 & 0,023 & 0,026 & 0,015 & 0,035 & 0,017 & 40,599 & 100,001 & $615 \mathrm{~m}$ \\
\hline
\end{tabular}


Apêndice 9. Análises de química mineral (em \% de peso) de dolomita da alteração de pirita no Membro Pamplona Inferior, próximo ao halo de alteração da alteração do estágio mineralizante.

\begin{tabular}{|c|c|c|c|c|c|c|c|c|c|c|c|c|c|c|c|c|c|c|c|c|}
\hline $\mathrm{Na}_{2} \mathrm{O}$ & MgO & $\mathrm{MgO}$ & $\mathrm{Al}_{2} \mathrm{O}_{3}$ & $\mathrm{~K}_{2} \mathrm{O}$ & $\mathrm{CaO}$ & $\mathrm{CaO}$ & $\mathrm{Cl}$ & $\mathrm{SiO}_{2}$ & $\mathrm{TiO}_{2}$ & $\mathrm{MnO}$ & $\mathrm{FeO}$ & $\mathrm{V}_{2} \mathrm{O}_{3}$ & $\mathrm{Cd}$ & $\mathrm{Zn}$ & $\mathbf{P b}$ & Sb & In & $(\mathrm{OH})$ & Total & Amostra \\
\hline 0 & 23,801 & 23,801 & 0 & 0 & 34,033 & 34,033 & 0,014 & 0,006 & 0 & 0,047 & 0,416 & 0 & 0 & 0 & 0 & 0 & & 41.685 & 99.999 & $267 \mathrm{~m}$ \\
\hline 0,002 & 23,663 & 23,663 & 0,032 & 0 & 33,970 & 33,97 & 0,019 & 0,031 & 0,057 & 0,122 & 0,479 & 0 & 0 & 0,005 & 0 & 0,005 & & 41.628 & 99.999 & $267 \mathrm{~m}$ \\
\hline 0,007 & 23,944 & 23,944 & 0,007 & 0,01 & 33,984 & 33,984 & 0,021 & 0 & 0,1 & 0,038 & 0,267 & 0,017 & 0 & 0 & 0 & 0 & & 41,61 & 100 & $267 \mathrm{~m}$ \\
\hline 0 & 23,905 & 23,905 & 0 & 0,001 & 33,867 & 33,867 & 0 & 0,048 & 0 & 0,037 & 0,522 & 0 & 0 & 0 & 0 & 0 & & 41.619 & 99.999 & $267 \mathrm{~m}$ \\
\hline 0,045 & 23,600 & 23,6 & 0,023 & 0,006 & 34,218 & 34,218 & 0,012 & 0 & 0 & 0,084 & 0,571 & 0,016 & 0,004 & 0,005 & 0 & 0,008 & & 41.429 & 100.001 & $267 \mathrm{~m}$ \\
\hline 0,008 & 23,434 & 23,434 & 0,003 & 0 & 34,195 & 34,195 & 0,002 & 0,031 & 0,1 & 0,023 & 0,468 & 0,011 & 0,008 & 0,015 & 0 & 0 & & 41.724 & 99.999 & $267 \mathrm{~m}$ \\
\hline 0 & 22,560 & 22,56 & 0,029 & 0,002 & 33,886 & 33,886 & 0,002 & 0,043 & 0,256 & 0,184 & 0,424 & 0 & 0 & 0,002 & 0 & 0 & & 42.615 & 100.001 & $267 \mathrm{~m}$ \\
\hline 0,026 & 23,565 & 23,565 & 0,01 & 0,016 & 34,334 & 34,334 & 0,015 & 0,018 & 0,094 & 0,13 & 0,386 & 0 & 0,025 & 0 & 0 & 0,001 & & 41,41 & 100.001 & $267 \mathrm{~m}$ \\
\hline 0 & 23,398 & 23,398 & 0,04 & 0 & 33,814 & 33,814 & 0 & 0,154 & 0,061 & 0,121 & 0,6 & 0 & 0 & 0,013 & 0 & 0,003 & & 41.811 & 99.999 & $267 \mathrm{~m}$ \\
\hline 0,033 & 23,111 & 23,111 & 0 & 0,018 & 33,428 & 33,428 & 0 & 0,05 & 0,104 & 0,124 & 0,971 & 0,038 & 0,008 & 0 & 0 & 0 & & 42.121 & 99.998 & $267 \mathrm{~m}$ \\
\hline 0,028 & 23,549 & 23,549 & 0,018 & 0,001 & 33,664 & 33,664 & 0,014 & 0,038 & 0 & 0,083 & 0,83 & 0,004 & 0,006 & 0,018 & 0 & 0,003 & & 41.774 & 100 & $267 \mathrm{~m}$ \\
\hline 0,011 & 23,687 & 23,687 & 0,001 & 0,016 & 33,711 & 33,711 & 0,002 & 0,063 & 0 & 0,15 & 0,301 & 0 & 0,007 & 0,06 & 0 & 0 & & 42.058 & 100 & $267 \mathrm{~m}$ \\
\hline 0,035 & 22,787 & 22,787 & 0,044 & 0 & 34,093 & 34,093 & 0,03 & 0,13 & 0,116 & 0,104 & 0,12 & 0,022 & 0,011 & 0,088 & 0 & 0,009 & & 42.525 & 99.999 & $267 \mathrm{~m}$ \\
\hline
\end{tabular}


Apêndice 10. Análises de química mineral (em \% de peso) de dolomita da alteração de galena no Membro Pamplona Inferior.

\begin{tabular}{|c|c|c|c|c|c|c|c|c|c|c|c|c|c|c|c|c|c|c|c|c|}
\hline $\mathrm{Na}_{2} \mathrm{O}$ & MgO & MgO & $\mathrm{Al}_{2} \mathrm{O}_{3}$ & $\mathrm{~K}_{2} \mathrm{O}$ & $\mathrm{CaO}$ & $\mathrm{CaO}$ & $\mathrm{Cl}$ & $\mathrm{SiO}_{2}$ & $\mathrm{TiO}_{2}$ & $\mathrm{MnO}$ & $\mathrm{FeO}$ & $\mathrm{V}_{2} \mathrm{O}_{3}$ & $\mathrm{Cd}$ & $\mathrm{Zn}$ & $\mathrm{Pb}$ & $\mathrm{Sb}$ & In & $(\mathrm{OH})$ & Total & Amostra \\
\hline 0,088 & 24,009 & 24,009 & 0,031 & 0,016 & 37,508 & 37,508 & 0,012 & 0,069 & 0 & 0,235 & 2,134 & 0,001 & 0 & 0,047 & 0,065 & 0,048 & 0,007 & 35,617 & 100 & $411 \mathrm{~m}$ \\
\hline 0,046 & 23,552 & 23,552 & 0 & 0 & 37,791 & 37,791 & 0,038 & 0,013 & 0,033 & 0,137 & 2432 & 0,018 & 0 & 0,01 & 0 & 0,045 & 0 & 35,57 & 99,998 & $411 \mathrm{~m}$ \\
\hline 0,019 & 23,684 & 23,684 & 0 & 0,025 & 37,584 & 37,584 & 0,03 & 0,006 & 0 & 0,149 & 2295 & 0,037 & 0 & 0,001 & 0,005 & 0,038 & 0,007 & 35,797 & 99,999 & $411 \mathrm{~m}$ \\
\hline 0,079 & 24,070 & 24,07 & 0,085 & 0 & 38,839 & 38,839 & 0,017 & 0,008 & 0,098 & 0,229 & 2149 & 0,027 & 0 & 0,014 & 0 & 0,011 & 0 & 34,165 & 99,999 & $411 \mathrm{~m}$ \\
\hline 0,039 & 23,832 & 23,832 & 0,011 & 0,006 & 37,830 & 37,83 & 0,017 & 0,002 & 0,049 & 0,315 & 2616 & 0,012 & 0 & 0,018 & 0,066 & 0,082 & 0,016 & 35,066 & 100 & $411 \mathrm{~m}$ \\
\hline 0,024 & 23,213 & 23,213 & 0,038 & 0,004 & 37,571 & 37,571 & 0 & 0,01 & 0,033 & 0,124 & 3379 & 0,033 & 0,014 & 0,016 & 0,057 & 0,046 & 0,007 & 35,407 & 99,999 & $411 \mathrm{~m}$ \\
\hline 0,01 & 24,202 & 24,202 & 0,027 & 0,012 & 37,983 & 37,983 & 0,007 & 0,016 & 0 & 0,156 & 2315 & 0,019 & 0 & 0,035 & 0 & 0,04 & 0,014 & 35,179 & 100 & $411 \mathrm{~m}$ \\
\hline 0,044 & 23,394 & 23,394 & 0,007 & 0,014 & 37,248 & 37,248 & 0 & 0,022 & 0 & 0,005 & 2,97 & 0,017 & 0,015 & 0,006 & 0,102 & 0,066 & 0,013 & 36,223 & 100,001 & $411 \mathrm{~m}$ \\
\hline 0,013 & 23,380 & 23,38 & 0 & 0,001 & 37,708 & 37,708 & 0 & 0,045 & 0,055 & 0,166 & 2,8 & 0 & 0 & 0,029 & 0 & 0,052 & 0 & 35,615 & 100,001 & $411 \mathrm{~m}$ \\
\hline 0,038 & 23,881 & 23,881 & 0 & 0,003 & 37,041 & 37,041 & 0,043 & 0,034 & 0 & 0,115 & 2264 & 0 & 0,008 & 0,012 & 0 & 0,028 & 0,006 & 36,446 & 99,999 & $411 \mathrm{~m}$ \\
\hline 0,03 & 23,946 & 23,946 & 0 & 0 & 37,458 & 37,458 & 0,001 & 0,048 & 0,033 & 0,225 & 2237 & 0,034 & 0 & 0,003 & 0,03 & 0,028 & 0 & 35,768 & 100,002 & $411 \mathrm{~m}$ \\
\hline 0,03 & 23,520 & 23,52 & 0,052 & 0,018 & 37,490 & 37,49 & 0,064 & 0 & 0 & 0,14 & 2166 & 0 & 0 & 0,004 & 0 & 0,057 & 0,012 & 36,429 & 99,999 & $411 \mathrm{~m}$ \\
\hline 0,03 & 22,883 & 22,883 & 0,054 & 0 & 37,434 & 37,434 & 0,039 & 0,027 & 0,234 & 0,071 & 2241 & 0 & 0,003 & 0,006 & 0 & 0,029 & 0 & 36,834 & 100,001 & $411 \mathrm{~m}$ \\
\hline 0,041 & 23,091 & 23,091 & 0,018 & 0,037 & 37,411 & 37,411 & 0 & 0,031 & 0 & 0,327 & 2766 & 0 & 0,02 & 0 & 0,011 & 0,018 & 0,014 & 35,979 & 100,002 & $411 \mathrm{~m}$ \\
\hline 0 & 22,814 & 22,814 & 0 & 0 & 36,780 & 36,78 & 0,019 & 0,007 & 0,127 & 0,209 & 1928 & 0,022 & 0,033 & 0,45 & 0 & 0,055 & 0 & 36,511 & 100,003 & $411 \mathrm{~m}$ \\
\hline 0,014 & 22,694 & 22,694 & 0,009 & 0,006 & 35,354 & 35,354 & 0,011 & 0,038 & 0 & 0,108 & 2254 & 0,016 & 0 & 0,109 & 0 & 0,048 & 0,005 & 38,993 & 100 & $411 \mathrm{~m}$ \\
\hline 0,018 & 24,447 & 24,447 & 0,052 & 0,003 & 37,530 & 37,53 & 0,047 & 0,016 & 0 & 0 & 1986 & 0 & 0 & 0,089 & 0,014 & 0 & 0,011 & 35,483 & 100,001 & $411 \mathrm{~m}$ \\
\hline 0,043 & 24,307 & 24,307 & 0 & 0 & 36,824 & 36,824 & 0,02 & 0,033 & 0,105 & 0,279 & 2775 & 0 & 0,02 & 0,095 & 0,078 & 0,032 & 0 & 35,506 & 100 & $411 \mathrm{~m}$ \\
\hline 0 & 24,476 & 24,476 & 0,023 & 0,004 & 37,634 & 37,634 & 0 & 0,024 & 0,099 & 0,202 & 2,65 & 0,035 & 0 & 0,042 & 0 & 0,022 & 0,008 & 34,495 & 99,998 & $411 \mathrm{~m}$ \\
\hline 0,011 & 23,714 & 23,714 & 0 & 0,003 & 37,301 & 37,301 & 0,012 & 0,009 & 0 & 0,226 & 2088 & 0 & 0 & 0,096 & 0 & 0,031 & 0 & 36,218 & 100 & $411 \mathrm{~m}$ \\
\hline 0 & 22,597 & 22,597 & 0 & 0,022 & 35,385 & 35,385 & 0,023 & 0,035 & 0 & 0,257 & 2713 & 0,021 & 0,003 & 0,235 & 0 & 0,038 & 0,01 & 38,405 & 100,002 & $411 \mathrm{~m}$ \\
\hline 0,017 & 22,866 & 22,866 & 0 & 0 & 37,896 & 37,896 & 0,002 & 0,01 & 0 & 0,184 & 3726 & 0,011 & 0,006 & 0,054 & 0,001 & 0,035 & 0 & 35,091 & 99,999 & $411 \mathrm{~m}$ \\
\hline 0,007 & 24,237 & 24,237 & 0 & 0,002 & 37,254 & 37,254 & 0,014 & 0,03 & 0 & 0,205 & 2201 & 0,061 & 0 & 0,031 & 0 & 0,028 & 0 & 35,524 & 99,999 & $411 \mathrm{~m}$ \\
\hline 0,004 & 24,142 & 24,142 & 0,066 & 0,014 & 37,566 & 37,566 & 0,049 & 0,003 & 0 & 0,215 & 2217 & 0,003 & 0 & 0,055 & 0,046 & 0,03 & 0,015 & 35,453 & 100,001 & $411 \mathrm{~m}$ \\
\hline 0,062 & 23,689 & 23,689 & 0 & 0 & 37,048 & 37,048 & 0,015 & 0,008 & 0 & 0,109 & 2556 & 0,024 & 0,032 & 0,018 & 0 & 0,023 & 0,008 & 36,179 & 99,999 & $411 \mathrm{~m}$ \\
\hline
\end{tabular}


Apêndice 11. Análises de química mineral (em \% de peso) de willemita da segunda fase do estágio mineralizante (estágio principal de mineralização) no Membro Pamplona Inferior.

\begin{tabular}{|c|c|c|c|c|c|c|c|c|c|c|c|c|c|c|c|c|c|c|c|c|c|c|}
\hline $\mathrm{Na}_{2} \mathrm{O}$ & MgO & $\mathrm{Al}_{2} \mathrm{O}_{3}$ & $\mathrm{~K}_{2} \mathrm{O}$ & $\mathrm{CaO}$ & $\mathrm{Cl}$ & $\mathrm{SiO}_{2}$ & $\mathrm{TiO}_{2}$ & $\mathrm{MnO}$ & $\mathrm{FeO}$ & $\mathrm{V}_{2} \mathrm{O}_{3}$ & $\mathrm{P}_{2} \mathrm{O}_{5}$ & $\mathrm{BaO}$ & SrO & $\mathrm{Cr}_{2} \mathrm{O}_{3}$ & $\mathrm{Cd}$ & $\mathrm{Zn}$ & $\mathrm{Pb}$ & Sb & In & $(\mathrm{OH})$ & Total & Amostra \\
\hline 0 & 0,037 & 0,013 & 0,009 & 0,034 & 0,019 & 24,931 & 0 & 0,088 & 0,092 & 0 & & & & & 0 & 53,385 & 0,002 & 0 & & 74,781 & 100 & $160 \mathrm{~m}$ \\
\hline 0 & 0,009 & 0,015 & 0,052 & 0 & 0,017 & 24,142 & 0,082 & 0 & 0,062 & 0,033 & & & & & 0 & 52,554 & 0 & 0 & & 75,593 & 100,001 & $63 \mathrm{~m}$ \\
\hline 0 & 0 & 0,049 & 0,017 & 0,023 & 0,006 & 24,732 & 0,084 & 0 & 0,262 & 0,047 & & & & & 0,011 & 52,127 & 0,032 & 0 & & 74,781 & 100 & $63 \mathrm{~m}$ \\
\hline 0 & 0 & 0,124 & 0,113 & 0,033 & 0,041 & 21,992 & 0,048 & 0 & 0,09 & 0,009 & & & & & 0 & 50,6 & 0 & 0 & & 77,56 & 100,001 & $63 \mathrm{~m}$ \\
\hline 0 & 0,006 & 0,059 & 0 & 0 & 0,023 & 24,221 & 0 & 0 & 2,549 & 0,018 & & & & & 0,002 & 52,609 & 0 & 0,009 & & 73,132 & 100,003 & $63 \mathrm{~m}$ \\
\hline 0 & 0 & 0,049 & 0,047 & 0,014 & 0,032 & 24,979 & 0,036 & 0,123 & 0,117 & 0 & & & & & 0 & 51,861 & 0 & 0,01 & & 74,611 & 100,001 & $63 \mathrm{~m}$ \\
\hline 0 & 0,026 & 0 & 0,002 & 0 & 0 & 25,461 & 0,142 & 0 & 0,329 & 0,054 & & & & & 0,011 & 52,403 & 0 & 0 & & 73,987 & 100,001 & $63 \mathrm{~m}$ \\
\hline 0 & 0,033 & 0,051 & 0,01 & 0 & 0,03 & 26,122 & 0 & 0 & 0,935 & 0,066 & & & & & 0 & 51,653 & 0 & 0 & & 72,758 & 99,998 & $63 \mathrm{~m}$ \\
\hline 0 & 0 & 0,02 & 0,037 & 0 & 0,025 & 24,534 & 0 & 0 & 0,037 & 0,002 & & & & & 0 & 52,038 & 0 & 0,005 & & 75,35 & 99,999 & $63 \mathrm{~m}$ \\
\hline 0 & 0 & 0,061 & 0,042 & 0,029 & 0,045 & 24,687 & 0,048 & 0 & 0 & 0 & & & & & 0,044 & 52,076 & 0 & 0,008 & & 75,097 & \begin{tabular}{|l|}
99,999 \\
\end{tabular} & $63 \mathrm{~m}$ \\
\hline 0 & 0 & 0,028 & 0,074 & 0,043 & 0,081 & 24,894 & 0,117 & 0 & 0,115 & 0 & & & & & 0,007 & 51,651 & 0,009 & 0,003 & & 74,667 & 100,001 & $63 \mathrm{~m}$ \\
\hline 0 & 0,021 & 0,014 & 0,002 & 0 & 0,024 & 24,985 & 0 & 0,01 & 0,147 & 0 & & & & & 0,001 & 52,202 & 0,01 & 0,003 & & 74,803 & 100,001 & $63 \mathrm{~m}$ \\
\hline 0 & 0,023 & 0,006 & 0 & 0,2 & 0 & 24,767 & 0 & 0,336 & 0,002 & 0,01 & & & & & 0 & 51,38 & 0,002 & 0,004 & & 74,654 & 99,998 & $63 \mathrm{~m}$ \\
\hline 0 & 0,009 & 0,004 & 0,067 & 0,092 & 0,011 & 23,654 & 0,107 & 0 & 0,072 & 0,034 & & & & & 0 & 52,575 & 0,017 & 0 & & 75,953 & 100,001 & $63 \mathrm{~m}$ \\
\hline 2,631 & 0,058 & 0,01 & 0,819 & 0,186 & 0,916 & 24,685 & 0 & 0,11 & 0,049 & 0 & & & & & 0,02 & 51,319 & 0 & 0 & & 70,742 & 99,999 & $63 \mathrm{~m}$ \\
\hline 0 & 0,04 & 0 & 0,042 & 0,019 & 0 & 24,347 & 0,153 & 0,062 & 0,424 & 0,038 & & & & & 0,043 & 51,938 & 0 & 0,002 & & 74,874 & 99,999 & $63 \mathrm{~m}$ \\
\hline 0 & 0,001 & 0,007 & 0,003 & 0 & 0,029 & 26,491 & 0 & 0 & 0,11 & 0,042 & 0,032 & 0 & 0,072 & 0,043 & 0 & 53,041 & 0 & 0 & 0,011 & 0 & 100,695 & $172 \mathrm{~m}$ \\
\hline 0 & 0,017 & 0,01 & 0,015 & 0,035 & 0,039 & 26,576 & 0 & 0,011 & 0,045 & 0 & 0,049 & 0,153 & 0 & 0 & 0 & 52,775 & 0,324 & 0,009 & 0 & 0 & 100,524 & $172 \mathrm{~m}$ \\
\hline 0 & 0,045 & 0 & 0,001 & 0,028 & 0,002 & 26,444 & 0,04 & 0,008 & 0,058 & 0 & 0,05 & 0,056 & 0,058 & 0 & 0,015 & 53,128 & 0 & 0 & 0 & 0 & 100,906 & $172 \mathrm{~m}$ \\
\hline 0 & 0,065 & 0,004 & 0 & 0,028 & 0,01 & 26,318 & 0 & 0 & 0,14 & 0,03 & 0 & 0 & 0 & 0 & 0 & 52,799 & 0,08 & 0 & 0,007 & 0 & 100,301 & $172 \mathrm{~m}$ \\
\hline 0 & 0,044 & 0 & 0 & 0,012 & 0,019 & 26,383 & 0 & 0,036 & 0,293 & 0,031 & 0,035 & 0,056 & 0,125 & 0,034 & 0,058 & 51,118 & 0 & 0,017 & 0 & 0 & 101,242 & $172 \mathrm{~m}$ \\
\hline 0 & 0,008 & 0,055 & 0 & 0,035 & 0,023 & 26,178 & 0 & 0,038 & 0,082 & 0,034 & 0,039 & 0 & 0,076 & 0 & 0,001 & 50,925 & 0,038 & 0,007 & 0 & 0 & 100,127 & $172 \mathrm{~m}$ \\
\hline 0 & 0,01 & 0,08 & 0 & 0 & 0,031 & 26,163 & 0,098 & 0 & 0,701 & 0 & 0,101 & 0,036 & 0 & 0,043 & 0 & 50,313 & 0,05 & 0,013 & 0 & 0 & 100,359 & $172 \mathrm{~m}$ \\
\hline 0 & 0,009 & 0,021 & 0 & 0,018 & 0,033 & 26,054 & 0 & 0,061 & 0,047 & 0,011 & 0 & 0 & 0 & 0 & 0 & 51,605 & 0,043 & 0,057 & 0,002 & 0 & 100,335 & $172 \mathrm{~m}$ \\
\hline 0 & 0,001 & 0 & 0,017 & 0 & 0,018 & 26,347 & 0,07 & 0 & 0,172 & 0 & 0,029 & 0 & 0 & 0,023 & 0,013 & 51,485 & 0,145 & 0 & 0 & 0 & 101,592 & $172 \mathrm{~m}$ \\
\hline
\end{tabular}




\begin{tabular}{|c|c|c|c|c|c|c|c|c|c|c|c|c|c|c|c|c|c|c|c|c|c|c|}
\hline 0 & 0,039 & 0,028 & 0,004 & 0 & 0 & 26,619 & 0 & 0,056 & 0,005 & 0,019 & 0 & 0 & 0,025 & 0,045 & 0 & 51,461 & 0,087 & 0,019 & 0 & 0 & 100,869 & $172 \mathrm{~m}$ \\
\hline 0 & 0,059 & 0 & 0,02 & 0,008 & 0 & 26,692 & 0 & 0,014 & 0,068 & 0 & 0,057 & 0,09 & 0,123 & 0,035 & 0 & 50,547 & 0,005 & 0 & 0 & 0 & 100,966 & $172 \mathrm{~m}$ \\
\hline
\end{tabular}

Apêndice 12. Análises de química mineral (em \% de peso) de willemita da segunda fase do estágio mineralizante (estágio principal de mineralização) no Membro Pamplona Inferior.

\begin{tabular}{|c|c|c|c|c|c|c|c|c|c|c|c|c|c|c|c|c|c|c|c|c|c|c|}
\hline $\mathrm{Na}_{2} \mathrm{O}$ & $\mathrm{MgO}$ & $\mathrm{Al}_{2} \mathrm{O}_{3}$ & $\mathrm{~K}_{2} \mathrm{O}$ & $\mathrm{CaO}$ & $\mathrm{Cl}$ & $\mathrm{SiO}_{2}$ & $\mathrm{TiO}_{2}$ & $\mathrm{MnO}$ & $\mathrm{FeO}$ & $\mathrm{V}_{2} \mathrm{O}_{3}$ & $\mathrm{P}_{2} \mathrm{O}_{5}$ & $\mathrm{BaO}$ & SrO & $\mathrm{Cr}_{2} \mathrm{O}_{3}$ & Cd & $Z n$ & $\mathrm{~Pb}$ & Sb & In & $(\mathrm{OH})$ & Total & Amostra \\
\hline 0 & 0,02 & 0,022 & 0,04 & 0,049 & 0 & 25,825 & 0,146 & 0 & 0,074 & 0,036 & 0,879 & 0 & 0 & 0,011 & 0,026 & 52,269 & 0 & 0,035 & 0 & 0,158 & 99,999 & $411 \mathrm{~m}$ \\
\hline 0 & 0 & 0 & 0 & 0,05 & 0 & 25,839 & 0 & 0 & 0,023 & 0,016 & 0,477 & 0 & 0,025 & 0,058 & 0,019 & 52,729 & 0,044 & 0 & 0,009 & 0,518 & 100,001 & $411 \mathrm{~m}$ \\
\hline 0 & 0,047 & 0 & 0,019 & 0,051 & 0,021 & 25,851 & 0,075 & 0,062 & 0,096 & 0 & 0,224 & 0,064 & 0,021 & 0,022 & 0,017 & 52,085 & 0,08 & 0,044 & 0,004 & 0,886 & 99,999 & $11 \mathrm{~m}$ \\
\hline 0 & 0 & 0,012 & 0 & 0,042 & 0,006 & 26,468 & 0 & 0 & 0,079 & 0,017 & 0 & 0 & 0,114 & 0 & 0 & 51,988 & 0,021 & 0 & 0 & 1,667 & 100 & $411 \mathrm{~m}$ \\
\hline 0 & 0 & 0 & 0,011 & 0,03 & 0,014 & 26,27 & 0,097 & 0 & 0,034 & 0,017 & 0,049 & 0 & 0,097 & 0,01 & 0 & 52,501 & 0,109 & 0 & 0 & 0,668 & 100,001 & $411 \mathrm{~m}$ \\
\hline 0 & 0,019 & 0 & 0,016 & 0 & 0,008 & 27,065 & 0 & 0 & 0,09 & 0,02 & 0,043 & 0,056 & 0 & 0 & 0 & 52,125 & 0,148 & 0,045 & 0 & 0,56 & 100 & $411 \mathrm{~m}$ \\
\hline 0 & 0,016 & 0,304 & 0 & 0,054 & 0,007 & 26,585 & 0 & 0 & 0,096 & 0,023 & 0,232 & 0,037 & 0 & 0,021 & 0 & 52,035 & 0,108 & 0,026 & 0 & 1,113 & 99,999 & $411 \mathrm{~m}$ \\
\hline 0 & 0,023 & 0,212 & 0,027 & 0,075 & 0,018 & 26,319 & 0,11 & 0,045 & 0,001 & 0,018 & 0,191 & 0,032 & 0 & 0 & 0 & 51,943 & 0 & 0,026 & 0 & 2,42 & 100,003 & 411 m \\
\hline 0 & 0,038 & 0 & 0 & 0,216 & 0,035 & 26,83 & 0,005 & 0,013 & 0,147 & 0,004 & 0,363 & 0,08 & 0 & 0 & 0,008 & 52,036 & 0 & 0,047 & 0,003 & 0,508 & 100 & $411 \mathrm{~m}$ \\
\hline
\end{tabular}


Apêndice 13. Análises de química mineral (em \% de peso) de esfalerita da alteração de pirita no Membro Morro do Pinheiro Superior.

\begin{tabular}{|c|c|c|c|c|c|c|c|c|c|c|c|c|c|c|}
\hline As & Ge & $\mathrm{Ga}$ & $\mathrm{Fe}$ & $\mathrm{Cu}$ & $\mathrm{Zn}$ & Cd & $\mathbf{B i}$ & $\mathrm{Au}$ & $S$ & $\mathrm{~Pb}$ & $\mathrm{Ag}$ & Sb & Total & Amostra \\
\hline 0 & 0 & 0 & 0,991 & 0,126 & 66,199 & 0,126 & 0 & 0 & 32,609 & 0,074 & 0,004 & 0 & 100,129 & $615 \mathrm{~m}$ \\
\hline 0 & 0 & 0 & 0,882 & 0,051 & 65,728 & 0,127 & 0 & 0 & 32,653 & 0,07 & 0,007 & 0 & 99,518 & 615 m \\
\hline 0 & 0 & 0 & 0,881 & 0,062 & 66,35 & 0,178 & 0 & 0 & 32,799 & 0,042 & 0 & 0,015 & 100,327 & $615 \mathrm{~m}$ \\
\hline 0 & 0 & 0 & 0,94 & 0,069 & 65,215 & 0,163 & 0 & 0 & 32,839 & 0,188 & 0 & 0 & 99,414 & $615 \mathrm{~m}$ \\
\hline 0 & 0 & 0 & 1,637 & 0,84 & 64,247 & 0,186 & 0 & 0 & 32,821 & 0,058 & 0 & 0 & 99,789 & $615 \mathrm{~m}$ \\
\hline 0 & 0 & 0 & 0,907 & 0,005 & 65,37 & 0,115 & 0 & 0 & 32,95 & 0,044 & 0 & 0 & 99,391 & $615 \mathrm{~m}$ \\
\hline 0 & 0 & 0 & 0,926 & 0,058 & 65,063 & 0,089 & 0 & 0 & 32,632 & 0,034 & 0 & 0 & 98,802 & $615 \mathrm{~m}$ \\
\hline 0 & 0 & 0 & 0,887 & 0,108 & 64,609 & 0,141 & 0 & 0 & 32,567 & 0,066 & 0 & 0 & 98,378 & $615 \mathrm{~m}$ \\
\hline 0 & 0 & 0 & 0,837 & 0,046 & 64,878 & 0,104 & 0 & 0 & 32,921 & 0,085 & 0 & 0 & 98,871 & $615 \mathrm{~m}$ \\
\hline 0 & 0 & 0 & 0,935 & 0,072 & 64,788 & 0,116 & 0 & 0 & 32,491 & 0,056 & 0 & 0 & 98,458 & $615 \mathrm{~m}$ \\
\hline 0 & 0 & 0 & 0,884 & 0 & 65,645 & 0,177 & 0 & 0 & 32,947 & 0 & 0 & 0 & 99,653 & $615 \mathrm{~m}$ \\
\hline 0 & 0 & 0 & 1,162 & 0,364 & 65,298 & 0,137 & 0 & 0 & 33,156 & 0,082 & 0 & 0 & 100,199 & $615 \mathrm{~m}$ \\
\hline 0 & 0 & 0 & 0,94 & 0,026 & 65,303 & 0,109 & 0 & 0 & 32,885 & 0,069 & 0,018 & 0 & 99,35 & $615 \mathrm{~m}$ \\
\hline 0 & 0 & 0 & 0,922 & 0,111 & 65,669 & 0,093 & 0 & 0 & 32,761 & 0,127 & 0 & 0 & 99,683 & $615 \mathrm{~m}$ \\
\hline 0 & 0 & 0 & 0,876 & 0 & 65,25 & 0,125 & 0 & 0 & 32,83 & 0,052 & 0 & 0 & 99,133 & $615 \mathrm{~m}$ \\
\hline 0 & 0 & 0 & 1,183 & 0,405 & 64,258 & 0,188 & 0 & 0 & 32,893 & 0,074 & 0,015 & 0 & 99,016 & $615 \mathrm{~m}$ \\
\hline 0 & 0 & 0 & 0,814 & 0,041 & 64,577 & 0,147 & 0 & 0 & 32,912 & 0,1 & 0 & 0 & 98,591 & $615 \mathrm{~m}$ \\
\hline 0 & 0 & 0 & 0,803 & 0,006 & 64,301 & 0,176 & 0 & 0 & 32,713 & 0,009 & 0 & 0 & 98,008 & $615 \mathrm{~m}$ \\
\hline 0 & 0 & 0 & 1,106 & 0,361 & 64,61 & 0,113 & 0 & 0 & 32,548 & 0,141 & 0 & 0 & 98,879 & $615 \mathrm{~m}$ \\
\hline 0 & 0 & 0 & 0,929 & 0,057 & 65,011 & 0,158 & 0 & 0 & 32,53 & 0,031 & 0,003 & 0 & 98,719 & $615 \mathrm{~m}$ \\
\hline 0 & 0 & 0 & 0,835 & 0,038 & 64,97 & 0,148 & 0 & 0 & 32,466 & 0,087 & 0 & 0 & 98,544 & $615 \mathrm{~m}$ \\
\hline 0 & 0 & 0 & 0,877 & 0,01 & 65,154 & 0,175 & 0 & 0 & 32,94 & 0,048 & 0 & 0 & 99,204 & $615 \mathrm{~m}$ \\
\hline 0 & 0 & 0 & 0,816 & 0,057 & 65,143 & 0,162 & 0 & 0 & 32,872 & 0,06 & 0 & 0 & 99,11 & $615 \mathrm{~m}$ \\
\hline 0 & 0 & 0 & 0,888 & 0,011 & 63 & 0,07 & 0 & 0 & 31,755 & 0,102 & 0,003 & 0 & 95,829 & $615 \mathrm{~m}$ \\
\hline 0 & 0 & 0 & 0,854 & 0 & 65,016 & 0,19 & 0 & 0 & 32,632 & 0,069 & 0 & 0 & 98,761 & $615 \mathrm{~m}$ \\
\hline
\end{tabular}




\begin{tabular}{|c|c|c|c|c|c|c|c|c|c|c|c|c|c|c|}
\hline 0 & 0 & 0 & 0,869 & 0,011 & 64,928 & 0,139 & 0 & 0 & 32,741 & 0,061 & 0 & 0 & 98,749 & $615 \mathrm{~m}$ \\
\hline 0 & 0 & 0 & 0,939 & 0,017 & 65,21 & 0,147 & 0 & 0 & 32,501 & 0,062 & 0 & 0 & 98,876 & $615 \mathrm{~m}$ \\
\hline 0 & 0 & 0 & 1,07 & 0,243 & 64,455 & 0,086 & 0 & 0 & 33,002 & 0,053 & 0 & 0 & 98,909 & $615 \mathrm{~m}$ \\
\hline 0 & 0 & 0 & 0,884 & 0,031 & 64,853 & 0,123 & 0 & 0 & 32,886 & 0,066 & 0 & 0 & 98,843 & $615 \mathrm{~m}$ \\
\hline 0 & 0 & 0 & 1,059 & 0,209 & 64,613 & 0,117 & 0 & 0,01 & 32,527 & 0,102 & 0 & 0 & 98,637 & $615 \mathrm{~m}$ \\
\hline 0 & 0 & 0 & 0,93 & 0,083 & 64,627 & 0,138 & 0 & 0 & 32,789 & 0,129 & 0 & 0 & 98,696 & $615 \mathrm{~m}$ \\
\hline 0 & 0 & 0 & 0,919 & 0,144 & 65,118 & 0,123 & 0 & 0 & 32,86 & 0,086 & 0 & 0 & 99,25 & $615 \mathrm{~m}$ \\
\hline 0 & 0 & 0 & 0,83 & 0,014 & 63,638 & 0,14 & 0 & 0 & 32,953 & 0,094 & 0,008 & 0 & 97,677 & $615 \mathrm{~m}$ \\
\hline 0 & 0 & 0 & 0,956 & 0 & 65,416 & 0,109 & 0 & 0 & 32,582 & 0,071 & 0 & 0 & 99,134 & $615 \mathrm{~m}$ \\
\hline 0 & 0 & 0 & 1,422 & 0,84 & 63,999 & 0,111 & 0 & 0 & 32,824 & 0,086 & 0 & 0 & 99,282 & $615 \mathrm{~m}$ \\
\hline 0 & 0 & 0 & 0,529 & 0,092 & 65,413 & 0,165 & 0 & 0 & 32,786 & 0,142 & 0 & 0 & 99,127 & $615 \mathrm{~m}$ \\
\hline 0 & 0 & 0 & 0,61 & 0,145 & 65,06 & 0,145 & 0 & 0 & 32,845 & 0,059 & 0 & 0 & 98,864 & $615 \mathrm{~m}$ \\
\hline 0 & 0 & 0 & 0,647 & 0,228 & 64,636 & 0,144 & 0 & 0 & 32,756 & 0,076 & 0 & 0 & 98,487 & $615 \mathrm{~m}$ \\
\hline 0 & 0 & 0 & 0,81 & 0,455 & 63,463 & 0,213 & 0 & 0 & 32,613 & 0,109 & 0 & 0 & 97,663 & $615 \mathrm{~m}$ \\
\hline 0 & 0 & 0 & 1,138 & 0,332 & 64,104 & 0,162 & 0 & 0 & 32,521 & 0,111 & 0 & 0 & 98,368 & $615 \mathrm{~m}$ \\
\hline 0 & 0 & 0 & 1,164 & 0,549 & 63,811 & 0,218 & 0 & 0 & 32,686 & 0,165 & 0 & 0 & 98,593 & $615 \mathrm{~m}$ \\
\hline 0 & 0 & 0 & 1,043 & 0,293 & 63,964 & 0,203 & 0 & 0 & 32,766 & 0,122 & 0 & 0 & 98,391 & $615 \mathrm{~m}$ \\
\hline 0 & 0 & 0 & 1,113 & 0,387 & 64,785 & 0,197 & 0 & 0 & 32,483 & 0,026 & 0 & 0 & 98,991 & $615 \mathrm{~m}$ \\
\hline 0 & 0 & 0 & 1,006 & 0,185 & 65,137 & 0,176 & 0 & 0 & 32,773 & 0,078 & 0,02 & 0 & 99,375 & $615 \mathrm{~m}$ \\
\hline 0 & 0 & 0 & 0,991 & 0,176 & 65,233 & 0,188 & 0 & 0 & 32,559 & 0,15 & 0 & 0 & 99,297 & $615 \mathrm{~m}$ \\
\hline 0 & 0 & 0 & 1,06 & 0,13 & 65,566 & 0,141 & 0 & 0 & 32,926 & 0,039 & 0 & 0 & 99,862 & $615 \mathrm{~m}$ \\
\hline 0 & 0 & 0 & 1,171 & 0,457 & 64,204 & 0,174 & 0 & 0 & 32,77 & 0,117 & 0 & 0 & 98,893 & $615 \mathrm{~m}$ \\
\hline 0 & 0 & 0 & 1,142 & 0,348 & 64,82 & 0,139 & 0 & 0 & 32,72 & 0,084 & 0,003 & 0 & 99,256 & $615 \mathrm{~m}$ \\
\hline 0 & 0 & 0 & 1,424 & 0 & 63,121 & 0,384 & 0 & 0 & 32,118 & 0,049 & 0 & 0 & 97,096 & $603 \mathrm{~m}$ \\
\hline
\end{tabular}


Apêndice 14. Análises de química mineral (em \% de peso) de esfalerita da alteração de galena no Membro Pamplona Inferior.

\begin{tabular}{|c|c|c|c|c|c|c|c|c|c|c|c|c|c|c|}
\hline As & $\mathrm{Ge}$ & Ga & $\mathrm{Fe}$ & $\mathrm{Cu}$ & $\mathrm{Zn}$ & $\mathrm{Cd}$ & $\mathrm{Bi}$ & $\mathrm{Au}$ & $S$ & $\mathrm{~Pb}$ & $\mathrm{Ag}$ & Sb & Total & Amostra \\
\hline 0 & 0 & 0 & 0,13 & 0,082 & 64,269 & 0,462 & 0 & 0 & 32,013 & 0,102 & 0 & 0 & 97,058 & $411 \mathrm{~m}$ \\
\hline 0 & 0 & 0 & 0,284 & 0,186 & 64,735 & 0,436 & 0 & 0 & 32,755 & 0,063 & 0 & 0 & 98,459 & $411 \mathrm{~m}$ \\
\hline 0 & 0 & 0 & 0,176 & 0,065 & 64,074 & 0,452 & 0 & 0 & 31,997 & 0,221 & 0 & 0 & 96,985 & $411 \mathrm{~m}$ \\
\hline 0 & 0 & 0 & 0,152 & 0,004 & 65,89 & 0,484 & 0 & 0 & 32,654 & 0,045 & 0 & 0 & 99,229 & $411 \mathrm{~m}$ \\
\hline 0 & 0 & 0 & 0,183 & 0,118 & 65,613 & 0,497 & 0 & 0 & 32,728 & 0,053 & 0 & 0 & 99,192 & $411 \mathrm{~m}$ \\
\hline
\end{tabular}

Apêndice 15. Análises de química mineral (em \% de peso) de pirita da alteração de pirita no Membro Morro do Pinheiro Superior.

\begin{tabular}{|c|c|c|c|c|c|c|c|c|c|c|c|c|c|c|}
\hline As & Ge & Ga & $\mathrm{Fe}$ & $\mathrm{Cu}$ & $\mathrm{Zn}$ & Cd & $\mathbf{B i}$ & $\mathrm{Au}$ & $S$ & $\mathrm{~Pb}$ & $\mathrm{Ag}$ & $\mathrm{Sb}$ & Total & Amostra \\
\hline 0 & 0 & 0 & 46,633 & 0,062 & 0 & 0,009 & 0 & 0 & 53,749 & 0,12 & 0,008 & 0 & 100,581 & $615 \mathrm{~m}$ \\
\hline 0 & 0 & 0 & 46,257 & 0,086 & 0,034 & 0,011 & 0 & 0 & 53,557 & 0,111 & 0 & 0 & 100,056 & $615 \mathrm{~m}$ \\
\hline 0 & 0 & 0 & 46,493 & 0 & 0 & 0,003 & 0 & 0 & 53,605 & 0,103 & 0,001 & 0 & 100,205 & $615 \mathrm{~m}$ \\
\hline 0 & 0 & 0 & 46,814 & 0,053 & 0 & 0 & 0 & 0 & 53,656 & 0,092 & 0,007 & 0 & 100,622 & $615 \mathrm{~m}$ \\
\hline 0 & 0 & 0 & 46,603 & 0,079 & 0,011 & 0,008 & 0 & 0 & 53,369 & 0,094 & 0 & 0 & 100,164 & $615 \mathrm{~m}$ \\
\hline 0 & 0 & 0 & 46,573 & 0,008 & 0 & 0,04 & 0 & 0 & 53,483 & 0,038 & 0,004 & 0,012 & 100,158 & $615 \mathrm{~m}$ \\
\hline 0 & 0 & 0 & 46,395 & 0,035 & 0,02 & 0 & 0 & 0 & 53,605 & 0,105 & 0,006 & 0,001 & 100,167 & $615 \mathrm{~m}$ \\
\hline 0 & 0 & 0 & 46,547 & 0,011 & 0,044 & 0,025 & 0 & 0 & 53,563 & 0,09 & 0,001 & 0 & 100,281 & $615 \mathrm{~m}$ \\
\hline 0 & 0 & 0 & 46,032 & 0,055 & 0,003 & 0 & 0 & 0 & 53,666 & 0,124 & 0 & 0,006 & 99,886 & $615 \mathrm{~m}$ \\
\hline 0,72 & 0 & 0 & 46,272 & 0,06 & 0 & 0 & 0 & 0 & 53,188 & 0,067 & 0,012 & 0 & 100,319 & $615 \mathrm{~m}$ \\
\hline 0,039 & 0 & 0 & 45,902 & 0,081 & 0,05 & 0 & 0 & 0 & 53,325 & 0,129 & 0 & 0 & 99,526 & $615 \mathrm{~m}$ \\
\hline 0 & 0 & 0 & 44,213 & 0,056 & 0 & 0 & 0 & 0 & 52,094 & 0,066 & 0,014 & 0 & 96,443 & $615 \mathrm{~m}$ \\
\hline 0 & 0 & 0 & 45,666 & 0,042 & 0,012 & 0,014 & 0 & 0 & 53,234 & 0,1 & 0 & 0 & 99,068 & $615 \mathrm{~m}$ \\
\hline 0 & 0 & 0 & 46,16 & 0,053 & 0 & 0,019 & 0 & 0 & 53,455 & 0,1 & 0,005 & 0 & 99,792 & $615 \mathrm{~m}$ \\
\hline 0,014 & 0 & 0 & 46,386 & 0,097 & 0 & 0,008 & 0 & 0 & 53,474 & 0,066 & 0 & 0 & 100,045 & $615 \mathrm{~m}$ \\
\hline 0 & 0 & 0 & 46,488 & 0,08 & 0,007 & 0 & 0 & 0 & 53,522 & 0,117 & 0,007 & 0 & 100,221 & $615 \mathrm{~m}$ \\
\hline
\end{tabular}




\begin{tabular}{|c|c|c|c|c|c|c|c|c|c|c|c|c|c|c|}
\hline 0,013 & 0 & 0 & 45,873 & 0,036 & 0 & 0,014 & 0 & 0 & 53,24 & 0,188 & 0 & 0 & 99,364 & $615 \mathrm{~m}$ \\
\hline 0 & 0 & 0 & 46,405 & 0,114 & 0 & 0,013 & 0 & 0 & 53,612 & 0,17 & 0 & 0 & 100,314 & $615 \mathrm{~m}$ \\
\hline 0,208 & 0 & 0 & 45,652 & 0,182 & 0,012 & 0 & 0 & 0 & 53,183 & 0,187 & 0 & 0 & 99,424 & $615 \mathrm{~m}$ \\
\hline 0 & 0 & 0 & 45,597 & 0,096 & 0,014 & 0,002 & 0 & 0 & 53,215 & 0,188 & 0,027 & 0 & 99,139 & $615 \mathrm{~m}$ \\
\hline 0,016 & 0 & 0 & 46,091 & 0,087 & 0,011 & 0,025 & 0 & 0 & 52,832 & 0,172 & 0 & 0 & 99,234 & $615 \mathrm{~m}$ \\
\hline 0 & 0 & 0 & 46,483 & 0,093 & 0,046 & 0,026 & 0 & 0 & 53,767 & 0,126 & 0,002 & 0 & 100,543 & $615 \mathrm{~m}$ \\
\hline 0,005 & 0 & 0 & 46,476 & 0,054 & 0,034 & 0,008 & 0 & 0 & 53,626 & 0,118 & 0 & 0 & 100,321 & $615 \mathrm{~m}$ \\
\hline 0 & 0 & 0 & 46,036 & 0,022 & 0 & 0,006 & 0 & 0 & 53,302 & 0,128 & 0,001 & 0 & 99,495 & $615 \mathrm{~m}$ \\
\hline 0 & 0 & 0 & 46,622 & 0,028 & 0,172 & 0 & 0 & 0 & 53,753 & 0,139 & 0 & 0 & 100,714 & $603 \mathrm{~m}$ \\
\hline 0 & 0 & 0 & 45,566 & 0,003 & 0 & 0 & 0 & 0 & 52,946 & 1,765 & 0,011 & 0,014 & 100,305 & $603 \mathrm{~m}$ \\
\hline 0 & 0 & 0 & 46,142 & 0,023 & 0,014 & 0,021 & 0 & 0 & 53,641 & 0,223 & 0,009 & 0 & 100,073 & $603 \mathrm{~m}$ \\
\hline 0 & 0 & 0 & 46,548 & 0 & 0 & 0 & 0 & 0 & 53,593 & 0,231 & 0,001 & 0,002 & 100,375 & $603 \mathrm{~m}$ \\
\hline 0 & 0 & 0 & 46,436 & 0,025 & 0,02 & 0 & 0 & 0 & 53,637 & 0,116 & 0 & 0,011 & 100,245 & $603 \mathrm{~m}$ \\
\hline 0,018 & 0 & 0 & 46,92 & 0 & 0,006 & 0 & 0 & 0 & 53,866 & 0,113 & 0,002 & 0 & 100,925 & $603 \mathrm{~m}$ \\
\hline 0 & 0 & 0 & 46,278 & 0,011 & 0,024 & 0 & 0 & 0 & 53,715 & 0,222 & 0,021 & 0 & 100,271 & $603 \mathrm{~m}$ \\
\hline 0,003 & 0 & 0 & 46,441 & 0 & 0,026 & 0,033 & 0 & 0 & 53,693 & 0,17 & 0 & 0,018 & 100,384 & $603 \mathrm{~m}$ \\
\hline 0 & 0 & 0 & 46,256 & 0,009 & 0 & 0,021 & 0 & 0 & 53,793 & 0,159 & 0,001 & 0 & 100,239 & $603 \mathrm{~m}$ \\
\hline 0 & 0 & 0 & 46,308 & 0,01 & 0 & 0 & 0 & 0 & 53,815 & 0,08 & 0 & 0,016 & 100,229 & $603 \mathrm{~m}$ \\
\hline 0 & 0 & 0 & 46,562 & 0,023 & 0 & 0,003 & 0 & 0 & 54,302 & 0,109 & 0,013 & 0 & 101,012 & $603 \mathrm{~m}$ \\
\hline 0,001 & 0 & 0,009 & 46,484 & 0,004 & 0 & 0,034 & 0 & 0 & 53,752 & 0,045 & 0 & 0,043 & 100,372 & $603 \mathrm{~m}$ \\
\hline 0 & 0 & 0 & 46,332 & 0,009 & 0,015 & 0,019 & 0 & 0 & 53,711 & 0,178 & 0 & 0 & 100,264 & $603 \mathrm{~m}$ \\
\hline 0 & 0 & 0 & 46,499 & 0 & 0,011 & 0 & 0 & 0 & 53,699 & 0,13 & 0 & 0 & 100,339 & $603 \mathrm{~m}$ \\
\hline
\end{tabular}




\begin{tabular}{|c|c|c|c|c|c|c|c|c|c|c|c|c|c|c|}
\hline 0 & 0 & 0 & 46,039 & 0,024 & 0 & 0 & 0 & 0 & 53,653 & 0,196 & 0 & 0 & 99,912 & $603 \mathrm{~m}$ \\
\hline 0 & 0 & 0 & 46,488 & 0,011 & 0 & 0,028 & 0 & 0 & 53,63 & 0,158 & 0 & 0 & 100,315 & $603 \mathrm{~m}$ \\
\hline 0 & 0 & 0 & 45,858 & 0,023 & 0,035 & 0 & 0 & 0 & 53,798 & 0,137 & 0 & 0 & 99,851 & $603 \mathrm{~m}$ \\
\hline 0 & 0 & 0 & 46,542 & 0,004 & 0 & 0 & 0 & 0 & 53,623 & 0,13 & 0 & 0 & 100,299 & $603 \mathrm{~m}$ \\
\hline
\end{tabular}

Apêndice 16. Análises de química mineral (em \% de peso) de pirita da alteração de pirita no Membro Pamplona Inferior.

\begin{tabular}{|c|c|c|c|c|c|c|c|c|c|c|c|c|c|c|}
\hline As & $\mathrm{Ge}$ & $\mathbf{G a}$ & $\mathrm{Fe}$ & $\mathrm{Cu}$ & $\mathrm{Zn}$ & Cd & $\mathbf{B i}$ & $\mathrm{Au}$ & $S$ & $\mathrm{~Pb}$ & $\mathrm{Ag}$ & $\mathrm{Sb}$ & Total & Amostra \\
\hline 0 & 0 & 0 & 46,562 & 0 & 0,006 & 0,035 & 0 & 0 & 53,696 & 0,13 & 0,011 & 0 & 100,44 & $273 \mathrm{~m}$ \\
\hline 0 & 0 & 0 & 46,031 & 0 & 0 & 0 & 0 & 0 & 53,538 & 0,085 & 0,003 & 0 & 99,657 & $273 \mathrm{~m}$ \\
\hline 0 & 0 & 0 & 46,503 & 0,034 & 0,032 & 0 & 0 & 0 & 53,534 & 0,077 & 0,016 & 0,016 & 100,212 & $273 \mathrm{~m}$ \\
\hline 0 & 0 & 0 & 46,814 & 0 & 0,02 & 0,018 & 0 & 0 & 53,531 & 0,085 & 0,026 & 0 & 100,494 & $273 \mathrm{~m}$ \\
\hline 0 & 0 & 0 & 45,971 & 0 & 0 & 0,036 & 0 & 0 & 53,267 & 0,14 & 0 & 0 & 99,414 & $273 \mathrm{~m}$ \\
\hline 0 & 0 & 0 & 46,5 & 0 & 0,001 & 0,062 & 0 & 0 & 53,62 & 0,072 & 0 & 0 & 100,255 & $273 \mathrm{~m}$ \\
\hline 0 & 0,003 & 0 & 46,04 & 0 & 0 & 0 & 0 & 0 & 53,522 & 0,107 & 0 & 0,011 & 99,683 & $273 \mathrm{~m}$ \\
\hline 0 & 0 & 0 & 46,278 & 0,028 & 0 & 0,015 & 0 & 0 & 53,487 & 0,1 & 0 & 0,015 & 99,923 & $273 \mathrm{~m}$ \\
\hline 0 & 0 & 0 & 46,509 & 0 & 0 & 0 & 0 & 0 & 53,685 & 0,077 & 0 & 0,014 & 100,285 & $273 \mathrm{~m}$ \\
\hline 0 & 0 & 0 & 46,473 & 0,025 & 0 & 0,048 & 0 & 0 & 53,813 & 0,124 & 0 & 0 & 100,483 & $273 \mathrm{~m}$ \\
\hline 0,007 & 0 & 0 & 46,054 & 0 & 0 & 0,013 & 0 & 0 & 53,554 & 0,136 & 0 & 0 & 99,764 & $273 \mathrm{~m}$ \\
\hline 0,111 & 0 & 0 & 45,582 & 0,04 & 0 & 0,035 & 0 & 0 & 53,296 & 0,174 & 0 & 0 & 99,238 & $273 \mathrm{~m}$ \\
\hline 0 & 0 & 0 & 46,379 & 0,021 & 0 & 0,006 & 0 & 0 & 53,496 & 0,083 & 0,001 & 0 & 99,986 & $273 \mathrm{~m}$ \\
\hline 0 & 0,002 & 0 & 46,272 & 0 & 0,021 & 0 & 0 & 0 & 53,211 & 0,093 & 0,015 & 0 & 99,614 & $273 \mathrm{~m}$ \\
\hline 0 & 0 & 0 & 45,999 & 0,02 & 0 & 0,001 & 0 & 0 & 53,488 & 0,133 & 0,024 & 0,007 & 99,672 & $273 \mathrm{~m}$ \\
\hline 0,014 & 0 & 0 & 46,053 & 0,4 & 0,014 & 0,011 & 0 & 0 & 53,201 & 0,161 & 0 & 0 & 99,854 & $273 \mathrm{~m}$ \\
\hline
\end{tabular}


Apêndice 17. Características petrográficas e microtermométricas das inclusões fluidas estudadas, Class, = classificação; Dol = dolomita; equiv, = equivalente; esc, = escura; I,F, = inclusão fluida; inc, = incolor; lev, e, = levemente escurecida; $\mathbf{P}=$ primária; $Q t z=$ quartz; Te = temperatura eutética; $\mathbf{T f}($ gelo $)=$ temperatura final de fusão de gelo; Th = temperatura de homogeneização.

\begin{tabular}{|c|c|c|c|c|c|c|c|c|c|c|c|c|c|}
\hline $\begin{array}{c}\text { Tipo de } \\
\text { Inclusão }\end{array}$ & Amostra & Campo & $\mathbf{I}, \mathbf{F}$, & Tamanho & Cor & $\begin{array}{c}\mathbf{N}^{\circ} \\
\text { fases }\end{array}$ & $\begin{array}{c}\text { Vg } \\
(\%)\end{array}$ & $\begin{array}{c}\text { Vs } \\
(\%)\end{array}$ & Class, & $\begin{array}{c}\mathbf{T e} \\
\left({ }^{\circ} \mathbf{C}\right)\end{array}$ & $\begin{array}{c}\text { Tf(gelo) } \\
\left({ }^{\circ} \mathrm{C}\right)\end{array}$ & $\begin{array}{c}\text { \% peso } \\
\mathrm{NaCl} \\
\text { equiv, }\end{array}$ & $\begin{array}{c}\text { Th } \\
\left({ }^{\circ} \mathbf{C}\right)\end{array}$ \\
\hline \multirow[t]{5}{*}{ Tipo 1} & \multirow[t]{5}{*}{$\begin{array}{c}305,40 \\
\mathrm{~m}\end{array}$} & \multirow{5}{*}{$\begin{array}{c}\text { Dol } 1 \\
\text { Campo } \\
2\end{array}$} & $\mathrm{I}, 1$ & $4 \mu \mathrm{m}$ & inc, & Bifas, & 5 & 0 & $\mathrm{P}$ & $-53,1$ & $-11,9$ & 15,86 & 81 \\
\hline & & & II, 2 & $5 \mu \mathrm{m}$ & inc, & Bifas, & 5 & 0 & $\mathrm{P}$ & & $-12,3$ & 16,24 & \\
\hline & & & II,4 & $5 \mu \mathrm{m}$ & inc, & Bifas, & 5 & 0 & $\mathrm{P}$ & $-53,1$ & & & \\
\hline & & & III, 2 & $4 \mu \mathrm{m}$ & inc, & Bifas, & 5 & 0 & $\mathrm{P}$ & $-53,1$ & & & 97 \\
\hline & & & II, 1 & $4 \mu \mathrm{m}$ & inc, & Bifas, & 5 & 0 & $\mathrm{P}$ & $-54,9$ & $-9,9$ & 13,83 & 75 \\
\hline \multirow[t]{27}{*}{ Tipo 2} & \multirow[t]{10}{*}{$\begin{array}{c}363,73 \\
\mathrm{~m}\end{array}$} & \multirow{5}{*}{$\begin{array}{c}\text { Qtz 1 } \\
\text { Campo } \\
4\end{array}$} & $\mathrm{~V}, 11$ & $3 \mu \mathrm{m}$ & $\begin{array}{c}\text { lev, } \\
\mathrm{e},\end{array}$ & Bifas, & 5 & 0 & $\mathrm{P}$ & $-53,7$ & $-16,9$ & 20,15 & 119 \\
\hline & & & $\mathrm{V}, 12$ & $3 \mu \mathrm{m}$ & inc, & Bifas, & 5 & 0 & $\mathrm{P}$ & $-53,7$ & $-16,5$ & 19,84 & \\
\hline & & & $\mathrm{V}, 13$ & $2 \mu \mathrm{m}$ & $\begin{array}{c}\text { lev, } \\
\mathrm{e},\end{array}$ & Bifas, & 5 & 0 & $\mathrm{P}$ & $-53,7$ & $-16,7$ & 19,99 & \\
\hline & & & $\mathrm{X}, 1$ & $2 \mu \mathrm{m}$ & inc, & Bifas, & 5 & 0 & $\mathrm{P}$ & $-53,9$ & $-16,3$ & 19,68 & \\
\hline & & & $X, 4$ & $4 \mu \mathrm{m}$ & inc, & Bifas, & 5 & 0 & $\mathrm{P}$ & $-52,2$ & $-16,9$ & 20,15 & \\
\hline & & \multirow{5}{*}{$\begin{array}{c}\text { Qtz 1 } \\
\text { Campo } \\
6\end{array}$} & $\mathrm{I}, 1$ & $2 \mu \mathrm{m}$ & inc, & Bifas, & 5 & 0 & $\mathrm{P}$ & $-52,1$ & $-14,4$ & 18,13 & 117 \\
\hline & & & $\mathrm{I}, 2$ & $3 \mu \mathrm{m}$ & inc, & Bifas, & 5 & 0 & $\mathrm{P}$ & $-54,4$ & $-13,4$ & 17,26 & \\
\hline & & & $\mathrm{I}, 3$ & $2 \mu \mathrm{m}$ & inc, & Bifas, & 5 & 0 & $\mathrm{P}$ & $-53,4$ & $-17,1$ & 20,30 & \\
\hline & & & II, 2 & $2 \mu \mathrm{m}$ & inc, & Bifas, & 5 & 0 & $\mathrm{P}$ & -54 & $-14,3$ & 18,04 & \\
\hline & & & II,3 & $3 \mu \mathrm{m}$ & inc, & Bifas, & 5 & 0 & $\mathrm{P}$ & $-53,4$ & $-16,2$ & 19,60 & 109 \\
\hline & \multirow[t]{8}{*}{$\begin{array}{c}363,73 \\
\mathrm{~m}\end{array}$} & \multirow{7}{*}{$\begin{array}{c}\text { Qtz 1 } \\
\text { Campo } \\
6 \\
\text { Qtz 1 } \\
\text { Campo } \\
6\end{array}$} & $\mathrm{~V}, 14$ & $1,5 \mu \mathrm{m}$ & inc, & Bifas, & 5 & 0 & $\mathrm{P}$ & & $-16,1$ & 19,53 & \\
\hline & & & $\mathrm{V}, 15$ & $2 \mu \mathrm{m}$ & inc, & Bifas, & 5 & 0 & $\mathrm{P}$ & & $-16,1$ & 19,53 & \\
\hline & & & VI,5 & $2 \mu \mathrm{m}$ & $\begin{array}{c}\text { lev, } \\
\mathrm{e},\end{array}$ & Bifas, & 5 & 0 & $\mathrm{P}$ & & $-15,8$ & 19,29 & \\
\hline & & & $\mathrm{X}, 2$ & $3 \mu \mathrm{m}$ & inc, & Bifas, & 5 & 0 & $\mathrm{P}$ & & $-16,3$ & 19,68 & \\
\hline & & & $X, 3$ & $3 \mu \mathrm{m}$ & inc, & Bifas, & 5 & 0 & $\mathrm{P}$ & & $-15,5$ & 19,05 & \\
\hline & & & $\mathrm{I}, 4$ & $4 \mu \mathrm{m}$ & inc, & Bifas, & 5 & 0 & $\mathrm{P}$ & & $-16,9$ & 20,15 & \\
\hline & & & II, 1 & $2 \mu \mathrm{m}$ & inc, & Bifas, & 5 & 0 & $\mathrm{P}$ & & $-15,5$ & 19,05 & \\
\hline & & \multirow{10}{*}{$\begin{array}{c}\text { Dol 1 } \\
\text { Campo } \\
2 \\
\\
\text { Qtz 1 } \\
\text { Campo } \\
4\end{array}$} & II;4 & $2 \mu \mathrm{m}$ & inc, & Bifas, & 5 & 0 & $\mathrm{P}$ & & $-16,9$ & 20,15 & \\
\hline & \multirow[t]{9}{*}{$\begin{array}{c}363,73 \\
\mathrm{~m}\end{array}$} & & XI,5 & $2 \mu \mathrm{m}$ & inc, & Bifas, & 5 & 0 & $\mathrm{P}$ & & & & 109 \\
\hline & & & $\mathrm{XI}, 7$ & $3 \mu \mathrm{m}$ & inc, & Bifas, & 5 & 0 & $\mathrm{P}$ & & & & 115 \\
\hline & & & $\mathrm{XI}, 8$ & $4 \mu \mathrm{m}$ & inc, & Bifas, & 5 & 0 & $\mathrm{P}$ & & & & 115 \\
\hline & & & XII,1 & $2 \mu \mathrm{m}$ & inc, & Bifas, & 5 & 0 & $\mathrm{P}$ & & & & 120 \\
\hline & & & $\mathrm{XII}, 2$ & $2 \mu \mathrm{m}$ & $\begin{array}{c}\text { lev, } \\
\mathrm{e},\end{array}$ & Bifas, & 5 & 0 & $\mathrm{P}$ & & & & 115 \\
\hline & & & XII,3 & $2 \mu \mathrm{m}$ & inc, & Bifas, & 5 & 0 & $\mathrm{P}$ & & & & 115 \\
\hline & & & XII,4 & $3 \mu \mathrm{m}$ & inc, & Bifas, & 5 & 0 & $\mathrm{P}$ & & & & 122 \\
\hline & & & XII,6 & $3 \mu \mathrm{m}$ & inc, & Bifas, & 10 & 0 & $\mathrm{P}$ & & & & 118 \\
\hline & & & XII,7 & $4 \mu \mathrm{m}$ & inc, & Bifas, & 5 & 0 & $\mathrm{P}$ & & & & 118 \\
\hline
\end{tabular}




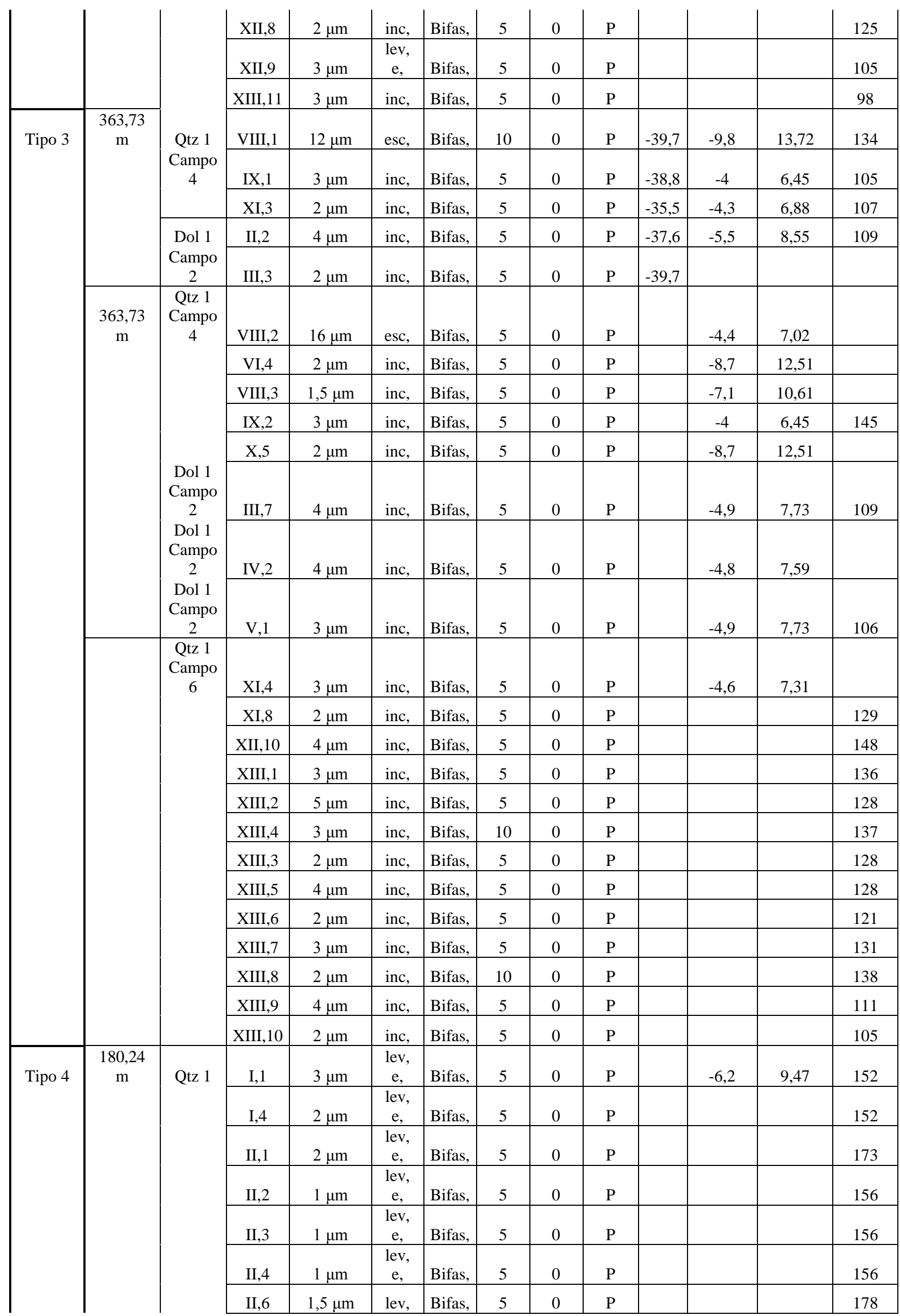




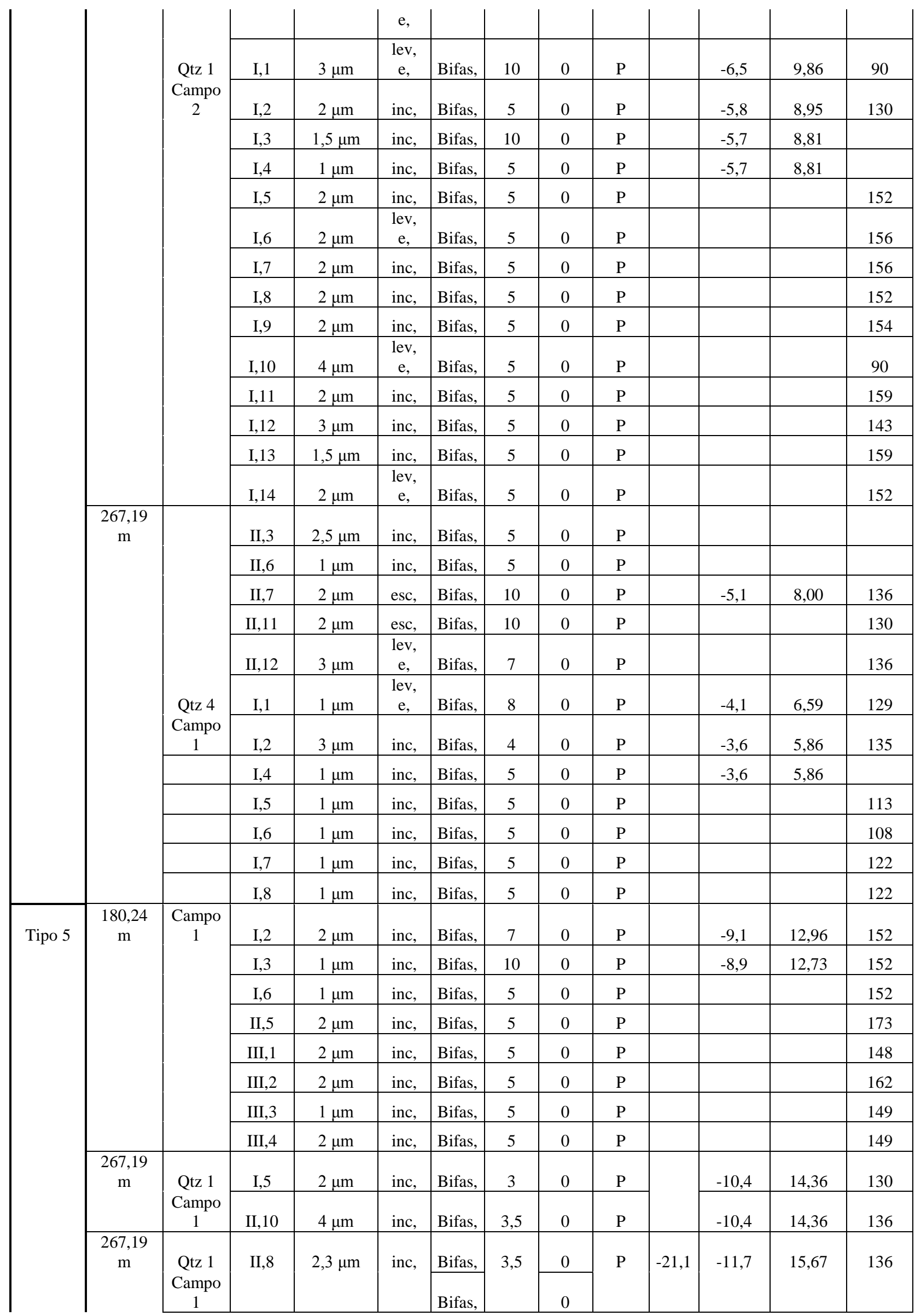




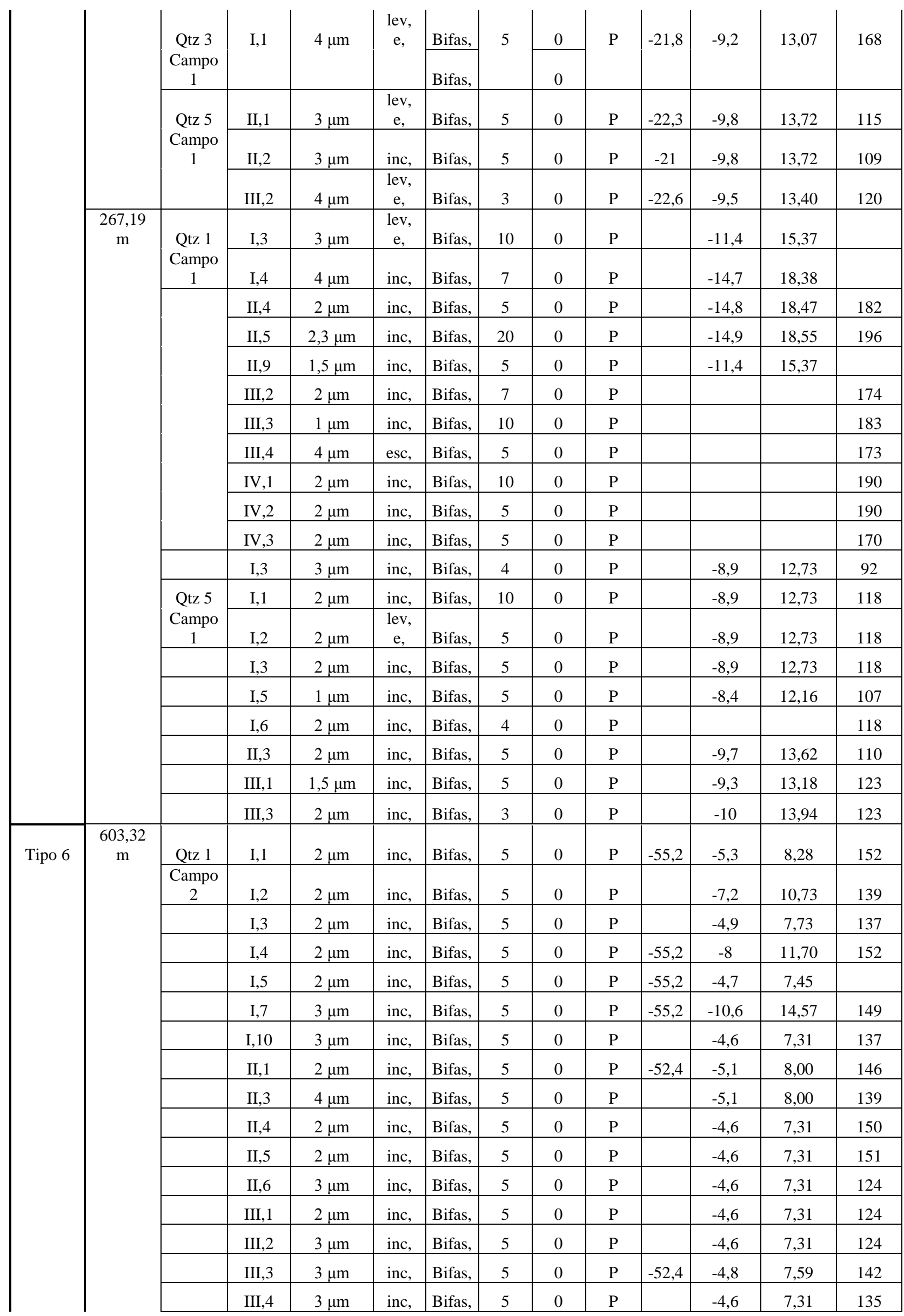




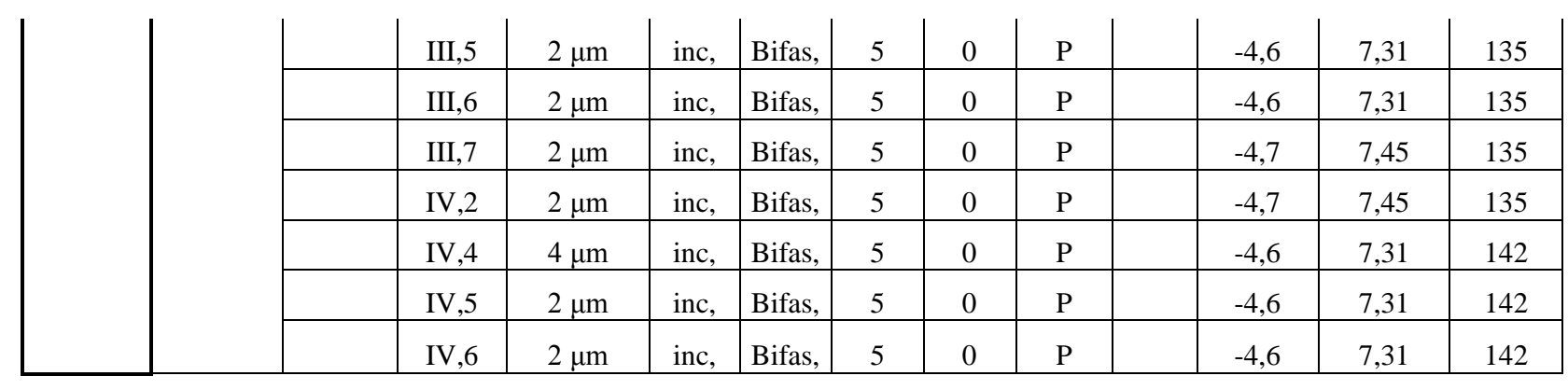


Apêndice 18. Dados de $\square^{13} \mathrm{C} \%$ e $\square^{18} \mathrm{O} \%$ para dolomita e calculados para o fluido em equilíbrio, dos vários estilos de alteração estudados.

\begin{tabular}{|c|c|c|c|c|c|c|}
\hline Estilo de Alteração & Amostra & $\begin{array}{c}\delta^{13} \mathrm{C} \% \\
\mathrm{PDB}\end{array}$ & $\begin{array}{l}\delta^{18} \mathrm{O} \% 0 \\
\mathrm{PDB}\end{array}$ & $\begin{array}{l}\delta^{18} \mathrm{O} \% \\
\text { SMOW }\end{array}$ & $\begin{array}{c}\delta^{13} \mathrm{C} \% \\
\text { PDB } \\
\text { Fluido }\end{array}$ & $\begin{array}{l}\delta^{18} \mathrm{O} \% \\
\text { SMOW } \\
\text { Fluido } \\
\end{array}$ \\
\hline \multirow[t]{19}{*}{ Dolomito Inalterado } & 127,67 hospedeira & 2,88 & $-5,64$ & 25,04 & $*$ & $*$ \\
\hline & 141,42 hospedeira & 2,16 & $-2,61$ & 28,16 & $*$ & * \\
\hline & 163,97 & 1,04 & $-6,15$ & 24,52 & $*$ & * \\
\hline & $\begin{array}{c}172,04 \text { frag brecha } r \\
\text { hospedeira }\end{array}$ & 1,78 & $-6,86$ & 23,79 & $*$ & $*$ \\
\hline & 185,38 hospedeira & 2,36 & $-3,42$ & 27,33 & $*$ & $*$ \\
\hline & 187,01 hospedeira & 2,60 & $-4,85$ & 25,86 & $*$ & $*$ \\
\hline & 202,53 hospedeira & 2,10 & $-4,08$ & 26,65 & $*$ & $*$ \\
\hline & 204,73 hospedeira & 1,97 & $-5,57$ & 25,12 & $*$ & $*$ \\
\hline & 217,82 hospedeira & 2,56 & $-4,77$ & 25,94 & $*$ & $*$ \\
\hline & 267,19 hospedeira & 2,48 & $-3,70$ & 27,04 & $*$ & $*$ \\
\hline & 273,81 hospedeira & 2,24 & $-3,20$ & 27,56 & $*$ & $*$ \\
\hline & 275,36 hospedeira & 2,20 & $-2,56$ & 28,22 & $*$ & $*$ \\
\hline & 295,54 hospedeira & 2,14 & $-2,82$ & 27,95 & $*$ & $*$ \\
\hline & 323,88 hospedeira & 0,79 & $-1,09$ & 29,74 & $*$ & * \\
\hline & 363,73 hospedeira & 1,87 & $-3,23$ & 27,53 & $*$ & $*$ \\
\hline & 411,13 hospedeira & 2,51 & $-3,54$ & 27,21 & $*$ & $*$ \\
\hline & 513,46 hospedeira & 1,06 & $-2,01$ & 28,79 & $*$ & $*$ \\
\hline & 615,40 hospedeira & 2,37 & $-2,93$ & 27,84 & $*$ & * \\
\hline & 620,80 hospedeira & 2,13 & $-1,98$ & 28,82 & $*$ & $*$ \\
\hline \multirow[t]{2}{*}{$\begin{array}{l}\text { Estágio Precoce } \\
\text { (Early Stage) }\end{array}$} & $\begin{array}{c}127,67 \text { dol banda de } \\
\text { substituicao }\end{array}$ & 2,50 & $-4,98$ & 25,73 & $-2,60$ & 5,03 \\
\hline & $\begin{array}{c}187,01 \text { dol banda de } \\
\text { substituicao }\end{array}$ & 2,30 & $-5,39$ & 25,30 & $-2,80$ & 4,60 \\
\hline $\begin{array}{l}\text { Temp, Fluido para } \\
\text { cálculo }=90^{\circ} \mathrm{C}\end{array}$ & $\begin{array}{l}267,19 \text { dol banda de } \\
\text { subst precoce }\end{array}$ & 1,48 & $-4,08$ & 26,65 & $-3,62$ & 5,95 \\
\hline \multirow[t]{3}{*}{$\begin{array}{l}\text { Estágio Pre- } \\
\text { Mineralização (Pre- } \\
\text { Ore Stage) }\end{array}$} & $\begin{array}{l}363,73 \text { dolomita dog } \\
\text { tooth }\end{array}$ & 1,49 & $-3,34$ & 27,41 & $-1,81$ & 10,21 \\
\hline & $\begin{array}{c}513,46 \text { dol substitui } \\
1\end{array}$ & 2,26 & $-4,11$ & 26,63 & $-1,04$ & 9,43 \\
\hline & $\begin{array}{c}513,46 \text { dol substitui } \\
2\end{array}$ & 1,89 & $-4,70$ & 26,02 & $-1,41$ & 8,82 \\
\hline $\begin{array}{l}\text { Temp, Fluido para } \\
\text { cálculo }=120^{\circ} \mathrm{C}\end{array}$ & 141,42 dol branca & 1,90 & $-3,66$ & 27,09 & $-1,41$ & 9,89 \\
\hline $\begin{array}{l}\text { Dolomita em Cela - } \\
\text { Estágio Mineralizante } \\
\text { (Ore Stage) }\end{array}$ & 267,19 dol barro & $-1,10$ & $-0,98$ & 29,85 & $-1,80$ & 17,65 \\
\hline \multirow[t]{8}{*}{$\begin{array}{l}\text { Dolomita Vermelha - } \\
\text { Estágio Mineralizante } \\
\text { (Ore Stage) }\end{array}$} & 127,67 dol vermelha & 0,26 & $-8,82$ & 21,77 & $-0,44$ & 9,57 \\
\hline & 138,60 dol vermelha & 0,36 & $-11,13$ & 19,39 & $-0,34$ & 7,19 \\
\hline & 139,32 dol vermelha & 0,95 & $-9,71$ & 20,85 & 0,25 & 8,65 \\
\hline & 155,25 dol vermelha & 0,73 & $-9,63$ & 20,93 & 0,03 & 8,73 \\
\hline & 160,42 dol vermelha & 1,19 & $-10,86$ & 19,67 & 0,49 & 7,47 \\
\hline & 180,24 dol vermelha & 1,14 & $-10,76$ & 19,77 & 0,44 & 7,57 \\
\hline & $\begin{array}{l}180,24 \times \text { dol } \\
\text { vermelha }\end{array}$ & 1,23 & $-7,80$ & 22,82 & 0,53 & 10,62 \\
\hline & 185,38 dol vermelha & 1,11 & $-9,38$ & 21,20 & 0,41 & 9,00 \\
\hline
\end{tabular}




\begin{tabular}{|c|c|c|c|c|c|c|}
\hline \multirow[b]{2}{*}{$\begin{array}{l}\text { Temp, Fluido para } \\
\text { cálculo }=180^{\circ} \mathrm{C}\end{array}$} & 187,01 dol vermelha & 0,67 & $-9,63$ & 20,93 & $-0,03$ & 8,73 \\
\hline & 217,82 dol vermelha & 1,09 & $-9,18$ & 21,40 & 0,39 & 9,20 \\
\hline \multirow{2}{*}{$\begin{array}{l}\text { Dolomita Branca - } \\
\text { Fases Iniciais do } \\
\text { Estágio Mineralizante } \\
\text { Temp, Fluido para } \\
\text { cálculo }=180^{\circ} \mathrm{C}\end{array}$} & 202,53 dol branca & 1,61 & $-3,21$ & 27,55 & 0,91 & 15,35 \\
\hline & 204,73 dol branca & 1,23 & $-4,50$ & 26,22 & 0,53 & 14,02 \\
\hline \multirow[t]{11}{*}{$\begin{array}{l}\text { Dolomita Branca - } \\
\text { Estágio Mineralizante } \\
\text { (Ore Stage) }\end{array}$} & 36,36 dol branca & 0,63 & $-12,42$ & 18,06 & $-0,07$ & 5,86 \\
\hline & 138 dol branca & 0,96 & $-11,48$ & 19,03 & 0,26 & 6,83 \\
\hline & 138,60 dol branca & 1,41 & $-9,07$ & 21,51 & 0,71 & 9,31 \\
\hline & 139,62 dol branca & 0,42 & $-10,59$ & 19,94 & $-0,28$ & 7,74 \\
\hline & 155,26 dol branca & 1,09 & $-11,52$ & 18,98 & 0,39 & 6,78 \\
\hline & 160,42 dol branca & 0,65 & $-10,53$ & 20,00 & $-0,05$ & 7,80 \\
\hline & 163,97 dol branca & 0,62 & $-9,21$ & 21,36 & $-0,08$ & 9,16 \\
\hline & 172,04 dol branca & $-1,62$ & $-13,97$ & 16,46 & $-2,32$ & 4,26 \\
\hline & 180,24 dol branca & 0,93 & $-11,60$ & 18,90 & 0,23 & 6,70 \\
\hline & 197,30 dol branca & 1,34 & $-9,72$ & 20,84 & 0,64 & 8,64 \\
\hline & 180,24 dol branca & 1,08 & $-10,38$ & 20,16 & 0,38 & 7,96 \\
\hline $\begin{array}{l}\text { Temp, Fluido para } \\
\text { cálculo }=180^{\circ} \mathrm{C}\end{array}$ & 525,41 dol branca & $-0,35$ & $-11,06$ & 19,45 & $-1,05$ & 7,25 \\
\hline \multirow[t]{5}{*}{$\begin{array}{l}\text { Dolomita Branca - } \\
\text { Fases Iniciais da } \\
\text { Alteração Sulfetada }\end{array}$} & $\begin{array}{c}411,13 \mathrm{~B} \text { dol branca } \\
\text { banda extern }\end{array}$ & 2,19 & $-3,53$ & 27,22 & 1,49 & 15,02 \\
\hline & $\begin{array}{l}603,32 \text { dol banda } \\
\text { externa laminada }\end{array}$ & 2,92 & $-0,81$ & 30,02 & 2,22 & 17,82 \\
\hline & 603,32 dol banda 2 & 2,09 & $-2,78$ & 27,99 & 1,39 & 15,79 \\
\hline & $411,13 \mathrm{~A}$ dol branca & 2,20 & $-3,02$ & 27,74 & 1,50 & 15,54 \\
\hline & 411,13 dol branca & 2,43 & $-3,18$ & 27,58 & 1,73 & 15,38 \\
\hline $\begin{array}{l}\text { Temp, Fluido para } \\
\text { cálculo }=135^{\circ} \mathrm{C}\end{array}$ & $\begin{array}{l}620,80 \text { dol branca } \\
\text { banda de } \\
\text { substituicao }\end{array}$ & 2,70 & $-2,73$ & 28,04 & 2,00 & 15,84 \\
\hline \multirow[t]{10}{*}{$\begin{array}{l}\text { Dolomita Branca - } \\
\text { Alteração Sulfetada }\end{array}$} & $\begin{array}{c}267,19 \text { dol branca } \\
\text { tardia }\end{array}$ & 2,14 & $-8,57$ & 22,02 & 1,44 & 9,82 \\
\hline & 273,81 dol branca & 1,90 & $-7,58$ & 23,05 & 1,20 & 10,85 \\
\hline & 275,36 dol branca & 1,52 & $-10,34$ & 20,20 & 0,82 & 8,00 \\
\hline & 295,54 dol branca & 1,97 & $-10,53$ & 20,01 & 1,27 & 7,81 \\
\hline & 603,32 dol banda 3 & 1,19 & $-4,97$ & 25,73 & 0,49 & 13,53 \\
\hline & 603,32 dol banda 4 & 0,52 & $-9,23$ & 21,35 & $-0,18$ & 9,15 \\
\hline & 615,40 dol branca & 1,24 & $-9,67$ & 20,90 & 0,54 & 8,70 \\
\hline & 273,81 dol branca & 2,27 & $-8,48$ & 22,12 & 1,57 & 9,92 \\
\hline & 323,88 dol branca & 0,37 & $-11,39$ & 19,12 & $-0,33$ & 6,92 \\
\hline & 615,40 dol branca & 1,62 & $-10,12$ & 20,43 & 0,92 & 8,23 \\
\hline $\begin{array}{l}\text { Temp, Fluido para } \\
\text { cálculo }=135^{\circ} \mathrm{C}\end{array}$ & $\begin{array}{c}615,40 \times \text { dol branca } \\
\text { dol branca }\end{array}$ & 1,52 & $-10,19$ & 20,35 & 0,82 & 8,15 \\
\hline
\end{tabular}


Apêndice 19. Análise de geoquímica de rocha total para amostras das várias fases de alteração hidrotermal, Elementos maiores em percentagem (de $\mathrm{Al}_{2} \mathrm{O}_{3}$ a TiO e $\mathrm{LOI}$ e elementos menores em ppm, exceto Zn, S e C, Alt, = alteração; E, Min, = estágio mineralizante; P,I, = Membro Pamplona Inferior; M,P,S, = Membro Morro do Pinheiro Superior,.

\begin{tabular}{|c|c|c|c|c|c|c|c|c|c|c|c|c|c|c|c|}
\hline Alteração & Amostra & $\mathrm{Al}_{2} \mathrm{O}_{3}$ & $\mathbf{B a O}$ & $\mathrm{CaO}$ & $\mathrm{Cr}_{2} \mathrm{O}_{3}$ & $\mathrm{Fe}_{2} \mathrm{O}_{3}$ & $\mathrm{~K}_{2} \mathrm{O}$ & MgO & $\mathrm{MnO}$ & $\mathrm{Na}_{2} \mathrm{O}$ & $\mathbf{P}_{2} \mathbf{O}_{5}$ & $\mathrm{SiO}_{2}$ & SrO & $\mathrm{TiO}_{2}$ & LOI \\
\hline Inalterada & 261,05 & 1,65 & $<0,01$ & 27,5 & $<0,01$ & 0,69 & 0,99 & 18,7 & 0,04 & $<0,01$ & 0,02 & 9,36 & 0,01 & 0,09 & 41,26 \\
\hline Inalterada & 338,32 & 0,43 & $<0,01$ & 31,2 & 0,03 & 0,47 & 0,32 & 21,1 & 0,05 & $<0,01$ & 0,03 & 2,18 & 0,01 & 0,01 & 45,62 \\
\hline Inalterada & 498,23 & 0,33 & $<0,01$ & 30,1 & 0,01 & 0,31 & 0,15 & 20,6 & 0,05 & $<0,01$ & 0,05 & 3,14 & 0,01 & 0,02 & 44,95 \\
\hline Inalterada & 501,52 & 1,22 & $<0,01$ & 28,6 & 0,01 & 0,79 & 0,43 & 20,2 & 0,02 & $<0,01$ & 0,01 & 4,98 & 0,01 & 0,06 & 43,68 \\
\hline Inalterada & 620,80 & 1,09 & $<0,01$ & 29,7 & $<0,01$ & 0,67 & 0,44 & 20,1 & 0,04 & $<0,01$ & 0,01 & 3,92 & 0,01 & 0,04 & 44,19 \\
\hline Pre-mineralização & 187,50 & 0,48 & $<0,01$ & 29,9 & $<0,01$ & 0,89 & 0,11 & 21 & 0,05 & $<0,01$ & 0,08 & 1,71 & 0,01 & 0,03 & 45,5 \\
\hline Pre-mineralização & 513,16 & 0,22 & $<0,01$ & 29,6 & $<0,01$ & 0,4 & 0,1 & 20,6 & 0,04 & 0,02 & 0,03 & 4,02 & 0,01 & 0,01 & 45,04 \\
\hline Pre-mineralização & 492,65 & 0,46 & $<0,01$ & 24 & 0,01 & 1,7 & 0,16 & 16,5 & 0,05 & $<0,01$ & 0,02 & 21,6 & 0,01 & 0,02 & 36,21 \\
\hline $1^{\mathrm{a}}$ fase $\mathrm{E}, \mathrm{Min}$, & 48,25 & 1,41 & $<0,01$ & 29,2 & $<0,01$ & 0,83 & 0,53 & 19,9 & 0,05 & $<0,01$ & 0,32 & 4,42 & 0,01 & 0,04 & 43,38 \\
\hline $1^{\text {a }}$ fase $\mathrm{E}, \mathrm{Min}$, & 101,00 & 1,73 & $<0,01$ & 27 & $<0,01$ & 1,24 & 0,54 & 18,4 & 0,05 & 0,01 & 0,56 & 10,3 & 0,01 & 0,06 & 40,01 \\
\hline Principal E, Min, P,I, & 172,04 & 0,26 & 0,02 & 17,6 & 0,01 & 1,36 & 0,02 & 12,1 & 0,06 & $<0,01$ & 0,04 & 12,4 & $<0,01$ & 0,02 & 27,95 \\
\hline Principal E, Min, P,I, & 181,60 & 0,08 & $<0,01$ & 29,6 & $<0,01$ & 3,23 & $<0,01$ & 20,7 & 0,03 & $<0,01$ & 0,04 & 0,19 & $<0,01$ & $<0,01$ & 45,22 \\
\hline Principal E, Min, P,I, & 183,33 & 0,17 & $<0,01$ & 19,1 & $<0,01$ & 37,52 & $<0,01$ & 13,2 & 0,06 & $<0,01$ & 0,07 & 0,47 & 0,01 & $<0,01$ & 29,11 \\
\hline Principal E, Min, P,I, & 168,88 & 0,32 & $<0,01$ & 22,5 & $<0,01$ & 24,28 & 0,02 & 15,4 & 0,1 & $<0,01$ & 0,19 & 1,2 & 0,01 & $<0,01$ & 34,27 \\
\hline Principal E, Min, P,I, & 217,24 & 0,2 & $<0,01$ & 15,8 & $<0,01$ & 32,63 & 0,01 & 10,7 & 0,07 & $<0,01$ & 0,02 & 10,9 & 0,01 & $<0,01$ & 24,35 \\
\hline Principal E, Min, P,I, & 63,36 & 0,18 & 0,02 & 11 & 0,01 & 4,92 & 0,01 & 7,33 & 0,07 & $<0,01$ & 0,05 & 17,4 & 0,01 & 0,01 & 17,23 \\
\hline Principal E, Min, P,I, & 64,24 & 0,75 & $<0,01$ & 8,44 & $<0,01$ & 66,64 & $<0,01$ & 6,08 & 0,07 & $<0,01$ & 0,14 & 1,88 & 0,01 & $<0,01$ & 13,19 \\
\hline Principal E, Min, M,P,S, & 521,41 & 0,15 & 0,03 & 6,7 & 0,01 & 12,52 & 0,01 & 4,66 & 0,04 & $<0,01$ & 0,02 & 18 & 0,01 & 0,02 & 10,26 \\
\hline Principal E, Min, M,P,S, & 531,80 & 0,15 & 0,02 & 5,63 & 0,01 & 18,53 & 0,01 & 3,81 & 0,04 & $<0,01$ & 0,19 & 17,6 & 0,01 & 0,01 & 8,37 \\
\hline Principal E, Min, M,P,S, & 568,80 & 0,25 & $<0,01$ & 10,7 & $<0,01$ & 57,72 & 0,01 & 7,28 & 0,05 & $<0,01$ & 0,02 & 2,38 & 0,01 & $<0,01$ & 16,52 \\
\hline $3^{\text {a }}$ fase E,Min, & 169,09 & 0,13 & 0,08 & 27,7 & 0,01 & 7,46 & $<0,01$ & 19 & 0,17 & $<0,01$ & 0,01 & 0,65 & 0,01 & $<0,01$ & 42,86 \\
\hline $3^{\mathrm{a}}$ fase E,Min, & 255,34 & 0,88 & $<0,01$ & 29 & $<0,01$ & 2,44 & 0,11 & 20,7 & 0,08 & $<0,01$ & 0,11 & 1,86 & 0,01 & 0,04 & 44,33 \\
\hline $3^{\text {a }}$ fase E,Min, & 562,05 & 0,26 & $<0,01$ & 29,6 & 0,02 & 0,59 & 0,05 & 20,2 & 0,14 & $<0,01$ & 0,02 & 0,54 & 0,01 & 0,01 & 45,9 \\
\hline Alt, de Pirita & 273,81 & 1,15 & $<0,01$ & 22,6 & $<0,01$ & 11,08 & 0,72 & 15,2 & 0,07 & 0,02 & 0,11 & 11,4 & 0,01 & 0,06 & 22,17 \\
\hline Alt, de Pirita & 295,48 & 0,88 & 0,38 & 27,6 & $<0,01$ & 4,28 & 0,47 & 18,9 & 0,07 & 0,01 & 0,02 & 4,23 & 0,02 & 0,04 & 37,3 \\
\hline Alt, de Pirita & 411,13 & 0,54 & $<0,01$ & 19,8 & $<0,01$ & 0,71 & 0,26 & 13,5 & 0,04 & 0,01 & 0,02 & 35,4 & 0,01 & 0,02 & 30 \\
\hline Alt, de Pirita & 603,23 & 0,29 & $<0,01$ & 26,9 & $<0,01$ & 5,43 & 0,13 & 18,1 & 0,03 & 0,01 & 0,01 & 4,24 & 0,01 & 0,01 & 35,05 \\
\hline Alt, de Pirita & 615,40 & 0,96 & $<0,01$ & 29,6 & $<0,01$ & 1,31 & 0,37 & 20,1 & 0,06 & 0,01 & 0,01 & 3,46 & 0,01 & 0,05 & 44,53 \\
\hline
\end{tabular}




\begin{tabular}{|c|c|c|c|c|c|c|c|c|c|c|c|c|c|c|c|c|c|c|c|}
\hline Alteração & Amostra & $\mathrm{Ba}$ & $\mathrm{Ce}$ & $\mathrm{Cr}$ & $\mathrm{Cs}$ & Dy & $\mathrm{Er}$ & $\mathrm{Eu}$ & $\mathrm{Ga}$ & $\mathrm{Gd}$ & $\mathrm{Ge}$ & Hf & Ho & $\mathrm{La}$ & $\mathrm{Lu}$ & $\mathrm{Nb}$ & $\mathrm{Nd}$ & $\operatorname{Pr}$ & $\mathrm{Rb}$ \\
\hline Inalterada & 261,05 & 131,5 & 8,7 & 40 & 0,46 & 0,59 & 0,37 & 0,2 & 1,9 & 0,72 & $<5$ & 1 & 0,16 & 3,9 & 0,06 & 1,8 & 3,7 & 0,99 & 16,3 \\
\hline Inalterada & 338,32 & 35 & 3,9 & 20 & 0,11 & 0,31 & 0,14 & 0,08 & 0,6 & 0,29 & $<5$ & $<0,2$ & 0,06 & 2,1 & 0,02 & 0,8 & 1,8 & 0,45 & 4,5 \\
\hline Inalterada & 498,23 & 38,6 & 2,7 & 30 & 0,19 & 0,18 & 0,15 & 0,07 & 1 & 0,21 & $<5$ & $<0,2$ & 0,05 & 1,3 & 0,03 & 0,8 & 1 & 0,3 & 5,5 \\
\hline Inalterada & 501,52 & 49,1 & 7,8 & 30 & 0,69 & 0,36 & 0,27 & 0,12 & 2,1 & 0,52 & $<5$ & $<0,2$ & 0,11 & 4,1 & 0,03 & 2 & 3,1 & 0,84 & 13,7 \\
\hline Inalterada & 620,80 & 26,1 & 6,7 & 30 & 0,82 & 0,4 & 0,29 & 0,12 & 2 & 0,49 & $<5$ & $<0,2$ & 0,1 & 3,5 & 0,04 & 1,3 & 2,7 & 0,76 & 16,1 \\
\hline Pre-mineralização & 187,50 & 20 & 2,6 & 30 & 0,2 & 0,11 & 0,11 & 0,07 & 0,9 & 0,15 & $<5$ & $<0,2$ & 0,06 & 1,5 & 0,02 & 0,5 & 1 & 0,28 & 3,1 \\
\hline Pre-mineralização & 513,16 & 15,5 & 2 & 20 & 0,21 & 0,14 & 0,1 & 0,05 & 0,6 & 0,18 & $<5$ & $<0,2$ & 0,04 & 1,1 & 0,01 & 0,5 & 0,8 & 0,23 & 3,8 \\
\hline Pre-mineralização & 492,65 & 49,2 & 3,9 & 30 & 0,61 & 0,25 & 0,17 & 0,09 & 1,4 & 0,25 & $<5$ & $<0,2$ & 0,05 & 2 & 0,03 & 1,1 & 1,6 & 0,44 & 5,7 \\
\hline $1^{\mathrm{a}}$ fase $\mathrm{E}, \mathrm{Min}$ & 48,25 & 20,4 & 8,7 & 30 & 0,49 & 0,5 & 0,26 & 0,13 & 2,3 & 0,49 & $<5$ & $<0,2$ & 0,1 & 4,8 & 0,03 & 1,2 & 3,3 & 0,95 & 14,5 \\
\hline $1^{\mathrm{a}}$ fase $\mathrm{E}, \mathrm{Min}$, & 101,00 & 59,4 & 10,5 & 30 & 0,37 & 0,67 & 0,39 & 0,27 & 2,6 & 1,04 & $<5$ & $<0,2$ & 0,13 & 4,5 & 0,05 & 1,3 & 5,8 & 1,32 & 14,8 \\
\hline Principal E, Min, P,I, & 172,04 & 106,5 & 3,9 & 20 & 0,12 & 0,17 & 0,09 & 0,07 & 1,1 & 0,16 & $<5$ & $<0,2$ & 0,03 & 1,6 & 0,01 & 1,3 & 1 & 0,3 & 0,8 \\
\hline Principal E, Min, P,I, & 181,60 & 10,1 & 1,7 & 50 & 0,05 & 0,13 & 0,11 & 0,05 & 0,7 & 0,13 & $<5$ & $<0,2$ & 0,04 & 1,4 & 0,02 & 0,2 & 0,8 & 0,21 & 0,3 \\
\hline Principal E, Min, P,I, & 183,33 & 4,9 & 15 & 70 & 0,09 & 0,15 & 0,06 & 0,06 & 1,5 & 0,29 & $<5$ & $<0,2$ & 0,05 & 15,6 & 0,02 & 0,4 & 3 & 1,12 & 0,4 \\
\hline Principal E, Min, P,I, & 168,88 & 16 & 6 & 50 & 0,42 & 0,54 & 0,21 & 0,2 & 1,3 & 0,85 & $<5$ & $<0,2$ & 0,11 & 2,9 & 0,02 & 0,2 & 3,4 & 0,72 & 0,9 \\
\hline Principal E, Min, P,I, & 217,24 & 80,8 & 3,5 & 50 & 0,2 & 0,27 & 0,12 & 0,08 & 1,4 & 0,41 & $<5$ & $<0,2$ & 0,07 & 1,6 & 0,02 & 0,2 & 1,5 & 0,39 & 0,5 \\
\hline Principal E, Min, P,I, & 63,36 & 18,1 & 3,1 & 30 & 0,05 & 0,15 & 0,13 & 0,05 & 1,7 & 0,22 & 27 & $<0,2$ & 0,04 & 1,6 & 0,02 & 0,9 & 1,1 & 0,3 & 0,7 \\
\hline Principal E, Min, P,I, & 64,24 & 5,3 & 6,5 & 30 & 0,25 & 0,42 & 0,17 & 0,17 & 2,8 & 0,59 & 5 & $<0,2$ & 0,07 & 2,2 & 0,03 & 0,2 & 2,3 & 0,53 & 0,5 \\
\hline Principal E, Min, M,P,S, & 521,41 & 7,3 & 1,3 & 30 & 0,1 & 0,05 & 0,03 & 0,05 & 4,6 & 0,1 & 22 & $<0,2$ & 0,01 & 0,5 & 0,01 & 0,2 & 0,4 & 0,1 & 0,2 \\
\hline Principal E, Min, M,P,S, & 531,80 & 36,9 & 13,1 & 30 & 0,11 & 0,4 & 0,16 & 0,11 & 2,8 & 0,71 & 14 & $<0,2$ & 0,09 & 2,7 & 0,02 & 0,2 & 2,7 & 0,64 & 0,4 \\
\hline Principal E, Min, M,P,S, & 568,80 & 35,8 & 3,2 & 30 & 0,48 & 0,23 & 0,12 & 0,08 & 2,3 & 0,31 & $<5$ & $<0,2$ & 0,04 & 1,5 & 0,01 & 0,7 & 1,3 & 0,31 & 1,9 \\
\hline $3^{\text {a }}$ fase E,Min, & 169,09 & 899 & 3,8 & 40 & 0,12 & 0,44 & 0,16 & 0,11 & 1 & 0,57 & $<5$ & $<0,2$ & 0,08 & 1,7 & 0,02 & 0,2 & 1,7 & 0,47 & 0,3 \\
\hline $3^{\text {a }}$ fase E,Min, & 255,34 & 15,5 & 3,6 & 40 & 0,22 & 0,36 & 0,23 & 0,13 & 1,2 & 0,36 & $<5$ & 0,2 & 0,09 & 2,3 & 0,04 & 1 & 2 & 0,46 & 2,7 \\
\hline $3^{\mathrm{a}}$ fase E,Min, & 562,05 & 66,6 & 3 & 20 & 0,16 & 0,17 & 0,1 & 0,08 & 1 & 0,29 & $<5$ & $<0,2$ & 0,04 & 1,7 & 0,02 & 0,5 & 1,6 & 0,37 & 1,8 \\
\hline Alt, de Pirita & 273,81 & 57 & 11,9 & 30 & 0,48 & 0,55 & 0,32 & 0,22 & 1,9 & 0,9 & $<5$ & 0,7 & 0,15 & 5,2 & 0,06 & 1,1 & 5,8 & 1,44 & 11,4 \\
\hline Alt, de Pirita & 295,48 & 3530 & 5,6 & 30 & 0,33 & 0,31 & 0,21 & 0,07 & 1,6 & 0,36 & $<5$ & $<0,2$ & 0,07 & 3,1 & 0,02 & 1 & 2,3 & 0,64 & 9,8 \\
\hline Alt, de Pirita & 411,13 & 125,5 & 4,8 & 30 & 0,25 & 0,24 & 0,17 & 0,11 & 1,2 & 0,28 & $<5$ & $<0,2$ & 0,07 & 3,1 & 0,02 & 2,5 & 2,1 & 0,52 & 5,6 \\
\hline Alt, de Pirita & 603,23 & 9,9 & 2,5 & 30 & 0,32 & 0,2 & 0,14 & 0,1 & 0,9 & 0,37 & $<5$ & $<0,2$ & 0,04 & 1,4 & 0,02 & 0,5 & 1,2 & 0,31 & 5,1 \\
\hline Alt, de Pirita & 615,40 & 23,6 & 8,3 & 30 & 0,82 & 0,4 & 0,32 & 0,13 & 1,8 & 0,51 & $<5$ & $<0,2$ & 0,08 & 3,5 & 0,04 & 1,2 & 2,8 & 0,7 & 13,9 \\
\hline
\end{tabular}

Alteração \begin{tabular}{|l|l|l|l|l|l|l|l} 
Amostra & $\mathrm{Sm}$ & $\mathrm{Sn}$ & $\mathrm{Sr}$ & $\mathrm{Ta}$ & $\mathrm{Tb}$ & $\mathrm{Th}$ & $\mathrm{Tm}$ \\
\hline
\end{tabular} \begin{tabular}{l|l|l} 
U & V & W \\
\hline
\end{tabular} \begin{tabular}{|l|l|l|l|l|}
\hline $\mathrm{W}$ & $\mathrm{Y}$ & $\mathrm{Yb}$ & $\mathrm{Zr}$ & $\mathrm{Ag}$ \\
\hline
\end{tabular} 


\begin{tabular}{|c|c|c|c|c|c|c|c|c|c|c|c|c|c|c|c|}
\hline Inalterada & 261,05 & 0,69 & $<1$ & 61,8 & 0,3 & 0,11 & 1,02 & 0,06 & 0,68 & 8 & 96 & 3,7 & 0,44 & 55 & $<0,5$ \\
\hline Inalterada & 338,32 & 0,26 & 5 & 71 & 0,4 & 0,04 & 1,6 & 0,02 & 3,18 & 7 & 151 & 1,4 & 0,11 & 9 & $<0,5$ \\
\hline Inalterada & 498,23 & 0,2 & 1 & 94,5 & 0,4 & 0,03 & 0,37 & $<0,01$ & 0,83 & 5 & 93 & 1,1 & 0,11 & 15 & $<0,5$ \\
\hline Inalterada & 501,52 & 0,51 & 1 & 73,5 & 0,6 & 0,07 & 1,69 & 0,03 & 1,37 & 12 & 223 & 2,3 & 0,24 & 18 & $<0,5$ \\
\hline Inalterada & 620,80 & 0,43 & 1 & 87,2 & 0,4 & 0,08 & 1,25 & 0,05 & 0,85 & 9 & 110 & 2,4 & 0,29 & 13 & $<0,5$ \\
\hline Pre-mineralização & 187,50 & 0,13 & 1 & 62,6 & 0,2 & 0,03 & 0,41 & 0,02 & 0,34 & 17 & 50 & 0,9 & 0,07 & 7 & $<0,5$ \\
\hline Pre-mineralização & 513,16 & $<0,03$ & $<1$ & 62,9 & 0,2 & 0,02 & 0,25 & 0,01 & 0,57 & 6 & 59 & 0,9 & 0,12 & 7 & $<0,5$ \\
\hline Pre-mineralização & 492,65 & 0,23 & 1 & 54,4 & 0,3 & 0,06 & 0,46 & 0,01 & 0,58 & 12 & 119 & 1,4 & 0,1 & 10 & $<0,5$ \\
\hline $1^{\mathrm{a}}$ fase $\mathrm{E}, \mathrm{Min}$, & 48,25 & 0,55 & $<1$ & 53,8 & 0,2 & 0,08 & 1,1 & 0,04 & 0,31 & 8 & 33 & 2,4 & 0,22 & 10 & $<0,5$ \\
\hline $1^{\text {a }}$ fase $\mathrm{E}$, Min, & 101,00 & 1,07 & 1 & 62,4 & 0,3 & 0,13 & 0,93 & 0,04 & 0,39 & 17 & 95 & 4 & 0,25 & 19 & $<0,5$ \\
\hline Principal E, Min, P,I, & 172,04 & 0,12 & 2 & 31,3 & 0,3 & 0,04 & 0,23 & 0,02 & 0,35 & 12 & 545 & 0,9 & 0,08 & 4 & 35 \\
\hline Principal E, Min, P,I, & 181,60 & 0,15 & $<1$ & 22 & 0,3 & 0,02 & 0,12 & 0,02 & 0,47 & 190 & 115 & 1,1 & 0,06 & 3 & $<0,5$ \\
\hline Principal E, Min, P,I, & 183,33 & 0,23 & 1 & 25,6 & 0,3 & 0,03 & 0,17 & 0,02 & 3,36 & 2240 & 134 & 1,2 & 0,08 & 5 & 0,9 \\
\hline Principal E, Min, P,I, & 168,88 & 0,69 & $<1$ & 33 & 0,2 & 0,11 & 0,09 & 0,02 & 4,66 & 100 & 73 & 3,4 & 0,17 & 3 & $<0,5$ \\
\hline Principal E, Min, P,I, & 217,24 & 0,32 & $<1$ & 30,2 & 0,4 & 0,07 & 0,15 & 0,02 & 5,9 & 161 & 146 & 1,8 & 0,13 & 3 & 10,7 \\
\hline Principal E, Min, P,I, & 63,36 & 0,21 & $<1$ & 21,7 & 0,2 & 0,03 & 0,07 & $<0,01$ & 0,62 & 43 & 48 & 1 & 0,12 & 3 & 10,1 \\
\hline Principal E, Min, P,I, & 64,24 & 0,49 & $<1$ & 16,9 & 0,1 & 0,08 & 0,07 & 0,02 & 7,04 & 268 & 66 & 2 & 0,15 & 2 & 4,1 \\
\hline Principal E, Min, M,P,S, & 521,41 & 0,03 & $<1$ & 22,3 & 0,4 & 0,02 & $<0,05$ & 0,01 & 1,78 & 53 & 153 & $<0,5$ & 0,04 & 2 & 14,8 \\
\hline Principal E, Min, M,P,S, & 531,80 & 0,51 & 9 & 13 & 0,3 & 0,11 & $<0,05$ & $<0,01$ & 2,44 & 79 & 150 & 2,5 & 0,09 & 3 & 5,6 \\
\hline Principal E, Min, M,P,S, & 568,80 & 0,11 & 1 & 30,4 & 0,2 & 0,05 & 0,19 & $<0,01$ & 3,46 & 212 & 108 & 1,3 & 0,11 & 4 & 0,7 \\
\hline $3^{\text {a }}$ fase E,Min, & 169,09 & 0,38 & $<1$ & 46,1 & 0,2 & 0,09 & $<0,05$ & 0,02 & 1,98 & 37 & 88 & 2,7 & 0,15 & 2 & 0,7 \\
\hline $3^{\text {a }}$ fase E,Min, & 255,34 & 0,26 & 1 & 47,1 & 0,3 & 0,07 & 0,42 & 0,04 & 1,5 & 76 & 85 & 2,8 & 0,17 & 27 & $<0,5$ \\
\hline $3^{\mathrm{a}}$ fase E,Min, & 562,05 & 0,21 & $<1$ & 51,7 & 0,3 & 0,07 & 0,28 & 0,01 & 0,39 & $<5$ & 101 & 1,5 & 0,07 & 7 & $<0,5$ \\
\hline Alt, de Pirita & 273,81 & 1,03 & 1 & 43,1 & 0,4 & 0,13 & 0,84 & 0,04 & 0,86 & 16 & 166 & 3,6 & 0,36 & 48 & 1,3 \\
\hline Alt, de Pirita & 295,48 & 0,33 & $<1$ & 132,5 & 0,3 & 0,06 & 0,53 & 0,03 & 0,91 & 22 & 80 & 1,8 & 0,28 & 13 & $<0,5$ \\
\hline Alt, de Pirita & 411,13 & 0,27 & 1 & 55,6 & 0,6 & 0,05 & 0,49 & 0,02 & 0,34 & 13 & 234 & 1,5 & 0,15 & 12 & $<0,5$ \\
\hline Alt, de Pirita & 603,23 & 0,19 & $<1$ & 61,5 & 0,2 & 0,04 & 0,38 & 0,02 & 0,39 & $<5$ & 90 & 1,2 & 0,13 & 6 & $<0,5$ \\
\hline Alt, de Pirita & 615,40 & 0,56 & 4 & 89,8 & 0,3 & 0,08 & 0,9 & 0,03 & 0,49 & 9 & 100 & 2,6 & 0,22 & 13 & $<0,5$ \\
\hline
\end{tabular}




\begin{tabular}{|c|c|c|c|c|c|c|c|c|c|c|}
\hline Alteração & Amostra & $\mathbf{C d}$ & Co & $\mathbf{C u}$ & $\mathbf{L i}$ & Mo & $\mathbf{N i}$ & $\mathbf{P b}$ & Sc & $\operatorname{Zn}(\%)$ \\
\hline Inalterada & 261,05 & 0,6 & 12 & 4 & $<10$ & $<1$ & 1 & 104 & 1 & 0,0272 \\
\hline Inalterada & 338,32 & $<0,5$ & 124 & 10 & $<10$ & $<1$ & $<1$ & 23 & $<1$ & 0,0021 \\
\hline Inalterada & 498,23 & 0,9 & 15 & 3 & $<10$ & $<1$ & 1 & 17 & $<1$ & 0,0138 \\
\hline Inalterada & 501,52 & 0,9 & 46 & $<1$ & 10 & $<1$ & 1 & 66 & 1 & 0,044 \\
\hline Inalterada & 620,80 & $<0,5$ & 16 & 1 & 10 & $<1$ & 1 & 6 & 1 & 0,0045 \\
\hline Pre-mineralização & 187,50 & 2,5 & 10 & 3 & $<10$ & $<1$ & 3 & 156 & 1 & 0,0988 \\
\hline Pre-mineralização & 513,16 & 1,9 & 8 & 2 & $<10$ & $<1$ & $<1$ & 63 & $<1$ & 0,0363 \\
\hline Pre-mineralização & 492,65 & 3,1 & 18 & 24 & 10 & $<1$ & 3 & 248 & 1 & 0,0579 \\
\hline $1^{a}$ fase $E$, Min, & 48,25 & 9,5 & 5 & 4 & $<10$ & $<1$ & 4 & 461 & 1 & 0,189 \\
\hline $1^{\mathrm{a}}$ fase $\mathrm{E}, \mathrm{Min}$, & 101,00 & 3,7 & 13 & 2 & 10 & $<1$ & 8 & 302 & 1 & 0,128 \\
\hline Principal E, Min, P,I, & 172,04 & 309 & 76 & 455 & $<10$ & $<1$ & 9 & 2300 & $<1$ & 20,7 \\
\hline Principal E, Min, P,I, & 181,60 & 14,8 & 18 & 8 & $<10$ & $<1$ & 18 & 895 & $<1$ & 0,369 \\
\hline Principal E, Min, P,I, & 183,33 & 43,2 & 28 & 6 & $<10$ & 1 & 24 & 2200 & $<1$ & 0,455 \\
\hline Principal E, Min, P,I, & 168,88 & 330 & 26 & 5 & $<10$ & 1 & 10 & 4680 & $<1$ & 1,49 \\
\hline Principal E, Min, P,I, & 217,24 & 262 & 30 & 41 & $<10$ & 1 & 7 & 3020 & $<1$ & 4,91 \\
\hline Principal E, Min, P,I, & 63,36 & 135 & 2 & 30 & $<10$ & $<1$ & 10 & 3740 & $<1$ & 33,91 \\
\hline Principal E, Min, P,I, & 64,24 & 61,3 & 34 & 39 & $<10$ & 2 & 30 & 4090 & $<1$ & 2,12 \\
\hline Principal E, Min, M,P,S, & 521,41 & 76,8 & 29 & 65 & $<10$ & 1 & 10 & 1365 & $<1$ & 39,81 \\
\hline Principal E, Min, M,P,S, & 531,80 & 265 & 21 & 43 & $<10$ & 2 & 19 & 1640 & $<1$ & 37,45 \\
\hline Principal E, Min, M,P,S, & 568,80 & 207 & 38 & 16 & $<10$ & 3 & 21 & 1880 & $<1$ & 4,48 \\
\hline $3^{\text {a }}$ fase E,Min, & 169,09 & 339 & 38 & 4 & $<10$ & $<1$ & 13 & 4490 & $<1$ & 1,365 \\
\hline $3^{\text {a }}$ fase E,Min, & 255,34 & 12,4 & 24 & 6 & $<10$ & $<1$ & 9 & 597 & 1 & 0,52 \\
\hline $3^{\text {a }}$ fase E,Min, & 562,05 & 186,5 & 52 & 17 & $<10$ & $<1$ & 15 & 3860 & $<1$ & 1,475 \\
\hline Alt, de Pirita & 273,81 & 1,6 & 33 & 57 & $<10$ & $<1$ & 19 & 99 & 1 & 0,0307 \\
\hline Alt, de Pirita & 295,48 & 1,5 & 13 & 28 & $<10$ & $<1$ & 3 & 49 & 1 & 0,0305 \\
\hline Alt, de Pirita & 411,13 & 5,4 & 32 & 90 & $<10$ & $<1$ & $<1$ & 31 & 1 & 0,0285 \\
\hline Alt, de Pirita & 603,23 & 1,3 & 15 & 4 & $<10$ & $<1$ & $<1$ & 120 & $<1$ & 0,019 \\
\hline Alt, de Pirita & 615,40 & 0,6 & 16 & 1 & 10 & $<1$ & 2 & 32 & 1 & 0,0166 \\
\hline
\end{tabular}




\begin{tabular}{|c|c|c|c|c|c|c|c|c|c|c|c|c|c|}
\hline Alteração & Amostra & As & $\mathbf{B i}$ & $\mathbf{H g}$ & In & $\mathbf{R e}$ & Sb & Sc & Se & Te & $\mathbf{T l}$ & $\mathrm{S}(\%)$ & $\mathrm{C}(\%)$ \\
\hline Inalterada & 261,05 & 0,6 & 0,03 & 0,228 & 0,005 & $<0,001$ & 0,07 & 1,4 & 0,5 & 0,01 & 0,02 & 0,01 & 12 \\
\hline Inalterada & 338,32 & 0,4 & 0,13 & 0,188 & $<0,005$ & 0,001 & $<0,05$ & 0,5 & 0,2 & $<0,01$ & 0,02 & $<0,01$ & 12,9 \\
\hline Inalterada & 498,23 & 0,3 & 0,01 & 0,175 & $<0,005$ & $<0,001$ & $<0,05$ & 0,4 & 0,2 & 0,01 & 0,02 & $<0,01$ & 12,5 \\
\hline Inalterada & 501,52 & 0,3 & 0,09 & 0,28 & 0,005 & 0,001 & $<0,05$ & 1,2 & 0,3 & $<0,01$ & 0,04 & 0,01 & 12,2 \\
\hline Inalterada & 620,80 & $<0,1$ & 0,05 & 0,118 & $<0,005$ & $<0,001$ & $<0,05$ & 0,9 & $<0,2$ & $<0,01$ & 0,03 & $<0,01$ & 12,3 \\
\hline Pre-mineralização & 187,50 & 2,1 & 0,01 & 0,123 & $<0,005$ & 0,001 & 0,05 & 0,6 & 0,3 & 0,01 & $<0,02$ & 0,01 & 12,9 \\
\hline Pre-mineralização & 513,16 & 0,3 & 0,02 & 0,101 & $<0,005$ & 0,001 & $<0,05$ & 0,4 & 0,3 & $<0,01$ & $<0,02$ & $<0,01$ & 12,6 \\
\hline Pre-mineralização & 492,65 & 2,4 & 0,04 & 0,429 & 0,009 & 0,002 & 0,1 & 0,7 & $<0,2$ & 0,02 & 0,04 & 0,11 & 9,85 \\
\hline $1^{\mathrm{a}}$ fase $\mathrm{E}, \mathrm{Min}$, & 48,25 & 2,4 & 0,01 & 0,063 & 0,006 & $<0,001$ & 0,09 & 1,3 & 0,3 & 0,02 & 0,02 & $<0,01$ & 12,1 \\
\hline $1^{\mathrm{a}}$ fase $\mathrm{E}, \mathrm{Min}$, & 101,00 & 4,9 & 0,04 & 0,144 & 0,007 & $<0,001$ & 0,08 & 1,3 & 0,4 & 0,01 & 0,02 & 0,01 & 11,3 \\
\hline Principal E, Min, P,I, & 172,04 & 18,2 & 0,12 & 3,32 & 0,024 & 0,003 & 0,24 & 0,6 & 12,7 & 0,02 & 0,05 & $<0,01$ & 7 \\
\hline Principal E, Min, P,I, & 181,60 & 3,5 & 0,02 & 0,177 & $<0,005$ & 0,001 & 0,58 & 0,2 & 0,4 & 0,07 & 0,03 & 0,01 & 13 \\
\hline Principal E, Min, P,I, & 183,33 & 18,8 & 0,11 & 0,285 & $<0,005$ & 0,001 & 9,95 & 0,2 & 0,6 & 0,37 & $<0,02$ & $<0,01$ & 7,36 \\
\hline Principal E, Min, P,I, & 168,88 & 52,5 & 0,03 & 0,994 & 0,63 & $<0,001$ & 12,3 & 0,3 & 1,1 & 0,01 & $<0,02$ & 0,01 & 9,37 \\
\hline Principal E, Min, P,I, & 217,24 & 53,9 & 0,06 & 0,478 & 0,218 & $<0,001$ & 8,34 & 1 & 3,4 & 0,01 & 0,02 & $<0,01$ & 6,08 \\
\hline Principal E, Min, P,I, & 63,36 & 110 & $<0,01$ & 0,557 & 0,165 & $<0,001$ & 4,33 & 0,4 & 18 & 0,02 & $<0,02$ & 0,01 & 4,42 \\
\hline Principal E, Min, P,I, & 64,24 & 54,8 & 0,05 & 0,108 & 0,458 & $<0,001$ & 42,4 & 0,7 & 1,1 & $<0,01$ & $<0,02$ & 0,01 & 3,28 \\
\hline Principal E, Min, M,P,S, & 521,41 & 26,2 & 0,04 & 0,289 & 0,254 & $<0,001$ & 1,91 & 0,3 & 23,5 & 0,02 & $<0,02$ & 0,01 & 2,63 \\
\hline Principal E, Min, M,P,S, & 531,80 & 74,2 & 0,02 & 0,222 & 0,6 & $<0,001$ & 4,35 & 0,4 & 21,3 & 0,02 & $<0,02$ & $<0,01$ & 2,14 \\
\hline Principal E, Min, M,P,S, & 568,80 & 16,1 & 0,04 & 0,106 & 0,813 & 0,001 & 10,25 & 1 & 2,7 & 0,01 & $<0,02$ & $<0,01$ & 4,16 \\
\hline $3^{\text {a }}$ fase E,Min, & 169,09 & 23 & 0,01 & 0,336 & 0,142 & $<0,001$ & 5,56 & 0,2 & 1 & 0,01 & $<0,02$ & 0,01 & 11,7 \\
\hline $3^{\mathrm{a}}$ fase E,Min, & 255,34 & 2,8 & 0,09 & 0,172 & 0,009 & 0,001 & 0,21 & 0,7 & 0,5 & 0,04 & $<0,02$ & $<0,01$ & 12,3 \\
\hline $3^{\mathrm{a}}$ fase E,Min, & 562,05 & 2,8 & 0,02 & 0,138 & 0,035 & $<0,001$ & 0,21 & 0,4 & 1,2 & 0,01 & $<0,02$ & $<0,01$ & 12,9 \\
\hline Alt, de Pirita & 273,81 & 33,5 & 0,02 & 0,21 & 0,013 & 0,002 & 0,61 & 1 & 0,5 & 0,02 & 0,11 & 7,35 & 9,42 \\
\hline Alt, de Pirita & 295,48 & 2,8 & 0,03 & 0,111 & $<0,005$ & 0,001 & 0,17 & 0,8 & $<0,2$ & 0,01 & 0,02 & 2,75 & 11,2 \\
\hline Alt, de Pirita & 411,13 & 1,1 & 0,01 & 0,303 & 0,013 & 0,001 & $<0,05$ & 0,6 & 0,4 & 0,02 & 0,03 & 0,01 & 8,27 \\
\hline Alt, de Pirita & 603,23 & 6,5 & 0,01 & 0,146 & 0,009 & $<0,001$ & 0,74 & 0,4 & 0,2 & $<0,01$ & 0,04 & 3,88 & 11,2 \\
\hline Alt, de Pirita & 615,40 & 1 & 0,01 & 0,153 & 0,01 & $<0,001$ & $<0,05$ & 0,9 & 0,4 & 0,01 & 0,03 & 0,01 & 12,4 \\
\hline
\end{tabular}

